THE ROLE OF INTERFACIAL PHENOMENA IN LEAKAGE FROM GEOLOGIC CARBON SEQUESTRATION SITES

\author{
A Dissertation \\ Presented to \\ the faculty of the School of Engineering and Applied Science \\ University of Virginia \\ in partial fulfillment \\ of the requirements for the degree \\ Doctor of Philosophy \\ by
}

Shibo Wang

May

2013 
APPROVAL SHEET

The dissertation

is submitted in partial fulfillment of the requirements

for the degree of

Doctor of Philosophy

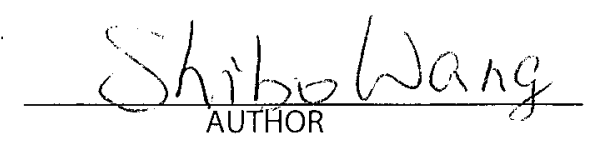

The dissertation has been read and approved by the examining committee:

\begin{tabular}{c} 
Andres Clarens \\
\hline $\begin{array}{c}\text { Advisor } \\
\text { James Smith }\end{array}$ \\
\hline Teresa Culver \\
\hline Roseanne Ford
\end{tabular}

Catherine Peters

Accepted for the School of Engineering and Applied Science:

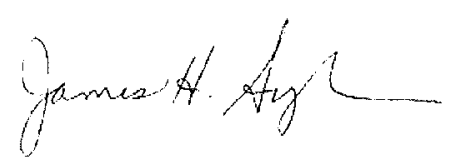

Dean, School of Engineering and Applied Science

May

2013 


\title{
THE ROLE OF INTERFACIAL PHENOMENA IN LEAKAGE FROM GEOLOGIC CARBON SEQUESTRATION SITES
}

\author{
by \\ Shibo Wang \\ A dissertation submitted in partial fulfillment \\ of the requirements for the degree of \\ Doctor of Philosophy \\ Civil and Environmental Engineering \\ at The University of Virginia
}

Approved as to style and content by Doctoral Committee:

James Smith, Chair, Professor of CEE, University of Virginia

Andres Clarens, Adviser, Assistant Professor of CEE, University of Virginia

Teresa Culver, Associate Professor of CEE, University of Virginia

Roseanne Ford, Professor of CHE, University of Virginia

Catherine Peters, Professor of CEE, Princeton University 
(C) Shibo Wang 2013 
To my beloved parents, grandparents and all family members In memory of my grandmother 


\section{Acknowledgements}

This dissertation will not exist without the huge supports from my parents and my beloved family members. I feel fortunate and blessed to be born in a family full of care and love. I thank them for not only raising me up, teaching me to value hard work, to become a man of good deeds and of integrity, but also providing me with the freedom to explore the truth. They are the strength which made me through years of study overseas.

For the past two years, Yiwen has been as supportive as one could imagine. Just as her English name Grace, she has been so graceful and cheerful, whose company and friendship has made the hectic research days much happier.

I would like to express my sincere gratitude to my mentors. As a great adviser, a phenomenal researcher, a well-respected teacher and a close friend, Dr. Andres Clarens has always supported and motivated me to discover the unknowns in science, pursue eminence and conduct high quality research. I feel very privileged to have worked with such a brilliant guide and friend. Dr. James Smith and Dr. Teresa Culver have showed great interest and kindness to insightfully guide my study. Dr. Roseanne Ford and Dr. Catherine Peters have offered great wisdom and perspectives to both my research and career development.

All of the wok presented herein was performed with the kind cooperation and collaboration with my lab mates in the VESTLab: Mark Santana, Brian Weaver, Alec Gosse, Zhiyuan Tao, Bo Liang, Xiaowei Liu and Eleazer Resurreccion. I am hugely grateful to their company, friendship and support. The VESTLab people have made my life at UVa like home and made my days full of sunshine and happiness.

I feel lucky to be the mentor of the intelligent undergraduate researchers: Brian Tison, 
Karla Sharrer, Jasmine Copeland, Brandon Lee, Ian Edwards, Sara Persily, Simon Kobayashi, Lauren Bolton, Christina Perry and Jack St. Marie, from whom I have had outstanding assistance in research and have learned how to be a wise adviser and a good team leader. Their passion, their love of learning have deeply impressed and motivated me for scientific exploration.

I want to thank the faculty and staff at the University of Virginia, especially those in the Department of Civil and Environmental Engineering: Dr. Brian Smith, Dr. Wu-Seng Lung, Dr. Lisa Colosi, James Danberg, Pat Vance, Kimberly Olsen and Peggy Gibson. They have made my life much easier and there would not be any success in my work without their help.

My collaborators including Dr. Amir Younan, Dr. Jeffery Fitts, Dr. Hongbo Shao and Dr. Dedong Li have offered insightful advices on my research. They are all excellent researchers and fabulous people in life.

My awesome Chinese and American friends, too many to mention explicitly, have enlightened my life in the U.S. I want to thank everyone for the wonderful time we have shared together.

The research described here was supported in part by the University of Virginia School of Engineering and Applied Science through faculty start-up funds, and the National Science Foundation (Grant \# CBET-1134397).

You all have filled my life with wonderful dreams. Thank you! 


\title{
List of Symbols
}

\author{
$X_{C S} \quad \mathrm{CO}_{2}$ solubility under a specific temperature and pressure condition \\ $P \quad$ Pressure (MPa) \\ $T \quad$ Temperature $\left({ }^{\circ} \mathrm{C}\right)$ \\ $t \quad$ Time $(\mathrm{s})$ \\ $S \quad$ Salinity, mole fraction of $\mathrm{NaCl}(\mathrm{M})$ \\ $g \quad$ Gravitational acceleration $\left(\mathrm{m} / \mathrm{s}^{2}\right)$ \\ $u \quad$ Rise velocity of $\mathrm{CO}_{2}(\mathrm{~m} / \mathrm{s})$ \\ $d_{\mathrm{CO}_{2}} \quad$ Diameter of $\mathrm{CO}_{2}$ bubble (m) \\ $\rho_{\text {matrix }} \quad$ Density of brine $\left(\mathrm{kg} / \mathrm{m}^{3}\right)$ \\ $\rho_{\mathrm{CO}_{2}} \quad$ Density of $\mathrm{CO}_{2}\left(\mathrm{~kg} / \mathrm{m}^{3}\right)$ \\ $\mu_{\text {matrix }} \quad$ Dynamic viscosity of matrix fluid (Pa.s) \\ $\mu_{\mathrm{CO}_{2}} \quad$ Dynamic viscosity of $\mathrm{CO}_{2}(\mathrm{~Pa} \cdot \mathrm{s})$ \\ $V \quad$ Volume of $\mathrm{CO}_{2}$ bubble $\left(\mathrm{m}^{3}\right)$ \\ $\frac{D}{D t} \quad$ Material derivative of time \\ $y \quad$ Depth (m) \\ A Correction factor in Ergun's equation \\ $R_{\mathrm{CO}_{2}} \quad$ Equivalent radius of $\mathrm{CO}_{2}(\mathrm{~m})$ \\ $\sum F \quad$ The sum of forces acting on $\mathrm{CO}_{2}(\mathrm{~N})$ \\ $F_{b} \quad$ Buoyancy force $(\mathrm{N})$ \\ $F_{s t} \quad$ Interfacial tension force $(\mathrm{N})$ \\ $F_{d} \quad$ Drag force $(\mathrm{N})$
}




\begin{tabular}{|c|c|}
\hline$\phi$ & Effective porosity of porous media (\%) \\
\hline $\operatorname{Re}$ & Reynolds number \\
\hline $\mathrm{Ca}$ & Capillary number \\
\hline Bo & Bond number \\
\hline$\theta$ & Contact angle $\left({ }^{\circ}\right)$ \\
\hline$\sigma$ & Interfacial tension of $\mathrm{CO}_{2}$-brine $(\mathrm{N} / \mathrm{m})$ \\
\hline$R_{p}$ & Pore throat $(\mathrm{m})$ \\
\hline$d_{p}$ & Grain diameter of porous media $(\mathrm{m})$ \\
\hline$C_{i}$ & The aqueous concentrations of the volatile species (mole fractions, \%) \\
\hline$H_{i}$ & The Henry's law coefficients for each species $i(\mathrm{MPa})$ \\
\hline$P_{y}$ & The hydrostatic pressure at depth $y(\mathrm{MPa})$ \\
\hline$P_{s t}$ & The interfacial tension pressure $(\mathrm{MPa})$ \\
\hline$\gamma$ & The viscosity coefficient used in Stokes Law \\
\hline$A_{i j}$ & Hamaker constant $(\mathrm{J})$ \\
\hline$f$ & Thickness of water film (m) \\
\hline$\Pi_{v d W}$ & Disjoining pressure of water film from van der Waals interactions $(\mathrm{Pa})$ \\
\hline$k_{B}$ & Boltzmann constant $\left(\mathrm{J} \cdot \mathrm{K}^{-1}\right)$ \\
\hline$h$ & Planck constant $(\mathrm{J} \cdot \mathrm{s})$ \\
\hline$v_{e}$ & Primary electronic absorption frequency in the ultraviolet region $\left(\mathrm{s}^{-1}\right)$ \\
\hline$\varepsilon_{i}$ & Dielectric constant \\
\hline$n_{i}$ & Refractive index \\
\hline
\end{tabular}




\section{ABSTRACT}

Geologic carbon sequestration (GCS) in deep saline aquifers is a scalable approach for keeping large volumes of anthropogenic carbon dioxide $\left(\mathrm{CO}_{2}\right)$ out of the atmosphere. In GCS, the flue gas from power plants and other point sources is captured, separated, compressed, transported and injected into porous geologic formations at depths greater than one kilometer below the earth surface. In these formations, $\mathrm{CO}_{2}$ exists in the liquid or supercritical phase because of large hydrostatic pressures and geothermal temperatures. Buoyancy driven rise of the $\mathrm{CO}_{2}$ is prevented by overlying impermeable "caprock" layers and also by secondary processes including capillary trapping and mineral precipitation of $\mathrm{CO}_{2}$. Despite its promise, a number of concerns exist about the safety and economic viability of GCS and most of these concerns are related to leakage of $\mathrm{CO}_{2}$ from target formations. $\mathrm{CO}_{2}$ injected into the subsurface could leak via a number of pathways including porous media, abandoned well bores, fractures in the bounding formation, and geologic faults. The processes that lead to leakage are difficult to study in the field because the heterogeneity of the subsurface, complex phase behavior, trapping and transport at multiple scales, and chemical reactions make cause-and-effect relationships difficult to establish.

The overarching goal of this work is to develop a fundamental understanding of how interfacial properties at the $\mathrm{CO}_{2}$-brine-mineral interfaces could impact buoyancy driven flow through porous media and thus control carbon leakage. Both experimental characterization of key parameters (i.e., effective viscosity of multiphase mixtures and contact angle at the $\mathrm{CO}_{2^{-}}$ brine-mineral interface) and theoretical modeling (i.e., expansions of Darcy's law and the Ergun equation) are used to achieve this objective. Several key hypotheses were tested: 1 . multiphase 
binary mixtures of $\mathrm{CO}_{2}$ in brine will result in non-Newtonian rheological behavior; 2 . the brine chemistry will have an important effect on controlling the interfacial wetting of minerals when contacted with $\mathrm{CO}_{2} ; 3$. important differences will exist in wettability for minerals that tend to exist on the pore surface rather than in the bulk of the porous media; and 4. at larger scales, these processes will lead to preferential flow paths and hysteresis that will be important to understand when calculating leakage rates.

The results suggest that once leakage ensues, the presence of $\mathrm{CO}_{2(\mathrm{~g})}$ bubbles imparts complex linear viscoelastic, time dependent, and thixotropic behavior on the $\mathrm{CO}_{2}$-brine mixture. The apparent viscosity of these mixtures was found to be up to five times higher than brine alone though at higher temperatures and/or ionic strength, the effect was less pronounced. At the mineral interface, several representative solids including quartz, microcline, calcite, kaolinite, phlogopite, illite and dolomite were all were found to be water wetting $\left(\theta<30^{\circ}\right)$ with subtle but important differences in contact angles. Temperature and pressure conditions did not produce discernible trends common to all surfaces, but brine $\mathrm{pH}$ and ionic strength, was a better predictor of interfacial behavior. Wettability is impacted by the $\mathrm{pH}$ at the point of zero charge of the solid. Under non-equilibrium conditions, hysteretic effects were observed when $\mathrm{CO}_{2}$ was dissolving into the bulk fluid and this effect varied between minerals. Strong adhesion of $\mathrm{CO}_{2}$ bubbles was observed on certain mineral surfaces and this important behavior was driven in part by surface morphology, the short-range van der Waals interactions and the chemical composition of the hydrated surface. The experimental parameters measured here were incorporated into an improved model of buoyancy-driven transport through porous media in brine and the outputs of this model suggest that dissolution of $\mathrm{CO}_{2}$ in brines and residual saturation trapping could act to suppress vertical transport. 


\section{Table of Contents}

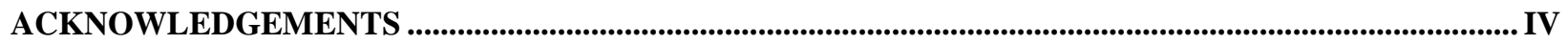

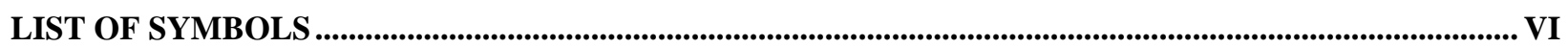

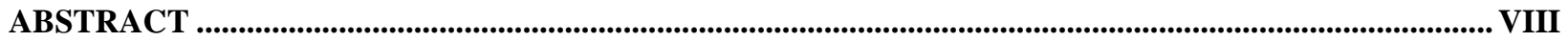

INTRODUCTION ..........................................................................................................................................................1

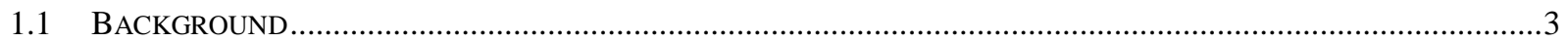

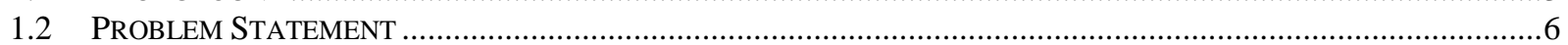

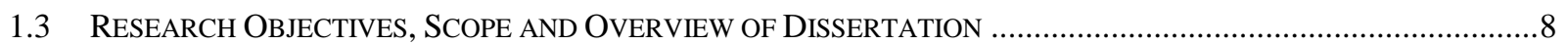

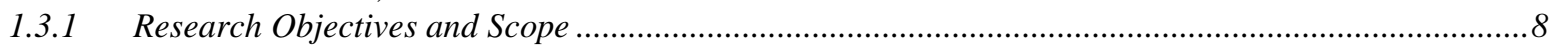

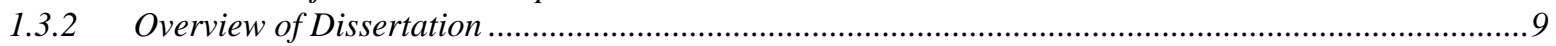

LITERATURE REVIEW ..............................................................................................................................................12

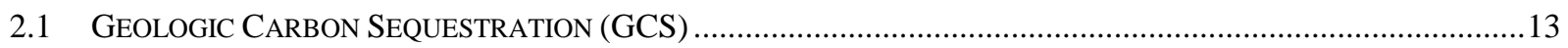

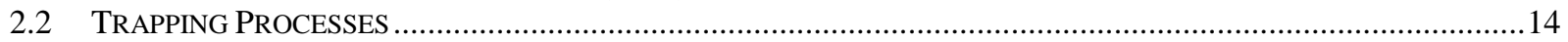

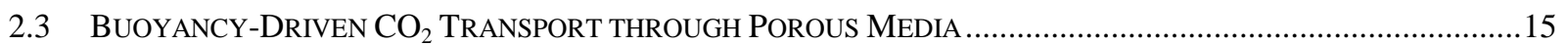

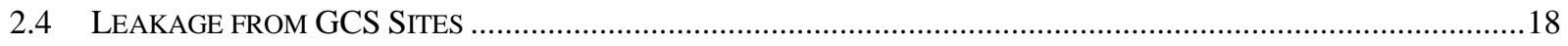

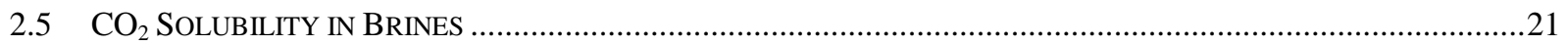

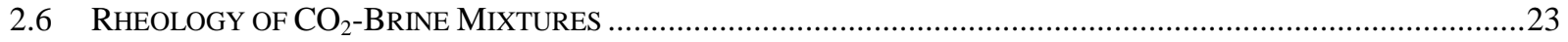

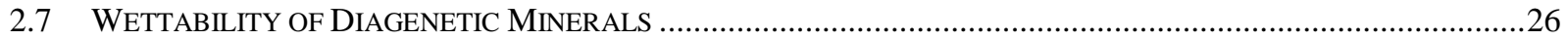

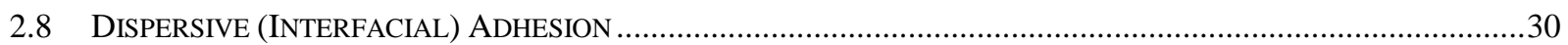

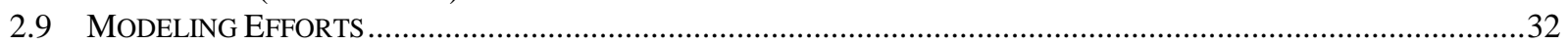

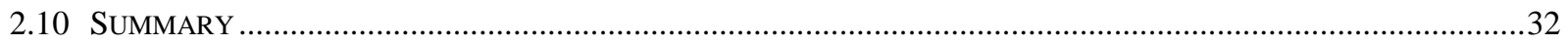

RHEOLOGY OF CO $\mathrm{CO}_{2}$-BRINE MIXTURES..........................................................................................................35

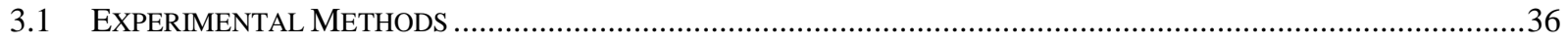

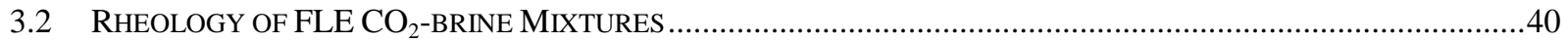

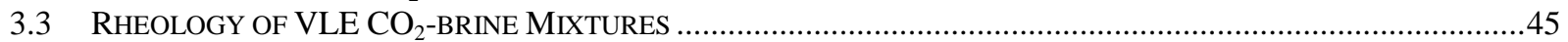

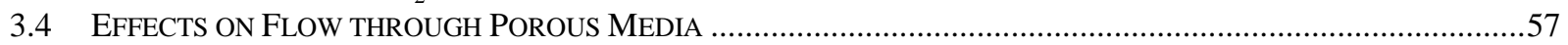

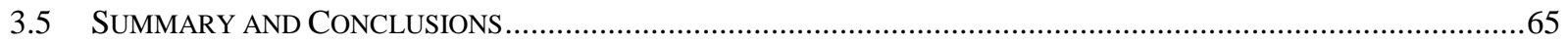

WETTABILITY PHENOMENA AT THE $\mathrm{CO}_{2}$-BRINE-MINERAL INTERFACE ........................................67

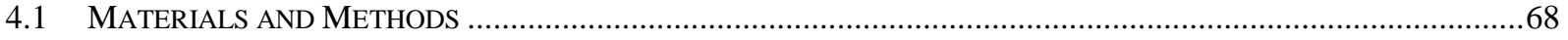

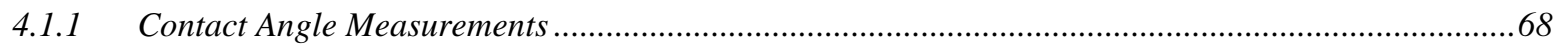

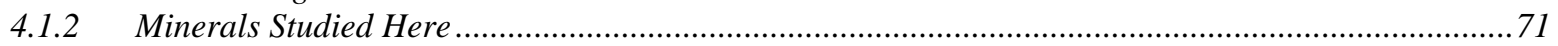

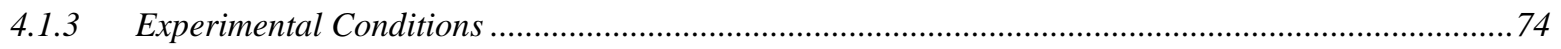

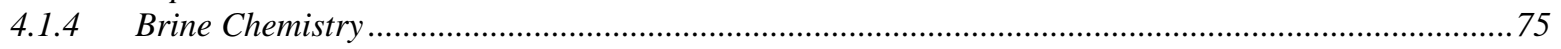

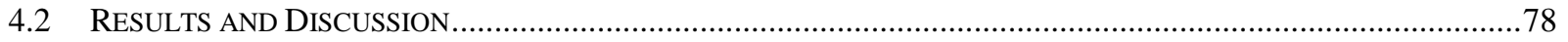

4.2.1 Effect of Pressure, Temperature, Ionic Strength, and pH on Contact Angles at Equilibrium ............. 78

4.2.2 Salt Deposition and Surface Characterization ..................................................................................82

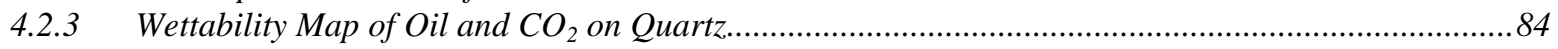

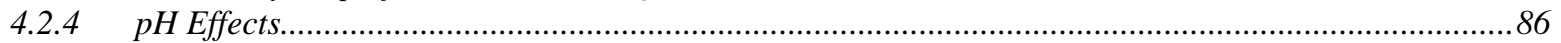

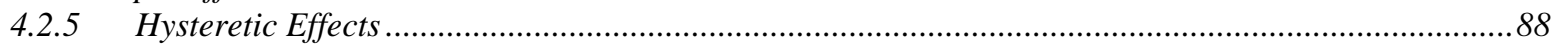

4.2.6 Phase Change inside $\mathrm{CO}_{2}$ Droplets and Its Effects ...............................................................92

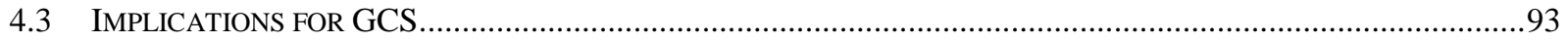

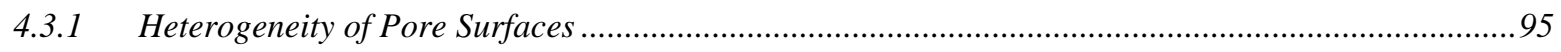

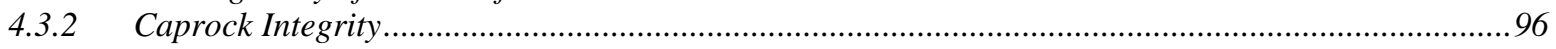

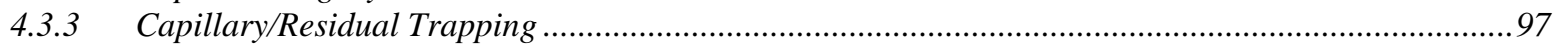

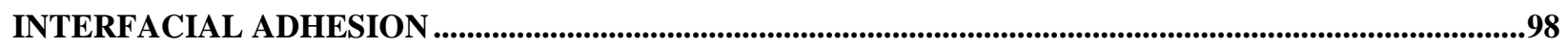

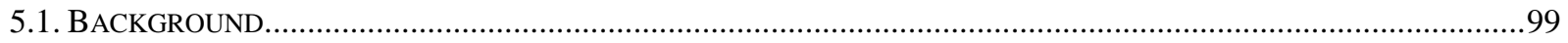

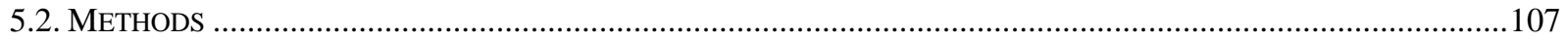




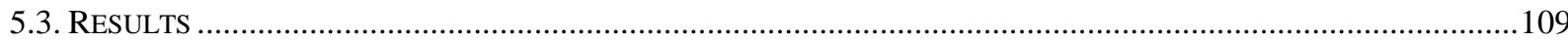

MODELING VERTICAL MIGRATION OF $\mathrm{CO}_{2}$ THROUGH POROUS MEDIA........................................121

6.1 IMPROVED ForCE B ALANCE INTEGRATING EXPERIMENTAL DATA OBTAINED HERE ..................................122

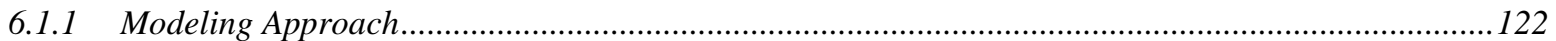

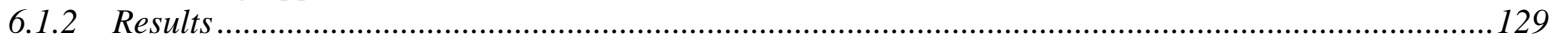

6.2 GANGLION MODEL SIMULATION USING RESERVOIR DATA ............................................................... 135

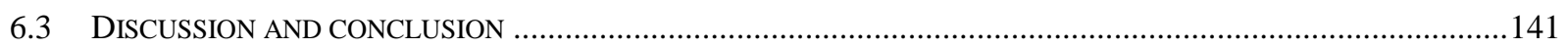

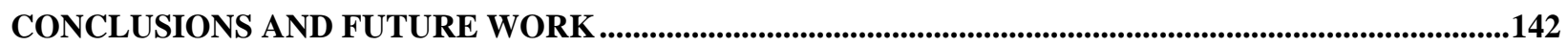

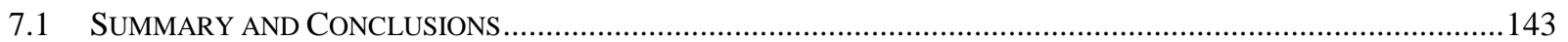

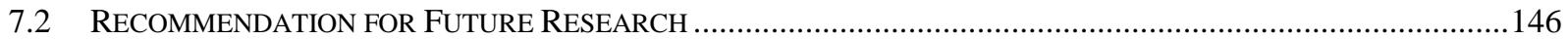

7.2.1 Identify $\mathrm{CO}_{2}$-Adhesive Minerals .............................................................................................146

7.2.2 Explore the Mechanism of Adhesion with Molecular Dynamics Simulation .....................................147

7.2.3 Improve Modeling Framework of $\mathrm{CO}_{2}$ Transport ...................................................................... 148

7.2.4 Model Validation Using Small/Intermediate-Scale Columns ......................................................148

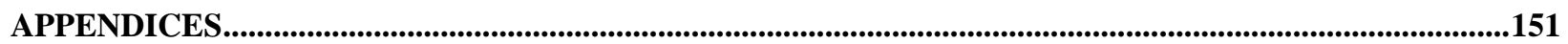

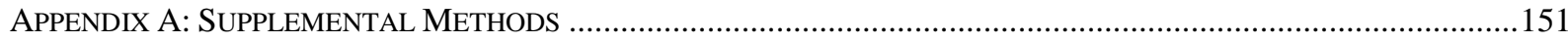

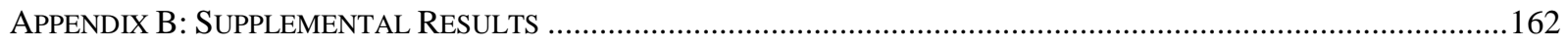

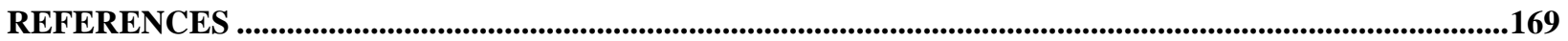




\section{Chapter 1}

\section{Introduction}

Human activity is affecting the climate of the earth on a wide scale [NRC, 2010]. Massive production and emissions of anthropogenic greenhouse gases, largely $\mathrm{CO}_{2}$ generated from industrial or energy-related sources by the burning of fossil fuels, contribute to radiative forcing of the sun's rays, heating the earth's atmosphere. The UN Intergovernmental Panel on Climate Change (IPCC) has concluded that to limit global-average temperature rise to less than $2.4^{\circ} \mathrm{C}$, global $\mathrm{CO}_{2}$ emissions will need to be reduced by 2050 to at least $50 \%$ relative to 2000 levels [IPCC, 2005]. Intensive scientific research has been conducted to mitigate and potentially eliminate the adverse effects associated with global warming. Among the proposed global climate change mitigation strategies, geological carbon sequestration (GCS) is one of the most promising because it is proven to be deployed at a scale that is large enough to make meaningful reductions in global emissions.

In $\mathrm{GCS}, \mathrm{CO}_{2}$ from fossil fuel combustion emissions is screened, compressed, and transported to an injection site where it is stored at depths below $1 \mathrm{~km}$. At these depths, the hydrostatic pressures and geothermal temperatures are large enough to cause the $\mathrm{CO}_{2}$ to exist in the liquid or supercritical phase [Chen and Zhang, 2010; Yang and Gu, 2006; Zhang et al., 2009]. The In- 
ternational Energy Agency projects that GCS should account for as much as $19 \%$ of the needed emissions reductions, second only to end-use efficiency increases in terms of total emissions avoided [Bhattacharya, 2011]. For the U.S., a similar analysis by the Electric Power Research Institute estimates that GCS could account for one third of the needed reductions, surpassing even end-use efficiency [Bhattacharya, 2011]. The capacity for GCS is enormous making it unique among $\mathrm{CO}_{2}$ storage technologies and the formations in which $\mathrm{CO}_{2}$ could be stored are geographically distributed across the U.S. $16,000 \mathrm{~km}^{2}$ of rock formations have been mapped in the U.S. that could be used to sequestrate 500 years' worth of U.S. carbon dioxide emissions based on current emissions rates [Live Science, 2009]. As of middle 2011, there have been 74 large-scale integrated carbon capture and sequestration (CCS) projects globally with eight of them in current operation [Bhattacharya, 2011].

The biggest risk associated with wide-scale deployment of GCS is that $\mathrm{CO}_{2}$ could leak from target formations up to the ground. This would undermine the technology and represent a major contributor to climate change given how much energy would be wasted putting the $\mathrm{CO}_{2}$ in the ground only to have it re-enter the atmosphere. Even though $\mathrm{CO}_{2}$ would exist as a liquid or supercritical fluid in the subsurface, it would still be subject to buoyant forces. If structural trapping, e.g., caprocks fail, then there would be a means for the $\mathrm{CO}_{2}$ to eventually migrate to the surface. The goal of this dissertation is to explore the interfacial properties that will control buoyancy-driven $\mathrm{CO}_{2}$ transport. 


\subsection{Background}

GCS is most often discussed in the context of using sedimentary basins, primarily deep saline aquifers as target repositories since these are abundant and have little economic value [Chen et al., 2003; Eccles et al., 2009]. Other target formations include depleted oil and gas fields and deep unmineable coal seams. At these depths, hydrostatic pressures and geothermal temperatures are large and the $\mathrm{CO}_{2}$ exists in the liquid or supercritical phase where it would intermingle with the connate brines [Chen and Zhang, 2010]. Candidate formations are bound by impermeable "caprock" layers that prevent the buoyant rise of the injected $\mathrm{CO}_{2}$ [Widjajakusuma et al., 1999; Kneafsey and Pruess, 2010; Wollenweber et al., 2010]. This type of physical trapping by an impermeable layer is only one of several major types of trapping that could lead to permanent storage of $\mathrm{CO}_{2}$ in the subsurface. A second mechanism by which $\mathrm{CO}_{2}$ can become permanently immobilized is the dissolution of $\mathrm{CO}_{2}$ into pore water. $\mathrm{CO}_{2}$ will dissolve into the pore water creating carbonic acid and carbonate species at a concentration that is a function of temperature, pressure and pore water composition. This $\mathrm{CO}_{2}$ will remain in the pore water, which usually has migration rates on the order of $\mathrm{cm}$ or $\mathrm{m}$ per year. A third trapping mechanism involves the precipitation or mineralization of dissolved carbonates. Dissolved carbonates are known to react with various divalent cations to form metal carbonates which would fall out of solution. Figure 1.1 presents a schematic of these trapping processes in the context of a $\mathrm{CO}_{2}$ plume developing in the subsurface. 


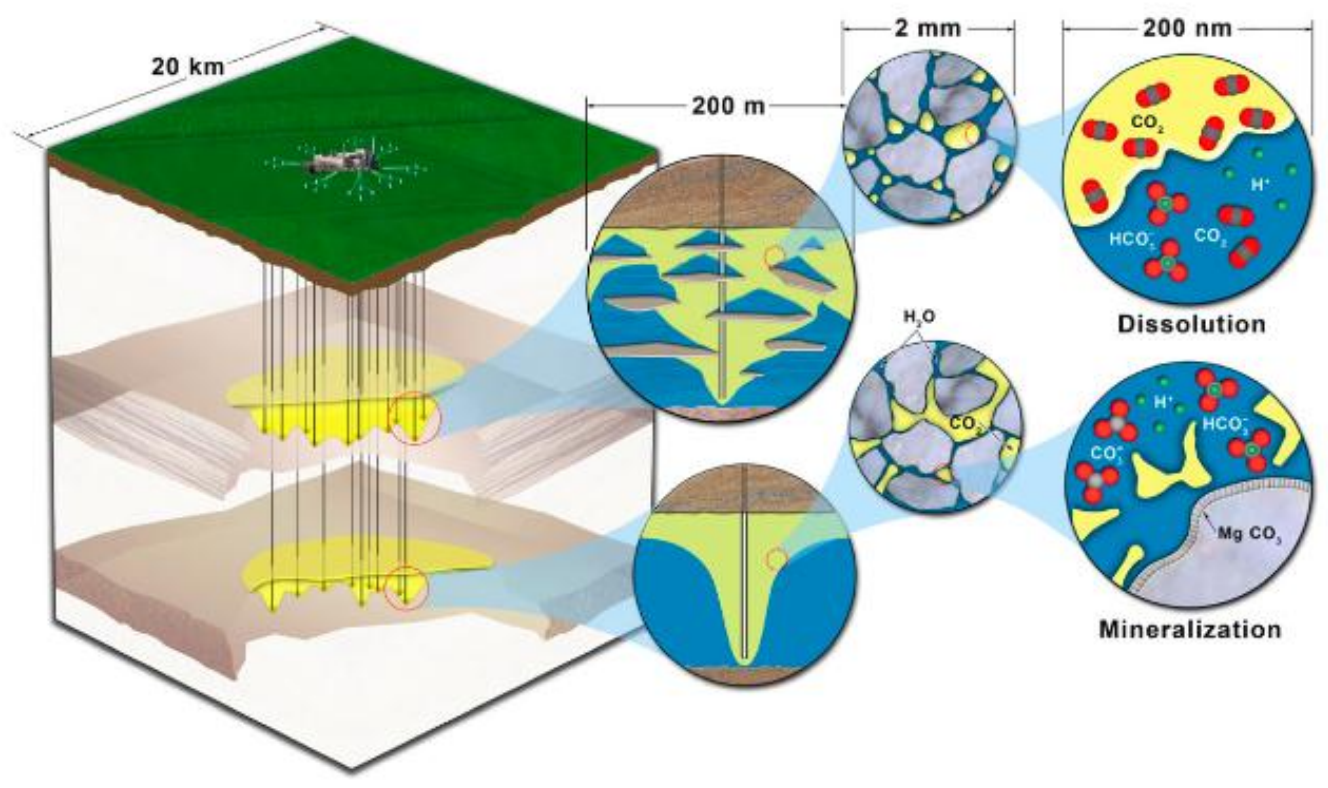

Figure 1.1. Schematic of $\mathrm{CO}_{2}$ sequestration showing $\mathrm{CO}_{2}$ plumes (yellow), inserts depict propose trapping processes $[M I T, 2007]$.

In the deep subsurface the phase behavior of $\mathrm{CO}_{2}$ will impact much of the relevant geochemistry and geophysics related to GCS processes. The trapping mechanisms described above, particularly dissolution and mineralization, are greatly affected by the solubility and chemical reactivity of dissolved $\mathrm{CO}_{2}$. Figure 1.2 shows the relationship between pressure, depth, temperature, and $\mathrm{CO}_{2}$ density and phase behavior. Notice that as $\mathrm{CO}_{2}$ rises through the subsurface, it will follow constant density gradients that in most cases (depending on the ground temperature and particular thermal conditions of the region) will pass through the critical point of $\mathrm{CO}_{2}$. The relationship between $\mathrm{CO}_{2}$ phase behavior, temperature, pressure, and the composition of the aquifer formation and brine are all crucial for understanding how likely $\mathrm{CO}_{2}$ will stay in the subsurface. 


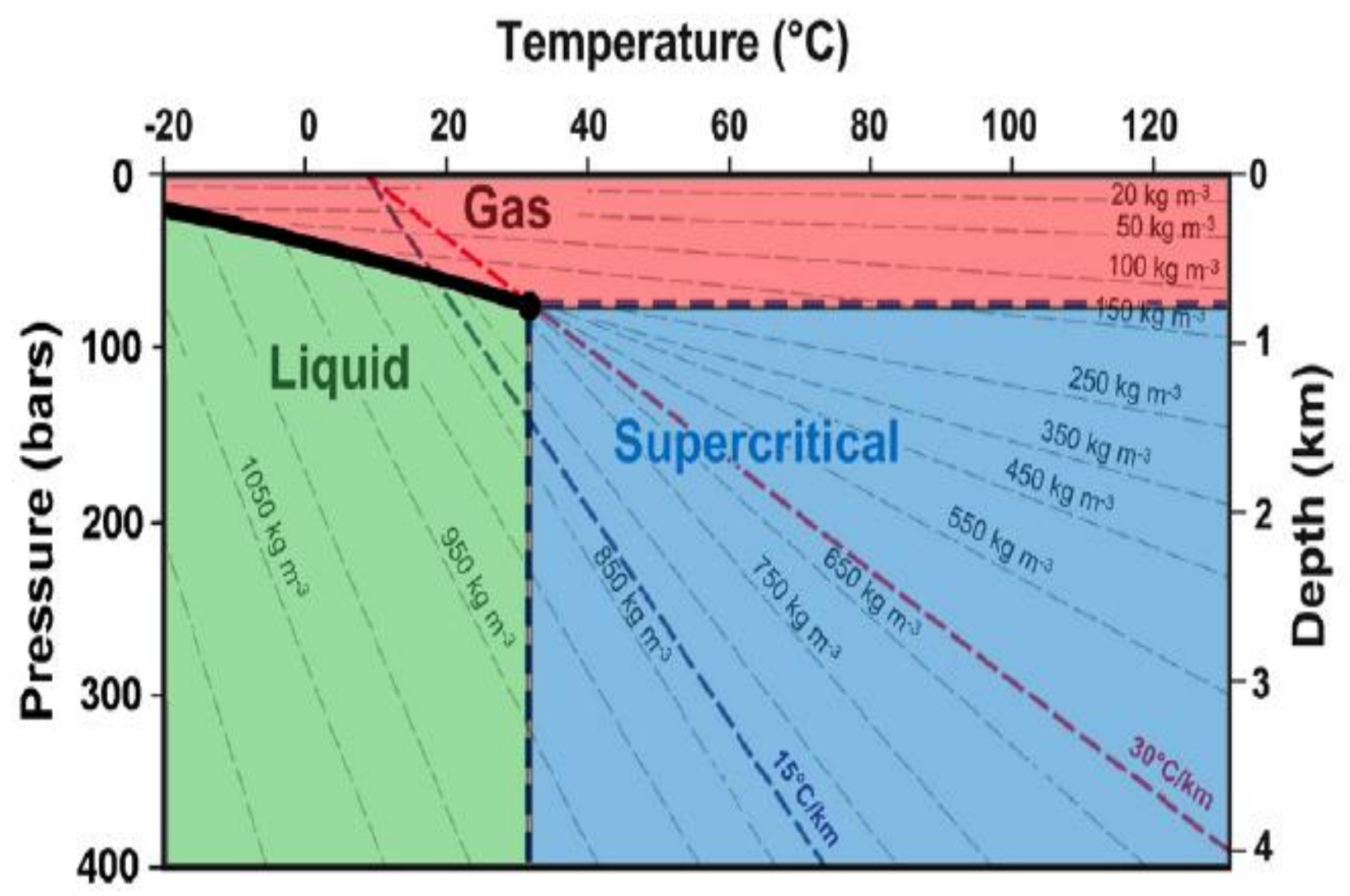

Figure 1.2. Phase diagram for $\mathrm{CO}_{2}$ showing pressure - temperature $-\mathrm{CO}_{2}$ density profiles that exist in the subsurface with two geothermal gradients highlighted [Oldenburg and Lewicki, 2005].

There are several key criticisms of GCS. Some argue that it does not represent a true solution to the carbon problem and that we need to instead wean ourselves from fossil fuels to focus instead on renewables and nuclear. These critiques ignore the fact that moving toward a carbon neutral energy infrastructure will take decades. Others are concerned about the induced seismicity impacts of injected high-pressure fluid into geologic formations. Even though more work is needed to understand this, preliminary work suggests that almost all the induced seismicity is very low energy and would not impact human infrastructure at the surface. The biggest practical obstacle to GCS is its high cost and this will not be overcome until there is a carbon market in place that values the sequestration potential that only GCS can offer. 


\subsection{Problem Statement}

For GCS to be successful, $\mathrm{CO}_{2}$ leakage rates must be minimized. The vertical migration of $\mathrm{CO}_{2}$ plumes from deep repositories in the subsurface is a poorly understood process. The few modeling studies published to date on this topic suggest that both self-enhancing flow and selflimiting flow are possible [Pruess, 2008a; Silin et al., 2009]. Eruptions from natural $\mathrm{CO}_{2}$ reservoirs and from man-made well bores, provide evidence for the possibility of high-energy discharges of $\mathrm{CO}_{2}$ from deep origins fueling concerns about the risk implications of GCS [Pruess, 2008b; Nordbotten et al., 2004]. At present, the uncertainty and risk associated with leakage processes is one of the most significant roadblocks to widespread implementation of the technology. Field-scale demonstrations generally do not provide an opportunity to study leakage because they are at small scales and the sites are too poorly characterized. Additionally, these projects are designed to be successful and not include leakage events, and leakage would be expected to occur over long time scales in heterogeneous formations where it is difficult to establish a relationship about cause and effect. In the lab, these processes are difficult to study because experiments of flow through porous media at high pressure do not easily scale down and the heterogeneities in the subsurface make it challenging to develop first-principle models of leakage under GCS conditions.

The densities of $\mathrm{CO}_{2}$ under all phase states are lower than that of the connate brines and so a parcel of $\mathrm{CO}_{2}$ will be subject to buoyant forces. If $\mathrm{CO}_{2}$ leaks out of its target formation toward the ground it could involve multiple pathways such as heterogeneities and fractures in the bounding formation, groundwater flow to shallower unconfined aquifers faults, improperly plugged abandoned wells, etc. as shown in Figure 1.3. Leakage is especially difficult to characterize because of the very large length scales involved and the numerous heterogeneities in geologic for- 
mations involved, the chemistry of the brines, and the wide range of shear, temperature and pressure conditions that result in complex solubility phase and interfacial behavior. The fundamental processes driving $\mathrm{CO}_{2}$ leakage from deep subsurface porous formations are still very poorly understood [Oldenburg and Lewicki, 2006]. In particular, a lack of experimental data on important parameters impedes efforts to develop first-principle models of leakage under GCS conditions.

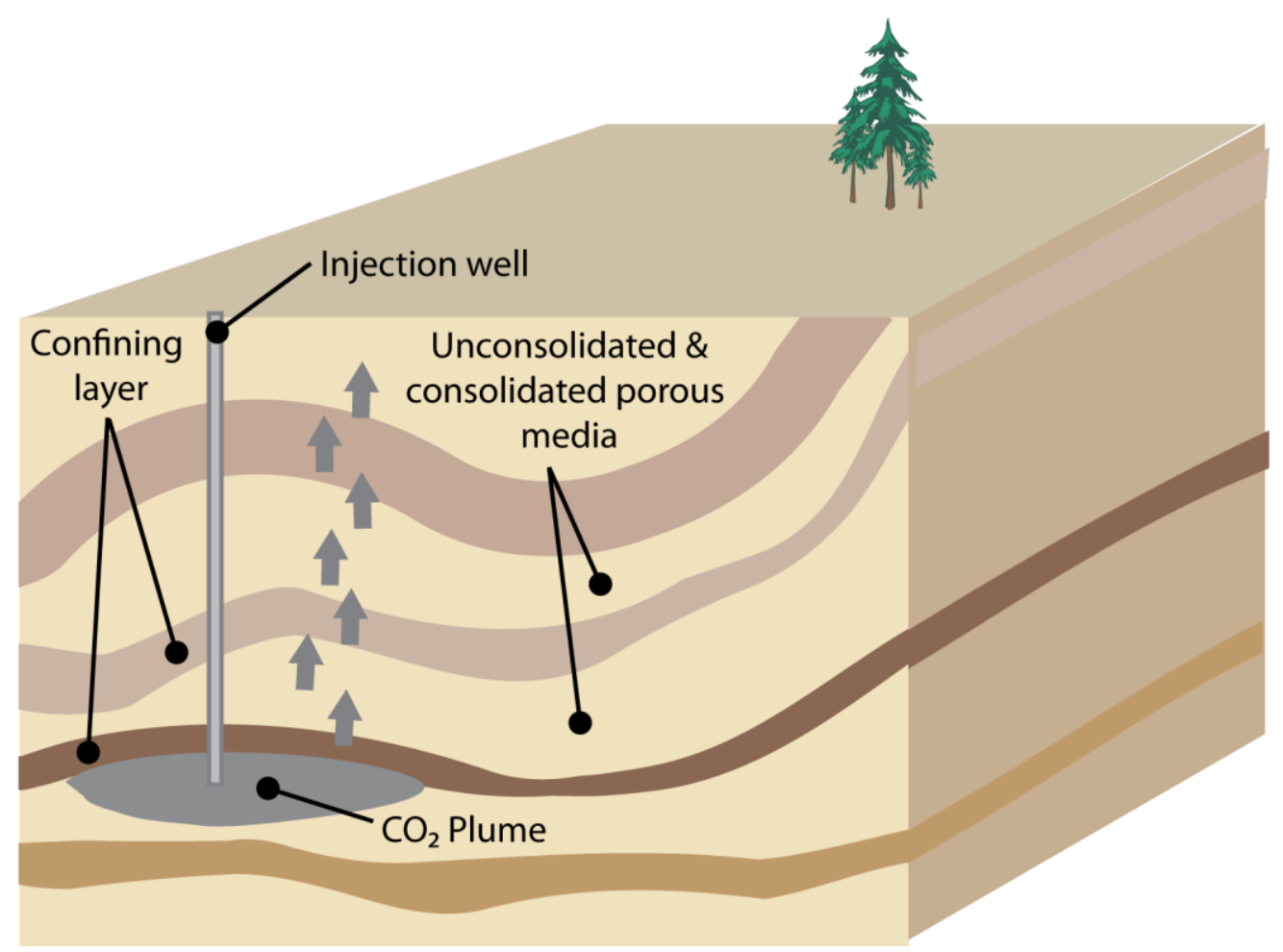

Figure 1.3. Leakage from geologic carbon sequestration sites will involve $\mathrm{CO}_{2}$ vertical transport through numerous subsurface formations 


\subsection{Research Objectives, Scope and Overview of Dissertation}

\subsubsection{Research Objectives and Scope}

The objective of this work is to develop an improved understanding of how $\mathrm{CO}_{2}$-brinemineral interfacial properties might impact buoyancy driven flow through porous media. This understanding is crucial for developing a clearer picture of how leakage might play a role in geologic carbon sequestration. Rheological properties of single and multiple phase $\mathrm{CO}_{2}$-brine mixtures and the wettability of representative rock and clay species in contact with $\mathrm{CO}_{2}$ and connate brine were characterized experimentally over a range of GCS-relevant temperature, pressure, $\mathrm{pH}$, ionic strength, and shear conditions. A number of interesting trends emerged. Adhesion on certain mineral surfaces was observed and this could have an important impact on trapping mechanisms. The underlying phenomena is most likely attributed to the van der Waals forces affected by the structure of water films that form at the interface in between the mineral and the $\mathrm{CO}_{2}$ and the surface smoothness that favors intermolecular adhesion.

The experimental results collected here were incorporated into an existing modeling framework of $\mathrm{CO}_{2}$ leakage. The model was expanded to include better estimates of the shear forces and interfacial tension forces. This modeling framework sets the stage for validation experiments in columns that are the follow-up work from this thesis. Collectively, this work will reduce the uncertainty associated with selection of injection sites and long-term stewardship of these areas. It will also contribute to the 2010 Presidential directive of overcoming the barriers to widespread and cost-effective deployment of GCS within ten years, which will help position the United States as a leader in the global clean energy race. 


\subsubsection{Overview of Dissertation}

This dissertation is divided into seven chapters. Chapter 2 summarizes the state of the art in GCS and the geochemistry and geophysics that underlie the technology. The risk of carbon leakage and associated environmental impacts are discussed first and that is followed by an overview of the trapping mechanisms, which could effectively mitigate leakage once it ensues. Forces applied on rising discrete $\mathrm{CO}_{2}$ bubbles/droplets and continuous $\mathrm{CO}_{2}$ channel flow through porous media in deep saline aquifers are described. The interfacial phenomena that could impact leakage including the rheological, wettability and adhesion behaviors involved in this $\mathrm{CO}_{2}$ and brine transport process are discussed. A brief introduction to relevant modeling efforts is provided.

Chapter 3 presents the results of the $\mathrm{CO}_{2}$-brine rheology study and its implications to GCS. The rheological characteristics of the $\mathrm{CO}_{2}$-brine mixtures under fluid-liquid equilibrium (FLE) and vapor-liquid equilibrium (VLE) conditions under the GCS schemes are described. The linear and nonlinear responses of $\mathrm{CO}_{2}$ bubbles/droplets to shear are characterized. The thixotropic and time dependent properties of VLE $\mathrm{CO}_{2}$-brine mixtures are also quantified. These Newtonian and non-Newtonian rheological data are fitted into theoretical models to predict rheological effects under broader GCS scenarios. The experimental data and constitutive relationships for leaked $\mathrm{CO}_{2}$ have important implications to the success of GCS and will lead to better capacity to prevent leakage.

In Chapter 4, wettability phenomena at the $\mathrm{CO}_{2}$-brine-mineral interface are studied on a variety of commonly encountered reservoir minerals. The effects of $\mathrm{pH}$, ionic strength, level of carbonation, mass transfer, instantaneous phase change inside $\mathrm{CO}_{2}$ droplets, surface roughness and similarities in crystal structure and molecular formula on equilibrium contact angle and its 
hysteresis are also characterized. All minerals were found to be highly $\mathrm{CO}_{2}$-nonwetting. $\mathrm{pH}$ and ionic strength exhibit noticeable influence on wettability. Advancing and receding contact angle are measured to characterize the hysteretic effects associated with wettability. Although the alteration of wettability lies in a narrow range, the scaling up impacts on trapping capacity are discernible and have very important implications to GCS.

Chapter 5 is focused on the interfacial adhesion between $\mathrm{CO}_{2}$ and certain minerals such as trioctahedral phlogopite mica, amorphous silica and calcite. When adhesion occurs, $\mathrm{CO}_{2}$ droplets/bubbles become strongly bonded to the solid surfaces and are not easily removed under turbulence. Adhesion results in a significant alteration of wettability spanning non-wetting to intermediate wetting to wetting. It also causes significant increase in ternary phase lines and obvious distortion of bubble geometry. The strength of adhesion is evaluated using the advancing and receding cycles on captive $\mathrm{CO}_{2}$ droplets. Extensive adhesive energy is observed when $\mathrm{CO}_{2}$ droplets are under tensile stress and get highly deformed. The interfacial adhesion phenomenon is found to be caused by nanometer scale short-range van der Waals forces and is strongly influenced with $\mathrm{pH}$ and ionic strength.

Two modeling frameworks incorporating the experimental results of the rheology, wettability and adhesion studies have been employed to improve the accuracy of $\mathrm{CO}_{2}$-brine multiphase flow through porous media simulation in Chapter 6. One is based on the well-known Ergun equation for the shear drag force and the other one is derived from first principles on transport of $\mathrm{CO}_{2}$ ganglions through porous media based on Darcy's law. Sensitivity analysis is conducted to evaluate the relative significance of important factors including contact angle, pore size, bubble size, grain size, porosity and permeability. Monte-Carlo analysis is conducted to 
calculate the overall traveling velocity of $\mathrm{CO}_{2}$ based on packages of field data from several $\mathrm{CO}_{2}$ storage sites.

Chapter 7 reiterates on the important findings of this research work. It also provides guidelines for on-going and future work including identifying more $\mathrm{CO}_{2}$-adhesive minerals, conducting molecular dynamics simulation to better understand adhesion mechanisms, improving the current modeling work and validating the models with short column experiments.

A flow chart of this dissertation is shown in Figure 1.4.

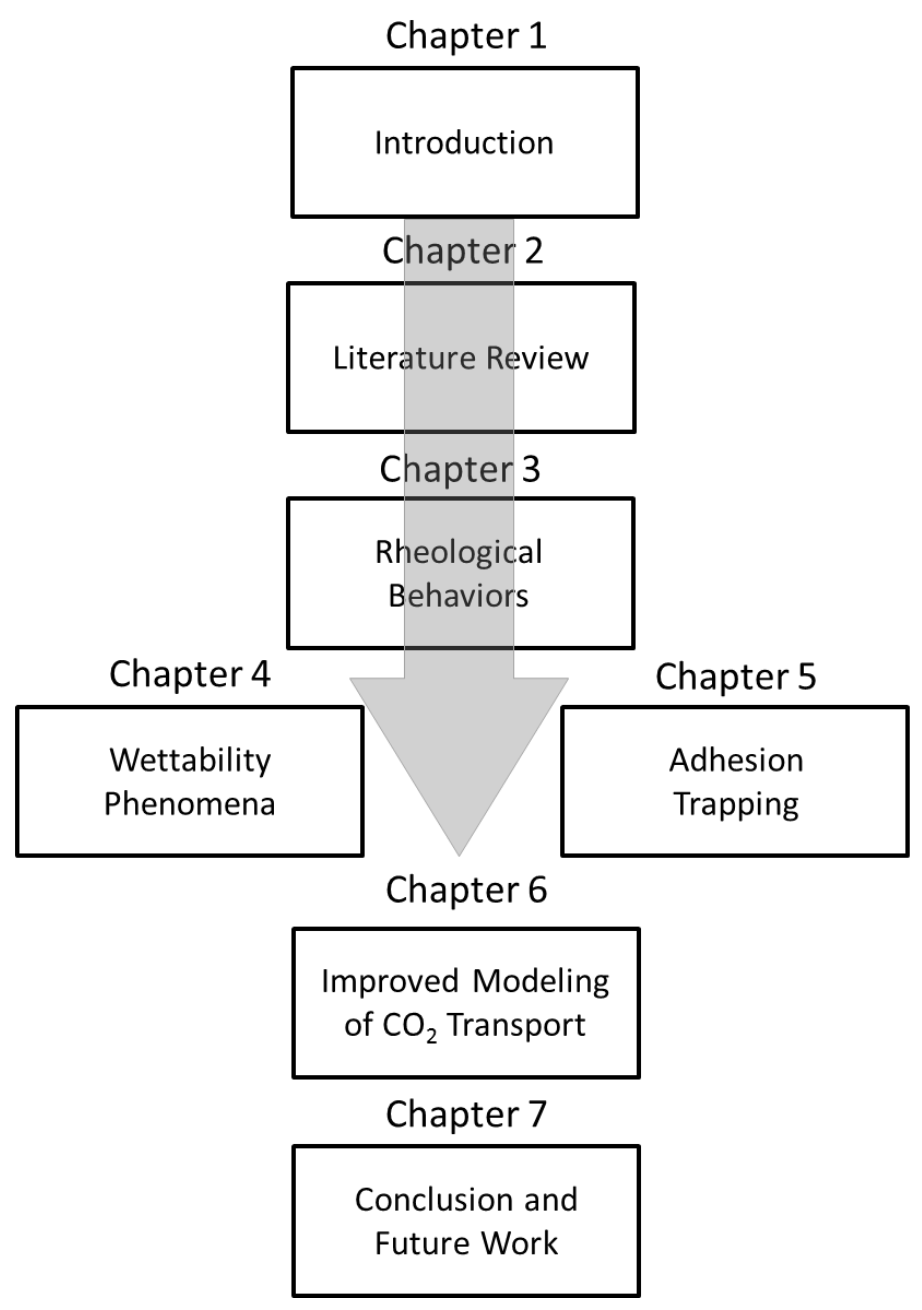

Figure 1.4. Overview of Dissertation 


\section{CHAPTER 2}

\section{Literature Review}

This chapter provides a brief summary of the state of the science with regard to the geophysics and geochemistry of $\mathrm{CO}_{2}$ rise in porous media. The role of $\mathrm{CO}_{2}$-brine mixture rheology, the wettability of rock and clay mineral surfaces and the interfacial adhesion phenomena on bubble transport behaviors is all discussed within this broader context of understanding buoyancy driven transport of $\mathrm{CO}_{2}$. Gaps in the existing knowledge are also presented.

Leakage from GCS sites remains poorly understood because it is so complex and because there is a disconnection between lab-scale experiments and pilot scale demonstration. Field projects aimed at understanding the fate of $\mathrm{CO}_{2}$ and developing monitoring technologies are under way today [Bhattacharya, 2011]. These projects are only being carried out at the demonstration scale in well characterized formations. Few have attempted to use what is known about the properties of these formations to establish cause-and-effect relationships [Silin et al., 2009]. Third, there is a lack of laboratory-scale experimental data of the key parameters including viscosity, contact angle and interfacial tension to be able to understand the limited field data that is available. This section provides a brief overview of some of the gaps in understanding associated with leakage from GCS sites. 


\subsection{Geologic Carbon Sequestration (GCS)}

GCS has been under development for over two decades [Chen and Zhang, 2010; Yang and $G u, 2006]$. The unit operations needed for transporting and pumping $\mathrm{CO}_{2}$ into aquifers in geologic formations have been developed by the petroleum industry for Enhanced Oil Recovery (EOR) applications [Lake, 1989]. Large-scale naturally-occurring deposits of $\mathrm{CO}_{2}$ have been discovered around the world suggesting that long-term storage of $\mathrm{CO}_{2}$ in the subsurface is technically feasible. Calculations of storage capacity suggest that industrialized nations have the capacity to store waste $\mathrm{CO}_{2}$ in confined sedimentary basins for at least several decades and up to several centuries [Bruant et al. 2002; MIT, 2007; Benson and Cole, 2008]. Although GCS is the best candidate for carbon mitigation, there is limited experience integrating individual components or implementing on large scale.

At the depths considered for $\mathrm{CO}_{2}$ injection and storage (below $\sim 1 \mathrm{~km}$ ), hydrostatic forces produce pressures greater than 100 bars, and thermal gradients result in temperatures greater than $35^{\circ} \mathrm{C}$ [Oldenburg and Lewicki, 2006; Lackner, 2003]. In GCS, $\mathrm{CO}_{2}$ would exist mostly in the supercritical state, having a density close to that of liquid $\mathrm{CO}_{2}$ and compressibility close to that of gaseous $\mathrm{CO}_{2}$. The density of $\mathrm{CO}_{2}$ under these conditions is lower than the density of the native brines and so the parcel of $\mathrm{CO}_{2}$ will be subject to upward buoyant forces. These conditions are not far from the critical point of $\mathrm{CO}_{2}$ (at 74 bar and $31^{\circ} \mathrm{C}$ ), so there is significant variation in $\mathrm{CO}_{2}$ density over relatively short length scales. 


\subsection{Trapping Processes}

In a broad sense, leakage processes of interest for GCS applications involve all those in which $\mathrm{CO}_{2}$ escapes from the reservoirs into which it is originally injected. To understand these pathways, it is first useful to consider a variety of trapping processes/mechanisms wherein $\mathrm{CO}_{2}$ becomes trapped in the deep-subsurface saline aquifers [Bachu, 2008]. After injection, supercritical/liquid $\mathrm{CO}_{2}$ will stay on top of connate brine as a discrete phase due to buoyancy and it will be trapped underneath low-permeability caprocks. This is known as structural and hydrodynamic trapping and will prevent buoyant rise of the $\mathrm{CO}_{2}$ over short time scales. Similarly, when the $\mathrm{CO}_{2}$ reaches dead-end pore spaces in the porous media, capillary trapping will occur [Bachu, 2008; Mansoori et al., 2009]. Over longer times scales, $\mathrm{CO}_{2}$ will dissolve into formation brines forming carbonic acid and water-borne carbonates and reach equilibrium that is a function of pressure and temperature, defined as solubility trapping. Precipitation of the dissolved silicate and carbonate materials rich in $\mathrm{Ca}, \mathrm{Mg}$ and $\mathrm{Fe}$, etc. with cationic species in the formation brines will constitute permanent mineral trapping. Sandstone aquifers rich in glauconite, illite, anorthite, chlorite and smectite minerals and low in carbonates favor this process [Bachu, 2008]. Finally, $\mathrm{CO}_{2}$ will be retained by adsorption trapping in which $\mathrm{CO}_{2}$ adsorbs to mineral surfaces of the host formation.

For reservoirs with a shaly/sandstone caprock, the capillary-sealing potential of that formation is a critical factor for trapping. This seal potential can be quantified by the capillary pressure $P_{C}$ which is an indicator of the threshold reservoir over pressure that the brine-saturated caprock can provide. This potential can be also be expressed in terms of a maximum storage

pressure of $\mathrm{CO}_{2}$, equal to the hydrostatic pressure in the caprock plus the $\mathrm{CO}_{2}$ capillary entry pressure in the brine-saturated caprock/porous media, as shown in Eq.2.1. 


$$
P_{\mathrm{CO}_{2}}=P_{C}+P_{\text {brine }} \quad \text { Eq. } 2.1
$$

Where $P_{\mathrm{CO}_{2}}$ and $P_{\text {brine }}$ are the pressure of $\mathrm{CO}_{2}$ an brine in pores, respectively; $\boldsymbol{P}_{C}$ is the capillary entry pressure, once $P_{C}$ is exceeded, buoyancy driven flow of $\mathrm{CO}_{2}$ could initiate.

\subsection{Buoyancy-Driven $\mathrm{CO}_{2}$ Transport through Porous Media}

Multiphase flow in porous media is a very well-studied process in different applications including oil and gas extraction and environmental remediation of aquifers. Efforts to build on this knowledge to understand buoyancy-driven rise of $\mathrm{CO}_{2}$ through porous media are, to date, derived from theoretical simulations that predict outcomes ranging from self-enhancing flow to self-limiting flow [Pruess, 2008a; Silin et al., 2009]. The viscosity contrast between a rising parcel of $\mathrm{CO}_{2}$ and the brine it is displacing increases making it more likely to develop instabilities and fingering [Riaz and Tchelepi, 2008; Patzek et al., 2003]. This process is further complicated by permeability and capillarity associated with the porous media and the sensitivity of the fluid wettability to variations in mineral surfaces (e.g. rock vs. clay minerals) and the $\mathrm{pH}$-dependence of this wettability. Moreover, it is also very challenging to study reactions and multiphase flow in an integrated way, under high-pressure conditions, over realistic length scales. An accurate model must connect molecular-scale computer simulation, pore-scale, bench-scale and intermediate-scale experiments and field-scale injection demonstrations.

Buoyancy-driven transport of $\mathrm{CO}_{2}$ is initiated by density as well as a number of other factors including formation permeability and chemistry. To understand this complex behavior, it is useful to consider the case of discrete $\mathrm{CO}_{2}$ bubbles, where rise can be understood in terms of the 
force balance that impacts capillary trapping and dissolution/precipitation reactions [ $L i$ et al., 2006]. The rise of $\mathrm{CO}_{2}$ parcel in porous media is expected to occur in dynamic response to three main acting forces: namely, buoyant force $\mathrm{F}_{\mathrm{b}}$, interfacial tension force $\mathrm{F}_{\mathrm{st}}$ and shear drag force $\mathrm{F}_{\mathrm{d}}$ [Corapcioglu et al., 2004; Oldenburg and Lewicki, 2006]. Buoyancy force provides the fundamental driver for $\mathrm{CO}_{2}$ to rise, and interfacial tension force and drag force resist the motion (Figure 2.1). Once polydisperse bubbly flow $\left(R_{\mathrm{CO}_{2}}<R_{P}\right)$ or channel flow $\left(R_{\mathrm{CO}_{2}} \geq R_{P}\right.$, where $R_{\mathrm{CO}_{2}}$ is the radius of $\mathrm{CO}_{2}$ bubble and $R_{P}$ is the equivalent radius of a pore throat in the porous media) conditions ensue, the smaller bubbles may still impact the flow, but the interfacial tension forces and the filtration of bubbles associated with the contact with the geologic formation will come into play. The interfacial tension between $\mathrm{CO}_{2}$ and brine or water under high-pressure conditions is too low to support the propagation of small bubbles [Hebach et al., 2002]. The pressure inside the $\mathrm{CO}_{2}$ bubble, described by Henry's Law must still exceed the sum of the hydrostatic pressure and the interfacial tension between $\mathrm{CO}_{2}$ and brine [Crandell et al., 2010]. 

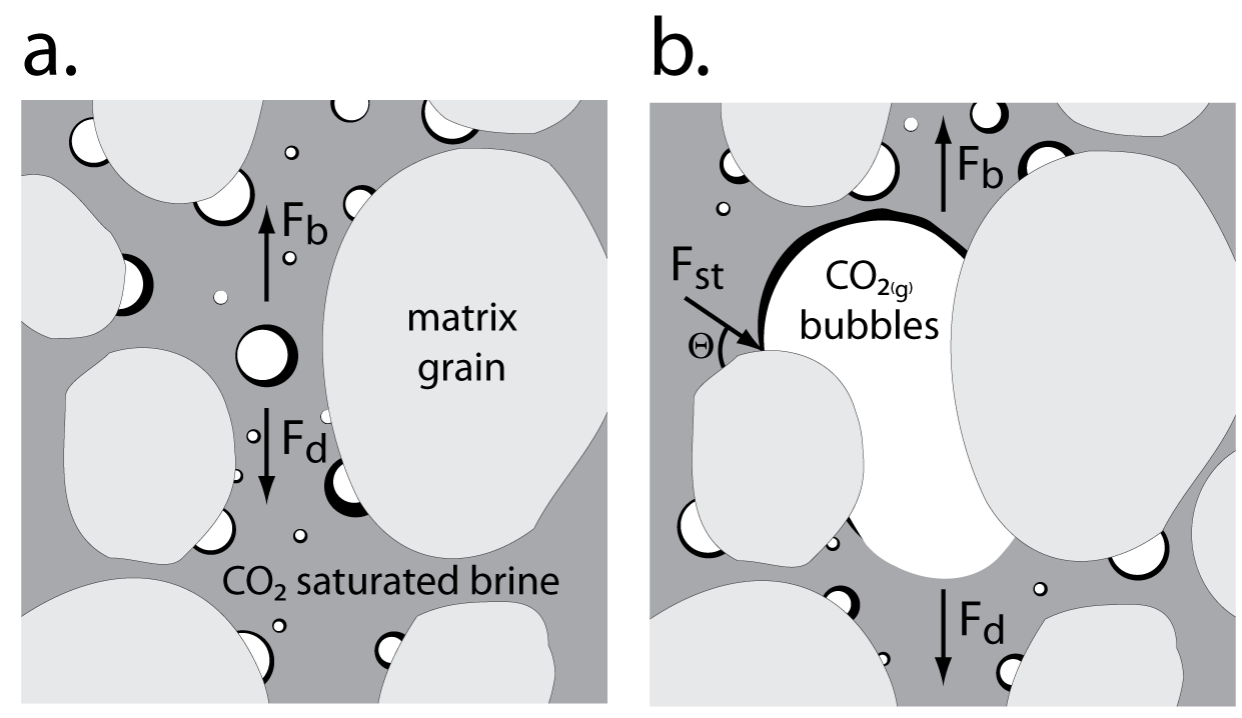

Figure 2.1. Force balance on bubbles of $\mathrm{CO}_{2}$ rising through porous media when a. $R_{\mathrm{CO}_{2}}<R_{P}$ and b. $R_{\mathrm{CO}_{2}} \geq R_{P}$. Buoyant forces $F_{b}$ and drag forces $F_{d}$ act under both scenarios whereas interfacial tension forces $F_{\text {st }}$ act only on those bubbles that contact the pore walls.

Current GCS models of $\mathrm{CO}_{2}$ transport through porous sediments do not account for geochemical interactions in caprocks or the effect of compressibility of $\mathrm{CO}_{2}$ bubbles. This is because: (1) sometimes it is reasonable to ignore acid-driven reactions, such as in siliciclastic formations with shaly caprocks, (2) there is a desire to keep models simple, which is computationally hard to do when water-rock interactions must be described, and (3) it is difficult to do so given uncertainties about reaction kinetics and the effects on porosity and permeability. Besides, a rising bubble of $\mathrm{CO}_{2}$ is governed by a different set of physics from a rising parcel of $\mathrm{CO}_{2}$ large enough to rise as a front that can "finger" if the viscosity contrast is large enough. This behavior may be advantageous in the context of leaked $\mathrm{CO}_{2}$ because it increases the likelihood of residual saturation trapping and dissolution in brine. 
Published efforts to develop these models have made simplifying assumptions about the properties of the liquid or the gas phases that can, in some instances degrade the quality and accuracy of these models [Oldenburg, 2007b]. Limited experimental data exists on the contact angle between $\mathrm{CO}_{2}$ and most of the solid minerals that a leak is likely to encounter. To better understand how quickly and where a parcel of $\mathrm{CO}_{2}$ is likely to escape, better modeling tools are needed. These tools must be based on experimental results collected under GCS-relevant conditions.

\subsection{Leakage from GCS Sites}

The benefits of GCS would be diminished if $\mathrm{CO}_{2}$ leaked to the atmosphere in appreciable amounts. Leakage would not only translate into a loss of carbon mitigation credits (or the need to pay carbon emission fees and taxes), it would also contribute to greenhouse gas (GHG) emissions because of the energy consumed and the net carbon footprint in the failed $\mathrm{CO}_{2}$ capture and storage operation [Pollak and Wilson, 2009; Dooley et al., 2006]. Leakage may also have environmental and security consequences, such as well and pipeline blowouts, alternation of water and soil $\mathrm{pH}$ and contamination of non-climate-related water resources and associated damages to ecosystems, or worse, mobilization of toxic substances and dangerous displacement of oxygen at the land surface [Birkholzer and Zhou, 2009; Gasda, 2011]. Eruptions from naturally occurring $\mathrm{CO}_{2}$ reservoirs and from man-made well bores provide evidence for the possibility of highenergy discharges of $\mathrm{CO}_{2}$ from deep origins, though no eruptions of this type have been observed in a GCS context [Pruess, 2008b, Oldenburg et al., 2010]. A Department of Energy requirement of no greater than $0.01 \%$ rate of leakage every year must be fulfilled [IPCC, 2005] to ensure effective implementation of GCS projects. 
As $\mathrm{CO}_{2}$ plumes rise in response to the geothermal and hydrostatic gradients that exist in the subsurface, phase separation in supersaturated brines may occur as the pressure or temperature drops. The phase behavior of $\mathrm{CO}_{2}$-brine mixtures is of primary importance in predicting conditions under which bubbles form, as well as the driving force (density difference) for their upwards motion. Figure 2.2 illustrates how the phase behavior of $\mathrm{CO}_{2}$ interacts with depth and the natural geothermal gradients that exist in the subsurface. For $\mathrm{CO}_{2}$ injected at similar depths at different sites, indicated with dashed lines a and b in Figure 2.2 (the dark black circles represent the $\mathrm{CO}_{2}$ reservoirs) and the detailed thermophysical properties listed in Table 2.1, the buoyant forces on a rising parcel of $\mathrm{CO}_{2}$ may grow due to the increased buoyancy that comes from expansion of $\mathrm{CO}_{2}$ from supercritical (i.e., $\mathrm{CO}_{2(\mathrm{sc})}$ ) to subcritical conditions (i.e., either $\mathrm{CO}_{2(1)}$ or $\mathrm{CO}_{2(\mathrm{~g})}$ ) [Oldenburg, 2007a; Pruess, 2008b]. The horizontal lines indicate the relationship between density and phase behavior at the five depths. As the $\mathrm{CO}_{2}$ moves toward the surface, the density decreases appreciably and the buoyant driving force on $\mathrm{CO}_{2}$ keeps growing during the entire process. The density data presented here shows how the phase characteristics of $\mathrm{CO}_{2}$ could result in nonlinear differences between the density of the brine and the $\mathrm{CO}_{2}$. In some cases, the $\mathrm{CO}_{2}$ will transition directly from supercritical to gas phase resulting in a drop in density and an increase in the difference between the density of the brine and the $\mathrm{CO}_{2}$ (path a). In other cases, the density of the fluid will increase as it transitions from supercritical to liquid form followed by a sharp decrease when transitioning to the gas phase (path b). This oscillation in density will result in a dip in the buoyant driving force on $\mathrm{CO}_{2}$.

Density is not the only characteristic that matters in understanding leakage rates; permeability and capillarity associated with the porous media, which are site specific, will play a major role. In addition, if the parcel rises quickly, adiabatic expansion and associated Joule-Thomson 
cooling would tend to mitigate the density difference [Pruess, 2008a]. $\mathrm{CO}_{2}$ may lose its mobility if it gets "left behind" as residual saturation (i.e., capillary trapping) [Kumar et al., 2005; Juanes et al., 2006; Taku Ide et al., 2007].

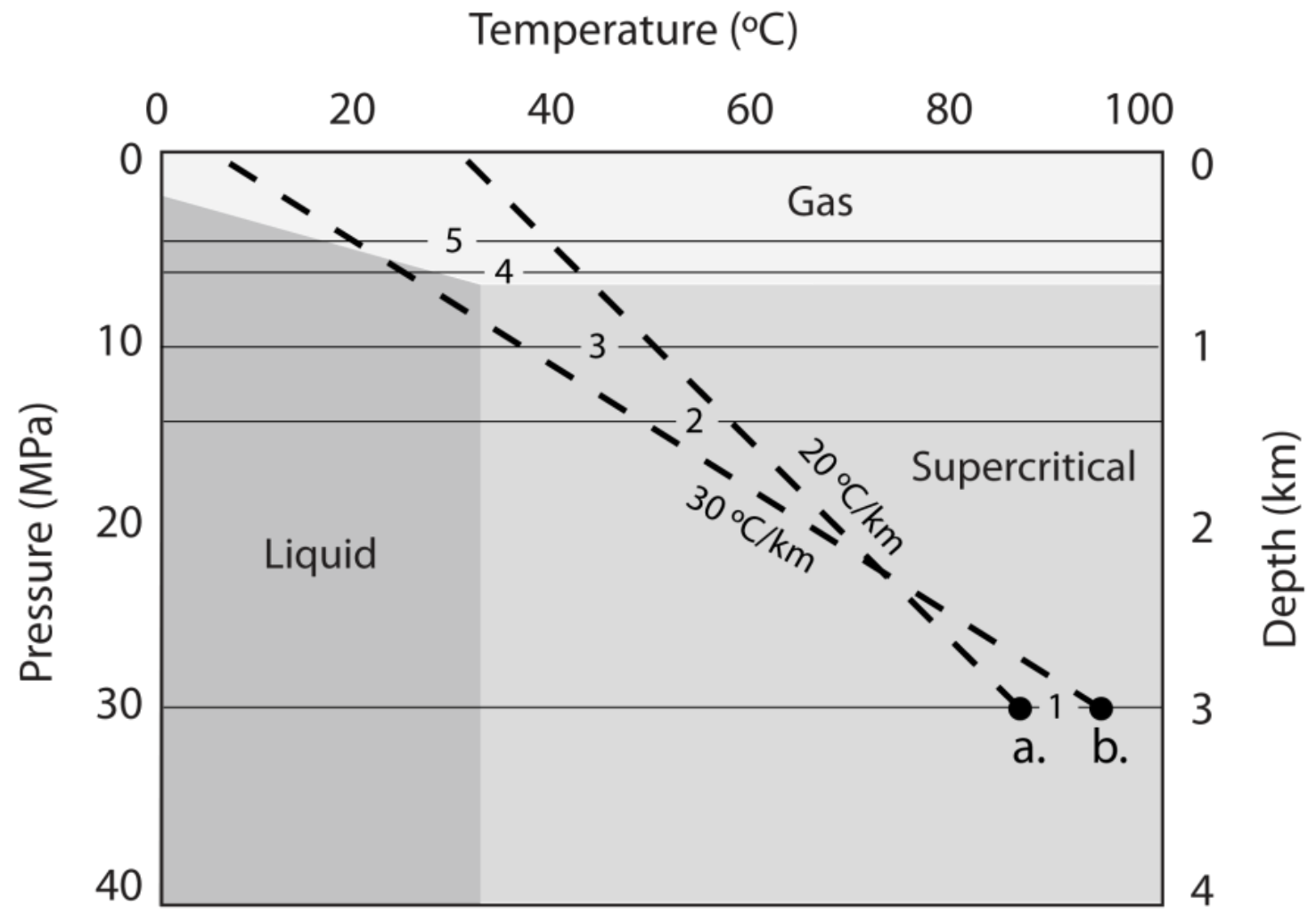

Figure 2.2. Phase behavior of $\mathrm{CO}_{2}$ in the subsurface is a function of the depth and the thermal gradient in the subsurface. a. and b. correspond to two possible leakage pathways for a parcel of $\mathrm{CO}_{2}$ that would have implications for the phase behavior and density conditions. The horizontal numbered lines illustrate how $\mathrm{CO}_{2}$ density/phase behavior will impact leakage. 
Table 2.1. Representative pressure conditions for leaking $\mathrm{CO}_{2}$ under two geothermal gradients. Nonlinear differences in density driven by the $\mathrm{CO}_{2}$ phase characteristics in pathways a and $\mathrm{b}$ could result in complex flow behavior (as illustrated by the $\Delta$ Specific Weight term). The \# in the first column corresponds to the depths presented in Figure 2.1. Phase definitions are SC = supercritical, $\mathrm{L}=$ liquid, and $\mathrm{V}=$ vapor. $\mathrm{T}=$ temperature $\left({ }^{\circ} \mathrm{C}\right)$ and $\mathrm{D}=$ depth $(\mathrm{km})$.

\begin{tabular}{|c|c|c|c|c|c|c|c|c|c|c|}
\hline & \multicolumn{6}{|c|}{ Leakage pathway a $(T=30+20 D)$} & \multicolumn{4}{|c|}{ Leakage pathway $\mathrm{b}(T=5+30 D)$} \\
\hline \# & $\begin{array}{l}\text { Pres. } \\
\text { (MPa) }\end{array}$ & $\begin{array}{l}\text { Depth } \\
(\mathrm{km})\end{array}$ & $\begin{array}{l}\text { Temp } \\
\left({ }^{\circ} \mathrm{C}\right)\end{array}$ & $\begin{array}{l}\text { Density } \\
\left(\mathrm{kg} / \mathrm{m}^{3}\right)\end{array}$ & Phase & $\begin{array}{c}\Delta \text { Specific } \\
\text { Weight } \\
\left(\mathrm{N} / \mathrm{m}^{3}\right)\end{array}$ & $\begin{array}{l}\text { Temp } \\
\left({ }^{\circ} \mathrm{C}\right)\end{array}$ & $\begin{array}{l}\text { Density } \\
\left(\mathrm{kg} / \mathrm{m}^{3}\right)\end{array}$ & Phase & $\begin{array}{c}\Delta \text { Specific } \\
\text { Weight } \\
\left(\mathrm{N} / \mathrm{m}^{3}\right)\end{array}$ \\
\hline 1 & 30 & 3 & 90 & 703.27 & $\mathrm{SC}$ & $\begin{array}{l}973.0 \\
\end{array}$ & 95 & 682.39 & SC & -170.3 \\
\hline 2 & 15 & 1.5 & 60 & 604.09 & $\mathrm{SC}$ & 2155.8 & 50 & 699.75 & SC & -128.1 \\
\hline 3 & 10 & 1 & 50 & 384.33 & $\mathrm{SC}$ & 1947.4 & 35 & 712.81 & SC & -116.6 \\
\hline 4 & 7 & 0.7 & 44 & 185.81 & $\mathrm{~V}$ & 713.7 & 26 & 724.7 & $\mathrm{~L}$ & 5729.5 \\
\hline 5 & 5 & 0.5 & 40 & 113.05 & $\mathrm{~V}$ & & 20 & 140.65 & V & \\
\hline
\end{tabular}

\section{5 $\mathrm{CO}_{2}$ Solubility in Brines}

The solubility of $\mathrm{CO}_{2}$ in brines [Bachu and Adams, 2003; Portier and Rochelle, 2005; Duan et al., 2006] is a significant determinant of life span of a rising parcel of $\mathrm{CO}_{2}$. Limited experimental data are available for $\mathrm{H}_{2} \mathrm{O}-\mathrm{NaCl}-\mathrm{CO}_{2}$ mixtures at conditions relevant for GCS [Rumpf and Maurer, 1993]. Modeling of such systems using conventional equations of state is possible if sufficient experimental data are available to determine the interaction parameters present in the equations of state. However, especially in the critical region, equations of state can be inaccurate [DePaolo and Orr, 2008]. The presence of high concentrations of mixed electrolytes poses additional challenges to the development of accurate equations of state valid over broad ranges of 
temperature and pressure. In particular, thermodynamic correlations fitted to experimental data at low concentrations often fail to describe the behavior at high salt loadings, especially at elevated temperatures and pressures [ Ji et al., 2007]. Finally, the transport properties of the mixtures of interest cannot be predicted from equations of state.

Published work has demonstrated that the phase behavior of $\mathrm{CO}_{2}-\mathrm{H}_{2} \mathrm{O}-\mathrm{NaCl}$ mixtures can be modeled accurately using Monte Carlo simulations [Vorholz et al., 2004]. Additionally, since $\mathrm{CO}_{2}$ mole fraction is controlled by the partial pressure of $\mathrm{CO}_{2}$ in equilibrium with the aqueous phase, the exact composition of the $\mathrm{CO}_{2}$-brine solutions was calculated using the semi-empirical method described by Bando et al. [2003]. These curves were used to identify both the composition of the mixture and the pressure at which phase separation would occur. All of the rheological tests in this study were conducted for pressures at or below $10 \mathrm{MPa}$, since this is the range most relevant for leakage processes and the least characterized in the existing literature. All of the wettability, adhesion and modeling work in this research accounted pressures in both low and high ends to cover cases in a broad range corresponding to the leakage and sequestration schemes. All experimental methods were also benchmarked using deionized (DI) water to ensure that the methods produced the expected behavior and to produce a comparison with the $\mathrm{CO}_{2}$ brine systems.

As leaked parcel of $\mathrm{CO}_{2}$ moves past confining layers and closer to the surface, the solubility of $\mathrm{CO}_{2}$ will drop resulting in the ebullition of small $\mathrm{CO}_{2}$ bubbles. The creation of multiplephase $\mathrm{CO}_{2}$ conditions occurs, in part, from the effects of Joule-Thomson cooling. In numerical studies performed by Pruess [2005], these effects are initiated by the decompression and cooling of rising supercritical $\mathrm{CO}_{2}$. This cooling leads to conditions approaching the $\mathrm{CO}_{2}$ saturation line, which causes the liquid to boil [Pruess, 2005]. This process is then followed by rapid expansion 
of gaseous $\mathrm{CO}_{2}$ as it rises and is subject to lower pressures. As $\mathrm{CO}_{2}$ in the aqueous phase is always present, this gives rise to the formation of a three-phase zone [Pruess, 2005]. These three phases interfere with one another through relative permeability effects. This strongly reduces fluid mobility locally as density increases to counter buoyancy forces and creates conditions where temperatures can recover from heat conduction from wall rocks, thus causing more liquid $\mathrm{CO}_{2}$ to boil [Pruess, 2005]. Thermodynamically, this will revert the system back to a two-phase state, increasing mobility until the system cycles back into a three-phase system, creating a semiperiodic cycle of self-enhancing and self-limiting vertical flow [Pruess, 2005]. These newly formed bubbles will migrate upwards and expand to compensate for the buoyancy change along the leakage path.

\subsection{Rheology of $\mathrm{CO}_{2}$-Brine Mixtures}

Viscosity of the interstitial bulk mixture of saline water in contact with $\mathrm{CO}_{2}$ is a key property that determines the shear drag force. Pruess [2006] notes that self-enhancement in vertical $\mathrm{CO}_{2}$ transport is possible after discharge due to the low viscosity and low density of $\mathrm{CO}_{2}$ when compared to the native brine. The lower viscosity could allow for a much larger volumetric flow rate for a given pressure gradient [Pruess, 2008a]. However, as effective mobility is a function of relative permeability and viscosity, $\mathrm{CO}_{2}$ effective mobility could actually decrease as well when considering conditions of multiple-phase $\mathrm{CO}_{2}$ flows even when a lower-viscosity fluid is introduced [Pruess, 2008a]. The only two existing publications to date on experimental characterization of the rheology of homogeneous $\mathrm{CO}_{2}$-brine mixtures have been carried out for high pressure $(\geq 10 \mathrm{MPa})$ and low shear conditions $\left(<10 \mathrm{~s}^{-1}\right)$ but can only study Newtonian behavior without investigating multiphase conditions under which $\mathrm{CO}_{2}$ bubbles exist and bubble deformation im- 
pacts flow through small pores by relying on falling capillary tube or solid particle viscometers where shear cannot be independently controlled [Bando et al., 2004; Kumagai and Yokoyama, $1998]$.

Many leakage processes will be expected to occur in shallower aquifers with hydrostatic pressure below $10 \mathrm{MPa}$ and the shear experienced by rising bubbles of $\mathrm{CO}_{2}$ in the subsurface spans a broad range on the order of 20 to $4000 \mathrm{~s}^{-1}$ using representative pore sizes and flow velocities (Table 2.2) [Juanes et al., 2006]. Under these conditions, complex rheology could ensue that would impact $\mathrm{CO}_{2}$ vertical transport. Geologists studying the flow of magma in the subsurface have recognized that the presence of multiple phases can result in complex non-Newtonian flow behavior [Llewellin et al., 2002b; Manga and Loewenberg, 2001]. The non-linear rheological behavior of $\mathrm{CO}_{2}$-brine mixtures has been reported in terms of interfacial behavior but not explored in detail for the bulk of the $\mathrm{CO}_{2}$-brine mixtures [Kloek et al., 2001; Tewes and Boury, 2005].

Table 2.2. The shear experienced by rising bubbles of $\mathrm{CO}_{2}$ in the subsurface is strongly influenced by the bubble velocity and geometry of the pores through which it is flowing.

\begin{tabular}{|c|c|c|}
\hline \multirow{2}{*}{$\underbrace{}_{\text {Diameter }(\mathrm{m}) \times 10^{-5}} \quad$ Velocity $(\mathrm{m} / \mathrm{s})$} & 0.001 & 0.02 \\
\hline & \multicolumn{2}{|c|}{ Shear Rate $\left(\mathrm{s}^{-1}\right)$} \\
\hline 2 & 200 & 4000 \\
\hline 4 & 50 & 1000 \\
\hline 6 & 33 & 666 \\
\hline 8 & 25 & 500 \\
\hline 10 & 20 & 400 \\
\hline
\end{tabular}


In subsurface formations that are suitable for GCS, the hydrostatic pressure near the injection well keeps the $\mathrm{CO}_{2}$ in the liquid or supercritical phase, resulting in the homogeneous fluid-liquid equilibrium (FLE) conditions. The viscosity of the FLE mixtures has been characterized under high pressure and low shear conditions and exhibits an increasing trend with mole fraction of $\mathrm{CO}_{2}$ [Kumagai and Yokoyama, 1999; Bando et al., 2004]. FLE mixtures were found to be Newtonian fluids. As a parcel of $\mathrm{CO}_{2}$ or $\mathrm{CO}_{2}$-saturated brine moves to shallower depths, the pressure of the surrounding formation will decrease and $\mathrm{CO}_{2}$ will undergo a phase transition to create heterogeneous vapor-liquid equilibrium (VLE) mixtures. The literature on rheology of volcanic magma suggests that under certain conditions, the presence of bubbles can either increase or decrease the viscosity of the mixture relative to the continuous phase [Llewellin and Manga, 2005]. The increase is typically associated with streamline distortion as viscoelastic bubbles interfere with fluid flow. An initial input of energy is needed during the evolution of the bubbles from solid-like to liquid-like (elastic to viscous) structures, which results in an apparent shear-thinning behavior. Decreases in mixture viscosity are typically associated with free slip of matrix fluid along the highly deformed bubble surface.

Constitutive relationships of multiphase flow are typically based on the derivation of a relative viscosity term, $\eta_{r}$, which is defined as the ratio of the effective viscosity of the suspension normalized to the suspending matrix fluid. The relative viscosity of a bubbly mixture can generally be expressed as a function of several properties that can be measured in the lab including the volumetric fraction of $\mathrm{CO}_{2(\mathrm{~g})}$ bubbles $(\phi)$, the size distribution of $\mathrm{CO}_{2(\mathrm{~g})}$ bubbles $\left(R_{i}\right.$, the radius of the $i^{\text {th }}$ group of bubbles with identical size), the viscosity of the continuous phase $\left(\mu_{0}\right)$ and capillary number $\left(C_{a}\right)$, a dimensionless number that captures the ratio of viscous shear force to interfacial tension force [Llewellin and Manga, 2005]. 


\subsection{Wettability of Diagenetic Minerals}

The interfacial properties, most notably those between the $\mathrm{CO}_{2}$ and the connate brine, typically measured as interfacial tension, and those between the $\mathrm{CO}_{2}$, brine, and host formation mineral, measured as contact angle $(\theta)$ [Espinoza and Santamarina, 2010], play an crucial role in affecting the underlying physical and chemical processes that drive long-term storage in the subsurface and those that could result in premature leakage [Oldenburg and Lewicki, 2006]. These forces are central for bridging the gap between theoretical predictions and field scale demonstrations of GCS technology.

Two-phase flow through porous media is impacted by the wettability characteristics of the solid phases (e.g., minerals, mineraloids, and organic matter including kerogen or coal) that directly contact pore fluids [Levine et al., 2011]. In formations with heterogeneous surface composition, interfacial forces could also affect flow paths [Juanes et al., 2006]. During leakage events, changes in pressure and temperature, along with phase transitions and mass transfer, will affect contact angles and estimates of transport rates [Oldenburg and Lewicki, 2006]. While the pore surfaces have complex morphology and geometry, contact angle measurements on smooth homogenous surfaces are an effective way to characterize wettability. These measurements are used to model a variety of relevant processes including injectability, sealing capacity, and breakthrough pressure [Buckley, 1996; Morrow et al., 1998]. To date, efforts to understand these processes have generally assumed that geologic repositories are hydrophilic with a constant contact angle [Oldenburg and Lewicki, 2006].

In contrast to interfacial tension, which has been intensively characterized, contact angle measurements have not been developed for many mineral species of interest because of their complex heterogeneity on the pore scale, although it has important effects on a number of pro- 
cesses relevant for understanding GCS [Peters, 2009; Wang and Clarens, 2012]. The existing ones were conducted under limited mineralogy, $\mathrm{pH}$ and salinity conditions [Mills et al., 2011; Espinoza and Santamarina, 2010]. No work has reported on the effects of mass transfer and phase change on wettability. Similarly no work has appeared on the effect of heterogeneous surfaces on transport processes.

Most models of bubble rise through porous media have assumed a constant value for contact angle $\left(150^{\circ}\right)$ [Oldenburg and Lewicki, 2006; Cihan and Corapcioglu, 2008]. In reality this value varies as a function of the composition of the host formation. Accurate models of $\mathrm{CO}_{2}$ bubble rise will not be possible without these measurements. And only with these data, which factors would most strongly influence the wetting characteristics of a host formation can be determined.

Contact angle hysteresis can also have a significant role in influencing $\mathrm{CO}_{2}$ transport and trapping behavior, as the advancing contact angle of the wetting phase displacement is less than the receding contact angle of nonwetting phase displacement due to surface roughness or chemical heterogeneities [Juanes et. al., 2006]. Another source of hysteresis is relative permeability hysteresis. In numerical simulations performed by Juanes and others [2006], the process begins during injection as less wetting $\mathrm{CO}_{2}$ displaces more wetting brine in a drainage-like process, followed by the displacement of $\mathrm{CO}_{2}$ by water at the trailing end of the plume as the $\mathrm{CO}_{2}$ flows upward and laterally due to buoyancy forces. This leads to the formation of $\mathrm{CO}_{2}$ "blobs and ganglia" that become immobile, demonstrating the capillary trapping mechanism [Juanes et. al., 2006]. This trapping of the nonwetting phase of $\mathrm{CO}_{2}$ causes a relative permeability hysteresis, which can then affect subsequent $\mathrm{CO}_{2}$ flow and trapping patterns. Capillary trapping has also 
been demonstrated to be sensitive to changes in injection rate as well as the ratio of gravity to viscous forces. The hysteresis phenomena of contact angle need further investigation.

Recent work has suggested that the contact angle at the $\mathrm{CO}_{2}$-brine-mineral interface is impacted by pressure and temperature and/or the composition of the solid and the connate brine [Espinoza and Santamarina, 2010; Bikkina, 2011; Chiquet et al., 2007a]. Espinoza and Santamarina found a strong correlation between pressure and interfacial tension that manifest in subtle contact angle reductions on calcite and oil-wet quartz [2010]. Chiquet et al. found a similar pressure dependence on mica but not on quartz [2007a]. They attributed this effect to $\mathrm{pH}$ changes in the brine as a function of increased $\mathrm{CO}_{2}$ partial pressure. Like pressure, the effect of temperature on interfacial tension and wettability has been explored in several papers [Bikkina, 2011; Chalbaud et al., 2009]. Temperature effects on contact angle have been found to be subtle and likely related to changes in solubility of $\mathrm{CO}_{2}$ at the higher temperatures. Increases in temperature and pressure are coupled in the subsurface because of geothermal gradients and hydrostatic pressure.

The chemistry of the connate brines (e.g., $\mathrm{pH}$, ionic strength) has also been found to have an important effect on interfacial tension and wettability. Chiquet et al. found that higher salinity rates led to higher contact angles [2007a]. This could be explained by the changes in $\mathrm{CO}_{2}$ solubility with salinity. Temperature, pressure, $\mathrm{pH}$, and salinity also drive equilibrium between the $\mathrm{CO}_{2}$ and brine phases. Several studies have commented on the effect of phase changes (e.g., between supercritical, liquid, and gas phases) on contact angles including Bikkina [2011]. That paper also describes a hysteretic effect associated with repeated exposure of the surfaces to watersaturated $\mathrm{CO}_{2}$. Similarly, Mills et al. explored contact angle hysteresis on a group of relevant minerals using the method of advance-recede cycles and found important differences between these conditions [Mills et al., 2011]. This hysteretic behavior could be driven at least in part by 
contact angle pinning though there is no consensus in the published literature about what causes the hysteresis that has been observed.

Most work to date on wettability in $\mathrm{CO}_{2}$-relavent conditions has been performed for a select subset of mineral surfaces, typically silica/quartz, calcite, or mica [Espinoza and Santamarina, 2010; Chiquet et al., 2007a]. Petroleum engineers have sought to understand how $\mathrm{CO}_{2}$ preferentially binds to coals and other hydrophobic materials relative to natural gas [Sakurovs and Lavrencic, 2011]. Even though these results provide a useful contrast for developing a mechanistic understanding of the behavior of $\mathrm{CO}_{2}$ at the solid/brine interface, these data cannot be directly applied in GCS formations that contain a variety of hydrophilic minerals other than quartz and calcite. Even though sandstones are comprised primarily of quartz, the pore-mineral contact is disproportionately composed of diagenetic minerals that cement the sandstone. Peters found that kaolinite, for example, accounts of roughly 5-31\% of the mineral content in Viking Sandstones but accounted for 65-86\% of pore-mineral contact boundaries [Peters, 2009; Ellis et al., 2011]. This suggests that most of models of bubble rise in the past overestimated contact angle. This work also demonstrated that (1) current understanding of $\mathrm{CO}_{2}$ reactivity in consolidated sandstones is lacking, (2) how surface reaction and complexation models of $\mathrm{CO}_{2}$ in the subsurface might be overestimated once the chemical morphology of these coatings is taken into account. More detailed data on contact angle are needed for developing accurate models.

The complex behavior of the $\mathrm{CO}_{2}$-mineral-brine system, and the buoyancy of $\mathrm{CO}_{2}$ relative to brine, presents methodological challenges for providing reproducible measurements of contact angle in GCS systems. Most of the studies published to date measured contact angles using drops of water on solid surfaces in a $\mathrm{CO}_{2}$ environment. Yang et al. developed a method to measure contact angle using the axisymmetric drop shape analysis technique for a sessile drop of brine in 
a $\mathrm{CO}_{2}$ environment [2008]. This difference between using sessile and pendant drops is important because of the effect of gravity and the hydration layers that exist at the $\mathrm{CO}_{2}$-mineral interface precluding a detailed characterization of solution chemistry. In addition, since much of the wettability literature was developed for petroleum extraction applications, there is a tendency to consider surfaces as either hydrophobic or hydrophilic. Even though $\mathrm{CO}_{2}$ is a nonpolar molecule, with a low dielectric constant, it does have a quadrupole moment that could influence how it interacts with surfaces [Raveendran et al., 2005].

In light of some of the unresolved questions surrounding $\mathrm{CO}_{2}$ wettability under GCS conditions, there were three specific goals for carrying out this work. The first was to understand how brine chemistry, i.e., ionic strength and $\mathrm{pH}$, could have a mineral-specific effect in controlling contact angle. The second was to explore how the temperature and pressure conditions that would prevail during leakage events could impact contact angles. The third and final goal was to study how the contact angles on minerals that are prevalent on the pore surface, primarily clays, which have been largely unstudied, will be influenced by $\mathrm{P}-\mathrm{T}$ conditions.

\subsection{Dispersive (Interfacial) Adhesion}

Interfacial adhesion, also known as dispersive adhesion, has been long observed by researcher in the studies of geological carbon sequestration and enhanced oil recovery that acidic gases such as $\mathrm{CO}_{2}, \mathrm{H}_{2} \mathrm{~S}$ and also non-polar $\mathrm{N}_{2}$. When adhesion happens, $\mathrm{CO}_{2}, \mathrm{H}_{2} \mathrm{~S}$ or $\mathrm{N}_{2}$ strongly bonds to the solid surface and considerable work needs to be to remove the attached bubbles/droplets [Kendall, 1994; Chiquet et al., 2007a; Broseta et al., 2012]. Adhesion mostly accompanies with pronounced contact angle hysteresis and contact line pinning [Buckley et al., 1989]. Therefore, it significantly influences the capillary trapping that is driven by wettabililty. 
Light has been shed on this phenomenon. Both spectroscopic experiments measuring infrared spectra or adsorption isotherm and molecular dynamics simulations suggested that under certain conditions, the nanometer scale water film that originally spreads and wets the surface were disjoined and replaced in the presence of solid-adhesive fluids such as $\mathrm{CO}_{2}$ and $\mathrm{H}_{2} \mathrm{~S}$ [Broseta et al., 2012]. Tripp and Combes [1998] reported that $\mathrm{CO}_{2}$ could penetrate the thin water layer sandwiched between $\mathrm{CO}_{2}$ and the solid and physically adsorbed to the surface.

Adhesion is a macroscopic manifestation of a decline of Gibbs free energy resulted from the complex short range (nanometer scale) van der Waals interactions including molecular affinity, hydrogen bonds, London dispersion forces, etc. The reason is that the adhesive minerals all have hydroxide functional groups, e.g. aluminol ( $(\mathrm{Al}-\mathrm{OH})$ and silanol (=Si-OH) at the solid surface that are sensitive to the adjacent aqueous phase $\mathrm{pH}$ and ionic strength conditions. The molecular affinity can be quantified by calculating the Hamaker constant [Hamaker, 1937]. And the structures of water film can be approximated using Derjaguin-Landau-Versey-Overbeek (DLVO) theory. Combining Hamaker constant and water film thickness, the adhesion force can be estimated using Boer theory [Boer, 1936].

Adhesion is strongly affected by the surface charge distribution, the thickness and stability of the water film and the surface morphology. This phenomenon is further complicated by solution chemistry such as $\mathrm{pH}$, ionic strength and the level of carbonation of brine. It can be either enhanced or diminished by altering $\mathrm{pH}$ and ionic strength. $\mathrm{pH}$ has a determining role in adhesion by controlling the surface charge state. Ionic strength generally enhances adhesion by compressing the electrical double layers (EDL).

Adhesion has significant impacts on reservoir wettability, oil recovery and $\mathrm{CO}_{2}$ storage and thus necessitates a thorough study. Until now, there are limited numbers of solids have been 
discovered showing adhesion. Mechanisms could be further clarified with more experimental data, especially those collected under GCS and EOR related conditions.

\subsection{Modeling Efforts}

Existing work has largely assumed constant values for viscosity and contact angle [Corapcioglu et al., 2004]. Two main schemes, i.e., (1) $\mathrm{CO}_{2}$ bubble free rise in $\mathrm{CO}_{2}$-saturated brine and small $\mathrm{CO}_{2}$ bubble free rise through coarse porous media without contact with pore walls and (2) continuous $\mathrm{CO}_{2}$ channel flow have been modeled separately. Whether these existing models of quantifying $\mathrm{CO}_{2}$ transport in an otherwise brine-saturated porous media are valid under GCS relevant conditions and the relative importance of model parameters including viscosity of matrix fluid, contact angle between $\mathrm{CO}_{2}$ and formation minerals, $\mathrm{CO}_{2}$ bubble size, porosity, grain size, pore size of porous media, tortuosity, permeability and saturation need to be investigated.

The experimental data collected in this work on the rheological, wetting and adhesive phenomena are particularly valuable to be integrated to evaluate the leakage behavior in the context of buoyancy-driven flow through porous media collectively. These data should be incorporated into the models such as TOUGH2 and efforts should be directed to upscale the pore scale phenomena to quantifiable reservoir scale estimation coupling complex interactions between geophysics, geochemistry, geothermal, geometry, geomechanics and fluid mechanics.

\subsection{Summary}

Leakage is inherently challenging to study because $\mathrm{CO}_{2}$, driven by buoyant forces, travels over long distances, undergoing phase changes and encountering numerous connate brine and 
formation chemistries as it rises to the surface. Thorough understanding of leakage on both the pore scale and on the reservoir scale requires more efforts to be undertaken in several key areas. Important gaps in current state of knowledge on $\mathrm{CO}_{2}$ leakage during GCS have been identified:

1 - Experimental data and constitutive relationships are lacking on the rheological behavior, especially under the VLE conditions where most pronounced leakage ensues. The effects of pressure, temperature and ionic strength on the viscosity of $\mathrm{CO}_{2}$-bine mixtures have not been clarified;

2 - Reported experimental data are insufficient that wettability of very limited number of mineral species in the $\mathrm{CO}_{2}$-brine environment has been studied and the data are inconsistent. The influence of geochemistry including $\mathrm{pH}$, ionic strength, pressure and temperature on wettability was not explored;

3 - Interfacial adhesion phenomena which provide robust trapping to $\mathrm{CO}_{2}$ has rarely been reported and considered when estimating the overall trapping capacity of a particular reservoir. Rise velocity of $\mathrm{CO}_{2}$ could be significantly overestimated and the trapping capacity could be seriously underestimated without acknowledging the influence of adhesion. Also, the favorable conditions to cause adhesion and the effects of geochemistry and mineralogy on adhesion were not elucidated;

4 - Existing modeling frameworks on the two-phase flow of $\mathrm{CO}_{2}$-brine through porous media are inadequate. Models need to be modified and validated with updated experimental data to account for multiple effects associated with the flow properties of multiphase mixtures, adhesion, wettability and permeability hysteresis, pore geometries, $\mathrm{pH}$ and ionic strength, flow instability and figuring, etc. 
The goal of this work is to collect experimental data on important geophysical and geochemical parameters such as viscosity, interfacial tension and contact angle and its hysteresis collected under GCS related conditions are of fundamental importance to accurately evaluate the leakage potential on the reservoir scale and thus facilitate better site selection and more effective strategies of leakage prevention. This work strives to resolve the above-listed questions and bridge the gaps in experimental data and modeling frameworks. 


\section{CHAPTER 3}

\section{Rheology of $\mathrm{CO}_{2}$-Brine Mixtures}

The effects that $\mathrm{CO}_{2}$ has on the rheological properties of brine solutions over a range of GCS-relevant temperature, pressure, ionic strength, and shear conditions are explored here. Under the fluid-liquid equilibrium (FLE) conditions that prevail in the deep subsurface, viscosity of $\mathrm{CO}_{2}$-brine mixtures was found to be a function of temperature and pressure alone. Once leakage conditions ensue, discrete $\mathrm{CO}_{2}$ bubbles form in brine resulting in the vapor-liquid equilibrium (VLE) and these mixtures exhibit complex linear viscoelastic, time dependent and thixotropic behavior. The presence of $\mathrm{CO}_{2(\mathrm{~g})}$ bubbles on the flow of the bulk fluid could have important impacts on impeding (via shear drag force) leakage depending on the geometrical, geochemical and geophysical characteristics of a storage site. Under VLE conditions, the apparent viscosity of $\mathrm{CO}_{2}$-brine mixtures was found to be up to five times higher than brine alone but the microstructure was easily destroyed, and not readily regained, under high shear conditions. At higher temperatures and higher ionic strength, the effect is less pronounced. These results were considered in the context of flow through porous media and the effect on buoyancy-driven flow is significant. Understanding this effect is important for developing an accurate constitutive relationship for leaking $\mathrm{CO}_{2}$, which will lead to better capacity to select and monitor GCS sites. 


\subsection{Experimental Methods}

The rheological properties of $\mathrm{CO}_{2}$-brine mixtures were characterized using an AntonPaar $^{\circledR}$ Physica MCR 301 rotational rheometer. The rheometer allowed for the measurement of viscosity values over a broad range of temperature, pressure and shear conditions. The rheometer was equipped with two interchangeable high-pressure cells rated up to $15 \mathrm{MPa}$ with precise $\left( \pm 0.1^{\circ} \mathrm{C}\right)$ peltier-style temperature control. The two different size cells were used to measure low $\left(<100 \mathrm{~s}^{-1}\right)$ and high $\left(>100 \mathrm{~s}^{-1}\right)$ shear rates. Both cells were equipped with double-gap measuring geometries especially designed for low viscosity measurements. Since the effective shear measured by the rheometer is perpendicular to the circumference of the measuring tool, but not to the fluid, the shear force can be decomposed into a normal force and an axial extensional force [Djéridi et al., 1999]. This effect has been reported for study of bubbly flow in the past and is especially relevant for understanding how bubbles will flow through porous media where bubbles will experience shear and extensional forces from the pore walls and pore throats [Nakken et al., 2001; Thompson et al., 2001]. In addition, the gap in the measurement tool is on the same order of magnitude of the pores that would exist in sequestration sites to capture scale-dependent effect on bubble deformation. The $\mathrm{CO}_{2}$-brine mixtures were created using a Teledyne ISCO ${ }^{\circledR}$ 500HP high-pressure syringe pump with a constant temperature jacket. The $\mathrm{CO}_{2}$ was delivered as received (Roberts Oxygen Co., Charlottesville, VA). The brine was made using analytical grade sodium chloride (Fisher Scientific, Hanover Park, IL) and was used as delivered. The pressure cell was also attached to a custom-built high-pressure view cell to enable real-time recording and visualization of the phase behavior of the $\mathrm{CO}_{2}$-brine mixture using a $\mathrm{CCD}$ firewire camera. A schematic of the experimental setup is presented in Figure 3.1. 

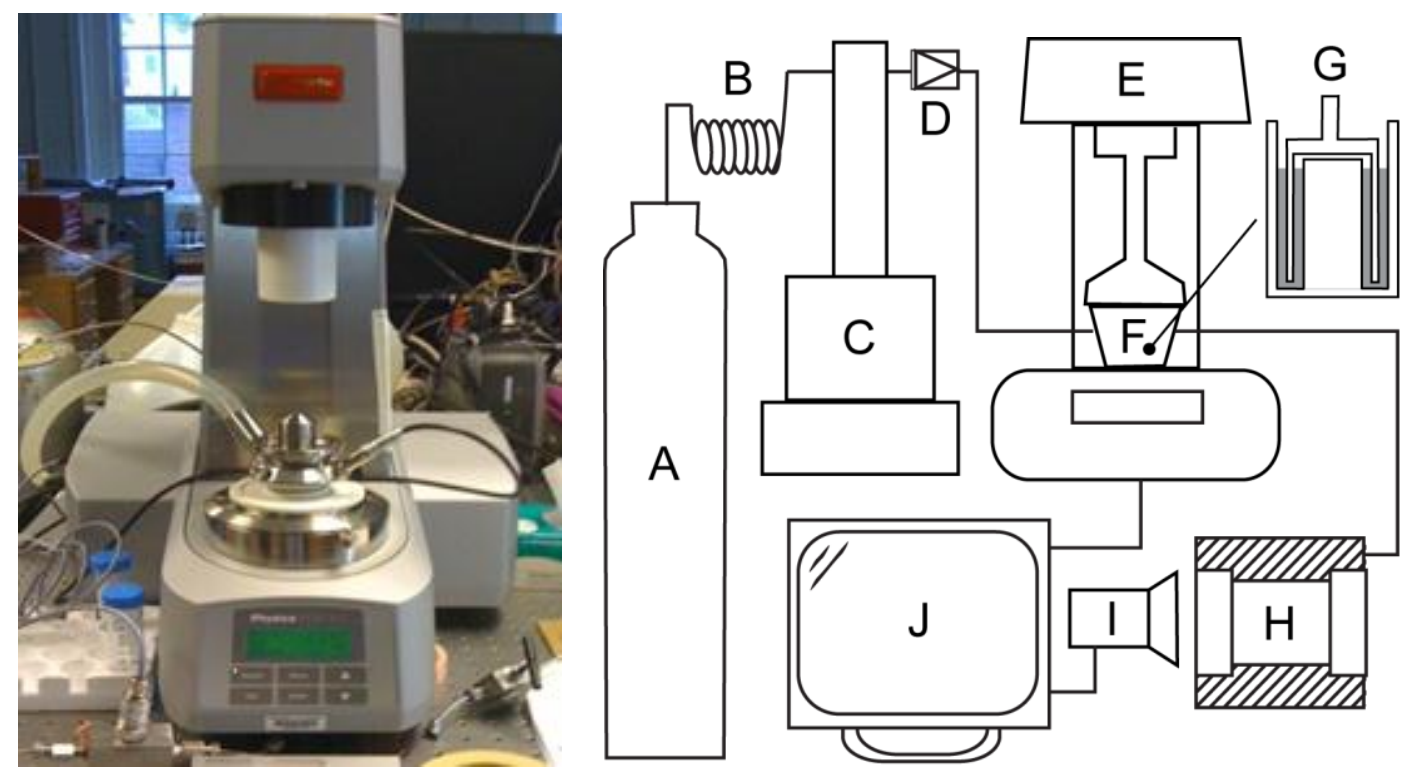

Figure 3.1. Schematics of experimental setup. A. tank of food-grade $\mathrm{CO}_{2}$, B. heat exchanger, C. syringe pump, D. check valve, E. rotational rheometer, F. high pressure measuring cell, G. double gap attachment, H. high-pressure view cell, I. digital camera, J. computer.

The experimental setup employed here was found to produce accurate results for shear rates ranging from 40 to $1000 \mathrm{~s}^{-1}$. The transition between laminar and turbulent flow was found to occur around a shear rate of $1100 \mathrm{~s}^{-1}$ by experimental trials and calculations based on published protocol [Nakken et al., 2001]. No measurements were made above this value. To eliminate the possibility that dissolved air could interfere with the measurements, the water sample was boiled prior to use in the experiment. In addition, the components of the pressure cell were pre-wetted and allowed to equilibrate with the sample to eliminate interference from entrained air. All samples were given adequate time, usually on the order of several hours, to reach equilibrium of saturation before running an experiment. The exact composition of the $\mathrm{CO}_{2}$-brine solutions was calculated using the method described by Bando et al. [Bando et al., 2003] and using the solubility relationship shown in Figure 3.2. Under both fluid liquid equilibrium (FLE) and 
vapor liquid equilibrium (VLE) conditions (Figure 3.3), these curves were used to identify both the composition of the mixture and the pressure at which phase separation would occur.

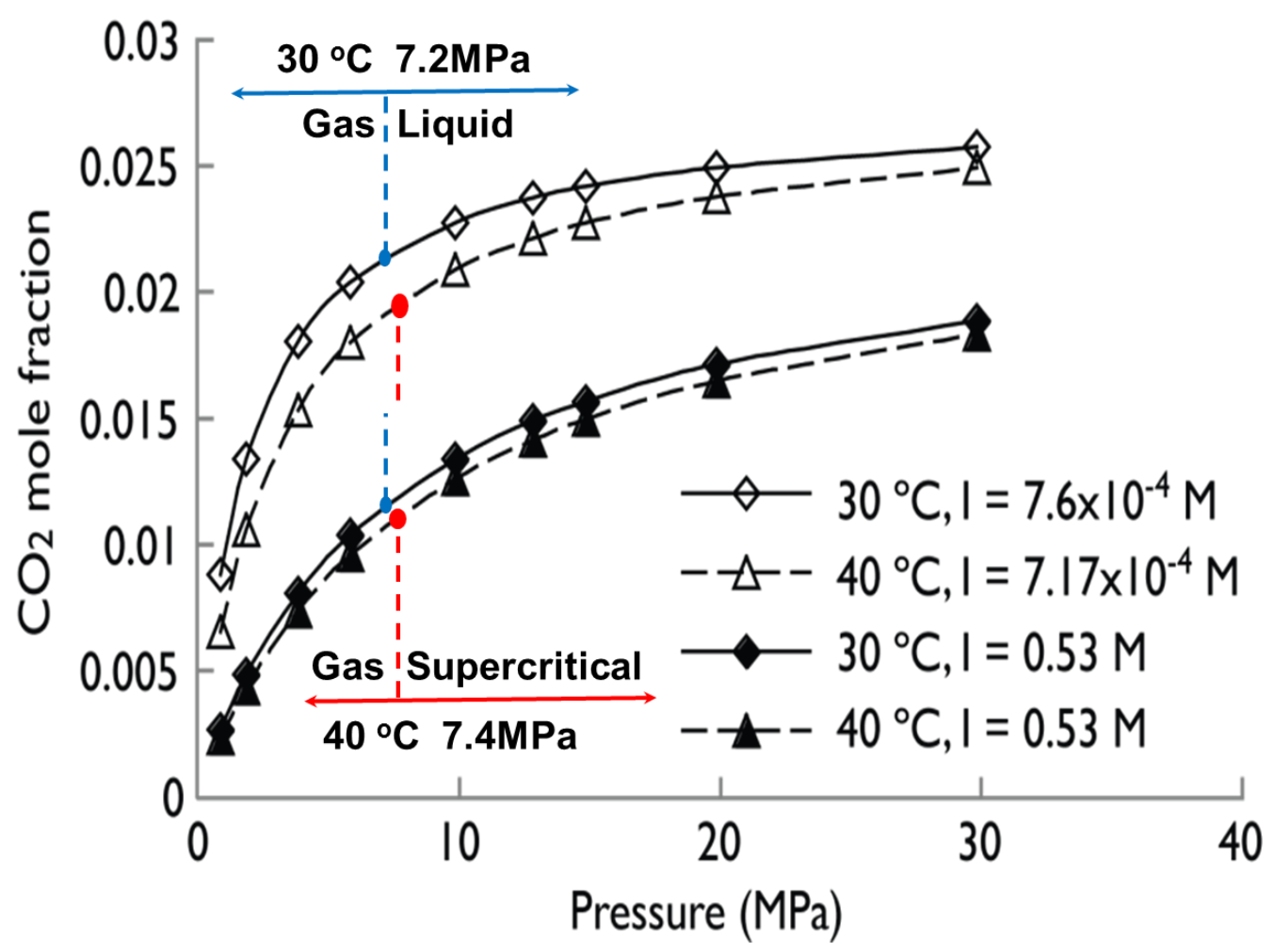

Figure 3.2. Solubility of $\mathrm{CO}_{2}$-brine mixtures was controlled to produce conditions that were representative for a rising bubble of $\mathrm{CO}_{2}$ along its path toward the ground surface. All of the results in this study were conducted for pressures at or below $10 \mathrm{MPa}$, since this is the range most relevant for leakage processes and the least characterized in the existing literature. All experimental methods were also benchmarked using deionized water to ensure that the methods produced the expected behavior and to produce a comparison with the $\mathrm{CO}_{2}$-brine systems.

The mixtures under FLE equilibrium were made by pressurizing the high-pressure vessel in the rheometer and adding $\mathrm{CO}_{2}$ to achieve the desired conditions. To create VLE conditions, saturated $\mathrm{CO}_{2}$-brine mixtures under FLE conditions were gradually depressurized to create mul- 
tiple-phased $\mathrm{CO}_{2}$ bubbly suspension. Figure 3.2 illustrates how a small change in pressure can elicit a significant change in solution chemistry particularly at the low-pressure ranges that were the focus of this work. Figure 3.3 provides a visual example of the distinction in phase behavior between FLE and VLE conditions in the context of $\mathrm{CO}_{2}$-brine flow through the subsurface. Upon depressurization, liquid or supercritical $\mathrm{CO}_{2}$ will maintain a distinct meniscus with water that minimizes interfacial area as seen in Figure 3.3a. As the $\mathrm{CO}_{2}$ transitions to gas phase, bubbles will form as seen in Figure 3.3b. This creation of an immiscible new phase can be understood in terms of the solubility of $\mathrm{CO}_{2}$ in water and the thermodynamics that govern the formation of this new phase. As seen in Figure 3.2, the derivative of the solubility of $\mathrm{CO}_{2}$ in water is much greater at lower pressures. A fixed drop in pressure will result in much more phase separation when in the gas phase versus the liquid or supercritical phase [Oldenburg and Lewicki, 2006]. The accretion of $\mathrm{CO}_{2}$ is unfavorable for the liquid or supercritical $\mathrm{CO}_{2}$ by virtue of their lower interfacial tension with brine and greater energy barrier. During the experiments, a stir bar was placed inside the view chamber and the shear conditions were set to match those in the rheometer pressure cell. With the stir bar spinning, reorientation, rearrangement and deformation of bubbles did occur. Under the experimental shear conditions, no significant inter-bubble interactions such as collision or nucleation of bubbles were observed so the modeling efforts focus on bubble-liquid interactions and neglect bubble-bubble interactions. Within the shear rate range, no wall slip of $\mathrm{CO}_{2}$ bubbles on the inner surface of the pressure view cell was observed. In addition, no significant normal stress difference or stress relaxation was detected. 


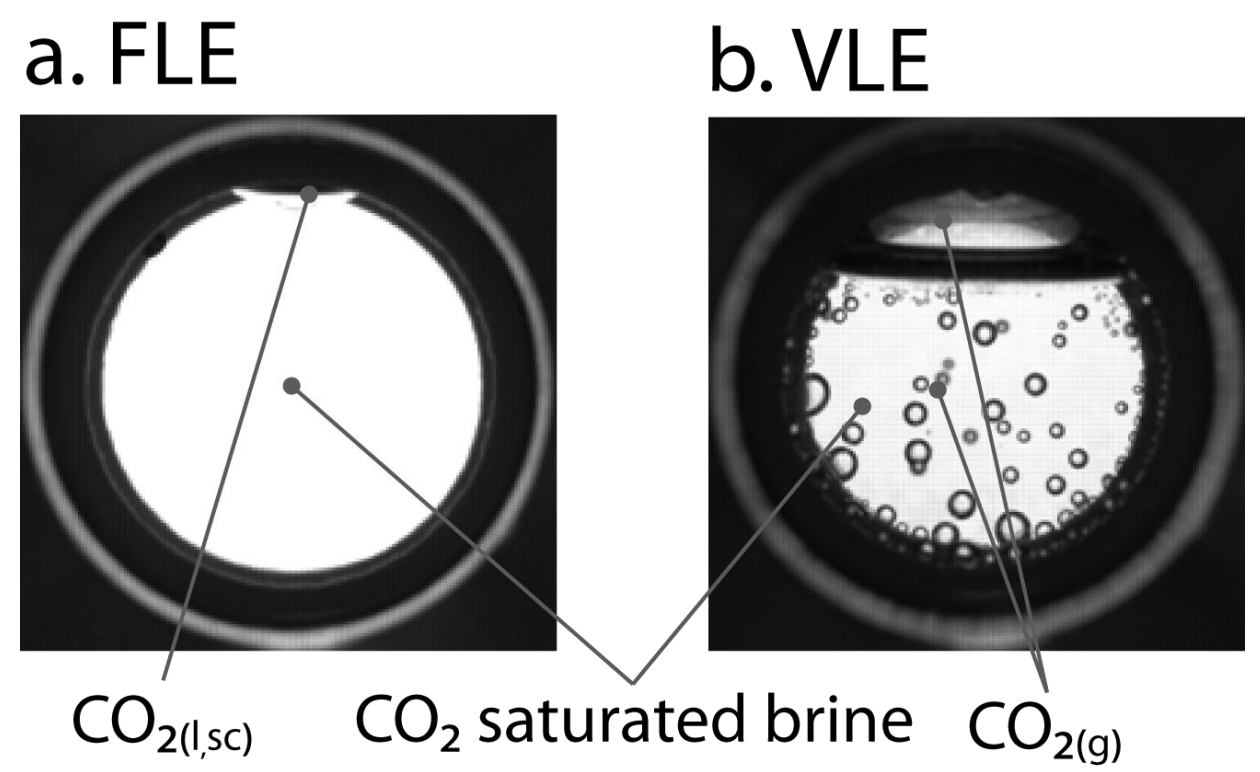

Figure 3.3. The experimental work presented here focused on a. $\mathrm{CO}_{2}$-saturated brines under FLE conditions and b. $\mathrm{CO}_{2}$-brine suspension under VLE conditions. Here, images of these two conditions are provided as seen through the high-pressure view cell connected to the rheometer.

\subsection{Rheology of $\mathrm{FLE} \mathrm{CO}_{2}$-brine Mixtures}

In subsurface formations that are suitable for GCS, the hydrostatic pressure near the injection well keeps the $\mathrm{CO}_{2}$ in the liquid or supercritical phase. Under the resulting FLE conditions, the $\mathrm{CO}_{2}$-brine mixtures exhibit Newtonian behavior and the presence of $\mathrm{CO}_{2(\mathrm{aq})}$ increases the viscosity of mixture as shown in Figure 3.4. The magnitude of the viscosity increase is generally proportional to the $\mathrm{CO}_{2(\mathrm{aq})}$ concentration in the solution. This effect has been reported before [Bando et al., 2004; Kumagai and Yokoyama, 1999] and is consistent with the behavior of other acid solutions [Laliberte, 2007]. The results presented in Figure 3.4 are for a lower pressure or $\mathrm{CO}_{2}$ mole fraction $\left(\chi_{\mathrm{CO}_{2}}\right)$ range than has appeared in the literature to date. Since $\mathrm{CO}_{2}$ mole fraction is controlled by the partial pressure of $\mathrm{CO}_{2}$ in equilibrium with the aqueous phase, the $x$ axis in Figure 3.4 could be expressed using the relationship in Figure 3.2. This range was select- 
ed specifically to enable better understanding of leakage processes from geologic sequestration sites. Interestingly, the results reveal a subtle dip in the values near the pressure that $\mathrm{CO}_{2}$ transitions from liquid/supercritical fluid to gas (around a $\mathrm{CO}_{2}$ mole fraction of 0.01). This effect can be understood in light of two competing mechanisms that drive the viscosity of the FLE mixture. The first is associated with the acidification of the brine solution resulting from the dissolution of $\mathrm{CO}_{2}$ in the mixture and the formation of carbonic acid, represented by the top line in Figure 3.5. Aqueous acid solutions have higher viscosity than water on account of the more intimate molecular interactions between conjugate species [Kumar et al., 2008; Laliberte, 2007; ZafaraniMoattar and Majdan-Cegincara, 2009]. The second effect produces a decrease in the viscosity of the brine as a result of mixing a lower viscosity fluid, in this case $\mathrm{CO}_{2}$ with the brine, represented by the bottom group of lines in Figure 3.5. The latter effect is less significant so that the net effect of $\mathrm{CO}_{2}$ on the brine is to increase the viscosity, though the importance of both mechanisms is evident near the vapor/liquid transition point. 


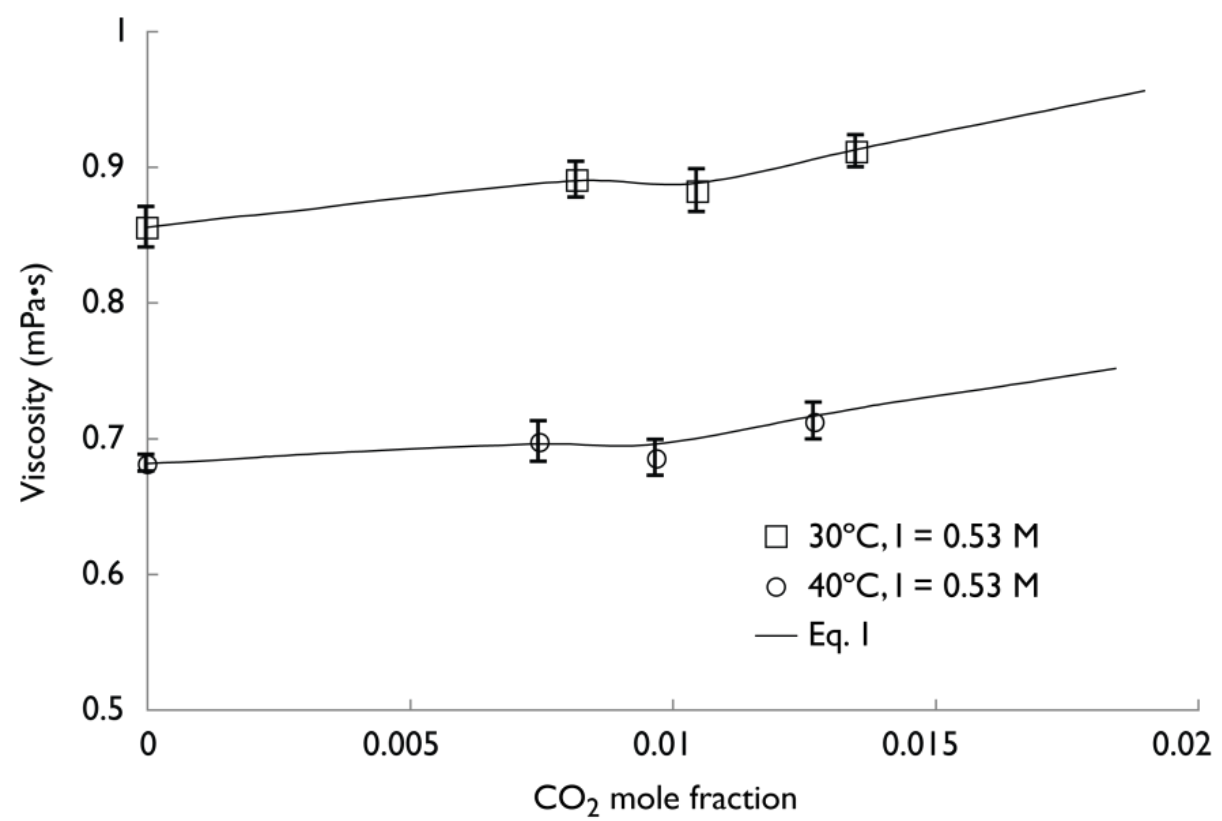

Figure 3.4. The viscosity of $\mathrm{CO}_{2}$-brine mixtures under FLE conditions increases with $\mathrm{CO}_{2}$ concentration as shown in other work, but the results here, for a lower equilibrium concentration of $\mathrm{CO}_{2}$ show a dip around the point that $\mathrm{CO}_{2}$ transitions from liquid $\left(30{ }^{\circ} \mathrm{C}\right) /$ supercritical $\left(40{ }^{\circ} \mathrm{C}\right)$ to gas. The four data points on each line correspond to experimental conditions of $0.1,4,6$ and $10 \mathrm{MPa}$ from left to right. These data are for $\mathrm{I}=0.53 \mathrm{M}$ but the same trend was observed at lower ionic strength values. Error bars represent $90 \%$ confidence interval.

The competing mechanisms that influence the viscosity of $\mathrm{CO}_{2}$-brine mixtures under FLE conditions can be understood in the context of Arrhenius type mixing rules. This type of model suggests that the viscosity of a binary mixture will be proportional to the relative composition (in this case $\chi_{b r i n e}$ and $\left.\chi_{\mathrm{CO}_{2}}\right)$ and the viscosity $\left(\mu_{b r i n e}\right.$ and $\mu_{\mathrm{CO}_{2}}$ ) of those two fluids. The GrunbergNissan equation, which is based on Arrhenius-type behavior but also includes an interaction parameter for the species in solution, accurately predicts the behavior of these mixtures [Kijevčanin, 2008; Laliberte, 2007; Soli and Byrne, 2002; Tomida et al., 2007]. In this case, the 
interaction term accounts for the increase in viscosity that is associated with adding a weak acid to the solution:

$$
\ln \mu_{m, F L E}=\chi_{\mathrm{CO}_{2}} \ln \mu_{\mathrm{CO}_{2}}+\chi_{\text {brine }} \ln \mu_{\text {brine }}+\frac{1}{2} \chi_{\mathrm{CO}_{2}} \chi_{\text {brine }}\left(G_{\mathrm{CO}_{2}-\text { brine }}+G_{\text {brine }-\mathrm{CO}_{2}}\right)
$$

where $\mu_{m, F L E}$ is the viscosity of the mixture under FLE conditions, $\mu_{i}$ and $x_{i}$ are the viscosity and the mole fraction of species i respectively. $G$ is the interaction coefficient of components. In the case of $\mathrm{FLE} \mathrm{CO}_{2}$-brine homogenous mixtures, the Grunberg-Nissan equation does a good job of approximating the experimental results of Figure 3.4. In particular, at the transition from liquid/supercritical to gaseous $\mathrm{CO}_{2}$, the $\mu_{\mathrm{CO}_{2}}$ changes substantially. The net effect on the viscosity of the solution is a dip near the transition pressure. Experimental data of pure substances including brine and $\mathrm{CO}_{2}$ were used and a graphical representation of this effect at $30{ }^{\circ} \mathrm{C}$ is provided in Figure 3.5. The top line provides an estimate of how the viscosity of a $\mathrm{FLE} \mathrm{CO}_{2}$-brine mixture should look according to the Grunberg-Nissan equation if carbonic acid only resulted in producing a higher viscosity solution like other acids. The bottom grouping of lines shows how the viscosity of a $\mathrm{CO}_{2}$-brine solution might be interpreted if $\mathrm{CO}_{2}$ only had the effect of reducing the viscosity of the mixture, since the viscosity of the $\mathrm{CO}_{2}$ is always lower than the brine. Several lines are used since the properties of $\mathrm{CO}_{2}$ change as a function of equilibrium pressure and that is proportional to the composition plotted on the $x$ axis. For a range of $\chi_{\mathrm{CO}_{2}}$, the model accurately captures the viscosity behavior of the mixture, including the dip in viscosity near the pressure at which $\mathrm{CO}_{2}$ transitions from liquid/supercritical to gas phase. 


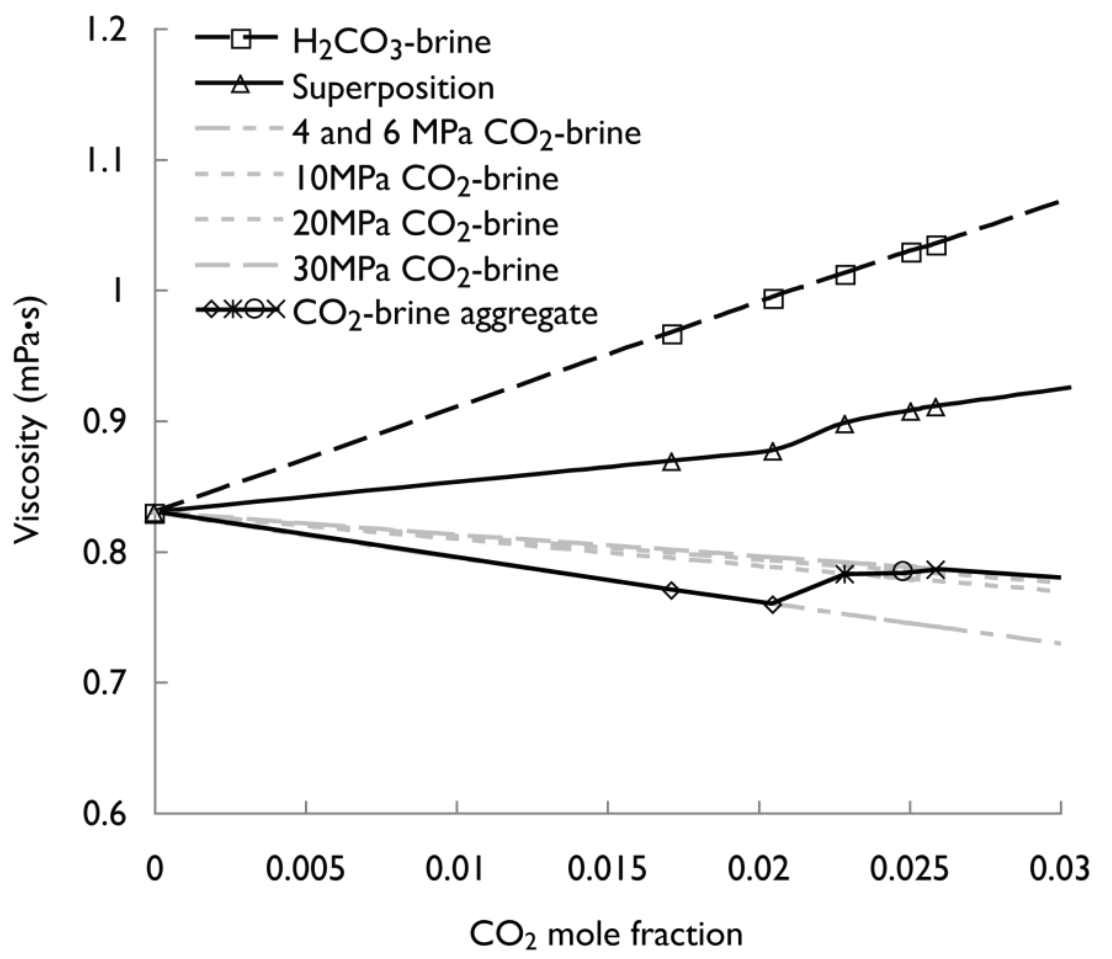

Figure 3.5. The Grunberg-Nissan model accurately captures the viscosity of FLE mixtures. The viscosity of $\mathrm{CO}_{2}$-brine solutions generally increases with $\mathrm{CO}_{2}$ concentration even though there is a minor drop in viscosity near the point where $\mathrm{CO}_{2}$ transitions from liquid/supercritical to gas.

In the broader context of predicting leakage from geologic sequestration sites, the dip in solution viscosity observed under the FLE condition will likely have only a small effect on bubble rise. The effect that temperature and ionic strength play in driving the viscosity of the brine solutions, both in terms of the viscosity of the water and in limiting the solubility of $\mathrm{CO}_{2}$, is much more pronounced than the dip that occurs near the transition from liquid/supercritical to gas phase $\mathrm{CO}_{2}$. Additional tests, i.e., shear curve, transient, and hysteretic tests, reveal that FLE mixtures are Newtonian under variable shear conditions or over time, with the shear curve results shown in Figure 3.6. This is expected since both brine and $\mathrm{CO}_{2(\mathrm{~g}, 1, \mathrm{sc})}$ are Newtonian fluids. The 
results are highly consistent with published data for low shear conditions providing a useful benchmark for the experimental techniques used here [Bando et al., 2004].

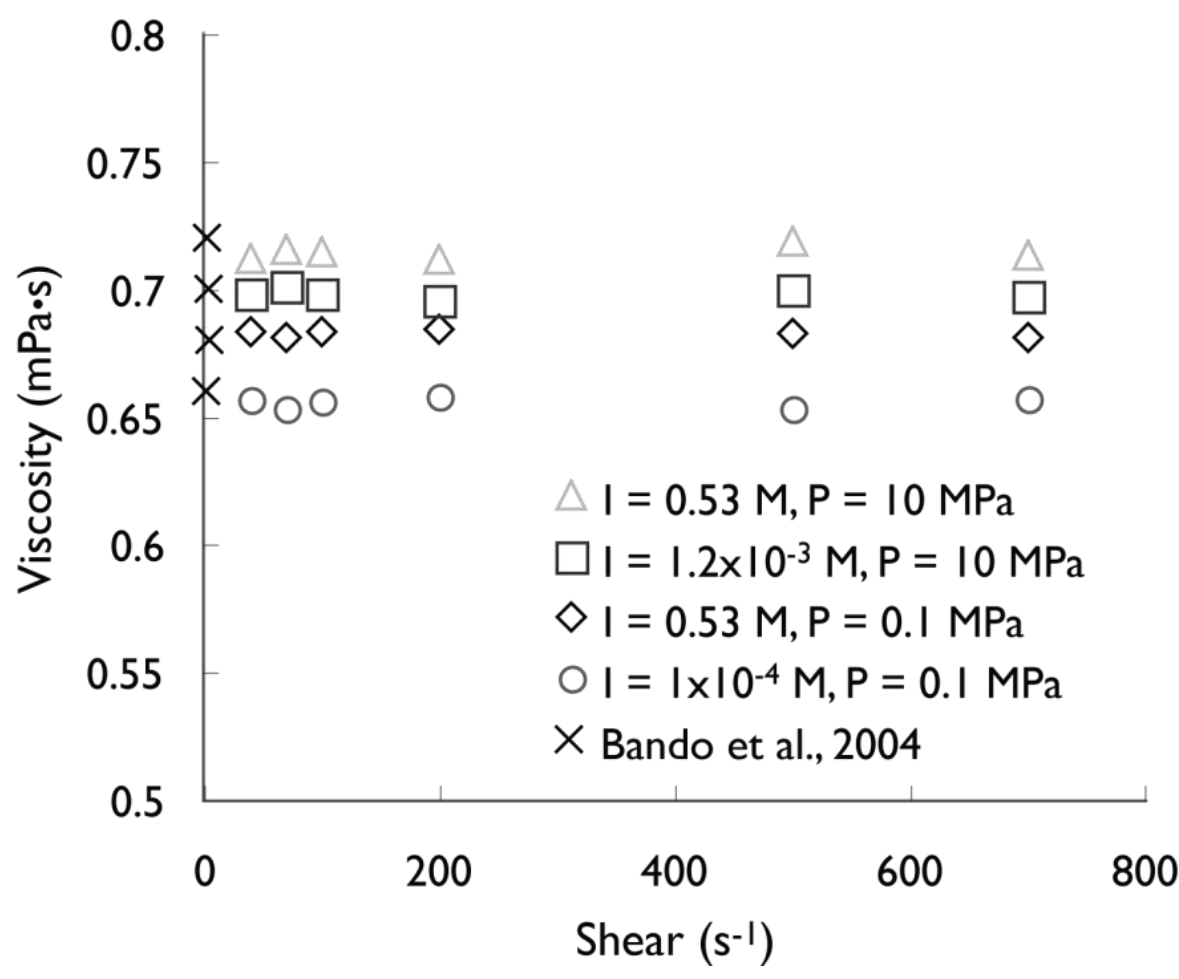

Figure 3.6. Under FLE conditions, $\mathrm{CO}_{2}$-brine solutions exhibit Newtonian behavior over a wide range of shear rates. The data presented here were collected at $40^{\circ} \mathrm{C}$ but the trend is consistent at $30{ }^{\circ} \mathrm{C}$.

\subsection{Rheology of VLE $\mathrm{CO}_{2}$-brine Mixtures}

As $\mathrm{CO}_{2}$ or $\mathrm{CO}_{2}$-saturated brine moves to shallower depths, the pressure of the surrounding formation will decrease and $\mathrm{CO}_{2}$ will undergo a phase transition to create vapor-liquid equilibrium (VLE) conditions. The results of experiments under VLE conditions were found to be somewhat more complex because of the presence of viscoelastic $\mathrm{CO}_{2}$ bubbles. Extensive work in 
volcanology has demonstrated how magma flows with entrained gas bubbles exhibit nonNewtonian flow behavior [Manga and Loewenberg, 2001]. This behavior is driven indirectly by mixture composition and state variables like temperature, and pressure and more directly by bubble volume and size distribution, shear rate, and time [Llewellin and Manga, 2005]. There are differences between magma and brine in terms of viscosity, interaction with gases, and shear conditions that require separate analysis. A series of shear sweep, transient and thixotropic tests was designed to understand the effect that $\mathrm{CO}_{2}$ bubbles will have on brine flows under VLE conditions.

Studies of the VLE flow conditions in the $\mathrm{CO}_{2}$-brine system confirmed that there was a nonlinear relationship between shear stress and shear rate. The results of Figure 3.7 demonstrate that under lower pressure conditions $(\leq 4 \mathrm{MPa})$ where VLE occurs, the mixture exhibits time dependent behavior. The effective viscosity of the mixture is initially quite high but the viscosity decreases readily under applied shear toward a steady state that is higher than the viscosity of the brine alone. After tens of seconds under applied shear, the effective viscosity of the solution approaches the steady-state value. To explain this, it is helpful to envision how a single $\mathrm{CO}_{2(\mathrm{~g})}$ bubble might resist deformation at the beginning of the experiment while its shape is changed from spherical to elliptical. The viscoelastic behavior that is observed in the bulk fluid is the result of many bubbles of different sizes exhibiting resistance to applied shear stress and the corresponding increase in the gas-liquid interfacial area and energy per unit volume [Klempner and Frisch, 1991]. Steady-state conditions are characterized by equilibrium between the interfacial tension force and shear stress. Interestingly, the steady-state viscosity of the mixture is always higher than that of the FLE conditions. This is significant because it suggests that even over long time periods, the presence of $\mathrm{CO}_{2(\mathrm{~g})}$ bubbles could suppress leakage relative to brine with $\mathrm{CO}_{2(\mathrm{aq})}$ dis- 
solved in it. The results of Figure 3.7 also suggest that at lower shear rates, the steady state viscosity of the VLE mixture is higher. This is because at lower shear, the shearing energy acting to deform the bubbles is lower and so the streamline deformation effect caused by $\mathrm{CO}_{2(\mathrm{~g})}$ in the solution is more pronounced causing a higher effective viscosity for the mixture. In other words, the larger the shear forces placed on the bubbly $\mathrm{CO}_{2}$-brine suspension, the closer it approximates the effective viscosity of a $\mathrm{CO}_{2}$-brine solution under comparable FLE conditions.

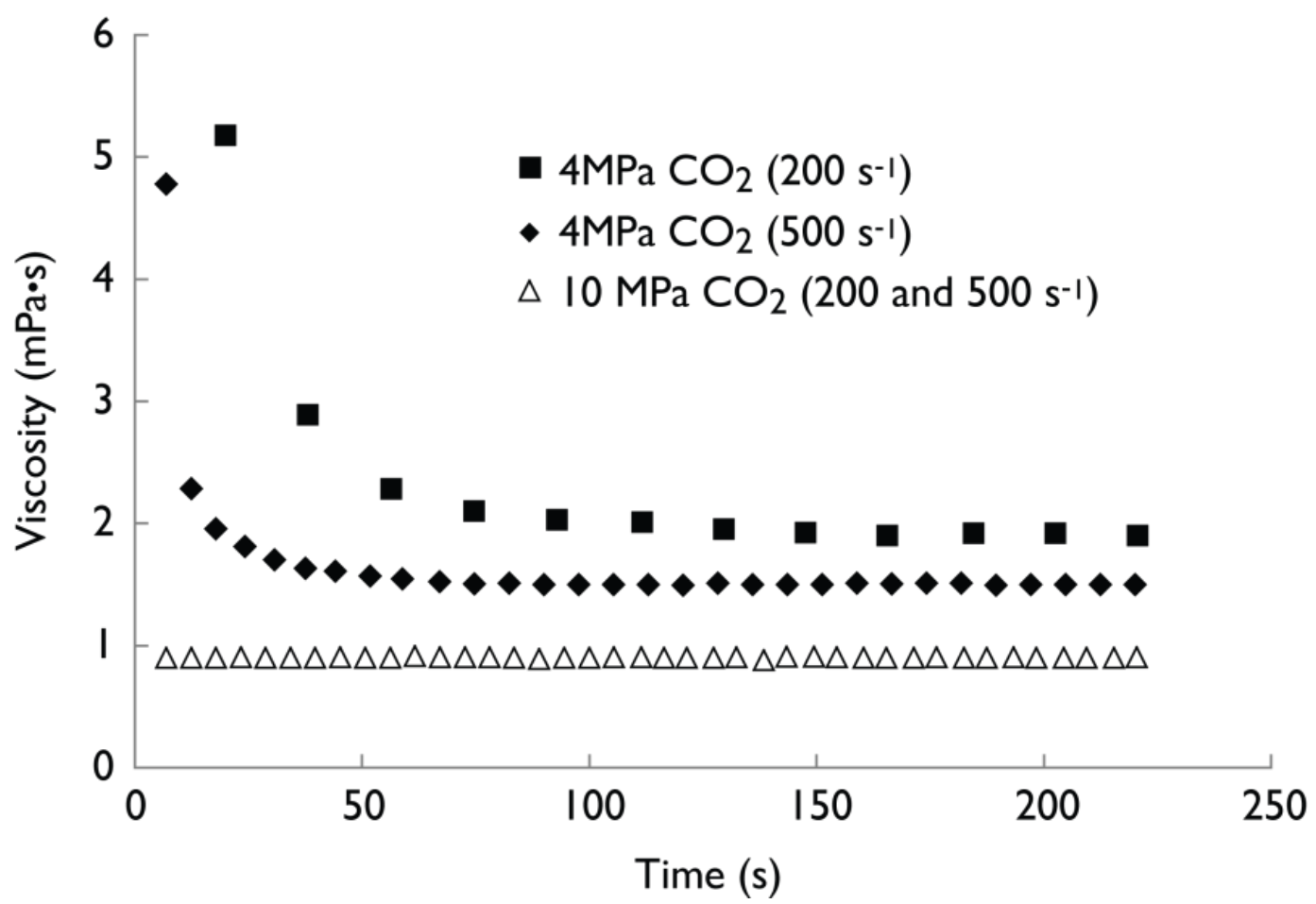

Figure 3.7. The viscosity of $\mathrm{CO}_{2}$-brine mixtures under VLE conditions at $30^{\circ} \mathrm{C}, \mathrm{I}=0.53 \mathrm{M}$ and two shear rates (shaded markers) reveals that complex flow behavior can be expected once $\mathrm{CO}_{2(\mathrm{~g})}$ bubbles appear in the brine resulting in different steady-state effective viscosities for the mixture. FLE results are represented using markers with no fill. 
The effect of entrained bubbles on fluid flow has been explored in great detail for a number of other systems [Llewellin et al., 2002a]. The literature suggests that under certain conditions, the presence of bubbles can either increase or decrease the viscosity of the mixture relative to the continuous phase. The increase is typically associated with streamline distortion as viscoelastic bubbles interfere with fluid flow. An initial input of energy is needed during the evolution of the bubbles from solid-like to liquid-like (elastic to viscous) structures, which results in an apparent shear-thinning behavior as seen in Figure 3.7. Decreases in mixture viscosity are typically associated with free slip of matrix fluid along the highly deformed bubble surface. Even though preliminary experiments suggested that $\mathrm{CO}_{2}$-brine mixtures would typically fall into the former category, it was worth exploring the exact conditions that led to this behavior.

Constitutive relationships of multiphase flow are typically based on the derivation of a relative viscosity term, $\eta_{r}$, which is defined as the ratio of the viscosity of the suspension normalized to the suspending matrix fluid. The relative viscosity of a bubbly mixture can generally be expressed as a function of several properties that can be measured in the lab including the volumetric fraction of $\mathrm{CO}_{2(\mathrm{~g})}$ bubbles $(\phi)$, the size distribution of $\mathrm{CO}_{2(\mathrm{~g})}$ bubbles $\left(R_{i}\right.$, the radius of the $\mathrm{i}^{\text {th }}$ group of bubbles with identical size), the viscosity of the continuous phase $\left(\mu_{0}\right)$ and capillary number $\left(C_{a}\right)$, a dimensionless number that captures the ratio of viscous shear force to interfacial tension force [Llewellin and Manga, 2005] given by:

$$
C_{a}=t_{r} \dot{\gamma} \quad \text { Eq. } 3.2
$$

where $\dot{\gamma}$ is the shear rate, and $t_{r}$ is the bubble relaxation time, which is a measure of the timescale over which a bubble can respond to changes in its shear environment [Llewellin and Manga, 
2005]. The $t_{r}$ is a weakly increasing function of $\phi$ and is expressed as $t_{r}=\frac{\mu_{0} a}{\sigma}$ where $a$ is the radius of the undeformed $\mathrm{CO}_{2(\mathrm{~g})}$ bubble and $\sigma$ is the interfacial tension between $\mathrm{CO}_{2(\mathrm{~g})}$ bubble and brine [Llewellin and Manga, 2005]. For unsteady flows, where the rate of change of the shear strain rate, $\ddot{\gamma}$, must be considered, it is common to use the dynamic capillary number, given by:

$$
C_{d}=t_{r} \frac{\ddot{\gamma}}{\dot{\gamma}} \quad \text { Eq. } 3.3
$$

For the $\mathrm{CO}_{2}$-brine mixtures considered here, $t_{r}$ was found to be $3.3 \times 10^{-6} \sim 1 \times 10^{-4} \mathrm{~s}$ using published interfacial tension values [Bachu and Brant Bennion, 2009]. $\phi$ and $R_{i}$ were 0.5 4 vol\% and $2 \times 10^{-4} \sim 5 \times 10^{-3} \mathrm{~m}$ respectively. $C_{a}$ was used for the steady-state experiments (i.e., Figures 3.7-3.9) and $C_{d}$ for the unsteady flow experiments (i.e., Figure 3.10 and 3.11 ) and were found to be $6.7 \times 10^{-4} \sim 1 \times 10^{-1}$ and $8.3 \times 10^{-8} \sim 5 \times 10^{-3}$, respectively.

A number of semi-empirical relationships between $C_{a}$ or $C_{d}, \phi$, and $\eta_{r}$, have been proposed in previous work [Llewellin et al., 2002a; Murai and Oiwa, 2008; Sochi, 2010]. Several equations were tested here and the best fit was obtained using a model that accounts for polydispersity and instability of bubbles called the gamma probability density function given by:

$$
\eta_{r}=\left(1-\frac{5}{3} \phi\right)+\frac{8}{3} \phi \int_{0}^{\infty} \frac{\frac{\beta^{\alpha}}{\Gamma(\alpha)} R_{i}^{\alpha-1} e^{-\beta R_{i}}}{1+\frac{9}{16}\left(\frac{\mu_{0} \dot{\gamma}}{\sigma}\right)^{2}} R_{i}^{2} \quad \text { Eq. } 3.4
$$

where $\Gamma(\alpha)$ is the gamma distribution function, $\mu_{0}$ is the viscosity of the continuous phase, and $\alpha$ and $\beta$ are the shape and scale parameters respectively, which are obtained by fitting experimental results [Joh et al., 2010]. This model provides accurate approximations of the rheology of 
bubbly flow for polydispersed bubble suspensions in shear flows over a wide range of capillary numbers [Joh et al., 2010; Sang-Yoon et al., 1997].

The relative viscosity of $\mathrm{CO}_{2}$-brine mixtures under VLE conditions at steady state is presented in Figure 3.8. Both experimental and modeling results based on Equation 3.4 are presented. The capillary number on the $x$ axis in Figure 3.8 is calculated based on the weight-averaged $R_{i}$. The results are consistent with the data presented in Figure 3.7, where the effective viscosity of the bubbly suspension is higher than the viscosity of the $\mathrm{CO}_{2}$-saturated brine alone (i.e., $\left.\eta_{r}>1\right)$. It was determined that $\mathrm{CO}_{2}$-brine suspensions will always increase the viscosity of the solution and this can be explained using two characteristics of bubbly flow. The first is that brines have a relatively low viscosity and so the capillary number will always be $<<1$. As described above, $C_{a}$ was several orders of magnitude lower than 1 and per bubbly flow rheology, this is the transition point at which streamline deformation becomes less pronounced and shear slip along the bubble brine interface becomes the dominant characteristic of the fluid. The second reason that $\mathrm{CO}_{2}$ bubbles will always increase the viscosity of brines in geologic carbon sequestration conditions is that the flow of interest occurs through porous media and the capillary number in these types of conditions is characteristically low. 


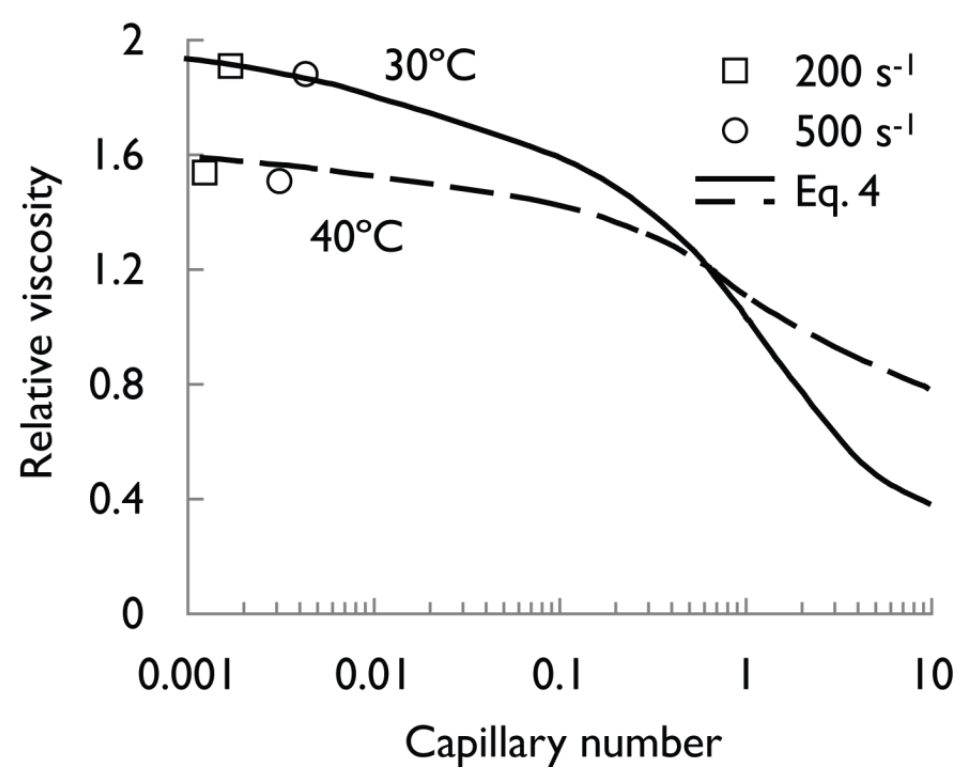

Figure 3.8. The steady-state relative viscosity of VLE $\mathrm{CO}_{2}$-brine suspensions will always be higher than the viscosity of the suspending matrix fluid, pure brine saturated with $\mathrm{CO}_{2}$. This has important implications for multiphase flow in leakage situations related to carbon sequestration. Experimental results presented here are at $2 \mathrm{MPa}$ and $\mathrm{I}=0.53 \mathrm{M}$.

It is important to note that most of the work on relative viscosity to date assumes that the liquid/supercritical phase and the gas are immiscible. This is not the case for $\mathrm{CO}_{2}$ in brine since the two are quite miscible and under non-equilibrium conditions, applied shear could accelerate gas exchange with the brine since it would increase the contact area between the two phases. The results of this effort could help inform models in which the $\mathrm{CO}_{2(\mathrm{~g})}$ is leaking into brine-filled formations for the first time (e.g., in terms of predicting mixing rates and so on) but these were not the conditions assessed here. This work was carried out for the case where the brine is already saturated with $\mathrm{CO}_{2}$ under equilibrium conditions. Geologic sequestration sites must be stable over decades and centuries and so equilibrium conditions are of the greatest interest.

To evaluate the effect that solution chemistry has on the complex flow of $\mathrm{CO}_{2}$-brine suspensions under VLE conditions, the rheology of the system was measured under two ionic 
strength conditions, $\sim 0$ and $0.53 \mathrm{M}$. The results, presented in Figure $3.9 \mathrm{a}$ and $3.9 \mathrm{~b}$ respectively, suggest that ionic strength has a net effect of decreasing the effective viscosity of the suspension. This is because the presence of salts in the solution suppresses the formation of $\mathrm{CO}_{2}$ bubbles. Interestingly, ionic strength had the opposite effect on effective viscosity under FLE conditions because the presence of salts in the brine lowered the solubility of $\mathrm{CO}_{2}$ in solution as shown in Figure 3.2 and 3.6. From a practical perspective, this suggests that the complex flow behavior can be expected deeper in the subsurface in less saline formations. These results also highlight the strong temperature dependence that is observed in these systems. Since fewer $\mathrm{CO}_{2}$ bubbles form at higher temperatures, the result is a lower effective viscosity for the suspension. The importance of ionic strength is less pronounced at higher temperatures. For both temperatures, the VLE viscosity is substantially higher than it would be under FLE conditions for the same pressure, i.e., comparing the results of Figures 3.4, 3.6, and 3.9. 

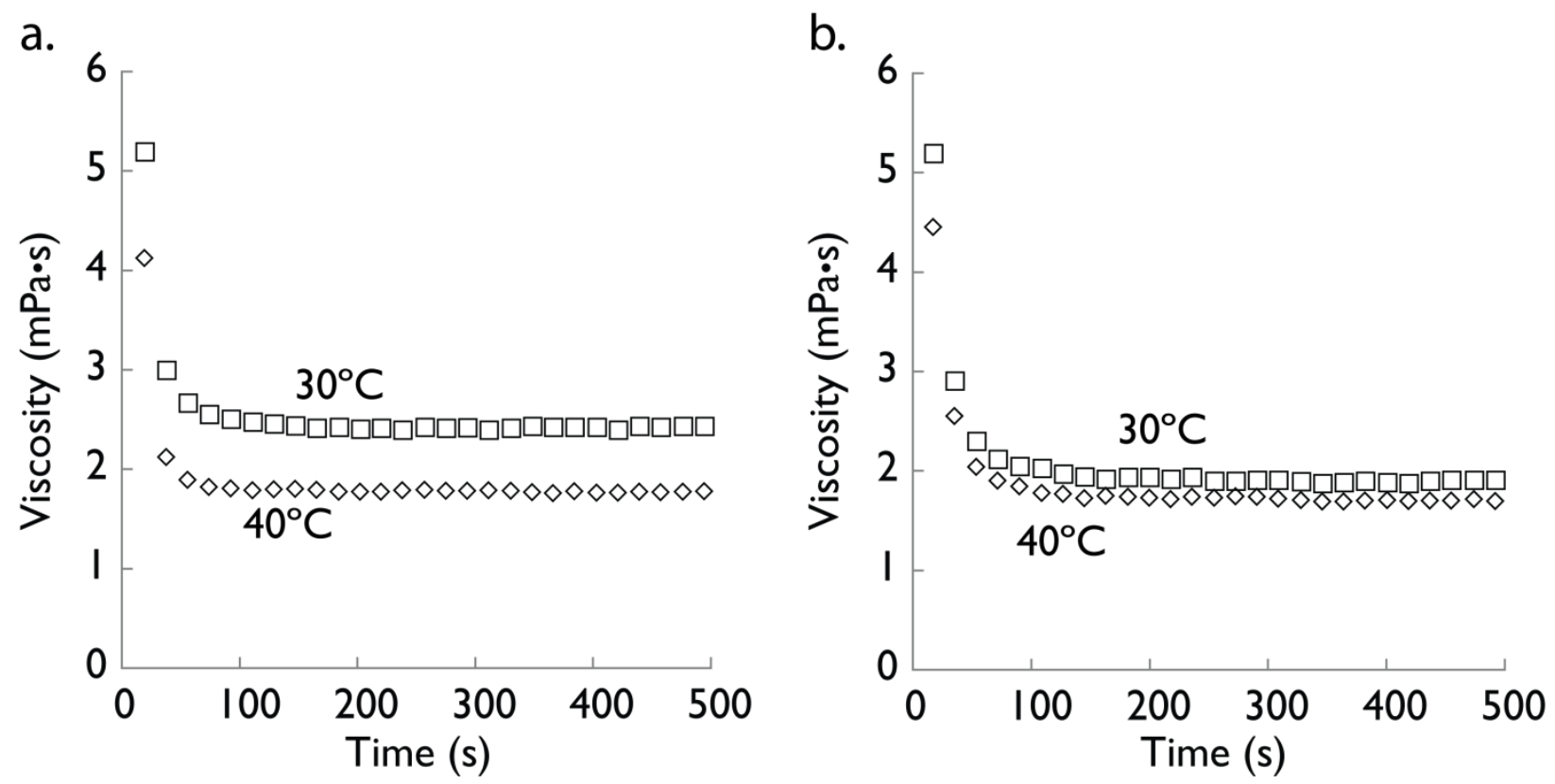

Figure 3.9. The viscosity of $\mathrm{CO}_{2}$-brine mixtures under VLE conditions (4 MPa at shear rate of $\left.200 \mathrm{~s}^{-1}\right)$ at two temperatures $\left(30^{\circ} \mathrm{C}\right.$ and $\left.40^{\circ} \mathrm{C}\right)$ and ionic strength $\left(\mathrm{a} . \mathrm{I}=7.17 \sim 7.6 \times 10^{-4} \mathrm{M}, \mathrm{b} . \mathrm{I}=\right.$ $0.53 \mathrm{M})$ conditions. Higher temperature and ionic strength both suppress the formation of $\mathrm{CO}_{2(\mathrm{~g})}$ bubbles, which result in lower values of steady-state viscosity.

Another important characteristic of the $\mathrm{CO}_{2}$-brine mixtures under VLE conditions is that the viscosity of the original mixture is not readily regained once the shear forces are removed. This makes sense intuitively because $\mathrm{CO}_{2(\mathrm{~g})}$ bubbles will become distorted or destroyed in the solution under the applied shear. To measure this behavior in the laboratory, a series of experiments was performed in which the shear on a sample was steadily increased from $200 \sim 1000 \mathrm{~s}^{-1}$ followed immediately by a decrease in the shear rate back to $200 \mathrm{~s}^{-1}$. Both the increase and the decrease occurred at the same rate. No rest period was allowed between the two tests. The general trend of these shear sweep tests is illustrated in Figure 3.10. It is clear that as the shear rate increases, there is a significant drop in viscosity, but that as shear rate is decreased, the mixture 
viscosity only recuperates to a fraction of its original value. These two curves constitute a socalled hysteresis loop, which is an important characteristic of viscoelastic mixtures. This behavior is most often observed in gels and other complex fluids where hydrogen or ionic bonds result in microstructuring of the fluid [Barnes, 1997]. No yield stress was present under the conditions tested. Here the complex behavior is caused solely by the presence of two phases. As the structural units, $\mathrm{CO}_{2(\mathrm{~g})}$ bubbles in this case, are deformed, there is elongation, rearrangement, and breakdown of bubbles from larger to smaller size and from polydisperse to an increasingly monodisperse distribution.

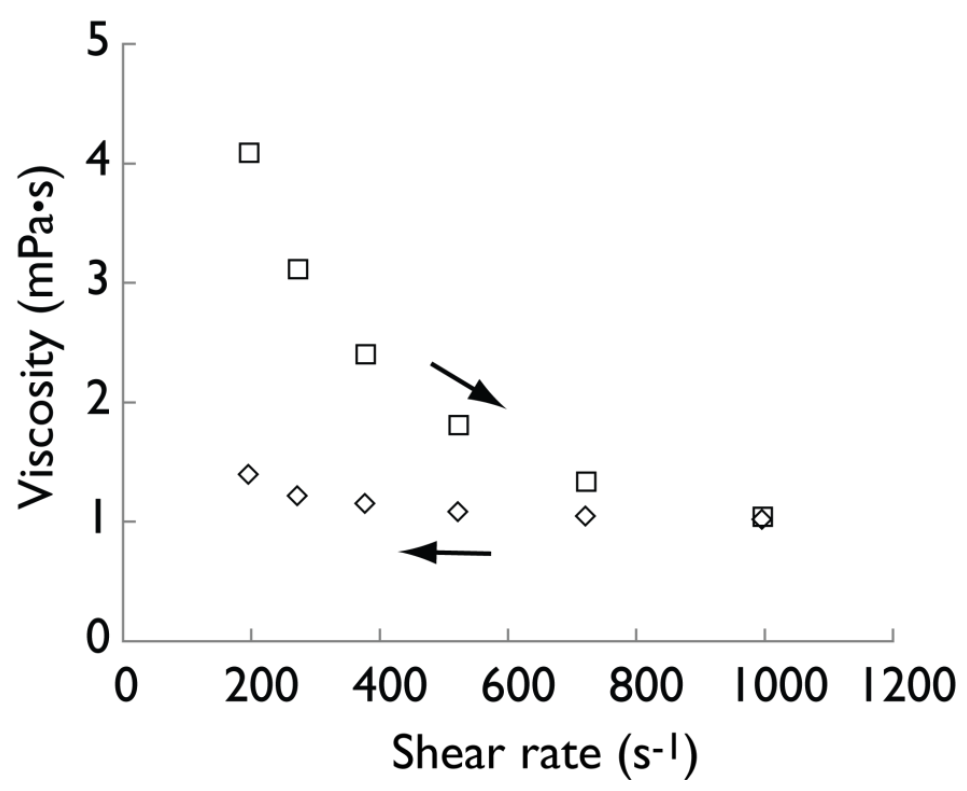

Figure 3.10. Under VLE conditions, $\mathrm{CO}_{2}$-brine mixtures exhibit flow hysteresis whereby the presence of $\mathrm{CO}_{2(\mathrm{~g})}$ bubbles results in a drop in viscosity as shear is applied to the fluid. This structure is lost permanently as the shear is removed from the fluid. This path dependence has important implications for site selection in GCS (these results are for $30^{\circ} \mathrm{C}, \mathrm{I}-0.53 \mathrm{M}$ ).

Consistent with the other experiments reported here, the path dependence of the $\mathrm{CO}_{2^{-}}$ brine suspensions when subjected to shear forces is strongly dependent on the pressure, temperature, and ionic strength of the mixture. The shape, height, and area of the hysteresis-loop in Fig- 
ures 3.10 and 3.11 is proportional to the period and rate of change of shear along with the past kinematic history of the suspension [Chhabra et al., 2008]. The area is related to the energy that is needed to break down the thixotropic structure of the suspension. The size and number of bubbles in the different solutions is a function of ionic strength and the state variables and so it follows that the energy embodied in the mixtures is different as shown in Figure 3.11. Temperature was found to have the most significant impact as shown in Figure 3.11a and 3.11b as well as in Figure 3.11c and 3.11d. The effect of ionic strength was more modest as seen in Figure 3.11a and 3.11c as well as in Figure 3.11b and 3.11d. This behavior was tested for several pressures and the trends were highly consistent for all pressures where VLE conditions exist. 

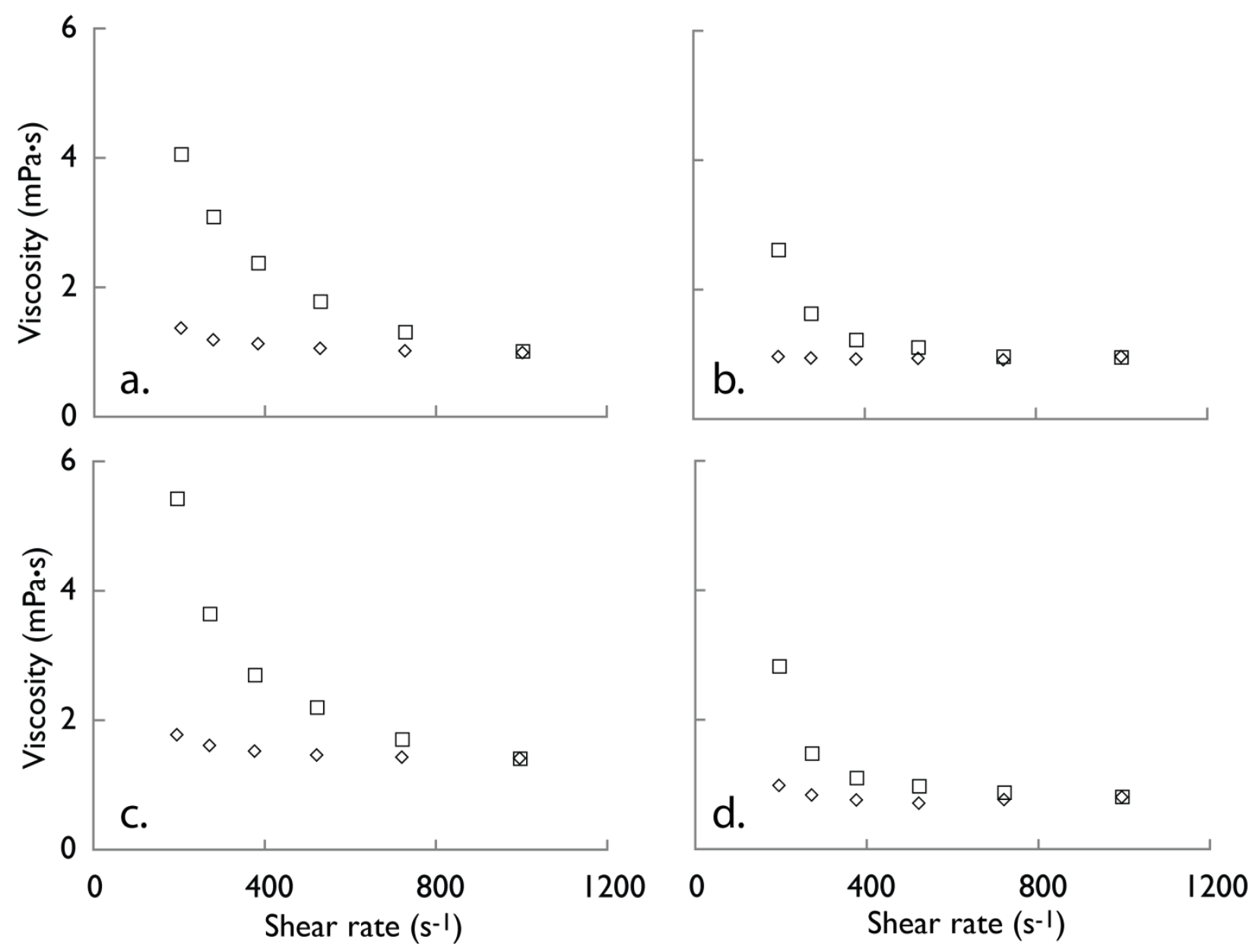

Figure 3.11. Both temperature and ionic strength reduce the magnitude of the thixotropic behavior that was observed under VLE conditions. (a) $30^{\circ} \mathrm{C}, \mathrm{I}=0.53 \mathrm{M}$; (b) $40^{\circ} \mathrm{C}, \mathrm{I}=0.53 \mathrm{M}$; (c) $30^{\circ} \mathrm{C}, \mathrm{I}=3.1 \times 10^{-4} \mathrm{M} ;(\mathrm{d}) 40^{\circ} \mathrm{C}, \mathrm{I}=3.7 \times 10^{-4} \mathrm{M}$. All experiments were conducted at $1 \mathrm{MPa}$. 


\subsection{Effects on Flow through Porous Media}

The results of these experiments will impact bubbly flow of $\mathrm{CO}_{2}$ through porous media in general by slowing the rate of leakage. As leaked $\mathrm{CO}_{2}$-saturated brine moves closer to the surface, the solubility will drop resulting in the ebullition of small $\mathrm{CO}_{2}$ bubbles. These newly formed bubbles will migrate upwards and expand as pressure declines along the leakage path. It is important to reiterate that this analysis is for the VLE case in which $\mathrm{CO}_{2}$ exists in the gas phase. The surface tension between $\mathrm{CO}_{2}$ and brine or water under high-pressure conditions is too low to support the propagation of small bubbles [Hebach et al., 2002]. At lower pressure (e.g., < $5 \mathrm{MPa}$ ), the interfacial tension is large enough that we could reasonably assume that small bubbles would form based on the Young-Laplace equation and the Kelvin equation [Adamson, 1997]. The pressure inside the $\mathrm{CO}_{2}$ bubble, described by Henry's Law must still exceed the sum of the hydrostatic pressure and the surface tension between $\mathrm{CO}_{2}$ and brine $[$ Crandell et al., 2010].

When a $\mathrm{CO}_{2}$ bubble is rising freely without contacting the pore wall $\left(R_{\mathrm{CO}_{2}}<R_{P}\right)$, vertical transport is driven solely by buoyant forces and retarded by drag forces (Figure 2.1). For low Reynold's number conditions, the rise velocity of the $\mathrm{CO}_{2}$ bubble can be modeled using Stokes' law [Poletto and Joseph., 1995]:

$$
u=\frac{d_{C O_{2}}^{2} g\left(\rho_{\text {matrix }}-\rho_{C O_{2}}\right)}{\gamma \mu_{\text {matrix }}}
$$

where $u$ is the rise velocity of $\mathrm{CO}_{2}$ bubble, $d_{\mathrm{CO}_{2}}$ is the diameter of $\mathrm{CO}_{2}$ bubble, $\rho_{\text {matrix }}$ and $\rho_{\mathrm{CO}_{2}}$ are the density of the matrix fluid and the $\mathrm{CO}_{2}$ respectively, $\mu_{\text {matrix }}$ is viscosity of the matrix fluid and $\gamma$ is a viscosity coefficient expressed as $\gamma=12 \frac{\mu_{\text {matrix }}+1.5 \mu_{\mathrm{CO}_{2}}}{\mu_{\text {matrix }}+\mu_{\mathrm{CO}_{2}}}$. Since $\mu_{\text {matrix }}>>\mu_{\mathrm{CO}_{2}}, \gamma=12$ 
. The Stokes' law is appropriate for small bubbles only because (1) small $\mathrm{CO}_{2}$ bubbles in brine ensure that the Reynold's number is low $\left(R_{e}=\frac{\rho_{\text {matrix }} u d_{\mathrm{CO}_{2}}}{\mu_{\text {matrix }}} \sim 1\right)$ and (2) because small $\mathrm{CO}_{2}$ bubbles can be treated as rigid bodies with spherical geometry [McGinnis et al., 2006].

Using the fluid viscosity $\left(\mu_{\text {matrix }}\right)$ results extracted from Figures 3.4, 3.6, 3.7, 3.8, 3.9, 3.10 and 3.11 (summarized in Table 3.1), the effects on the Stokes' law estimates of bubble rise velocity can be assessed. The results, presented in Figure 3.12, demonstrate that both matrix viscosity and $\mathrm{CO}_{2}$ bubble size affect the rise velocity according to a quadratic proportionality. The viscosity of the brine has an important effect on controlling terminal rise velocity over a range of bubble sizes even though the effect is most pronounced for larger bubbles. This result is worth consideration in light of the viscosity data presented in this work. Once bubble ebullition commences, terminal velocity could drop by more than $75 \%$ over a range of relevant bubble sizes. Larger bubbles have a higher terminal velocity, e.g., the terminal velocity of a $0.00075 \mathrm{~m}$ bubble is 100 times that of $0.000075 \mathrm{~m}$ bubble. The net effect of viscosity and $\mathrm{CO}_{2}$ bubble size on terminal velocity depends on the ratio $\frac{\left(\Delta d_{\mathrm{CO}_{2}}\right)^{2}}{\Delta \mu_{\text {matrix }}}$. As an example, if a $\mathrm{CO}_{2}$ bubble follows the P-T path described in pathway a in Figure 2.2, it will have a rise velocity in the range of 0.00085 to $0.643 \mathrm{~m} / \mathrm{s}$ depending on the salinity of the endogenous brine. These swings in the rise velocity over orders of magnitude are significant for beginning to bound the uncertainty associated with estimates of $\mathrm{CO}_{2}$ leakage from GCS sites. 
Table 3.1. Experimental data of viscosity $(\mathrm{mPa} \cdot \mathrm{s})$ used in Stokes Law

\begin{tabular}{|c|c|c|c|c|c|}
\hline & & \multicolumn{2}{|c|}{$\begin{array}{r}\text { Vapor-Liquid Equilib- } \\
\text { rium (VLE) }\end{array}$} & \multicolumn{2}{|c|}{$\begin{array}{r}\text { Liquid-Liquid } \\
\text { Equilibrium } \\
\text { (LLE) }\end{array}$} \\
\hline & & \multicolumn{4}{|c|}{ Ionic strength of brine (M) } \\
\hline $\begin{array}{r}\text { Temperature } \\
\qquad\left({ }^{\circ} \mathrm{C}\right)\end{array}$ & $\begin{array}{r}\text { Pressure } \\
\text { (MPa) }\end{array}$ & $\begin{array}{r}3.1- \\
7.6 \times 10^{-4}\end{array}$ & 0.53 & $\begin{array}{r}3.1- \\
7.6 \times 10^{-4}\end{array}$ & 0.53 \\
\hline 30 & 1 & $\begin{array}{l}1.43 \sim 5.39 \\
2.37 \sim 5.42\end{array}$ & $\begin{array}{l}0.98 \sim 4.14 \\
1.98 \sim 5.37\end{array}$ & $\begin{array}{l}0.84 \\
0.86\end{array}$ & $\begin{array}{l}0.85 \\
0.88\end{array}$ \\
\hline 40 & 1 & $\begin{array}{l}0.85 \sim 3.85 \\
1.85 \sim 4.18\end{array}$ & $\begin{array}{l}0.96 \sim 3.63 \\
1.66 \sim 4.46\end{array}$ & $\begin{array}{l}0.65 \\
0.67\end{array}$ & $\begin{array}{l}0.66 \\
0.69\end{array}$ \\
\hline
\end{tabular}

a.

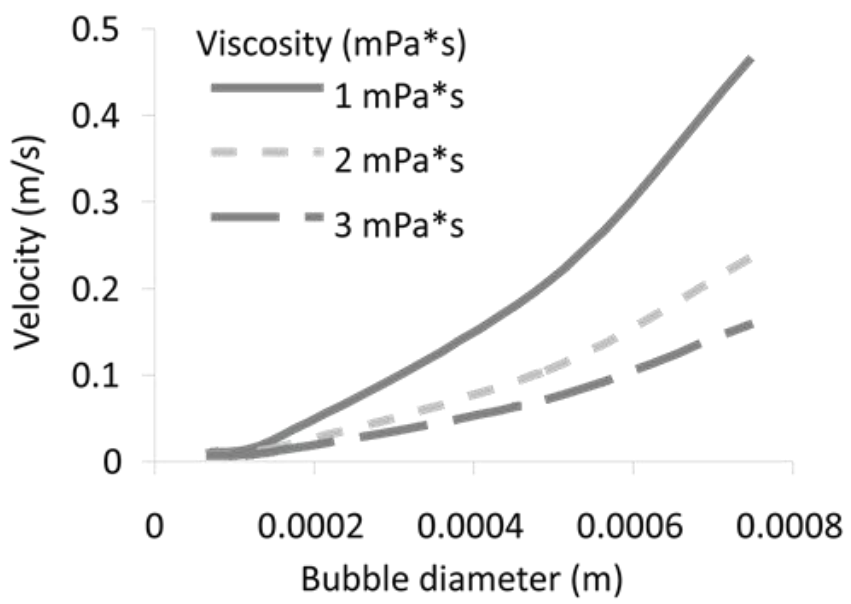

b.

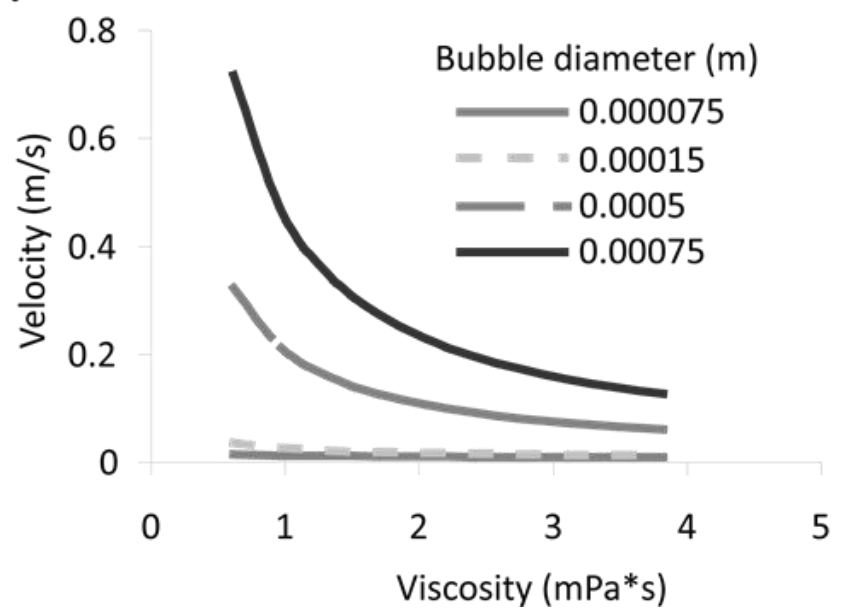

Figure 3.12. Based on Stokes' Law, (a) bubble size and (b) viscosity of the endogenous fluid has a dramatic effect on controlling the rise velocity of buoyancy driven flow of $\mathrm{CO}_{2}$ through brines. 
For the case in which a $\mathrm{CO}_{2}$ bubble is in contact with the pore walls as channel flow ( $R_{\mathrm{CO}_{2}} \geq R_{P}$ ) or as polydisperse bubbly flow, the rising bubble will experience surface tension force that comes from the ternary contact between the brine, solid, and $\mathrm{CO}_{2}$ in addition to the buoyant and drag forces experienced by a free bubble. Most of the $\mathrm{CO}_{2}$ will flow under this scenario and so it is of the most interest for understanding how flow through porous media will proceed under these so called channel flow conditions. The modeling framework used to understand this flow scenario is based on the work of Corapcioglu et al. [Corapcioglu et al., 2004]. The framework is based on a macroscopic force balance and several assumptions: (1) the brine and the $\mathrm{CO}_{2}$ are treated as incompressible; (2) the endogenous brine is saturated with $\mathrm{CO}_{2}$ such that mass transfer between the bubble and the bulk is not considered; (3) the $\mathrm{CO}_{2}$ is in instantaneous equilibrium with respect to temperature and pressure; (4) the porous media is isotropic and fully saturated with brine before $\mathrm{CO}_{2}$ penetration; (5) there is no bubble generation mechanisms such as snap-off and division; (6) there is no Basset history force that results from the viscous effects generated by the acceleration of a particle relative to a fluid under the creeping flow conditions [Zhang and Fan, 2003]; (7) the lift force acting on a bubble is neglected because of irrotational flow conditions [Soubiran and Sherwood, 2000]; (8) the fluid flow in the endogenous brine around a rising bubble and within the fluid bubble is neglected; and (9) velocity gradients are only considered in the vertical direction. This modeling framework has been shown to be a good predictor of bubble rise in unconsolidated media [Corapcioglu et al., 2004].

The governing equation incorporates inertial force, added mass force, buoyant force, surface tension force and drag force that results from the momentum transfer between different phases, shown in Figure 2.1 [Ergun, 1952]. The added mass force $F_{A}$ originates from the acceleration of discrete $\mathrm{CO}_{2}$ mass relative to the matrix fluid in the perturbed flow field [Wallis, 
1969]. Considering the spatial and transient nature of the rise velocity, linear momentum of $\mathrm{CO}_{2}$ rising in porous media is conserved following Equation 3.6:

$$
\sum F=\frac{D}{D t}\left(\rho_{C O_{2}} V u\right)=F_{A}+F_{b}+F_{d}+F_{s t}=\left(\rho_{C_{2}}+C_{M} \rho_{\text {matrix }}\right) V\left(\frac{\partial u}{\partial t}+u \frac{\partial u}{\partial y}\right)
$$

where $C_{M}$ is the added mass coefficient which depends on the geometry of the $\mathrm{CO}_{2}$ bubble, $\rho_{\mathrm{CO}_{2}}$ is the density of $\mathrm{CO}_{2}, \rho_{\text {matrix }}$ is the density of the matrix fluid, $V$ is the volume of $\mathrm{CO}_{2}$ bubble, and $u$ is the rise velocity of $\mathrm{CO}_{2}$. The added mass term is defined as:

$$
F_{A}=\frac{D}{D t}\left(C_{M} \rho_{\text {matrix }} V u\right)
$$

The buoyancy force is defined as:

$$
F_{b}=\left(\rho_{\text {matrix }}-\rho_{C O_{2}}\right) g V
$$

where $g$ is the acceleration of gravity. The surface tension force is:

$$
F_{s t}=2 \pi R_{P} \sigma \sin \theta
$$

where $\sigma$ is the surface tension at $\mathrm{CO}_{2}$-brine interface and can be described with Young-Laplace equation, $\theta$ is the equilibrium contact angle of $\mathrm{CO}_{2}$ contacting the wall of the pores. The drag force on the bubble of $\mathrm{CO}_{2}$ is defined as:

$$
F_{d}=A V\left[\frac{150 \mu_{\text {matrix }} u(1-\phi)^{2}}{d_{P}^{2} \phi^{3}}+\frac{1.75 \rho_{C O_{2}} u^{2}(1-\phi)}{d_{P} \phi^{3}}\right]
$$

where $A$ is the correction factor incorporating the effects of media-specific properties and contact pattern of $\mathrm{CO}_{2}$ with pore materials such as tortuosity, the shape factor and surface area [Cihan and Corapcioglu, 2008], $\phi$ is the effective porosity of the porous media, and $d_{P}$ is the mean diameter of the sedimentary particles. The drag term in this force balance is the most diffi- 
cult to quantify because the momentum transfer caused by drag force is impacted by heterogeneities in the pore geometry. Recognizing that drag force is associated with both viscous and kinetic energy losses, semi-empirical solutions to this term have been prepared such as those reported in the modified Ergun equation [Ergun, 1952]. The viscous energy losses are accounted by the Kozeny equation for laminar flow and the kinetic energy losses are described by Burke-Plummer equation for turbulent flow [Corapcioglu et al., 2004].

This theoretical methodology has been validated with experimental data reported in the literature including cases that are relevant to GCS case [Roosevelt and Corapcioglu, 1998]. After making one additional substitution for the volume of $\mathrm{CO}_{2}$ (i.e., $V=\frac{4}{3} \pi R_{\mathrm{CO}_{2}}{ }^{3}$ where $R_{\mathrm{CO}_{2}}$ is the equivalent radius of a sphere with a volume equal to that of a specified bubble) the conservation of momentum equation can be written as:

$$
\begin{aligned}
& \frac{4}{3} \pi R_{C O_{2}}{ }^{3}\left(\rho_{\text {matrix }}-\rho_{C O_{2}}\right) g-\frac{4}{3} \pi R_{C O_{2}}{ }^{3} A\left[\frac{150 \mu_{\text {matri } u} u(1-\phi)^{2}}{d_{P}{ }^{2} \phi^{3}}+\frac{1.75 \rho_{C O_{2}} u^{2}(1-\phi)}{d_{P} \phi^{3}}\right]-2 \pi R_{P} \sigma \sin \theta \\
& =\frac{4}{3} \pi R_{C O_{2}}{ }^{3}\left(\rho_{C O_{2}}+C_{M} \rho_{\text {matrix }}\right)\left(\frac{\partial u}{\partial t}+u \frac{\partial u}{\partial y}\right)
\end{aligned}
$$

The solution to this equation under steady state conditions (i.e., $\frac{D u}{D t}=0, C_{M}=0$ ) is the terminal velocity of $\mathrm{CO}_{2}$ bubble rise under channel or bubbly flow conditions and it can be written as follows [Corapcioglu et al., 2004]: 


$$
u=\frac{\mu_{\text {matrix }}(1-\phi)}{\rho_{\mathrm{CO}_{2}} d_{P}}\left[-42.857+\sqrt{1836.74-\frac{0.57}{A}\left(\frac{\rho_{\mathrm{CO}_{2}} d_{P}{ }^{3} \phi^{3}}{\mu_{\text {matrix }}{ }^{2}(1-\phi)^{3}}\right)\left(\frac{1.5 R_{P} \sigma \sin \theta}{R_{C O_{2}}{ }^{3}}-\left(\rho_{\text {matrix }}-\rho_{\mathrm{CO}_{2}}\right) g\right)}\right]
$$

Eq. 3.12

To illustrate how the rise velocity of leaked $\mathrm{CO}_{2}$ would behave in response to the viscosity measurements made here, Equation 3.12 was solved for the two leakage pathways presented in Figure 2.2. The characteristics for two representative geologic formations are also selected to understand the relative importance of geothermal gradients (Table 3.2) as compared to porosity, pore throat, particle size and $\mathrm{CO}_{2}$ bubble size (Table 3.3).

Table 3.2. Thermophysical properties used in Equation 3.12

\begin{tabular}{|c|c|c|c|c|}
\hline Parameters & Units & \multicolumn{2}{|c|}{ Values } & References \\
\hline Gravitational acceleration, $g$ & $\mathrm{~m} / \mathrm{S}^{2}$ & \multicolumn{2}{|c|}{9.81} & \\
\hline Density of brine, $\rho_{\text {matrix }}$ & $\mathrm{kg} / \mathrm{m}^{3}$ & \multicolumn{2}{|c|}{1020} & [Duan et al., 2006; Garcia, 2001] \\
\hline Correction factor, $A$ & & \multicolumn{2}{|c|}{26.8} & [Corapcioglu et al., 2004] \\
\hline & & \multirow{2}{*}{\multicolumn{2}{|c|}{0.04}} & [Chiquet et al., 2007b; Espinoza \\
\hline Interfacial tension, $\sigma$ & $\mathrm{N} \cdot \mathrm{m}$ & & & and Santamarina, 2010] \\
\hline \multirow[t]{3}{*}{ Contact angle, $\theta$} & Deg & \multicolumn{2}{|c|}{155.8} & Experimental Data \\
\hline & & \multicolumn{2}{|c|}{ Leakage Leakage } & \\
\hline & & \multicolumn{2}{|c|}{ Path a Path b } & \\
\hline Temperature, $T$ & ${ }^{\circ} \mathrm{C}$ & 40 & 20 & \\
\hline Pressure, $P$ & $\mathrm{MPa}$ & 5 & 5 & \\
\hline Density of $\mathrm{CO}_{2}, \rho_{\mathrm{CO}_{2}}$ & $\mathrm{~kg} / \mathrm{m}^{3}$ & 113.05 & 140.65 & [Span et al., 2000] \\
\hline
\end{tabular}


Table 3.3. Geological properties used in Equation 3.12

\begin{tabular}{|c|c|c|c|}
\hline Parameter & $\begin{array}{l}\text { Geological } \\
\text { condition } 1\end{array}$ & $\begin{array}{l}\text { Geological } \\
\text { condition } 2\end{array}$ & Reference \\
\hline Average porosity, $\bar{\phi}(\%)$ & 0.2386 & 0.3954 & $\begin{array}{c}\text { [Al-Raoush and Willson, 2005; Chen et } \\
\text { al., 2003; Padhy et al., 2007; } \\
\text { Widjajakusuma et al., 1999] }\end{array}$ \\
\hline Average particle size, $\overline{d_{p}}(\mathrm{~m})$ & 0.0025 & 0.004 & $\begin{array}{c}\text { [Corapcioglu et al., 2004; Glover and } \\
\text { Walker, 2011; Peters, 2009] }\end{array}$ \\
\hline Average pore throat, $\overline{R_{p}}(\mathrm{~m})$ & 0.001 & 0.0028 & $\begin{array}{c}\text { [Chen et al., 2003; Padhy et al., 2007; } \\
\text { Song, 2001] }\end{array}$ \\
\hline Average bubble radius, $\overline{R_{\mathrm{CO}_{2}}}(\mathrm{~m})$ & 0.0015 & 0.003 & Experimental data \\
\hline
\end{tabular}

The results, shown in Figure 3.13, demonstrate how rapidly rise velocity decreases with increases in matrix viscosity. For both geologic formations modeled here, an increase in viscosity from 0.65 to $5.42 \mathrm{mPa} \cdot \mathrm{s}$ can slow the terminal velocity up to $90 \%$. The rise velocity under geological condition 2, which represents coarse porous media, is about two orders of magnitude higher than that under geological condition 1 with all the other conditions remaining the same. Buoyancy has a much weaker effect on the rise velocity. Stronger buoyancy (leakage pathway a compared to leakage pathway b in Figure 2.2) increases the rise velocity only slightly. The extent of influence of buoyancy on the rise velocity varies with a more pronounced effect under the regime of fine porous media (geological condition 1) and with an insignificant effect under the regime of coarse porous media (geological condition 2). These results suggest that under realistic conditions, the viscosity of the matrix fluid affects the rise velocity independently of the geologi- 
cal, geothermal and hydrostatic conditions while the impact from buoyancy is more casespecific.
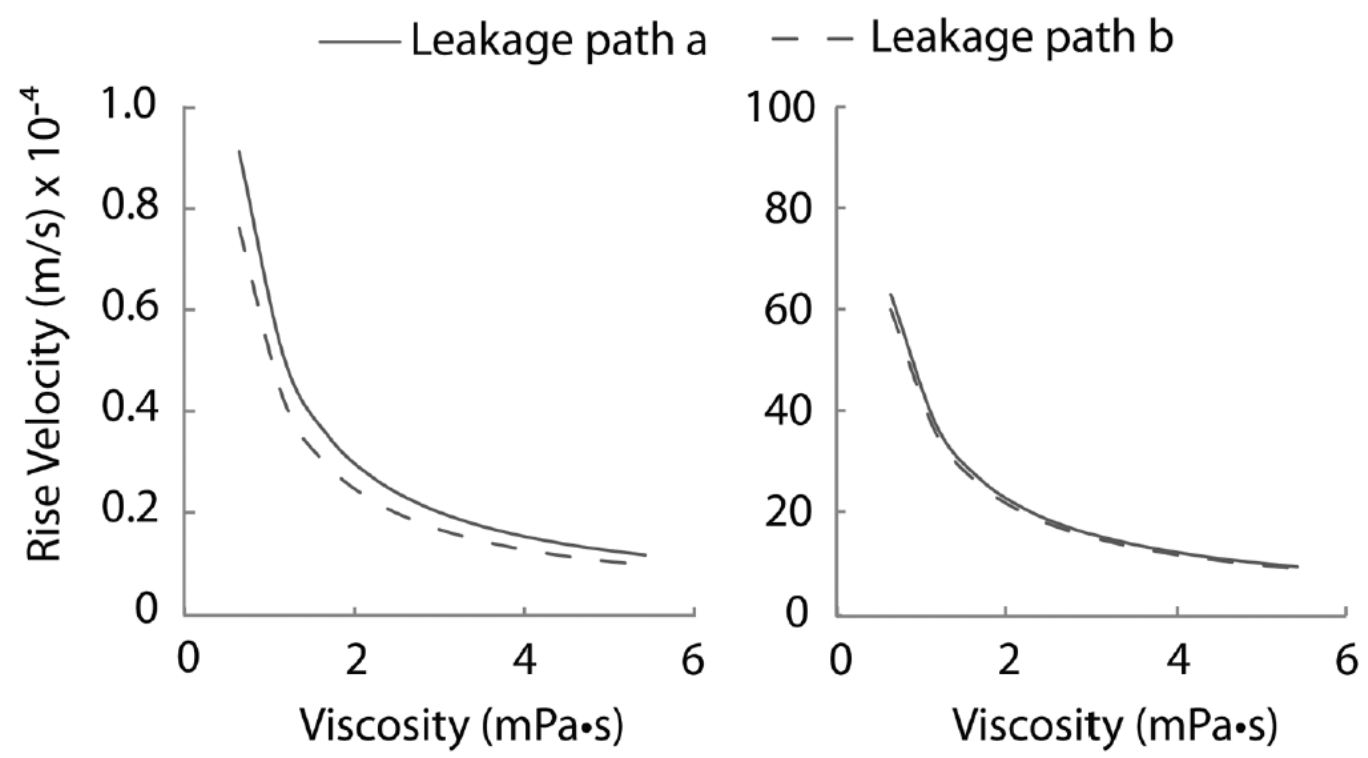

Figure 3.13. Rise velocity of leaked $\mathrm{CO}_{2}$ channel or bubbly flow through porous media obtained from Equation 3.12 using the viscosity data measured in this work following the two leakage pathways defined in Figure 2.2, under two specified geological conditions: (left) geological condition 1 for fine porous media; (right) geological condition 2 for coarse porous media. The characteristics of these geological conditions are listed in Table 3.3.

\subsection{Summary and Conclusions}

Taken together, the results of this work help paint a more complete picture of flow properties that are likely to influence the leakage processes that will impact the feasibility of geologic carbon sequestration. In particular, the results demonstrate that the ebullition and evolution of $\mathrm{CO}_{2(\mathrm{~g})}$ bubbles could act to impede vertical flow of $\mathrm{CO}_{2}$-brine mixtures through porous formations in as much as these bubbles will alter the effective viscosity of the mixture. The change 
in the viscosity will influence the force balance on a rising bubble of $\mathrm{CO}_{2}$ and this will have important implications for developing more accurate estimates of residence time that $\mathrm{CO}_{2}$ and brines will spend in contact with specific formations for investigating reaction kinetics and other associated processes. Further, this work cataloged the effect of shear, ionic strength, pressure and temperature on the flow properties of these $\mathrm{CO}_{2}$-brine mixtures. Existing models of complex multiphase flow were found to adequately predict how these mixtures will behave over a range of conditions. Finally, the experimental results demonstrate that the microstructure that results under VLE conditions in $\mathrm{CO}_{2}$-brine mixtures is strongly influenced by shear and that the bubbles are easily disrupted. This work combined experimental results with applicable modeling frameworks to provide a first look at how $\mathrm{CO}_{2}$ would travel over long distances. These results could aid in more thorough characterization of possible repositories. When assessing the viability of a potential GCS site, the thermal gradient in the subsurface must be considered together with specific formation characteristics (e.g., water saturation, permeability, porosity, stratification, etc.) and endogenous brine properties (e.g., ionic strength, $\mathrm{pH}$ ) to develop a better understanding of how $\mathrm{CO}_{2}$ might escape. 


\section{CHAPTER 4}

\section{Wettability Phenomena at the $\mathrm{CO}_{2}$-Brine-Mineral Interface}

Geologic carbon sequestration (GCS) in deep saline aquifers results in chemical and transport processes that are impacted by the wettability characteristics of formation solid phases that contain connate brines and injected $\mathrm{CO}_{2}$. Here, the contact angle $(\theta)$ at the $\mathrm{CO}_{2}$-brinemineral interface is studied for several representative solids including quartz, microcline, calcite, kaolinite, phlogopite, and illite under a range of conditions. All were found to be water wetting $\left(\theta<30^{\circ}\right)$ with subtle but important differences in contact angles observed between the surfaces. Temperature and pressure conditions affected the results but did not produce discernible trends common to all surfaces. Brine composition, in terms of $\mathrm{pH}$ and ionic strength, was a better predictor of interfacial behavior. For the non-clays, the wettability is impacted by the $\mathrm{pH}$ at the point of zero charge of the solid. For the clays, the response was more complex. Under nonequilibrium conditions, hysteretic effects were observed when $\mathrm{CO}_{2}$ was dissolving into the bulk fluid and this effect varied between minerals. Contact angle was found to decrease during the $\mathrm{CO}_{2}$ phase transition from supercritical or liquid phase to gas phase. These results are useful for developing a more complete understanding of leakage through caprocks and capillary trapping in GCS. 


\subsection{Materials and Methods}

\subsubsection{Contact Angle Measurements}

The three-phase contact angle between $\mathrm{CO}_{2}$-brine-mineral was measured in a custombuilt high-pressure cell (The design is provided in Appendix). The design was evolved from the existing methods in literature reports along with novelty and improvements [Yang et al., 2008]. Both pressure and temperature measurements were recorded using a National Instruments DAQ and LabView software. Pressure was collected using a Honeywell TJE 1240891 pressure transducer with in-line amplifier and controlled using a Teledyne ISCO 500HP syringe pump. Temperature was collected using an Omega K-type thermocouple and controlled using heating tape. The pressure cell contained a PTFE stage to hold mineral samples, a bottom injection port through which to add precise quantities of $\mathrm{CO}_{2}$ using the syringe pump and to add brine using a LabAlliance high pressure HPLC pump, and two sapphire windows at both ends to photograph the droplet. A stir bar was used to equilibrate the contents of the cell prior to a measurement. A Corr Instruments high temperature, high pressure glass bead $\mathrm{pH}$ probe was used to determine the $\mathrm{pH}$ in the vessel. The experimental setup is shown in Figure 4.1. The brine and $\mathrm{CO}_{2}$ delivery system and connection parts are presented in Appendix. A backpressure regulator was used as well during experiments. 


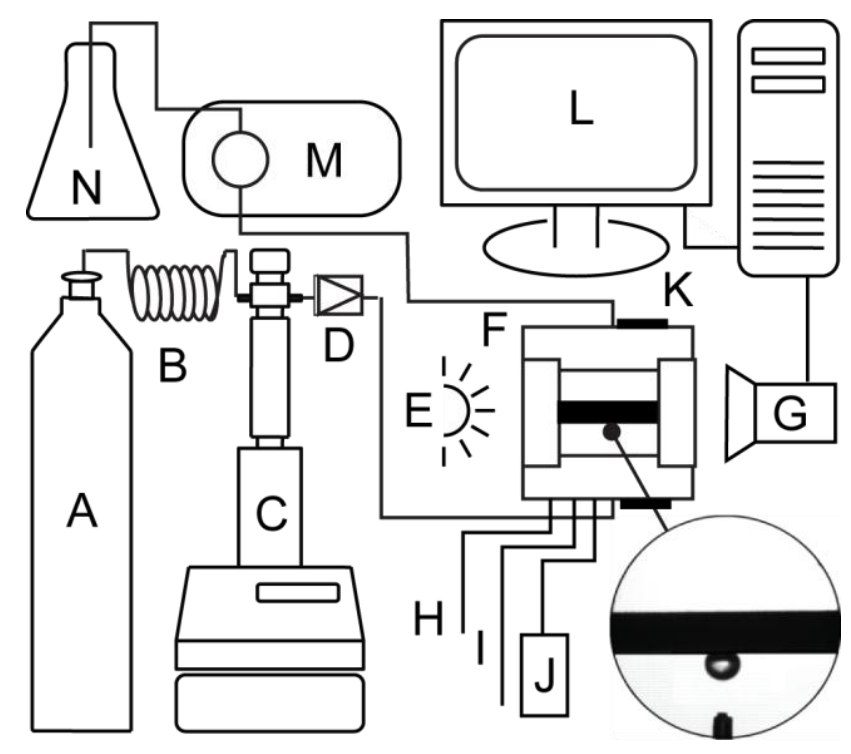

Figure 4.1. Experimental setup: $\mathrm{A}$ - tank of food grade $\mathrm{CO}_{2}, \mathrm{~B}$ - heat exchanger, $\mathrm{C}-$ syringe pump, D - one way valve, E - light source with diffuser, F - custom-built stainless steel 316 pressure vessel with sapphire windows, $\mathrm{G}$ - digital camera, $\mathrm{H}$ - thermocouple, I - high pressure $\mathrm{pH}$ probe, $\mathrm{J}$ - pressure transducer, $\mathrm{K}$ - heating tape, $\mathrm{L}$ - computer with DAQ, $\mathrm{M}$ - HPLC pump, $\mathrm{N}$ - brine.

Buoyant forces required the use of the captive bubble method to measure the $\mathrm{CO}_{2}$-brinemineral contact angle (Figure 4.2). $\mathrm{A} \mathrm{CO}_{2}$ bubble was introduced below a polished mineral sample surface. The mineral sample was fixed in place using a Teflon stage and the entire pressure vessel was positioned on a stability table to ensure that the bubble would be stationary over long (weeks) time scales. 


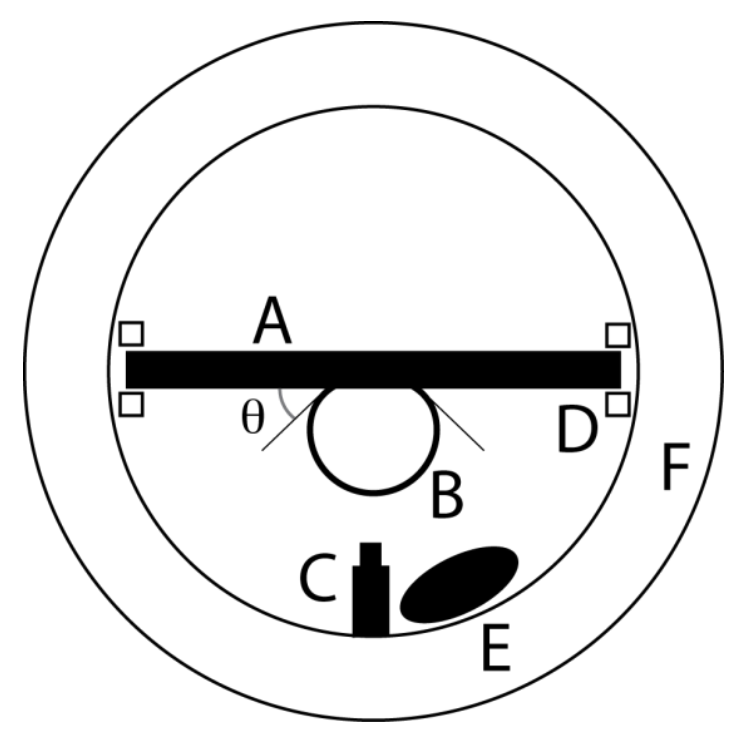

Figure 4.2. Captive bubble method for measuring contact angles of $\mathrm{CO}_{2}$ on mineral surfaces wetted with brine: $\mathrm{A}$ - mineral sample, $\mathrm{B}-\mathrm{CO}_{2}$ bubble, $\mathrm{C}$ - injection port, $\mathrm{D}$ - Teflon stage, $\mathrm{E}-$ stir bar coated with Teflon, $\mathrm{F}$ - pressure vessel wall.

Images of the captive bubble were collected using an AVT Guppy NIR CCD color camera with a 9.8-77 mm video zoom lens (Edmund Optics) and a light diffuser. The contact angle is measured directly from the photographs using image processing software (ImageJ) and axisymmetric drop shape analysis plugins (Dropsnake and LB-ADSA). The entire testbed was mounted on a stability table to permit for long-term (weeks) studies of the hysteresis of $\mathrm{CO}_{2}$ on different mineral samples. Both syringe pump and an invented "two-valve" control system were used to control $\mathrm{CO}_{2}$ droplets during the hysteresis (advancing and receding) tests. The invented "twovalve" control unit was demonstrated to be the best technique up to date to precisely control $\mathrm{CO}_{2}$ droplets by adding/withdrawing trivial amount of $\mathrm{CO}_{2}$ mass to/from the moving $\mathrm{CO}_{2}$ droplets. This was achieved by the unique advantage of the "two-valve" setting that very delicate pressure gradient $(<10 \mathrm{psi})$ could be repeatedly created by coordinating the two valves. The details are described in the Appendix. Contact angles on the left and right side of each bubble were record- 
ed and averaged. The minimum and maximum difference in values between the measured left and right contact angle is $0.02^{\circ}$ and $4.46^{\circ}$, respectively. Many precautions are needed to ensure appropriate handling of equipments and accurate data measurement. The details are provided in the Appendix.

\subsubsection{Minerals Studied Here}

Six minerals were considered in this work (Table 4.1), i.e., quartz, calcite, microcline, kaolinite, illite, and phlogopite, and were obtained from Ward's Natural Science. These six were selected because they were among the most representative minerals on the pore surface in the Viking sandstone [Peters, 2009]. The six minerals considered here also provide a range of surface characteristics and $\mathrm{pH}$ at the point of zero charge $\left(\mathrm{pH}_{\mathrm{PZC}}\right)$. All the minerals included here have hydroxide functional groups at the surface that are sensitive to the $\mathrm{pH}$ conditions of the adjacent wetting phase. The pHpzc for an aqueous solution in contact with a hydroxide mineral surface is defined when the surface concentration of $\left[=\mathrm{OH}_{2}{ }^{+}\right]=\left[=\mathrm{O}^{-}\right]$[Adamson, 1997]. All these minerals have this surface structure, as shown by the schematics of surface chemistry in Table 4.1, though differences exist between the atoms to which the oxygen atoms are bound. This range of surface properties allowed for hypothesis testing related to brine $\mathrm{pH}$ and buffering after equilibrating with $\mathrm{CO}_{2}$. The sample preparation method was modified from the literature [Yang et al., 2008]. Mineral samples were prepared by sectioning rocks, lapping them using diamond impregnated wheel, and then polishing them with silicon carbide sanding papers down to $25 \mu \mathrm{m}$ roughness to minimize the contact angle hysteresis due to heterogeneities in the surface. Extra

cares were taken to handle kaolinite because fractures could easily evolve under pressure and kaolinite is also known for its microporosity and sheet morphology. The samples were: 1) soaked 
in an acetone bath for 3 hours for nonclays and 0.5 hours for clays, 2) heated to $120^{\circ} \mathrm{C}$ for 2 hours, 3) sonicated in deionized water and 4) flushed with nitrogen to dry. At the beginning of an experiment, the high pressure vessel was heated and pressurized to the specified temperature and temperature and the sample was flushed by the tested solutions at a flow rate of $3 \mathrm{~mL} / \mathrm{min}$ for at least an hour to allow them to be well-adapted to the aqueous environment with the effective mixing from the stir bar. This step is also very important based on the fact that interfacial energy is sensitive to the dry or wet environment. This treatment effectively mimics the realistic sequestration reservoir conditions for the tested rocks. Surface roughness is yet to be accounted. External controls, separate samples of the same minerals, were tested to validate the method and these results are presented in the Appendix. It should be noted that in reality, many minerals are powder-shaped and cannot allow direct measurement by using the sectioned specimen method. Efforts have been made to clean and coat powdered minerals such as smectite and kaolin powders on high purity microscope slides using poly vinyl alcohol (PVA). The details can be found in the Appendix. 
Table 4.1. Representative minerals included in this analysis all have hydroxide surfaces that interact with connate brines based on the point of zero charge $\mathrm{pH}$ (pHpzc) of each mineral. pHpzc varies with temperature and ionic strength of the aqueous phase and the reported values are average under room temperature and pressure conditions.

\begin{tabular}{|c|c|c|c|c|c|}
\hline \multirow[t]{2}{*}{ Mineral } & \multicolumn{2}{|c|}{$\begin{array}{l}\text { Occurrence in } \\
\text { Sandstone* } \\
\text { [Peters, 2009] }\end{array}$} & \multirow[t]{2}{*}{ Molecular Formula } & \multirow[t]{2}{*}{ Surface Structure } & \multirow[t]{2}{*}{$\mathrm{pH}_{\mathrm{pzc}}$} \\
\hline & Bulk & Surface & & & \\
\hline Quartz & $73 \%$ & $17 \%$ & $\mathrm{SiO}_{2}$ & & $\begin{array}{c}2.9-3 \\
\text { [Brady, 1996; } \\
\text { Papirer, 2000; } \\
\text { Sverjensky, } \\
1994]\end{array}$ \\
\hline $\begin{array}{c}\text { Kaolinite } \\
\text { (1:1 layered) }\end{array}$ & $21 \%$ & $81 \%$ & $\mathrm{Al}_{2} \mathrm{Si}_{2} \mathrm{O}_{5}(\mathrm{OH})_{4}$ & & $\begin{array}{c}\text { 3.5-4.6 } \\
\text { [Sverjensky, } \\
\text { 1994; Schroth } \\
\text { and Sposito, } \\
\text { 1997] }\end{array}$ \\
\hline Calcite & $6 \%$ & $1.7 \%$ & $\mathrm{CaCO}_{3}$ & ó- & $\begin{array}{c}8-9.5 \\
\text { [Somasundaran } \\
\text { and Agar, } \\
\text { 1967; Stumn, } \\
\text { 1992] }\end{array}$ \\
\hline
\end{tabular}




\begin{tabular}{|c|c|c|c|}
\hline $\begin{array}{l}\text { Microcline } \\
\text { (K-feldspar) }\end{array}$ & $\mathrm{KAlSi}_{3} \mathrm{O}_{8}$ & 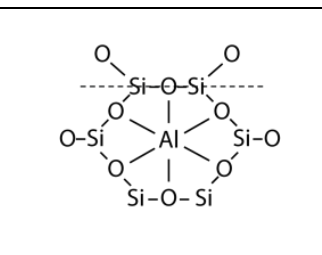 & $\begin{array}{c}2-2.4 \\
\text { [Sverjensky, } \\
1994]\end{array}$ \\
\hline $\begin{array}{c}\text { Illite } \\
(2: 1 \text { layered })\end{array}$ & $\begin{array}{c}\left(\mathrm{K}, \mathrm{H}_{3} \mathrm{O}\right)(\mathrm{Al}, \mathrm{Mg}, \mathrm{Fe}) \\
2 \\
(\mathrm{Si}, \mathrm{Al})_{4} \mathrm{O}_{10}\left[(\mathrm{OH})_{2}(\right. \\
\left.\left.\mathrm{H}_{2} \mathrm{O}\right)\right]\end{array}$ & 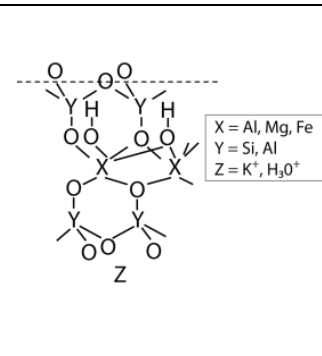 & $\begin{array}{c}2.4 \\
{[\text { Sverjensky, }} \\
\text { 1994] }\end{array}$ \\
\hline $\begin{array}{l}\text { Phlogopite } \\
\text { (2:1 layered) }\end{array}$ & $\begin{array}{c}\mathrm{KMg}_{3}\left(\mathrm{AlSi}_{3} \mathrm{O}_{10}\right)(\mathrm{F} \\
\mathrm{OH})_{2}\end{array}$ & 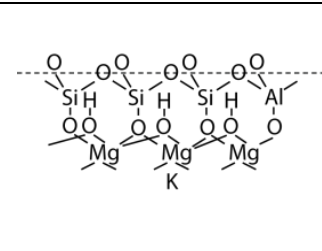 & $\begin{array}{c}8 \\
\text { [Sverjensky, } \\
\text { 1994] }\end{array}$ \\
\hline
\end{tabular}

*These estimates are for mineral classes and would include other related species. For example, kaolinite was grouped with chlorite, smectite, and albite.

\subsubsection{Experimental Conditions}

Pressure and temperature may have an important effect on contact angle measurements and so P-T conditions were selected to be representative for GCS but also to enable understanding of trends in P-T behavior, phase changes, and dissolution of $\mathrm{CO}_{2}$ in brine (Figure 4.3). The shallow conditions studied here (Case a: $30^{\circ} \mathrm{C}$ and $7 \mathrm{MPa}$ ) were selected to be near the triple point for $\mathrm{CO}_{2}$. Small changes in pressure and temperature near this point allow for the study of phase transitions needed to understand leakage processes. The deep conditions studied here (Case b: $50^{\circ} \mathrm{C}$ and $20 \mathrm{MPa}$ ) were chosen to mimic the environment near injection sites. These conditions are most useful for better understanding of residual and capillary trapping and other long-term storage processes. Note that pressure and temperature are not independent in the sub- 
surface since temperature rises in the subsurface as a result of geothermal gradients and hydrostatic forces increase pressure. Samples were considered in equilibrium after sitting in the vessel overnight ( 10 hours) with mechanical stirring. This time was determined experimentally during method development.

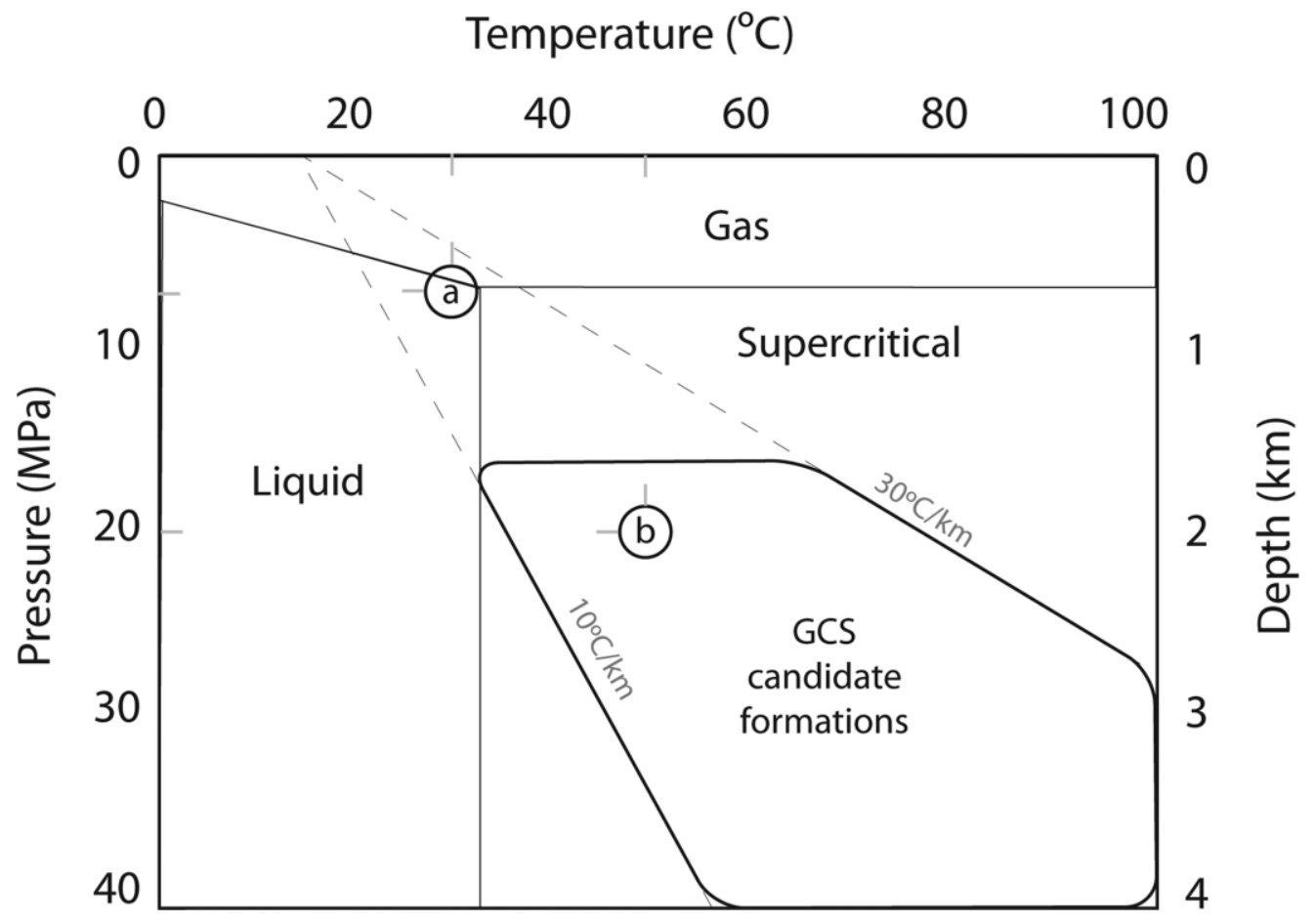

Figure 4.3. Pressure-Temperature conditions in the subsurface and the associated $\mathrm{CO}_{2}$ phase states investigated in this work.

\subsubsection{Brine Chemistry}

The chemistry of saline aquifers can vary considerably due to the characteristics of the host formation rocks [Morrow et al., 1998]. Here synthetic brines (Table 4.2) were used to mimic the composition of representative GCS formation pore waters [Mckaveney and Stivers, 1978]. Two brine chemistries were tested, one buffered and one without buffering. The non-buffered brine ( $\mathrm{pH}=7.8$ at RTP) contained $\mathrm{Na}^{+}, \mathrm{Cl}^{-}, \mathrm{Ca}^{+2}, \mathrm{SO}_{4}^{-2}$, and $\mathrm{Mg}^{+2}$ and had a $\mathrm{pH}$ of $\sim 3$ when con- 
tacted with high-pressure $\mathrm{CO}_{2}$. The buffered brine (pH=10 at RTP) contained $\mathrm{Na}^{+}, \mathrm{Cl}^{-}, \mathrm{Ca}^{+2}$, $\mathrm{SO}_{4}{ }^{-2}, \mathrm{Mg}^{+2}, \mathrm{CO}_{3}{ }^{-2}, \mathrm{HCO}_{3}{ }^{-}$, and $\mathrm{B}_{4} \mathrm{O}_{7}^{-2}$ and had a $\mathrm{pH}$ of $\sim 6$ when contacted with high-pressure $\mathrm{CO}_{2}$. Even though borate buffers have been shown to impact $\mathrm{CO}_{2}$ dissolution at higher pH values, at the high partial pressures of $\mathrm{CO}_{2}$ and the low $\mathrm{pH}$ ranges studied here, it was assumed that borate would not significantly impact aqueous $\mathrm{CO}_{2}$ concentrations [Livansky, 1982].

All brines were prepared in deionized water. The brines used here were based on recipes that were compiled from a large number of sources described in the paper and are provided below for reference. $\mathrm{pH}$ values are modeling estimates using the GWB-DUAN software and database under experimental conditions. These estimates should accurately reflect the exact pH values since the dissolution of mineral surface in the brine and the acid/base characteristics of that dissolution such as buffering have been accounted. 
Table 4.2. Brine recipes used here and the bulk brine properties including ionic strength and $\mathrm{pH}$.

\begin{tabular}{|c|c|c|c|c|}
\hline \multirow[b]{2}{*}{ Solution } & \multicolumn{2}{|c|}{ Recipe } & \multirow[b]{2}{*}{ Ionic strength $(\mathrm{mol} / \mathrm{L})$} & \multirow{2}{*}{$\begin{array}{l}\mathrm{pH} \text { under exper- } \\
\text { imental condi- } \\
\text { tions }\end{array}$} \\
\hline & Ionic species & $\begin{array}{c}\text { Concentration } \\
(\mathrm{mol} / \mathrm{L})\end{array}$ & & \\
\hline \multirow{5}{*}{ Brine } & $\mathrm{Na}^{+}$ & 1.022 & \multirow{5}{*}{$1.1 \sim 1.2$} & \multirow{5}{*}{$3.0 \sim 5.0$} \\
\hline & $\mathrm{Cl}^{-}$ & 1.0488 & & \\
\hline & $\mathrm{Ca}^{+2}$ & 0.0064 & & \\
\hline & $\mathrm{SO}_{4}^{-2}$ & 0.011 & & \\
\hline & $\mathrm{Mg}^{+2}$ & 0.018 & & \\
\hline \multirow{6}{*}{$\begin{array}{c}\text { Buffered } \\
\text { brine }\end{array}$} & $\mathrm{Na}^{+}$ & 0.8163 & \multirow{6}{*}{$7.3 \sim 7.5 \times 10^{-1}$} & \multirow{6}{*}{$5.8 \sim 5.9$} \\
\hline & $\mathrm{Cl}^{-}$ & 0.0625 & & \\
\hline & $\mathrm{SO}_{4}^{-2}$ & 0.011 & & \\
\hline & $\mathrm{CO}_{3}^{-2}$ & 0.294 & & \\
\hline & $\mathrm{HCO}_{3}^{-}$ & 0.043 & & \\
\hline & $\mathrm{B}_{4} \mathrm{O}_{7}^{-2}$ & 0.0504 & & \\
\hline DI water & N/A & N/A & $7.8 \times 10^{-4} \sim 9.9 \times 10^{-2}$ & $3.1 \sim 5.0$ \\
\hline
\end{tabular}




\subsection{Results and Discussion}

\subsubsection{Effect of Pressure, Temperature, Ionic Strength, and $\mathrm{pH}$ on Contact Angles at Equilibrium}

Equilibrium contact angle measurements were made first at the two pressure-temperature conditions in deionized water, brine, and buffered brine, respectively. The results, shown in Figure 4.4, indicate that these minerals are largely $\mathrm{CO}_{2}$ nonwetting (i.e., contact angles $<90^{\circ}$ ) with values generally ranging between $17^{\circ}$ and $28^{\circ}$. The magnitude of this difference (i.e., $\sim 38 \%$ ) is important and impacted by several factors including the mineral composition, the brine $\mathrm{pH}$ and ionic strength, and the P-T conditions. Published accounts of pressure swings contributing to a shift from nonwetting to intermediate wetting to wetting were not observed [Espinoza and Santamarina, 2010; Mills et al., 2011]. Though the higher temperature and pressure conditions in Figure $4.4 \mathrm{~b}$ clearly manifest in different ways for the minerals here, there was no consistent effect of pressure alone. 

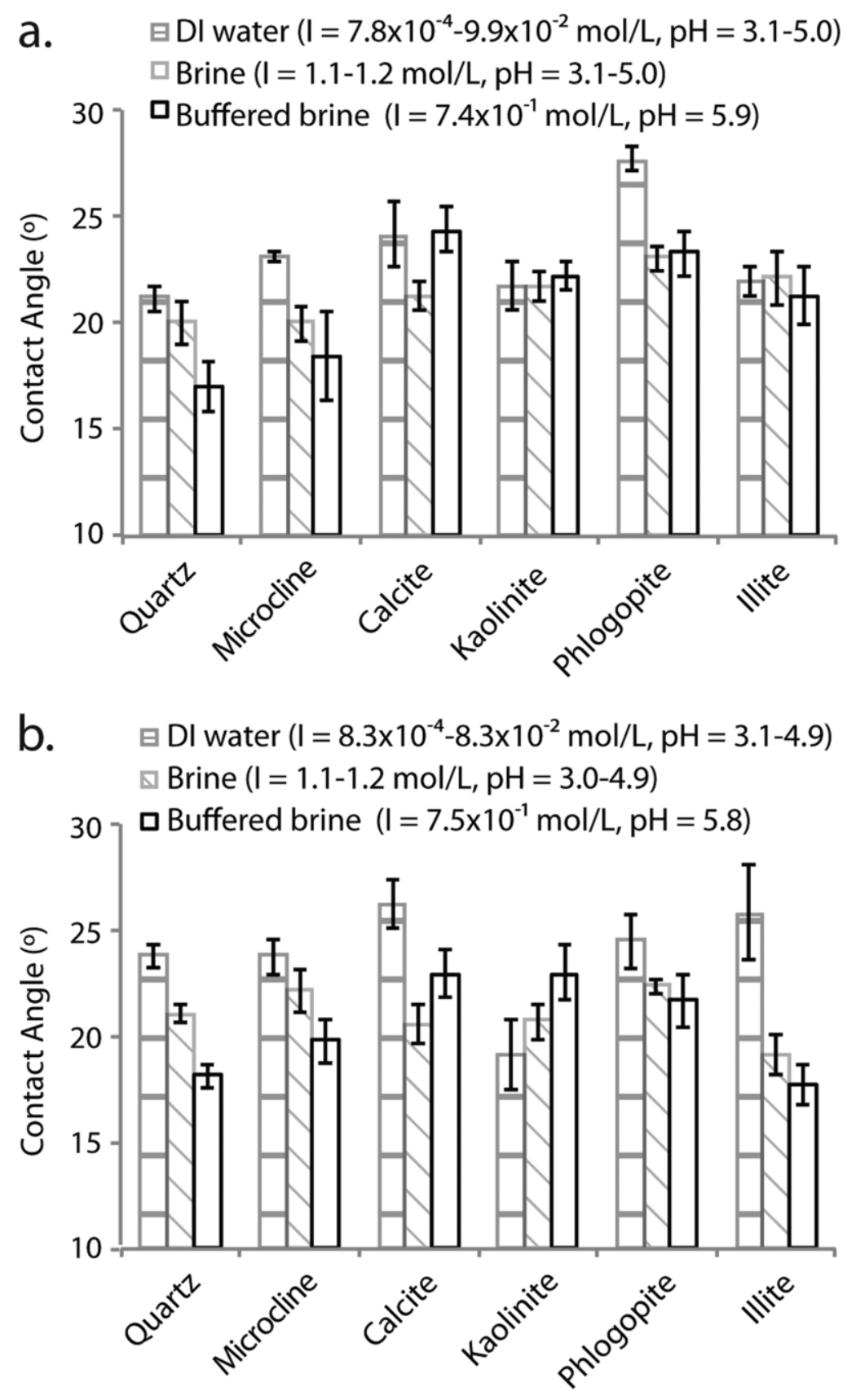

Figure 4.4. Equilibrium contact angle results for the six minerals considered here under low and high pressure-temperature conditions a and b shown in Figure 4.3, respectively, in three different solutions. Error bars represent $90 \%$ CI over at least eight replicate bubbles in two or more independent tests. 
The results of Figure 4.4 are useful for beginning to understand the effect of solution chemistry on contact angle on different surfaces. The two bars on the left for each mineral, i.e., horizontal hatches (left) and cross hatches (middle), are for deionized (DI) water and brine, respectively, at approximately the same $\mathrm{pH}$ but with different ionic strengths. Note that the values of $\mathrm{pH}$ and ionic strength vary slightly in Figures $4.4 \mathrm{a}$ relative to Figure $4.4 \mathrm{~b}$ because of the effect of higher $\mathrm{CO}_{2}$ partial pressure under these deep conditions. The two bars on the right for each mineral, i.e., cross hatches (middle) and white (right), are for brine and buffered brine, respectively, with comparable ionic strength but different $\mathrm{pH}$ conditions. The maximum difference in contact angles between surfaces under condition a and $\mathrm{b}$ are $8.6^{\circ}(32.7 \%)$ and $10.8^{\circ}(38.6 \%)$ respectively.

For the non-clays (i.e., quartz, microcline, and calcite), the $\mathrm{pH}$ of the brine was expected to influence the contact angle. It was hypothesized that as the solution approached the $\mathrm{pH}_{\mathrm{pzc}}$ for the mineral, the contact angle would increase. $\mathrm{CO}_{2}$ is a nonpolar molecule and it is more likely to interact with surfaces exhibiting a lower net surface charge. For all three minerals, the results are consistent and suggest that both $\mathrm{pH}$ and ionic strength have a role to play in driving contact angle. Both quartz and microcline have a low $\mathrm{pH}_{\mathrm{pzc}}$ and the contact angle decreases with increasing $\mathrm{pH}$. The results also support this hypothesis but in the opposite direction for calcite. Calcite has a high $\mathrm{pH}_{\mathrm{pzc}}$ so contact angle should increase as $\mathrm{pH}$ increases and the results confirm this behavior. Unlike the other non-clays, calcite does dissolve when contacted with low $\mathrm{pH}$ carbonated brine impacting both the ionic strength and pH of the solutions slightly [Zhang et al., 2010].

For the clays (i.e., kaolinite, phlogopite, and illite), the results are somewhat more complicated because of the nontrivial relationship between surface charge and acid/base chemistry in aluminosilicates. The $\mathrm{pH}_{\mathrm{pzc}}$ effect on wettability is impacted by secondary processes involving 
cation exchange between the clay and the brine [Duc et al., 2005]. Cation exchange would be expected to be more pronounced under low $\mathrm{pH}$ and low ionic strength conditions. The effect of these processes on $\mathrm{pH}_{\mathrm{pzc}}$, and surface charge more generally, are thought to impact 2:1 clays more significantly than 1:1 clays [Bleam, 1993]. The contact angle results support this mechanism. For the 2:1 clays, ionic strength impacts the contact angle under shallow conditions for phlogopite and deep conditions for illite. Kaolinite, which is known to have a $\mathrm{pH}_{\mathrm{pzc}}$ around 4, the contact angle was largely consistent over this $\mathrm{pH}$ range (3-5.9). A more delicate study on $\mathrm{pH}$ and ionic strength sweep tests of contact angle on clays are summarized in the Appendix.

The effect of precipitation/dissolution also likely impacts the contact angle at the clay interface. Precipitation/dissolution of phlogopite has been reported as a model mineral system in GCS [Giammar et al., 2005]. Shao et al. [2011] described the role that dissolved ions in solution can have on these reactions near the surface. These processes appear to impact contact angle, possibly by altering the double layer that drives the wettability of the mineral surface. This result was an example of the insight that can come from using the captive bubble method, wherein the clay is allowed to become wetted with brine with complete mixing provided by the stir bar before introducing the $\mathrm{CO}_{2}$ since the solution effects on the clay surface were more representative of in situ conditions.

Cation exchange is a very important indicator that links the chemistry we're seeing on the mineral surface with the aqueous chemistry that is occurring in solution. $\mathrm{K}^{+}$is one of the dominant cation species that exist in the crystal lattice of clay minerals and the cation exchange capacity (CEC) of $\mathrm{K}^{+}$for kaolinite, phlogopite, and illite clays was characterized using a $\mathrm{K}^{+}$selective electrode (Thermo Scientific, Inc.). The details are shown in the Appendix. These results shed 
light on producing a first principle understanding of how clays, which make up the majority of the pore surface in many sedimentary formations, will be impacted by solution chemistry.

\subsubsection{Salt Deposition and Surface Characterization}

Surface characterization on phlogopite was carried out to investigate potential surface reactions such as dissolution and precipitation. After experiments, the pressure vessel was very carefully and slowly depressurized and the sample was transmitted to conduct post-experiment characterization which was free of contamination.

SEM-EDS, AFM, Optical Microscopy analyses were conducted on sharply defined regions on the phlogopite sample surfaces where $\mathrm{CO}_{2}$ droplets resided during the experiments. Although it was challenging to preserve the trace of salt deposition after experiments, clear circular-shaped and irregular-shaped salt deposition sites which were the footprint of the triple line, surface defects such as small pit holes, cracks and local heights, and synergetic salt deposition around the surface defects were discovered (Figure 4.5 and 4.6). All these tests indicated that the surface of the phlogopite cleaved surfaces were homogenous and atomically smooth. Trivial amount of dusts and micro air bubbles were trapped between the sheets of phlogopite EDS analyses were conducted onto the phlogopite surface and the salt crystal. The results indicated that the salt crystal was $\mathrm{NaCl}$ and there was no chemicals captured as the product of phlogopite reaction. It has been acknowledged that surface roughness and defects and salt deposition could potentially cause wettability alteration. More details are shown in the Appendix. 


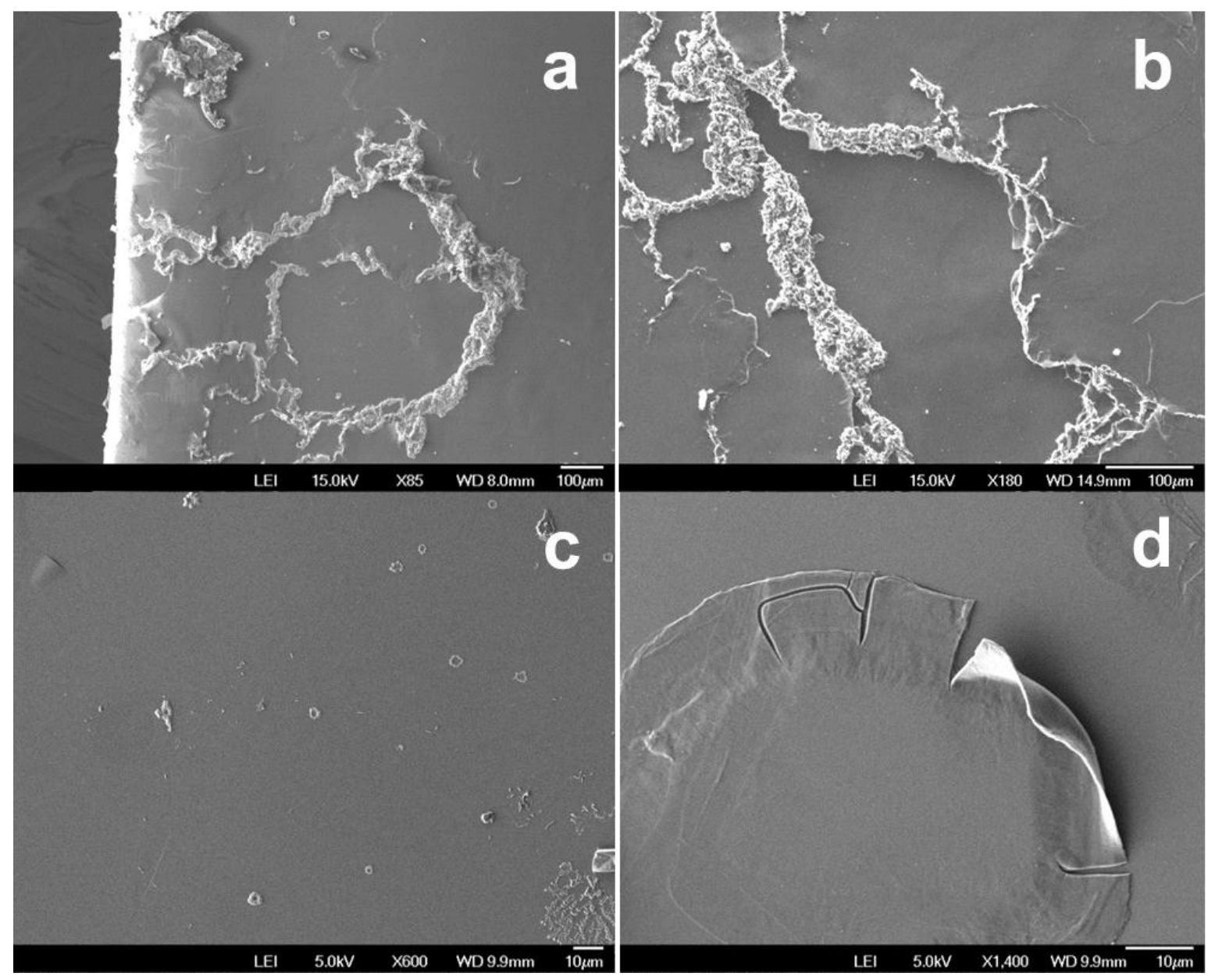

Figure 4.5. Surface characteristics of phlogopite after experiments with $\mathrm{CO}_{2}$ in $1.5 \mathrm{M} \mathrm{NaCl}$ solution under $20 \mathrm{MPa}$ and $50{ }^{\circ} \mathrm{C}$. (a) (b) and (c) circular shaped $\mathrm{NaCl}$ salt crystals were detected to form on the spots where $\mathrm{CO}_{2}$ had contacted and also preferentially form on surface defects and (d) the peel-off of phlogopite layer. Surface roughness, surface defects and salt deposition could potentially cause wettability alteration. 


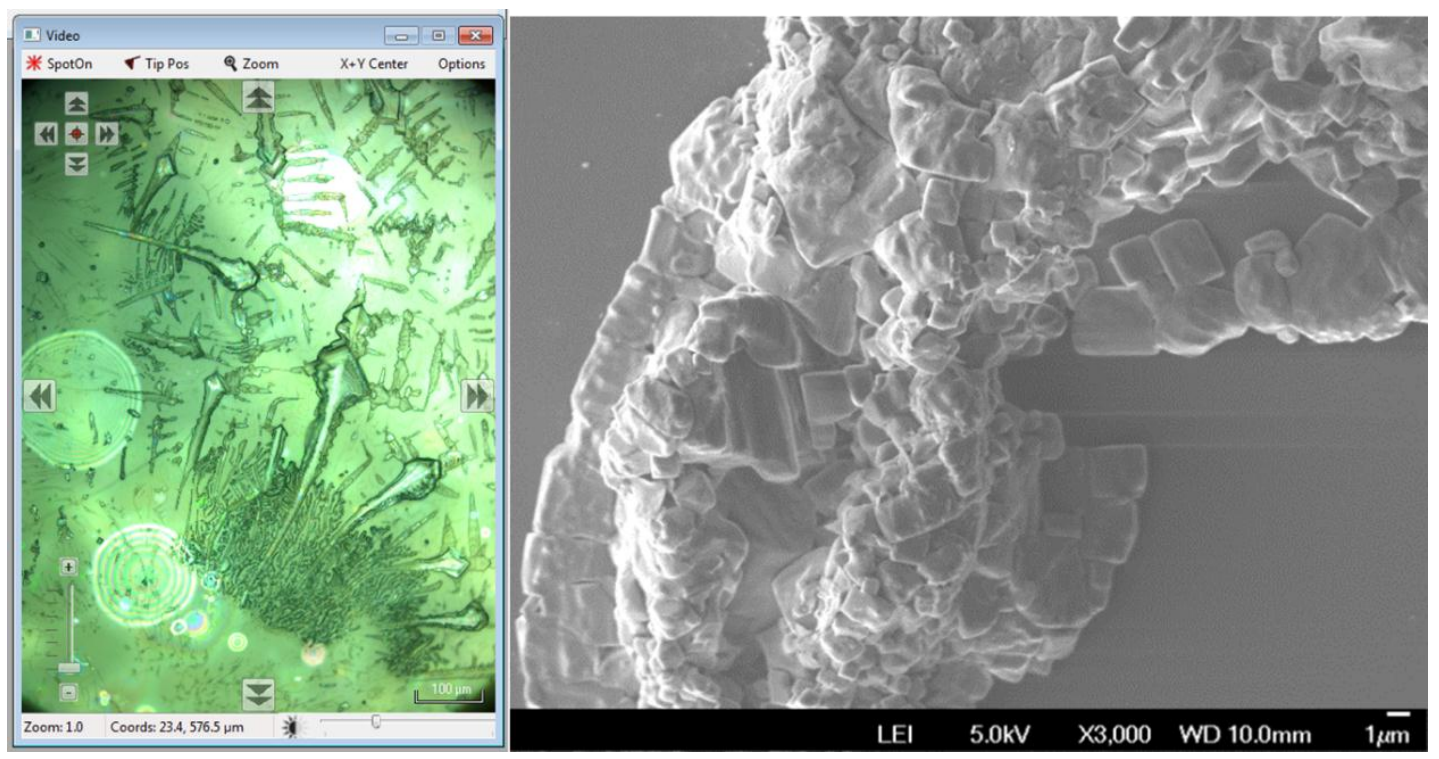

Figure 4.6. Both regular and irregular crystal structures were recorded in the experiments. (Left) AFM analysis revealed that snow-flaked shaped $\mathrm{NaCl}$ crystals had formed on phlogopite surface. (Right) SEM measurements showed highly regular $\mathrm{NaCl}$ crystals formed in part of the circular deposition.

\subsubsection{Wettability Map of Oil and $\mathrm{CO}_{2}$ on Quartz}

The relationship between solution chemistry and wettability has been explored for other relevant geochemical systems, most notably at the crude oil- brine-mineral interface. Figure 4.7a, a schematic of crude oil on a quartz surface, indicates that changes in $\mathrm{pH}$ and ionic strength result in a transition from oil wetting to oil-nonwetting. This transition has been described in terms of DLVO forces [Buckley et al., 1989]. At the $\mathrm{CO}_{2}$-brine-quartz interface, the changes in wettability over a similar range of aqueous conditions are much less pronounced (Figure $4.7 \mathrm{~b}$ ). No obvious transition from $\mathrm{CO}_{2}$-wetting to $\mathrm{CO}_{2}$-nonwetting is observed with the $\mathrm{CO}_{2}$ bubbles showing clear $\mathrm{CO}_{2}$-nonwetting behavior over the entire range of conditions. This result is consistent with past work that has shown $\mathrm{CO}_{2}$ to interact much more weakly with silica surfaces than other 
nonaqueous fluids [Tripp and Combes, 1998]. In practice, this suggests that significant transitions in wettability are unlikely for the material that constitutes the bulk of candidate formations and this could simplify efforts to model and understand transport and reaction mechanisms. Higher $\mathrm{pH}$ values were not measured here because the dissolved $\mathrm{CO}_{2}$ drives down the $\mathrm{pH}$ of the solution. The results in the next section indicate that a transition at very low $\mathrm{pH}$ is unlikely because of charge characteristics at the quartz surface.
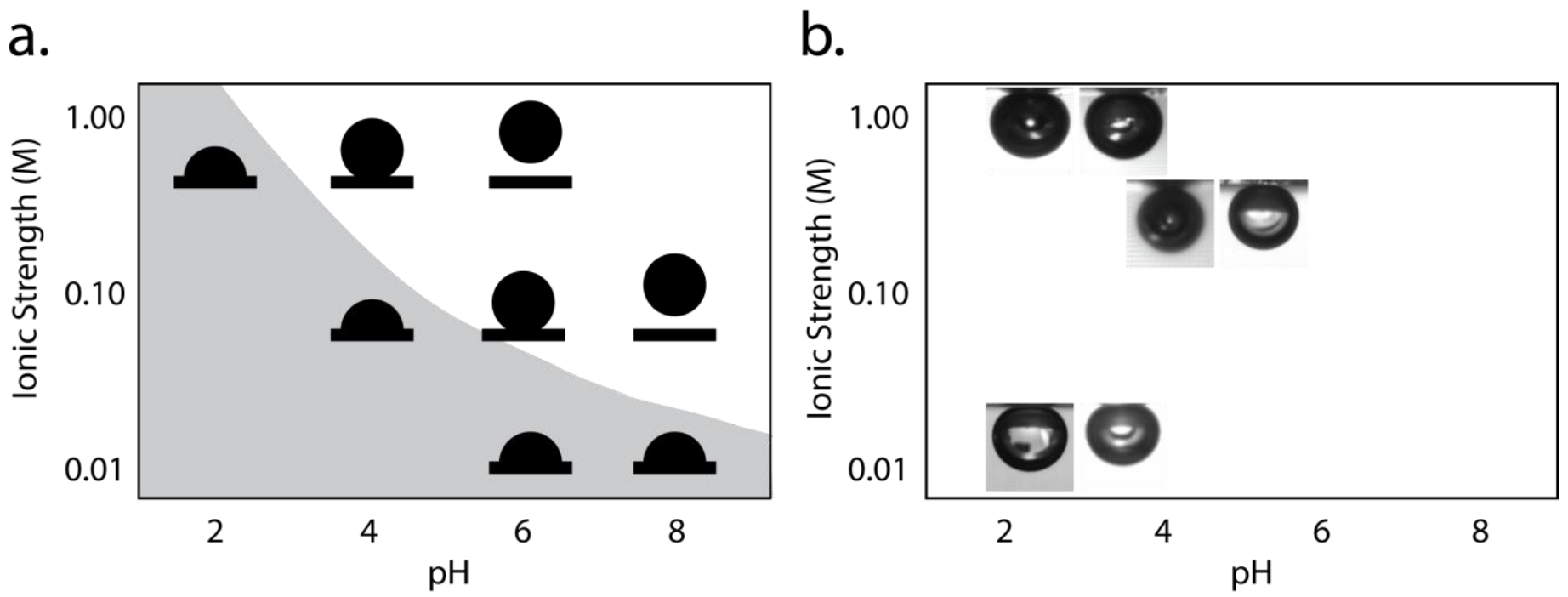

Figure 4.7. Wettability of (a) Alaska crude oil on quartz (adapted from [Buckley, 1996]) and (b) $\mathrm{CO}_{2}$ on quartz, both as a function of ionic strength and $\mathrm{pH}$. The grey region indicates the wettability transition for the crude oil on quartz. The data for (a) were collected at $27^{\circ} \mathrm{C}$ and the data for (b) were collected at $30^{\circ} \mathrm{C}$ (images on the right of each row) and $50^{\circ} \mathrm{C}$ (images on the left of each row). 


\subsection{4 pH Effects}

To better understand the effect of $\mathrm{pH}$ on contact angle, acid/base additions were performed for quartz, calcite, and microcline. These data were collected in non-buffered brine using $\mathrm{HCl}$ and $\mathrm{NaOH}$ to alter the $\mathrm{pH}$ from the equilibrium value of $\sim 3.1$ when the brine is contacted with high-pressure $\mathrm{CO}_{2}$. These $\mathrm{pH}$-modified brine solutions were added to the vessel using the HPLC pump and the contact angle was measured at each point of $\mathrm{pH}$. The results for quartz, shown in Figure 4.8a, suggest that the maximum contact angle occurs near the $\mathrm{pH}_{\mathrm{pzc}}$ of approximately 3. Figure $4.8 \mathrm{~b}$ shows the results for calcite $\left(\mathrm{pH}_{\mathrm{pzc}}=8-9.5\right)$ and here there is no inflection point, only an increase in contact angle as the $\mathrm{pH}$ of the system is raised. The overall $\mathrm{pH}$ range that could be covered in the calcite system is much lower because of the strong buffering capacity provided by the solid phase. In Figure $4.8 \mathrm{c}$, the results for microcline feldspar show a maximum contact angle near the $\mathrm{pH}_{\mathrm{pzc}}$ of 2-2.4. It should be noted that the published estimates for $\mathrm{pH}_{\mathrm{pzc}}$ are sensitive to pressure and temperature but the estimates used here are as reported in the literature, and this could represent a source of error. The larger trend, that $\mathrm{pH}_{\mathrm{pzc}}$ impacts contact angle, was consistent across these surfaces. This type of behavior has been reported for glass surfaces in aqueous solutions [Carre and Lacarriere, 2006] but not for the $\mathrm{CO}_{2}$-brine-mineral system. The $\mathrm{pH}$ and ionic strength sweep tests were also carried out on the surfaces of phlogopite, illite and kaolinite. The details are shown in Appendix. 

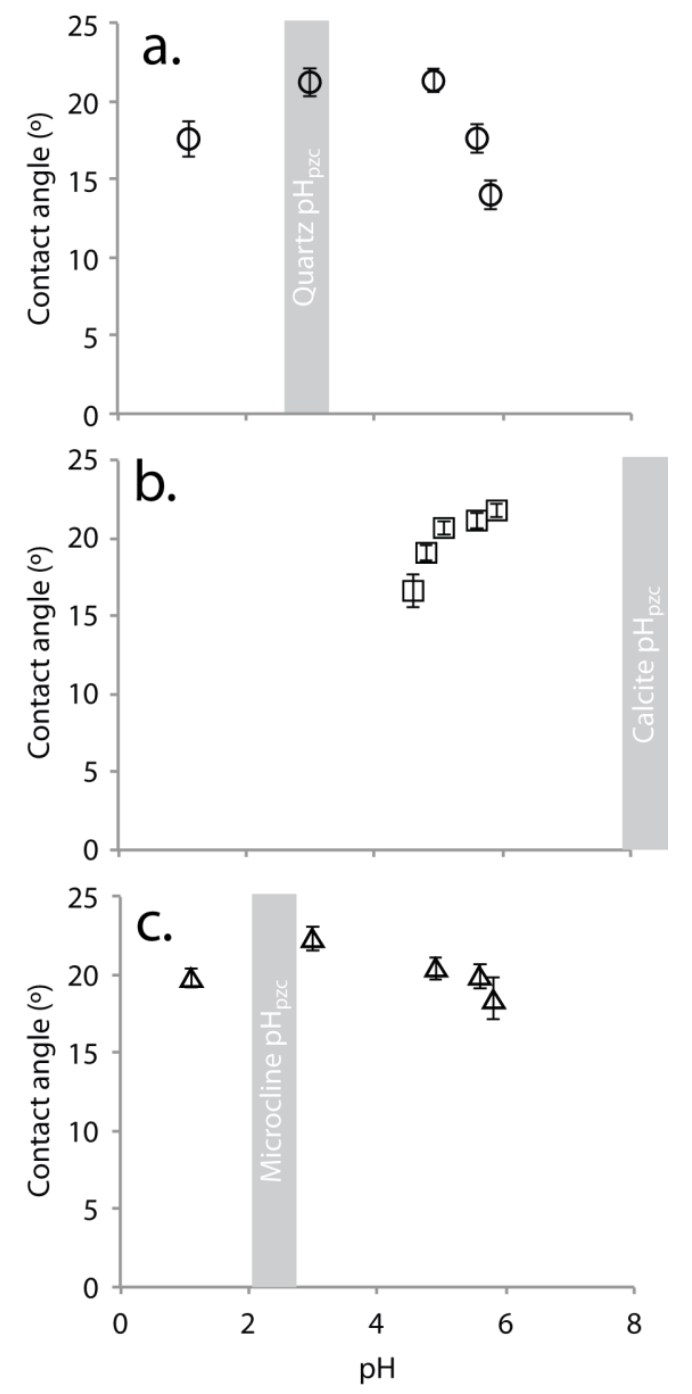

Figure 4.8. The addition of strong acids/bases suggested that contact angle is influenced by the $\mathrm{pH}_{\mathrm{pzc}}$ of a mineral surface. Here the results are for (a) quartz, (b) calcite and (c) microcline feldspar. All measurements were carried out for $\mathrm{I}=0.89-1.28 \mathrm{M}$ and pressure-temperature conditions b shown in Figure 4.3. 


\subsubsection{Hysteretic Effects}

Non-equilibrium conditions are expected to prevail near the leading edge and back end of a plume and so these conditions were measured here. Unlike conventional advancing/receding tests where a bubble is slowly applied to the mineral then drawn off the surface using the injection syringe, here hysteresis was measured by allowing a bubble of $\mathrm{CO}_{2}$ to dissolve into the brine. These conditions were expected to more closely approximate the processes that would exist near the boundaries of a plume of $\mathrm{CO}_{2}$ in the subsurface, i.e., wherein diffusion would dominate over advection in slow-moving aquifers. The diffusion of the $\mathrm{CO}_{2}$ into the brine was controlled over the course of a few minutes. It should be noted that contact angle hysteresis can refer to a variety of processes, including those driven by chemical and physical heterogeneities at the surface. The experiments here were designed to explore the GCS relevant hypothesis that mass transfer across the phase boundary would lead to a higher contact angle.

The results in Figure 4.9 show that the contact angle increases as the $\mathrm{CO}_{2}$ diffuses out of the bubble and that the extent and rate of increase is dependent on the type of mineral surface. Mineral surfaces that do not react with $\mathrm{CO}_{2}$, such as quartz have a minor change in contact angle (i.e., contact length/diameter ratio is constant), attributed primarily to the subtle change in size of the bubble. A more complete discussion of bubble size effects is provided in the Appendix. Only bubbles with very similar initial size were compared. For those minerals that do react with $\mathrm{CO}_{2}$, such as calcite, the effect of mass transfer on contact angle is more pronounced, and this is not attributed to bubble size changes. These experiments are functionally similar to receding contact angle measurements typically carried out in conventional contact angle hysteresis work and similar results have been reported for mica surfaces [Mills et al., 2011]. Even though the mass transfer occurring across the bubble surface could be indicative of contact line pinning, the corre- 
sponding decrease in contact length suggests this is not the case. The results were consistent at both conditions $\mathrm{a}$ and $\mathrm{b}$ in Figure 4.3.

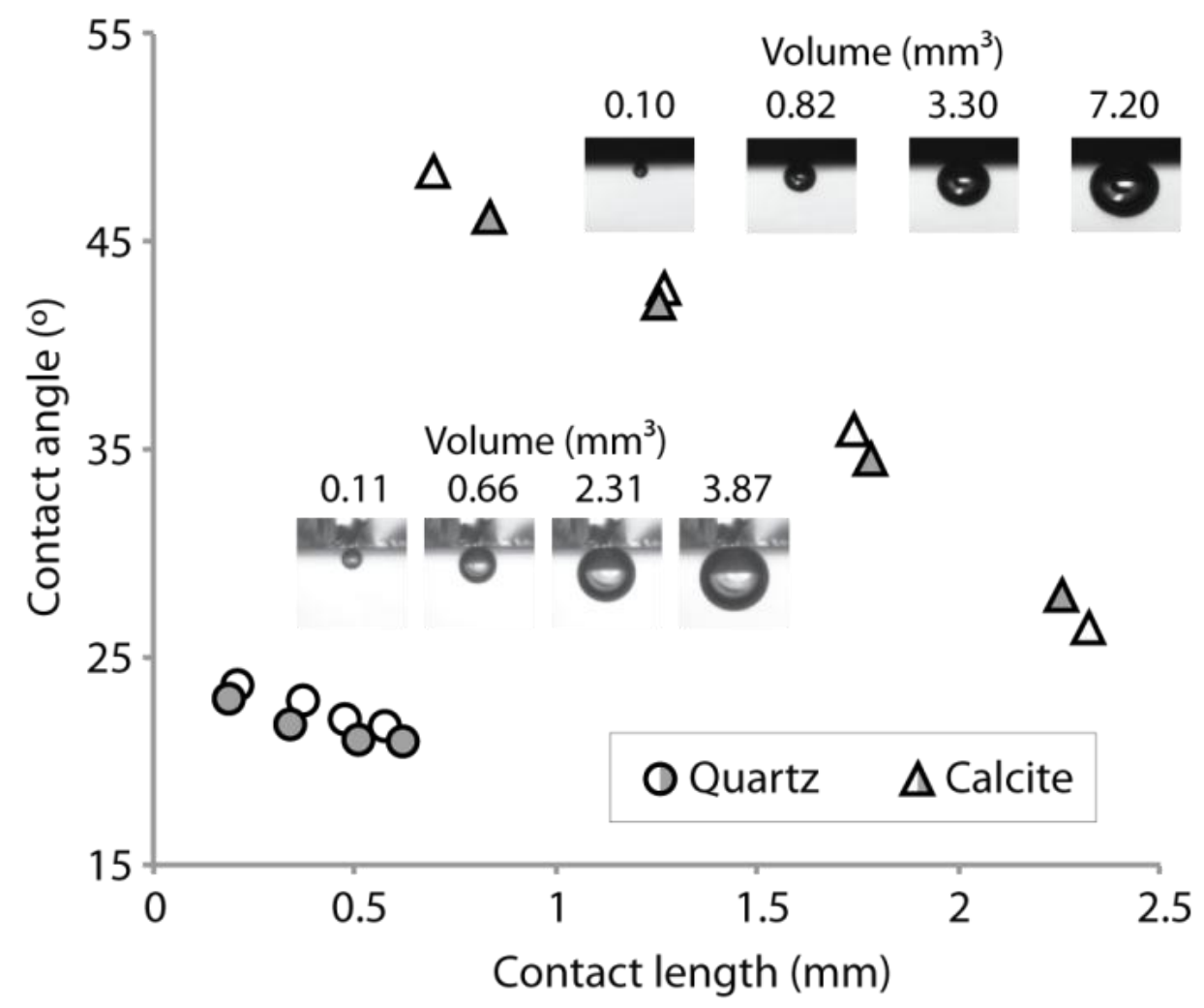

Figure 4.9. When $\mathrm{CO}_{2}$ is diffusing into the brine, the wettability is altered and the extent varies among mineral surfaces. Surfaces that react readily with $\mathrm{CO}_{2}$, such as calcite, have a much more pronounced hysteretic effect. These results were measured under pressure-temperature conditions b shown in Figure 4.3. Grey and white markers are for different replicates.

The contact line pinning was observed a number of times in this work (Figure 4.10). This processes has been described in the literature for other systems and has been explained in terms of mass transfer between the liquid phases with surface heterogeneities in natural sample composition (i.e., local defects) or surface roughness also playing a role [Moffat et al., 2009]. Though 
the process was difficult to reproduce, the behavior was observed fairly often (i.e., one out of every ten measurements) and as expected was most strongly influenced by the equilibrium state (e.g., how long the brine/ $\mathrm{CO}_{2}$ was allowed to equilibrate with the mineral surface) and on the mineral itself (e.g., the more reactive surfaces tended to demonstrate this behavior more often). More importantly, the contact line pinning was actually caused by the interfacial adhesion between $\mathrm{CO}_{2}$ and particular minerals within brine. The adhesion and contact line pinning was repeatedly observed in the adhesion research. Please refer to the next chapter for the details.

This pinning effect could influence the system at the leading and trailing edge of $\mathrm{CO}_{2}$ plumes before the brine and $\mathrm{CO}_{2}$ are not in equilibrium and where precipitation/dissolution reactions may be occurring. The extent to which this pinning will impact bulk flow properties in porous media remains to be established. On the one hand, the results here suggest that the effect will be small because it only occurs intermittently and under specific conditions. On the other hand, prior work has shown that when contact line pinning occurs on a large scale, capillary pressure can decrease significantly in porous media. This would greatly enhance the ability of $\mathrm{CO}_{2}$ to leak from host formations. From a practical standpoint, this effect is unlikely to dominate some of the more clear trends that were observed in this work e.g., $\mathrm{pH}$ effects on surface chemistry. But future work on the effects of brine/mineral chemistry and equilibrium conditions on pinning are warranted in light of these results. 
a.
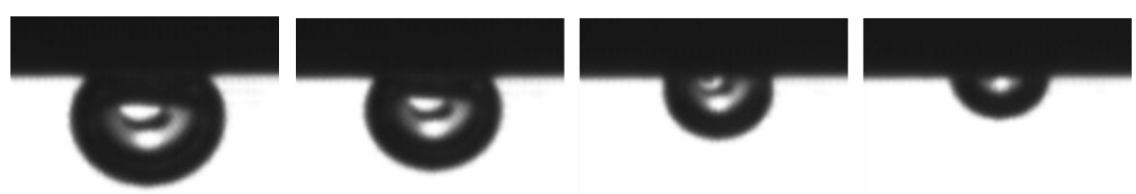

$\begin{array}{rr}\text { Contact line (mm) } & 1.63 \\ \text { Time (hours) } & 0.02 \\ \text { Contact angle }\left(^{\circ}\right) & 39.9\end{array}$

1.56
0.04
51.8

1.56

0.06

90.0

1.45

51.8

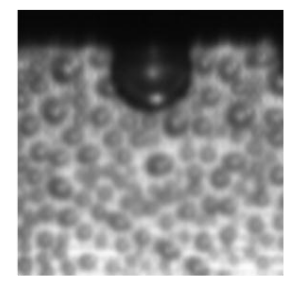

1.08

252 Time (hours) 1

Contact angle ( $\left.{ }^{\circ}\right) 22.2$
1.20

130

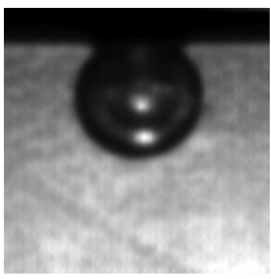

39.7
78.1
0.08

104.5

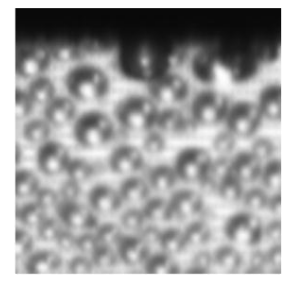

0.66

323

87.8

Figure 4.10. Contact line pinning phenomena were observed on different minerals over different time scales: a. calcite in deionized water under $7 \mathrm{MPa}, 30{ }^{\circ} \mathrm{C}$ (a few minutes), b. phlogopite in brine under $20 \mathrm{MPa}, 50{ }^{\circ} \mathrm{C}$ (weeks).

To further explore hysteresis conditions, several of the surfaces were left in contact with $\mathrm{CO}_{2}$ for up to three weeks. These experiments were carried out in equilibrium for calcite, quartz, and phlogopite. The hysteresis effect resulting from repeated exposure of mineral surfaces to acidified brine that was reported by Bikkina [2011] were not observed. No change was observed over this long time horizon for either calcite or quartz. These results were expected since hysteresis would be driven by precipitation/dissolution reactions and because these measurements were taken on pure surfaces, dissolution of the surface layer would reveal more of the same. The calcite samples lost significant mass over the course of the long experiments beneath the $\mathrm{CO}_{2}$ bubble. In practice, pore throats would have a different surface composition than the bulk media and if these surface minerals are subject to dissolution, the surface properties of the formation 
could change after extended exposure to $\mathrm{CO}_{2}$ (Table 4.1). Phlogopite did exhibit a drift in contact angle and the final contact angle was similar to that of kaolinite. Some hysteresis experiments did show interesting transitions from nonwetting to wetting characteristics, as previously noted in oil-quartz-brine systems by Buckley [1996], but these have been attributed to anomalies in the surface morphology and heterogeneity. A complete discussion of those results is provided in the Appendix.

\subsubsection{Phase Change inside $\mathrm{CO}_{2}$ Droplets and Its Effects}

The impact of fluid phase behavior on contact angles is known [Israelachvili, 2011] but the published results for the $\mathrm{CO}_{2}$-brine-mineral interface are limited [Espinoza and Santamarina, 2010; Bikkina, 2011; Chiquet et al., 2007a]. The interfacial characteristics of $\mathrm{CO}_{2(1)}$ and $\mathrm{CO}_{2(\mathrm{sc})}$ are similar when contacting brine while $\mathrm{CO}_{2(\mathrm{~g})}$ has much higher interfacial energy. It was hypothesized that the contact angle would change as $\mathrm{CO}_{2}$ transitions from supercritical or liquid to gas phase. The results in Figure 4.11 support this hypothesis as the contact angle decreases when $\mathrm{CO}_{2}$ transitions from liquid to gas phase. The results are obtained by adding cold $\left(4^{\circ} \mathrm{C}\right) \mathrm{CO}_{2}$, to a warm mineral surface at the GCS-relevant temperatures (pressure-temperature conditions a in Figure 4.3). Even though overall effect on contact angle is subtle, the result is consistent across a variety of mineral surfaces. This behavior may have important implications for modeling leakage processes. If $\mathrm{CO}_{2(\mathrm{~g})}$ is less wetting than $\mathrm{CO}_{2(1)}$, this could act as a resistance near the depth at which phase transition occurs. In practice the effect will need to be weighed against other factors that are likely to influence estimates of leakage (e.g., changes in density) that accompany phase transitions. 


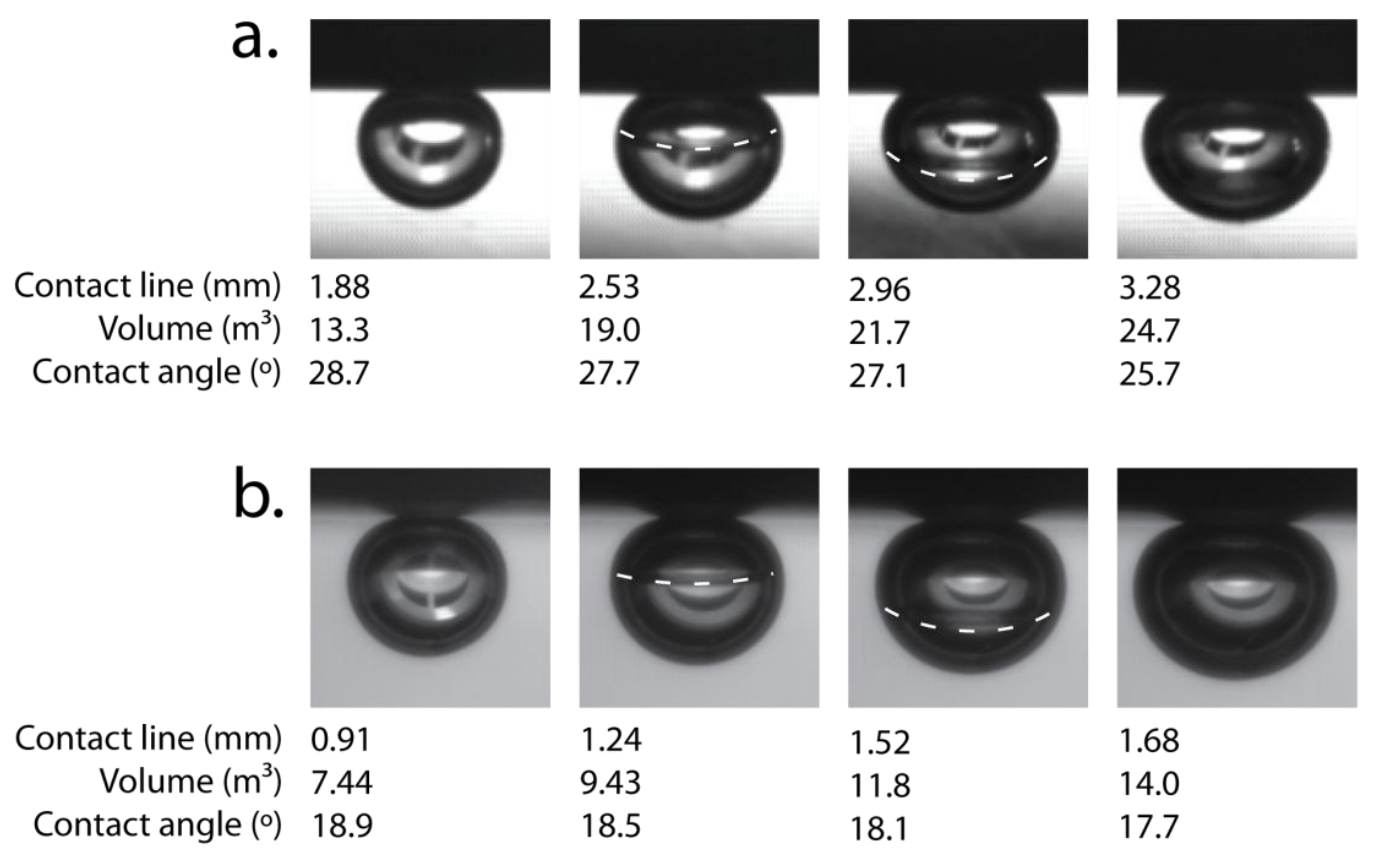

Figure 4.11. Contact angles decrease as $\mathrm{CO}_{2}$ transitions from the liquid or supercritical phase into the gas phase for (a) calcite and (b) microcline both in brine (pressure-temperature conditions a in Figure 4.3). The dotted lines highlight the movement of the phase boundary.

\subsection{Implications for GCS}

The wettability results reported here have particular relevance for at least two classes of problems that are important in GCS. The first is for assessing the sealing capacity of caprocks. The maximum storage pressure that caprocks can withstand during injection of $\mathrm{CO}_{2}$ has been the subject of intense study and is known to depend on the capillary pressure, which is proportional to the cosine of contact angle [Chiquet et al., 2007a]. Consequently, the effect of contact angle is non-linear and the effect of contact angle is more important for caprocks that have relatively small pore throats. The second class of problems in which these wettability data are important is in calculating the capillary/residual trapping of $\mathrm{CO}_{2}$ on dead-end pores far from the injection 
well. In these regions, where the pressure gradient from injection is small, wettability will significantly impact how much $\mathrm{CO}_{2}$ can be stored in a formation. This is important not only for estimating the capacity of target formations, but also for estimating how much $\mathrm{CO}_{2}$ adjacent aquifers can trap when/if leakage occurs.

To help contextualize the results of this work, the equations used to calculate these parameters are presented below and their sensitivity to contact angles is discussed. The parameters used in these calculations are presented in Table 4.3.

Table 4.3. Parameters used in implication calculation

\begin{tabular}{|c|c|c|c|}
\hline Parameters & Units & \multicolumn{2}{|c|}{ Values } \\
\hline gravitational acceleration, $g$ & $\mathrm{~m} / \mathrm{S}^{2}$ & \multicolumn{2}{|c|}{9.81} \\
\hline density of brine, $\rho_{\text {brine }}$ & $\mathrm{kg} / \mathrm{m}^{3}$ & \multicolumn{2}{|c|}{1020 [Garcia, 2001] } \\
\hline Porosity, $\phi$ & $\%$ & \multicolumn{2}{|c|}{30 [Padhy et al., 2007] } \\
\hline Residual brine saturation, $S_{\text {brine }}$ & $\%$ & \multicolumn{2}{|c|}{10 [Chalbaud et al., 2007b] } \\
\hline Surface tension, $\sigma$ & $\mathrm{N} \cdot \mathrm{m}$ & \multicolumn{2}{|c|}{0.028 [Espinoza and Santamarina., 2010] } \\
\hline & & Low & High \\
\hline Temperature, $T$ & ${ }^{\circ} \mathrm{C}$ & 30 & 50 \\
\hline Pressure, $P$ & $\mathrm{MPa}$ & 7 & 20 \\
\hline Depth, $d$ & $\mathrm{~m}$ & 700 & 2000 \\
\hline Pore throat, $R_{\text {throat }}^{\max }$ & $\mathrm{m}$ & $\begin{array}{c}5 \times 10^{-7} \\
{[\text { Padhy et al., 2007] }}\end{array}$ & $\begin{array}{c}3 \times 10^{-6} \\
{[\text { Padhy et al., 2007] }}\end{array}$ \\
\hline density of $\mathrm{CO}_{2}, \rho_{\mathrm{CO}_{2}}$ & $\mathrm{~kg} / \mathrm{m}^{3}$ & $\begin{array}{c}266.56 \\
\text { [Span et al., 2000] }\end{array}$ & $\begin{array}{c}784.29 \\
\text { [Span et al., 2000] }\end{array}$ \\
\hline
\end{tabular}




\subsubsection{Heterogeneity of Pore Surfaces}

The literature has shown and the data here support that most of the mineral surfaces found in GCS formations are largely water wetting and this will have implications for the amount of $\mathrm{CO}_{2}$ that can be stored in a formation but also for the ease with which $\mathrm{CO}_{2}$ can leak from these formations. To provide more resolution, the library of contact angles for clay minerals reported here will be especially useful. For a smooth but chemically heterogeneous surface, the Cassie equation (Eq. 4.1, adopted from [Chalbaud et al., 2007b]) is used for calculating the apparent contact angle.

$$
\cos \theta_{\text {apparent }}=\sum_{i} \chi_{i} \cos \theta_{i} \quad \text { Eq. } 4.1
$$

where $\cos \theta_{\text {apparent }}$ is the cosine of the apparent contact angle for the heterogeneous surface, $\mathcal{X}_{i}$ is the area fraction of homogeneous mineral species, and $\cos \theta_{i}$ is the cosine of the contact angle for the homogeneous mineral species $i$. As discussed in the text, the contact angle for the large majority of the diagenetic materials that are found on pore surfaces have not been reported and this work was performed to help fill that gap. For condition a and b, the cosine of the apparent contact angles for DI water, brine and buffered brine ranges from 0.9224 to 0.9354 , corresponding to the apparent contact angles $22.0^{\circ}, 22.0^{\circ}$ and $22.3^{\circ}$, and $20.7^{\circ}, 22.7^{\circ}$ and $21.3^{\circ}$ respectively, using the distribution of solids listed in Table 4.1. This suggests only a modest effect from salinity and depth, but the values are somewhat different from the average value of $30^{\circ}$ that is often used in many modeling analyses. 


\subsubsection{Caprock Integrity}

The pressure inside GCS target formations will be increased considerably near injection wells and the capacity of the caprock to support this pressure increase is a function of the capillary pressure, which can be calculated using the Young-Laplace equation (Eq. 4.2, adapted from [Chalbaud et al., 2007a]:

$$
P_{C}=P_{\mathrm{CO}_{2}}-P_{\text {brine }}=\frac{2 \sigma_{\text {brine }-\mathrm{CO}_{2}} \cos \theta}{R_{\text {throat }}^{\max }} \quad \text { Eq. } 4.2
$$

where, $P_{C}$ is the capillary pressure, $\sigma_{\text {brine-CO}}$ is the interfacial tension between endogenous brine and $\mathrm{CO}_{2}, \theta$ is the contact angle, and $\boldsymbol{R}_{\text {throat }}^{\max }$ is the largest interconnected pore throat or microfractures in the porous media. The capillary pressure for a caprock is proportional to the wettability of the material on the pore surface. But this proportionality is nonlinear because of the cosine and for caprock where the pore throat size is likely to be small, the influence of contact angle will be large. Two representative pore throat sizes, i.e., $3 \mu \mathrm{m}$ and $0.5 \mu \mathrm{m}$ were used and the calculated capillary pressure lies between 0.02 and $0.11 \mathrm{MPa}$ using the conditions low and high in Table 4.3 .

Another way to think about caprock integrity is to calculate the $\mathrm{CO}_{2}$ column height that can be stored in a reservoir. This can be calculated using Eq. 4.3, adapted from [Chiquet and Broseta, 2005]:

$$
h=\frac{2 \sigma_{\text {brine }-\mathrm{CO}_{2}} \cos \theta}{\left(\rho_{\text {brine }}-\rho_{\mathrm{CO}_{2}}\right) g R_{\text {throat }}^{\max }}
$$


where $h$ is the $\mathrm{CO}_{2}$ column height, $\rho_{\text {brine }}$ and $\rho_{\mathrm{CO}_{2}}$ are the densities of brine and $\mathrm{CO}_{2}$ respectively, and $g$ is the gravitational acceleration.

\subsubsection{Capillary/Residual Trapping}

A second broad class of problems that is highly relevant for GCS and for which contact angle is important is capillary/residual trapping. The capillary pressure can be scaled up and expressed as the reservoir storage capacity. This storage capacity, particularly in reservoirs that are the target for the injection, can be calculated as a mass of $\mathrm{CO}_{2}$ per surface area of the reservoir using Eq. 4.4, adapted from [Chiquet and Broseta, 2005]:

$$
M=\rho_{C O_{2}} h \phi\left(1-S_{\text {brine }}\right)=\frac{2 \sigma_{\text {brine-CO }} \cos \theta \rho_{\mathrm{CO}_{2}} \phi\left(1-S_{\text {brine }}\right)}{\left(\rho_{\text {brine }}-\rho_{\mathrm{CO}_{2}}\right) g R_{\text {throat }}^{\max }}
$$

where $\phi$ is the effective porosity and $S_{\text {brine }}$ is the saturation of the residual brine.

Using the data in Table 4.3, capillary pressure, $\mathrm{CO}_{2}$ column height, and residual trapping, were calculated for conditions a and b under large and small capillary size scenarios. Result shows, not surprisingly, that the contact angle results matter the most for formations with the lowest pore sizes. Sensitivity analysis suggests that the $38 \%$ differences in contact angle reported here could manifest in 5-10\% differences in capillary trapping or pressure. This estimate could help set the expectations in terms of using these data to better predict macro-scale behavior. These data will be useful for developing site-specific models to predict preferential flow pathways in time and space and help bridge the gap between existing modeling frameworks and the large-scale demonstrations of GCS. 


\section{Chapter 5}

\section{Interfacial Adhesion}

Capillary trapping, and the interfacial adhesive forces that drive it, could play an important role in determining the viability of geologic carbon sequestration (GCS). Here, adhesion at the $\mathrm{CO}_{2}$-mineral interface was been observed for the first time on trioctahedral phlogopite mica and amorphous silica surfaces. Using contact angle measurements of discrete captive bubbles on homogenous mineral surfaces, the effects of advancing and receding dynamics, as well as solution chemistry were recorded. For phlogopite and silica surfaces, a reproducible and significant effect was observed for bubbles that adhered to the surface. For phlogopite in contact with connate brines over $50 \%$ of bubbles adhered to the surface while over $70 \%$ of the bubbles on silica adhered. These results represent the aggregate understanding developed by observing thousands of bubbles on dozens of replicates on various representative mineral surfaces. $\mathrm{CO}_{2}$ bubbles adhered to the surface typically showed distorted ternary phase lines suggesting that some degree of contact line pinning was taking place. In advancing/receding contact angle measurements, adhesion could increase the contact angle by a factor of 3 and drive a transition from non-wetting to wetting. These bubbles were not easily removed from the surface unlike bubbles on surfaces 
that did not exhibit adhesion. Measurements on illite, kaolinite, quartz and calcite showed little to no adhesion. The incidence of adhesion increased with ionic strength in the solution. The carbonation of brines also enhanced adhesion. Adhesion was not observed in brines where $0.1 \mathrm{M}$ of strong acid or base had been added to the synthetic brine used here. Several factors could be driving this behavior including surface morphology, surface potential and affinity of the mineral surfaces for $\mathrm{CO}_{2}$. Even though the underlying mechanism driving this phenomenon is still being explored, the results could have important implications for understanding a variety of processes related to capillary trapping under GCS conditions. These results could be particular important for characterizing leakage processes wherein the ability for residual trapping of fugitive emissions will be of great interest for understanding how porous formations overlaying caprocks might surface to suppress gas leakage to the atmosphere.

\subsection{Background}

The forces that predominate at the $\mathrm{CO}_{2}$-brine-mineral interface are relevant to a number of processes including geologic carbon sequestration (GCS), enhanced oil recovery, and hydraulic fracturing. These forces have not been widely studied because mineral surfaces tend to be predominantly hydrophilic under most geologic conditions. Under multiphase flow conditions involving $\mathrm{CO}_{2}$, oil, methane, or other nonpolar molecules, the surface would be expected to largely maintain its water wetting characteristics. For the case of GCS, most work has suggested that differences in the solution chemistry (e.g., $\mathrm{pH}$, ionic strength) and the mineral composition at the pore surface (e.g., quartz, kaolinite, silica) could lead to minor differences in wettability 
[Mills et al., 2011; Wang, Edwards et al., 2013]. These changes could manifest modestly on macro-scale flow properties like capillary pressure and residual saturation.

A growing number of studies exploring the $\mathrm{CO}_{2}$-brine-mineral interfaces under $\mathrm{GCS}$ and EOR conditions have reported more dramatic shifts in wettability under certain conditions. On muscovite mica, Chiquet et al. reported significant wettability alteration along with adhesion and noticed the effect of pressure favoring these phenomena [2007a]. Broseta et al. explored the role of dense acid gases, e.g., $\mathrm{CO}_{2}$ and $\mathrm{H}_{2} \mathrm{~S}$ on altering wetting behaviors under GCS-relevant conditions [2012]. Using contact angle hysteresis (advancing-receding) experiments, wherein a captive $\mathrm{CO}_{2} / \mathrm{H}_{2} \mathrm{~S}$ droplet was introduced then retracted onto different mineral surfaces including calcite, quartz, muscovite mica (of various levels of purity) and a carbonate-rich caprock sample. They observed significant wettability hysteretic effect and adhesion, particularly on muscovite mica. $\mathrm{H}_{2} \mathrm{~S}$ exhibited wettability reversal from non-wetting, intermediate wetting to highly wetting. The wettability alteration and triple line pinning was partly attributed to surface roughness and defects. The fact that adhesion and contact angle hysteresis happened on molecularly smooth surfaces was recognized and was explained as the irreversible adsorption of the non-wetting phase. They report that adsorption is enhanced with acidic brine resulted from elevated pressure.

Shah et al. noticed large hysteresis increasing with pressure on both V2 and V3 grade (V2 is higher purity and more smooth) muscovite mica cleavages under intermediate pressure, temperature and salinity conditions [2008]. More importantly, they found that liquid $\mathrm{CO}_{2}$ strongly adhered to V2 mica in moderate salinity brine. Under the same condition, quartz showed increasing wettability hysteresis with increasing pressure. In high salinity brine, quartz exhibited large hysteresis and weak $\mathrm{CO}_{2}$ adhesion. They also found that $\mathrm{V} 3$ mica became $\mathrm{H}_{2} \mathrm{~S}$-wetting with strong adhesion to the mineral surface observed in low salinity brine. Tonnet et al., however, 
conducted a similar study and observed low hysteresis and no adhesion in the $\mathrm{V} 2$ mica- $\mathrm{CO}_{2}$ brine system in low salinity brine. They did report $\mathrm{CO}_{2}$ adhesion and increased hysteresis with pressure in high salinity brine with $\mathrm{CO}_{2}$ adhesion appearing above $8 \mathrm{MPa}$ [2010].

For non-clay minerals, represented by silica, Kim et al. observed intermediate wetting behaviors of $\mathrm{CO}_{2}$ droplets in salty brine in a siliceous micromodel at $8.5 \mathrm{MPa}$ and $45^{\circ} \mathrm{C}$ [2012]. Espinoza and Santamarina captured significant wettability alteration of water and brine drops on calcite and quartz in $\mathrm{CO}_{2}$ environment with increasing brine salinity but did not observe significant impact of increasing pressure [Espinoza and Santamarina, 2010]. Tonnet et al. reported moderate hysteresis for calcite- $\mathrm{CO}_{2}$-brine under intermediate pressure and salinity conditions [2010].

The increasing number of reports describing some sort of adhesion and wettability hysteresis at the $\mathrm{CO}_{2}$-brine-mineral interface requires an improved understanding of the mechanisms that are driving these phenomena and the conditions under which they prevail. Buckley et al. investigated the adhesion phenomena of crude oil to certain mineral surfaces and revealed the connection between wettability alteration and adhesion [1989]. Also, they mapped the pH and salinity conditions under which oil adheres to a particular solid surface for several crude oil samples. The results were explained using a combination of electric double layer (EDL) calculations and an ionizable surface group model. Insights were provided on non-adhesion of crude oil caused by water film stabilization as a result of EDL repulsion between oil and mineral. Morrow concluded that reservoir rock mostly maintains a strong affinity for water rather than oil. The wetting properties of a solid are generally dominated by the outermost layer of molecules such as thin liquid films formed during spreading and adsorption [1998]. This is why quartz wettability by oil could be vastly altered by a monolayer of adsorbed water. 
Tripp and Combes conducted infrared spectroscopy to study the weak physisorption of $\mathrm{CO}_{2}$ with surface hydroxyl function groups (silanols) in their spectra region on silica [1998]. It was demonstrated that supercritical $\mathrm{CO}_{2}$ does extract the adsorbed water film from wet silica. McCool and Tripp studied the three main types of silanols, i.e., isolated, hydrogen bonded and inaccessible silanols on silica and further discovered that $\mathrm{CO}_{2}$ penetrates the regions containing inaccessible silanols which originally do not participate in interfacial reactions [2005]. These observations were confirmed with molecular dynamic simulations that $\mathrm{CO}_{2}$ effectively interacts with the hydroxyl groups on mica and silica surfaces via hydrogen bonds [Vishnyakov et al., 2008; Cole et al., 2010]. The wettability hysteresis was then explained as the invading $\mathrm{CO}_{2}$ causes instability to the retreating water film. Their work also indicated that the attraction between $\mathrm{CO}_{2}$ and surface hydroxyls is more prominent when the density of hydroxyls on the surface is high. $\mathrm{N}_{2}$ was predicted to perform similarly to $\mathrm{CO}_{2}$ in terms of interactions with hydroxyls.

Adhesion has been described as a synergetic manifestation of molecular affinity [Kendall, 1994]. The elevated molecular attraction and interfacial energy that results from van der Waals interactions including dispersion (London) forces, electrostatic (Keesom) forces, induction (Debye) forces, hydrogen bonds, solvation forces, hydration forces etc. over short separation distances between surfaces (at the scale of several nanometers) [Kendall, 1994]. Vishnyakov et al.'s simulation showed that the interaction forces between $\mathrm{CO}_{2}$ and silica are only effective within 3 $\mathrm{nm}$ with a minimum distance of $0.5-0.8 \mathrm{~nm}$ [2008].

Derjaguin-Landau-Versey-Overbeek (DLVO) theory has been used to shed light on the dispersion and electrostatic interactions. DLVO theory has been successful applied to $\mathrm{CO}_{2}$-brinemineral mixtures to explore the mechanism controlling the thickness of the thin adsorbed water 
film separating solid and $\mathrm{CO}_{2}$ under GCS relevant conditions [Tokunaga, 2012]. System properties including pressure, temperature, $\mathrm{pH}$ and ionic strength can collectively modify the structure of the nanometer scale water film by affecting the enclosed EDL interactions. van der Waals dispersion and the EDL-pressure relationship was considered in an integrated way and diminished surface charge of silica in the acidified brines caused by $\mathrm{CO}_{2}$ dissolution and increased brine salinity were found to dramatically compress the EDL, in which case dispersion interactions dominates [Tokunaga, 2012].

Adhesion has been shown to be a spontaneous and energetically favorable process [Kendall, 1994]. From the thermodynamic perspective, when adhesion happens, the overall Gibbs free energy in the process decreases, as shown by the Dupré equation in Eq. 5.1.

$$
W=-\Delta G=\gamma_{\text {CO }_{2}-\text { brine }}+\gamma_{\text {brine-solid }}-\gamma_{\mathrm{CO}_{2}-\text { solid }}>0 \quad \text { Eq. } 5.1
$$

Where $W$ is the work of adhesion, i.e., adhesion energy per unit area, which is also the area under the curve of attraction force $P ; \gamma_{\mathrm{CO}_{2}-\text { brine }} ; \gamma_{\text {brine-solid }}$ and $\gamma_{\mathrm{CO}_{2}-\text { solid }}$ are interfacial energy (interfacial tension) between $\mathrm{CO}_{2}$, brine and the solid respectively. Under non-equilibrium conditions, the adhesion energy is represented by its non-equilibrium value É, which can be used in the equilibrium equations, replacing $W$. The adhesion force is linked with adhesion energy (work of adhesion) through Bradley's equation and its derivatives and could also be characterized by the Eyring curve method [Kendall, 1994].

The adhesion energy should be significantly lower when making contact $\left(\mathrm{CO}_{2}\right.$ advancing) than when breaking contact $\left(\mathrm{CO}_{2}\right.$ receding). The adhesion energy is mostly dynamic while equilibrium could occur when change in adhesive energy equals the change in potential energy from the added/subtracted mass and deformation of a $\mathrm{CO}_{2}$ droplet. Work of adhesion acts over very small distances. It is a useful quantity because it distinguishes the two states: contact and 
separation. From an energy balance perspective, the work of adhesion is qualitatively and quantitatively characterized by the reversible work done in the receding contact angle test to pull apart a unit area of the interface.

Adhesion could significantly influence the landscape of $\mathrm{CO}_{2}$ storage and oil recovery. The strong bonding between $\mathrm{CO}_{2} /$ oil and the solids effectively immobilize the fluids. The added trapping capacity could completely dominate the fluctuation in capillary trapping capacity resulted from wettability hysteresis. Even though adhesion has been reported in this system, the conditions under which it occurs are not fully described. In particular, the strength of adhesion, which ultimately determines how much $\mathrm{CO}_{2} /$ oil mass can be immobilized on solid surfaces has not be explicitly studied for $\mathrm{CO}_{2} /$ oil-brine-reservoir systems. This is probably caused by the difficulty associated with carrying out mechanical tests on fluids which have highly non-linear mechanical properties such as poor rigidity and tendency of deformation, making the quantification even harder, especially under high pressure and high temperature conditions.

Efforts have been dedicated to measure the strength of adhesion of mica species in air with an effective application of London dispersion theory [Tabor and Winterton, 1969; Israelachvili and Tabor, 1973]. Adhesion forces can be calculated with the method by Boer [1936] and Hamaker [1937], shown in Eq. 5.2.

$$
P=\frac{-A D}{12 z^{2}} \text { (rigid and stiff body) } \quad \text { Eq. } 5.2
$$

Where $P$ is the adhesion force; $A$ is the Hamaker constant, a macroscopic scaling of the affinity between molecules at the interface; $D$ is the diameter of contacting material, $\mathrm{CO}_{2}$ droplet in this case; and $Z$ is the gap distance between $\mathrm{CO}_{2}$ and solid substrates. The negative sign represents attraction. This equation is valid for contact between rigid bodies and may not be the most ap- 
propriate for understanding $\mathrm{CO}_{2}$ at the surface. But it could be an acceptable first-step estimation of adhesion energy.

Eq. 5.2 suggests that when the distance between the surfaces decreases, adhesive attraction increases sharply. This short-range interaction is more pronounced when the solid surface has smoothness close to its effective range, i.e., nanometer scale. AFM analysis showed that the phlogopite and silica surfaces could be atomically smooth which enables ideal molecular contact. This explained why adhesion favorably happened on phlogopite and silica surfaces rather than the other mineral species from one aspect.

For $\mathrm{CO}_{2}$-brine-mineral system, the non-retarded Hamaker constants for van der Waals interactions with water between $\mathrm{CO}_{2}$ and mineral, $A_{132}$ can be estimated using the combing rule in Eq. 5.3 [Tokunaga, 2012].

$$
A_{132}=\left(\sqrt{A_{11}}-\sqrt{A_{33}}\right)\left(\sqrt{A_{22}}-\sqrt{A_{33}}\right) \quad \text { Eq. } 5.3
$$

where $A_{11}, A_{22}$ and $A_{33}$ are Hamaker constant for pure substances of mineral, $\mathrm{CO}_{2}$ and $\mathrm{H}_{2} \mathrm{O}$, respectively. These Hamaker constants can be calculated using the Lifshitz equation [Israelachvili, 2011] in Eq. 5.4.

$$
A_{i i}=0.75 k_{B} T\left(\frac{\varepsilon_{i}-1}{\varepsilon_{i}+1}\right)^{2}+\frac{3 h_{U_{e}}}{16 \sqrt{2}} \frac{\left(n_{i}^{2}-1\right)^{2}}{\left(n_{i}^{2}+1\right)^{1.5}} \quad \text { Eq. } 5.4
$$

Where $k_{B}$ is the Boltzmann constant; $T$ is temperature in Kelvin; $\varepsilon_{i}$ is the relative permittivity; $h$ is the Planck constant; $U_{e}$ is the primary electronic absorption frequency in ultraviolet region; $n_{i}$ is the refractive index. 
The thickness of adsorbed water film $f$ can then be evaluated with the three phase Hamaker constant $A_{132}$ and the disjoining pressure $\prod_{v d W}$ resulting from van der Waals interactions using Eq. 5.5.

$$
f=\left(\frac{-6 \pi \prod_{v d W}}{A_{132}}\right)^{-1 / 3} \quad \text { Eq. } 5.5
$$

The calculated thickness of water film and Hamaker constant can then be plugged in Eq. 5.2 to calculate the adhesion force.

Molecular-scale adhesion generally depends on the compliance/affinity of the surfaces and is manifested by the smoothness [Broseta et al., 2012]. Surface roughness has been demonstrated to have negligible effects on wettability on homogeneous and well-polished surfaces. However, surface roughness could play an important role in affecting the mobility of $\mathrm{CO}_{2}$ and oil in the reservoir environment. The adhesive energy along with the strength of adhesion, i.e. adhesion force, varies substantially with the molecular interfacial forces, as well as with geometry and material stiffness.

$\mathrm{pH}$ affects adhesion in the way that it permanently restructures the distribution of surface charges and salinity affects adhesion in the way of compressing EDL, which can be quantified by the Debye-Huckel theory [Papirer, 2000]. Many people have reported the long range impact of solid surfaces in the redistribution and rearrangement of the interfacial water network (primarily through Hydrogen bonds that water molecules could be rearranged and reoriented to a specific direction according to the surface charge situation. Yegnaeh et al. [1999] have reported that the water molecules would have their hydrogen atoms oriented towards the negatively charged surface and vice versa for oxygen atoms towards positively charged surface on silica surface. They also reported the strengthening of the hydration layer when the surface charges (positive or nega- 
tive) increase. By using the IR-visible sum frequency generation (SFG), they have demonstrated the increase of the SFG signal at low or high pHs, compared to the mild electrostatic condition (e.g., pHpzc). When extra acid or base was added into the solution, the surface charge of the mineral was dramatically changed. Extra acid would cause accumulation of positive charges and extra base would cause accumulation of negative charges. The increase of charge density on the mineral surface will lead to higher electric field that thickens or compresses the hydration layer between the mineral surface and $\mathrm{CO}_{2}$. Kerisit et al. [2005] reported that the density of water layer is higher than bulk water at the distance of 10-15 angstroms away from the solid surface. This distance is equivalent to multiple layers of water molecule.

No work has been done so far to integrate mechanistic analysis with evaluation of adhesion strength under GCS relevant conditions. The goal of this work is to explore the role that adhesion may be playing in driving contact angle measurements at the $\mathrm{CO}_{2}$-mineral-brine interface and the strength of adhesion that significantly impacts trapping capacity of $\mathrm{CO}_{2}$.

\subsection{Methods}

Adhesion was studied under both equilibrium and dynamic (advancing and receding) conditions under GCS representative conditions (Temperature $<50^{\circ} \mathrm{C}$ and Pressure $<20 \mathrm{MPa}$ ) covering a broad range of $\mathrm{pH}$ and ionic strength in fresh and carbonated synthetic brines. Seven representative minerals including quartz, calcite, amorphous silica, dolomite, kaolinite, illite and phlogopite were selected since these constitute most of the minerals on the pore surfaces in sandstones [Peters, 2009]. These minerals all have hydroxide functional groups, e.g. aluminol (三Al$\mathrm{OH})$ and silanol $(=\mathrm{Si}-\mathrm{OH})$ at the solid surface and are sensitive to the adjacent aqueous phase $\mathrm{pH}$ and ionic strength conditions. $\mathrm{N}_{2}$ control experiments were conducted under identical conditions 
on phlogopite and silica surfaces which exhibited pronounced adhesion. Since surface roughness has impacts on adhesion, extra efforts were devoted to measure wettability hysteresis on the surfaces of kaolinite and illite with different levels of roughness.

Mineral samples were prepared by sectioning high purity rocks, lapping the experimental surface according to the crystal structure with a diamond grinding wheel, and then polishing them with a series of silicon carbide sanding papers down to a roughness ca. $25 \mu \mathrm{m}$ (ca. 50 and $100 \mu \mathrm{m}$ for the samples used in the surface roughness experiments). Before experiments, the parts of equipment and the mineral samples were carefully cleaned following the same protocol in the wettability experiments. Since adhesion is very sensitive process and could be easily disrupted by contaminants at the surface, especially organic compounds, special attention was given during the cleaning process. Because the state of sample surface can be different in dry and wet conditions, all the samples were flushed with at least $200 \mathrm{ml}$ brine solution which is equivalent to 10 times of the vessel volume to fully situate the samples with the brine. The adhesion hysteresis experiments were reproduced at least three times.

To evaluate adhesion of $\mathrm{CO}_{2}$ bubbles on the mineral surface, a modified form of the advancing/receding contact angle measurement was carried out. The conventional way to measure adhesion using solid surfaces is to perform so called "stick-peel-crack" tests where surfaces are allowed to adhere and then an axial tensile force is applied to break the adhesion and this force is proportional to the adhesive surface energy [Kendall, 1994]. In our experiments, "stick and peel" tests were carried out using a modification of the test rig described in Chapter 4, except that the injection needle was extended to approach the sample surface (Figure 5.1). Two pressure control values were positioned in sequence upstream from the needed in order to permit precise control over $\mathrm{CO}_{2}$ droplet flows into and out of the pressure cell. This setup enabled precise control of the 
pressure gradient between the inlet $\mathrm{CO}_{2}$ and the pressure in the vessel. The setup is described in more detail in the Appendix.
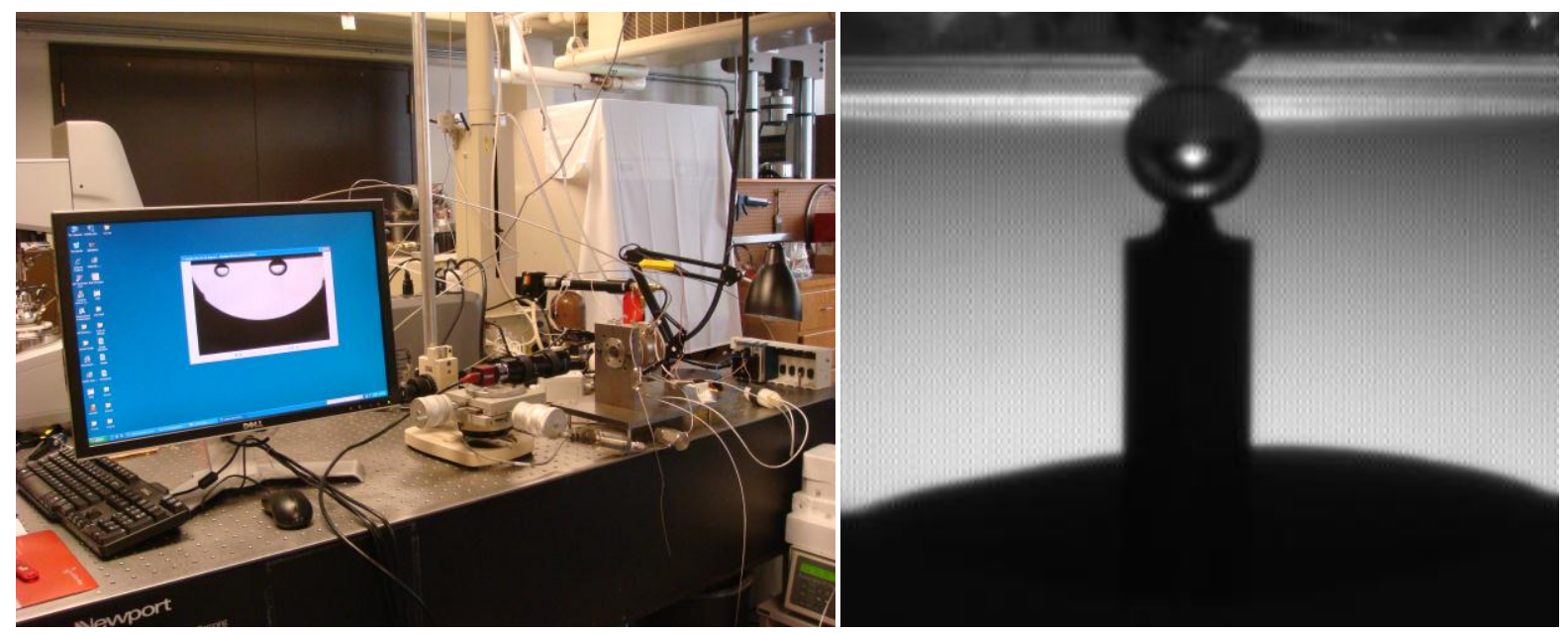

Figure 5.1. Experimental setup. (Left). Contact angle measuring system (Right). Extended needle setup to conduct adhesion hysteresis tests.

\subsection{Results}

On most of the minerals tested here, the CO bubbles showed no particular affinity for the surface. They were water wetting and CO bubbles exhibited contact angles of approximately $20^{\circ}$. Subtle changes to contact angle could be induced by changing the solution chemistry as previously reported [Wang, Edwards et al., 2013]. A very different, and quite interesting, behavior developed for a large number of the bubbles on phlogopite, amorphous silica, and some calcite samples. These bubbles wetted the surface more fully but also adhered to the surface in a way that made them difficult to remove.

The difference between those bubbles that exhibited adhesion and those that did not is shown in Figure 5.2. Adhered bubbles (Figure 5.2b) were much more wetting (larger $\Theta$ ) and there were often differences between the left and right side contact angle of the bubble suggest- 
ing that the triple phase line is not circular. This asymmetric character of adhered bubbles has been reported as a consequence of pinning, which can be caused by adhesion, and by heterogeneities on the surface in terms of chemical composition and surface roughness. Such irregular triple lines are uncommon for bubbles that are generally non-wetting to the surface like those in Figure 5.2a.

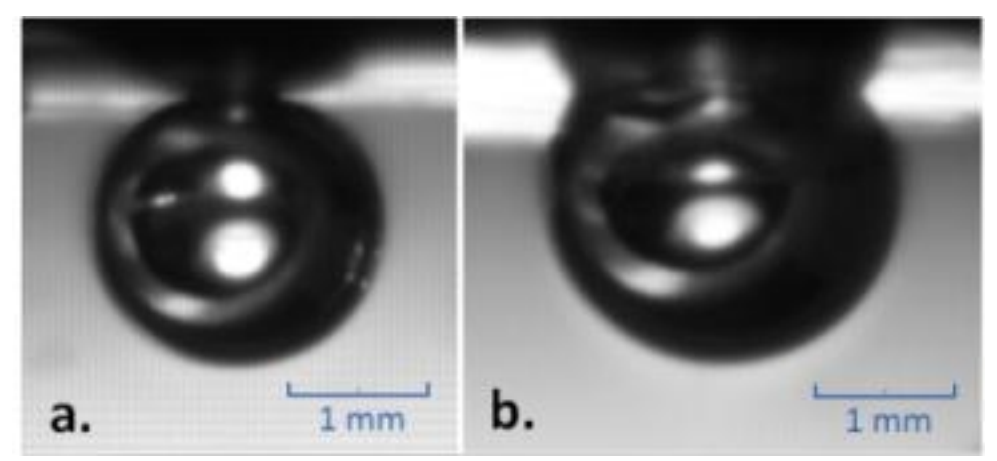

Figure 5.2. Bubble images reveal difference between a. bubbles exhibiting no adhesion $(0.1 \mathrm{M}$ $\left.\mathrm{NaCl}-\mathrm{XCO}_{2}=0\right)$ and $\mathrm{b}$. bubbles exhibiting adhesion $\left(1.5 \mathrm{M} \mathrm{NaCl}-\mathrm{XCO}_{2}=0\right)$ on phlogopite. For the adhesion case, the differences in the contact angle between the left and right side of the bubble are indicative triple line distortion.

Even more striking than changes in contact angle during adhesion are the comparatively strong forces that bound the bubble to the mineral surface (Figure 5.3). Bubbles that did not exhibit adhesion could move readily across the surface, like balls moving across a table. Bubbles that exhibited adhesion were highly resistant to shear forces in the brine (e.g., created using a stir bar), to tilting of the pressure vessel, and to the introduction of other bubbles. Figure 5.3 shows how the creation of a new bubble, which would normally push an existing bubble along the surface, instead lead to displacement of both by the adhered bubble. The contact between the bubble 
and the mineral surface did not change. Eventually, the interfacial tension separating the bubbles was overcome by the adhesive forces and new bubble combined with the bubble at the surface.
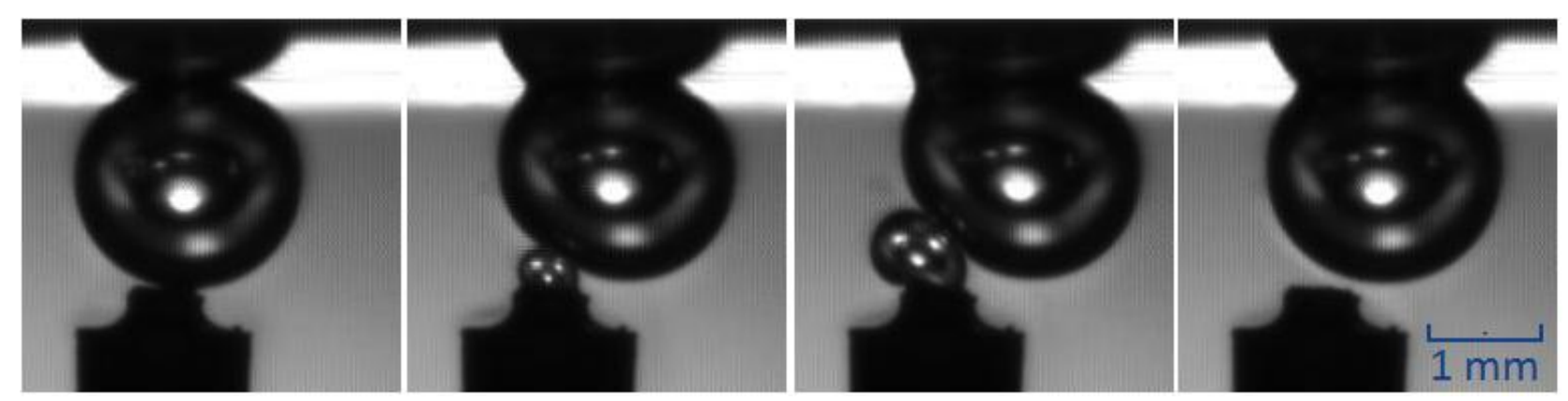

Figure 5.3. Bubbles that adhere to the mineral surface can resist significant shear forces including those created here by introducing a bubble adjacent to the adhered bubble. In these timelapse photos the bubble is introduced on in the second photo from the left, as it grows, the adhered bubble is displaced and eventually the new bubble combines with the existing bubble without movement of the adhered bubble (but an increase in the contact length that corresponds to the greater volume of $\mathrm{CO}_{2}$ in the bubble). (Phlogopite surface $1.5 \mathrm{M} \mathrm{NaCl}-\mathrm{XCO}_{2}=0$ ).

Advancing and receding tests were carried out as a means of understanding the dynamic effect that adhesion has on interfacial behavior. Figure 5.4 shows the progression, left to right, of a bubble being introduced to a phlogopite surface, contacted with the sample, expanded along the surface, then retracted back into the injection needle. The change in the contact angle is evident (and quantified at the bottom of the figure) as the bubble maintains contact with the mineral surface until the surface tension forces overcome the adhesion forces and the bubble separates. As a reference, the bubble on the left in each picture is not adhered to the surface. For bubbles that do no adhere, advancing and receding experiments show no differences between contact angle and there is no distortion in the shape of the bubble as seen in Figure \#\#. 


\section{Contact Phase}

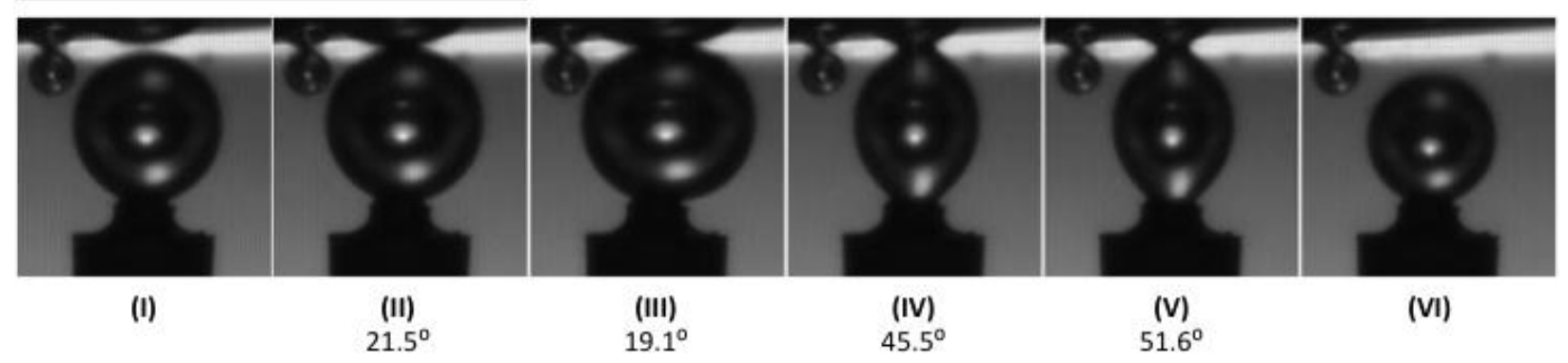

Figure 5.4. Advancing and receding tests of a $\mathrm{CO}_{2}$ bubble exhibiting adhesion on a phlogopite surface. (I-II). the bubble is first contacted to the surface where it grows. At its largest point (III) the bubble has a contact angle that is somewhat higher than a bubble not exhibiting adhesion (shown for reference next to the main bubble). As the bubbles is drawn back into the injection needle (IV-V), the adhesion forces keep the bubble connected to the surface, greatly distorting the contact angle and the shape of the bubble. Eventually (VI) the surface tension forces overcome the adhesion force and the bubble breaks away from the surface.

These types of advancing and receding tests are particularly useful for understanding multiphase flow through porous media and how processes like viscous fingering and snap-off will take place.

One of the most important manifestations of adhesion is that it can impart hysteresis to systems [Chiquet et al., 2007a]. Figure 5.5 plots a number of advancing and receding tests on both phlogopite and silica surfaces. Each dotted line on the plot represents an individual replicate. The advancing contact angles in all examples are highly consistent and comparable to the contact angle for non-adhered bubbles. For mineral surfaces in equilibrium with DI water, brine, and two levels of $\mathrm{CO}_{2}$ partial pressure, the receding contact angle varied considerably and in 
some cases was several times higher than the advancing contact angle. For mineral samples that are in contact with strong acid or base (right two graphs), the advancing and receding contact angles show very little difference.
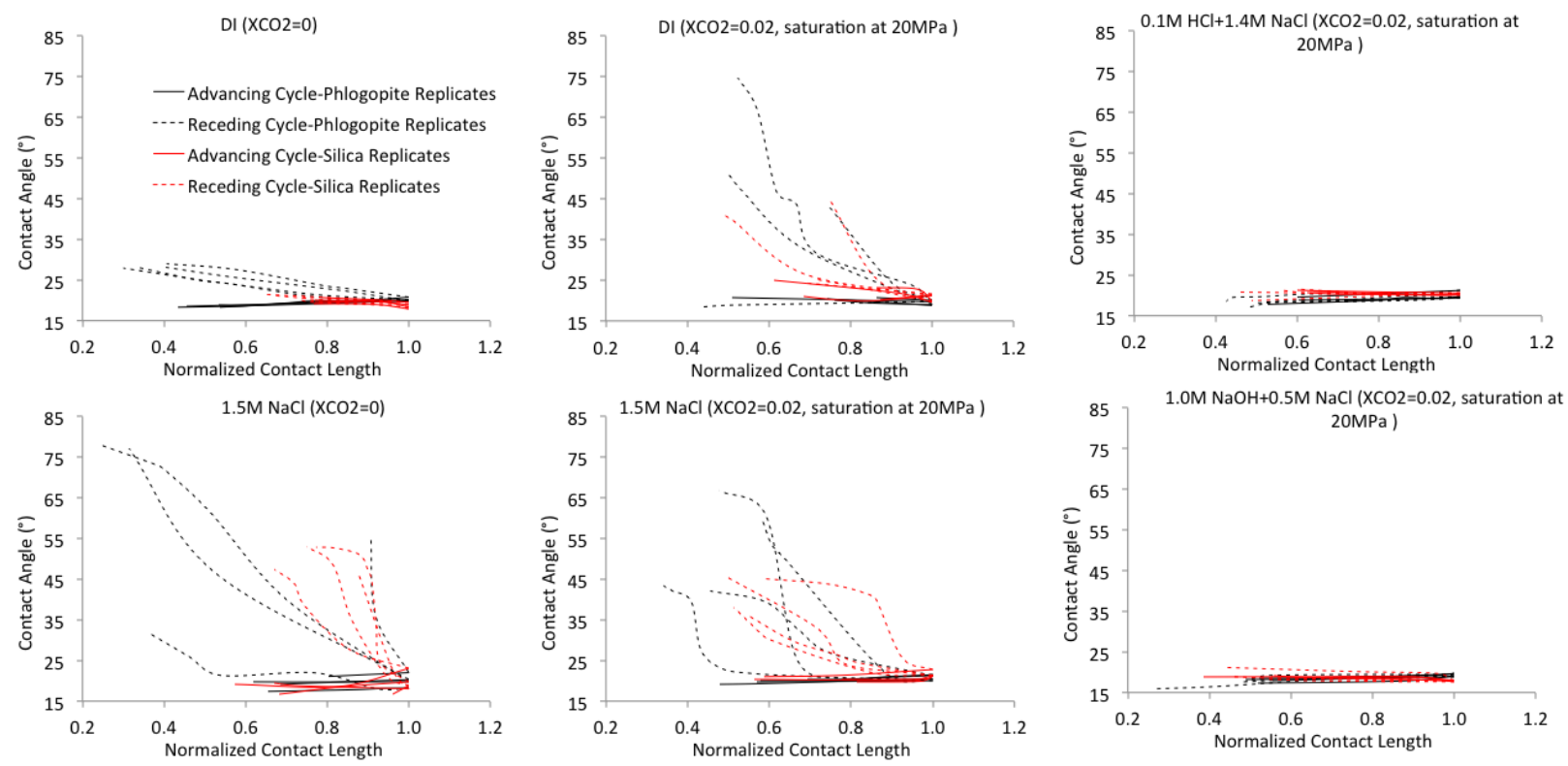

Figure 5.5. Contact angle hysteresis in advancing and receding tests show how adhesion can lead to enhanced wetting on the mineral surface as the bubble is stretched away from the surface.

It should be noted that a kinetic drag effect was evident in these advancing and receding contact angles. Kinetic drag is used to describe processes that move slower because of adhesion forces at the surface. In these experiments, the detachment of the bubble is slowed by the adhesion at the surface relative to a bubble that is not adhered. When mechanical forces, in this case the flow of $\mathrm{CO}_{2}$ onto or off of the mineral surface, disrupt the adhesion at the interface, the separation initially resists the movement and eventually yields with time. Even though time is not indicated in Figure 5.4, our observations in the lab suggest that bubbles adhere to the surface more rapidly than they detach. This is consistent with theories about adhesive drag [Kendall, 1994]. 
The adhesion phenomena observed here can be at least partially understood in term of surface roughness. The two surfaces that exhibited the greatest degree of adhesion were phlogopite and amorphous silica. These were the same two minerals that we did not polish mechanically prior to experiments because their surfaces were understood to already be as smooth if not smoother than what we were able to achieve using our polishing technique. The phlogopite could be easily cleaved into sheets along the basal plane. The amorphous silica used here were from high purity polished glass slides.

In spite of the striking contrast between bubbles exhibiting adhesion and those that are not, the effect was not deterministic with respect to mineral surface and brine conditions. In other words, the frequency with which bubbles adhered to the surface varied considerably (Table 5.1). For a phlogopite surface in DI water, only about $20 \%$ of the bubbles adhered to the surface. These trends were statistically significant and observed over four replicates and hundreds of bubbles. The addition of salt or $\mathrm{CO}_{2}$ increased the incidence of adhesion considerably indicating that brine chemistry was as important as surface roughness in controlling adhesion.

When the phlogopite surface was equilibrated with brine instead of DI water, the percentage of bubbles that adhere increased to $>50 \%$ of the bubbles. Modeling of the electric double layer on the hydroxide mineral interface suggests that an increase in ionic strength will shrink the boundary layer at the surface effectively enabling closer contact between the mineral and the $\mathrm{CO}_{2}$ bubble. Tokunaga developed a computational model for mineral, bine, $\mathrm{CO}_{2}$ systems based on Derjaguin-Landau-Verwey-Overbeck (DLVO) theory and there is a clear and predictable shrinkage of the double layer with increased salinity [2012].

When the partial pressure of $\mathrm{CO}_{2}$ in the brine was increased such that the $\mathrm{CO}_{2}$ bubble and the $\mathrm{CO}_{2}$ in the aqueous phase were in equilibrium, the instance of adhesion increased above the 
levels observed in the brine/mineral system. On these surfaces, about $70-80 \%$ of the bubbles adhered. This is also consistent with electric double layer theory since the carbonate and bicarbonate ions in the solution would interact with the hydroxide surface and neutralize the surface charge, imparting it with higher affinity for non-polar $\mathrm{CO}_{2}$ molecules.

Table 5.1. On a given surface with bubbles exhibiting adhesion there are also bubbles that exhibit no adhesion. The proportion of each can be changed by increasing the salinity of the brine in contact with the phlogopite surface or by increasing the partial pressure of $\mathrm{CO}_{2}$ in the brine.

\begin{tabular}{|c|c|c|}
\hline Brine Solution & Fresh Brine $\left(\chi_{\left.\mathrm{CO}_{2}=0\right)}\right.$ & $\begin{array}{c}\text { Carbonated Brine }\left(\chi_{\mathrm{CO}_{2} \sim 0.02}\right. \\
\text { under 20 MPa saturation })\end{array}$ \\
\hline $\mathrm{DI}$ & $26 \pm 13 \%$ & $19 \pm 11 \%$ \\
\hline $\mathrm{DI}+0.1 \mathrm{M} \mathrm{NaCl}$ & $49 \pm 14 \%$ & $82 \pm 17 \%$ \\
\hline $\mathrm{DI}+0.5 \mathrm{M} \mathrm{NaCl}$ & $58 \pm 25 \%$ & $76 \pm 13 \%$ \\
\hline $\mathrm{DI}+1.0 \mathrm{M} \mathrm{NaCl}$ & $52 \pm 17 \%$ & $71 \pm 23 \%$ \\
\hline $\mathrm{DI}+1.5 \mathrm{M} \mathrm{NaCl}$ & $55 \pm 12 \%$ & $79 \pm 7 \%$ \\
\hline
\end{tabular}

*The frequency is calculated as the ratio of the counted numbers of adhesive and non-adhesive $\mathrm{CO}_{2}$ droplets in each case.

Adhesion has been explained in terms of molecular forces that act on the nanometer scale [Kendall, 1994]. At longer separation distances between surfaces, more traditional electrostatic forces can be used to understand attractive and repulsive forces between surfaces. The adhesion experiments carried out here revealed dramatically different and surprising behavior that could be explained most readily using prevailing theories about adhesion between ultra-smooth surfaces where initial separation distances are on the order of $\sim 10 \mathrm{~nm}$ [Broseta et al., 2012]. Previous studies report a spontaneous jumping together of surfaces to achieve a more metastable adhesive state. This behavior was consistent with our observations. 
Smaller $\mathrm{CO}_{2}$ droplets were preferentially adhesive because adhesion forces decays linearly with droplet size as indicated in the adhesion force equation whereas gravitational force falls following a cubic form as described in the same equation [Kendall, 1994]. The intersection of the two curves provides good estimates of the critical droplet size of adhesion and it is affected by the surface roughness. Also, the adhesion was not a perfectly reversible process where adhesion was mostly not readily reproducible. Right after the adhesion observed in fresh DI water, the pressure vessel was vented and flushed with $\sim 5$ times vessel volume of fresh DI water. After pressure and temperature recovered to the original steady state, supercritical $\mathrm{CO}_{2}$ droplets were immediately introduced to the phlogopite surface again. No adhesion occurred with all the other conditions remaining the same, indicating that the equilibrium of the phlogopite surface energy was interrupted and the equilibrium of adhesion required time to recover to its original steady state. This time-dependency was justified with the adhesive drag and hysteresis phenomena recorded in the adhesion hysteresis experiments.

Moreover, adhesion was an energetically favorable and thus a spontaneous process. Originally non-adhesive $\mathrm{CO}_{2}$ droplets could be easily induced by the existing adhesive ones and transformed to be adhesive with slight interference from outside, forming a larger adhesive droplet. This was because there are multiple levels of meta-stable energy states on the surface of a certain solid species and the energy barrier between these energy states determine the likelihood of the occurrence of adhesion and the transition from non-adhesion to adhesion. Sometimes, a triggering source is needed to initiate adhesion such as pulse, movement, pressure gradient, flow and kinetic energy. Add descriptions of surface defects and salt deposition. Silica exhibited very similar adhesion behaviors as phlogopite and generally has smaller energy barriers for $\mathrm{CO}_{2}$ adhesion compared with phlogopite. 
Figure 5.6 presents a summary of all the contact angle measurements reported here. Eighteen cases are presented to illustrate the trends that emerged from the dozens of cases and hundreds of replicates. As discussed earlier, $\mathrm{CO}_{2}$ bubbles exhibited little adhesion on quartz, kaolinite, calcite and illite. The data marked with the hollow circle represent the average equilibrium contact angle for the droplets showing no adhesion for each set of conditions. The data points were calculated from at least eight randomly selected replicate images. The values of contact angle are very consistent for these bubbles in which adhesion was not observed and so error bars are not included in the figure for clarity. Only subtle changes in contact angle were evident under advancing and receding contact angle conditions.

Much more dramatic changes in wettability were observed for phlogopite and quartz surfaces on average. For these surfaces, a hollow square marker is used to show the mathematical expected value of the equilibrium contact angle for the bubbles exhibiting adhesion. These data points were also calculated with eight stochastically picked replicate images. When adhesion happens, equilibrium contact angle values usually appear in a broad range and these ranges are captured using the error bars included in the figure. These ranges of variation were calculated and presented as the solid lines on the figure, with its half-width being equal to the one-time standard deviation. The blue and red bars represent the minimum and maximum (respectively) observed values for $\mathrm{CO}_{2}$ advancing and receding contact angles. The dashed lines illustrate the range over which observations were recorded for a mineral/brine combination.

Shown in this format, Figure 5.6 illustrates the dramatic difference between cases where adhesion occurs and for those cases in which it does not. When it occurs, the advancing contact angle is similar to the contact angle of the bubbles exhibiting no adhesion. The receding contact angle for an adhered bubble is considerably greater as it resists the force drawing it away from 
the surface. The data in Figure 5.6 also illustrate how thoroughly the adhesion effect is suppressed by the addition of strong acid or base.

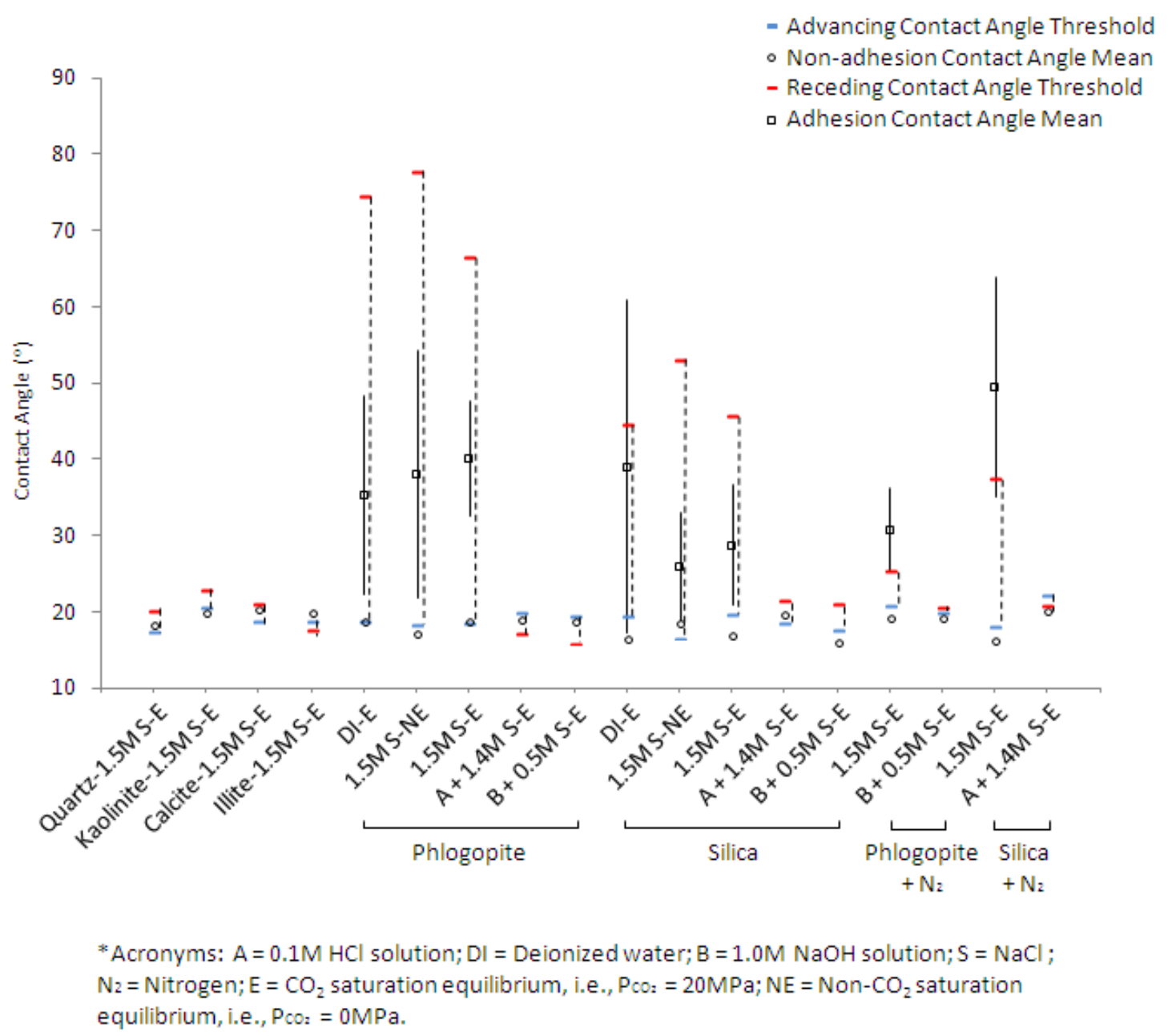

Figure 5.6. Comparison of contact angle measurements shows how dramatically this parameter is impacted by adhesion on the phlogopite and silica surfaces. Similar patterns were also observed for $\mathrm{N}_{2}$ bubbles on these surfaces under pressure.

Many of the same trends were observed when nitrogen was introduced to the surface. Interestingly, $\mathrm{N}_{2}$ exhibited significant adhesion on both phlogopite and silica surfaces but while $\mathrm{CO}_{2}$ had a greater affinity for phlogopite, as measured by the percentage of bubbles that adhere, 
$\mathrm{N}_{2}$ had a relative preference for silica. This trend can be seen in Figure 5.6 where the average contact angle for $\mathrm{N}_{2}$ on silica was higher than the contact angle for any other case tested here. As with the surfaces contacted by $\mathrm{CO}_{2}$, the presence of strong acid or base eliminated the occurrence of adhesion entirely for $\mathrm{N}_{2}$ on hydrated mineral surfaces. This result should not be entirely surprising since $\mathrm{N}_{2}$ is a non-polar molecule somewhat like $\mathrm{CO}_{2} \cdot \mathrm{CO}_{2}$ has a permanent quadrupole moment that sets it apart from $\mathrm{N}_{2}$ in some ways but both $\mathrm{CO}_{2}$ and $\mathrm{N}_{2}$ would be expected to preferentially adhere to mineral surfaces with the lowest amount of net charge.

Additional experiments are needed to resolve the relative important of electrical double layer effects and surface morphology. The electrical double layer is primarily controlled by solution chemistry while the surface roughness controls how closely the gas can come to the mineral surface. Our experiments suggest that adhesion is clearly impacted by both and this will have important implications on the relevance of these findings. For example, $\mathrm{CO}_{2}$ obtained from flue gas could contain trace amounts of sulfur dioxide which, following hydrolysis, would lower $\mathrm{pH}$ and suppress adhesion. As another example, the surface morphology of different diagenetic minerals may suggest that $\mathrm{CO}_{2}$ would undergo hysteretic effects in certain parts where pores walls contain smoother mineral interfaces that lead to adhesion.

The implications of adhesion to multiphase flow through porous media could be significant. In the context of GCS, adhesion could be an important factor for increasing the residual trapping capacity of porous media. Past work suggests that $\mathrm{CO}_{2}(\mathrm{~g})$ will exhibit more adhesion than $\mathrm{CO}_{2}(\mathrm{l})$ or $\mathrm{CO}_{2}(\mathrm{sc})$ further supporting the notion that adhesion could be an important process in controlling $\mathrm{CO}_{2}$ leakage from target formations. Adhesion, and the conditions under which it occurs, are important for site selection and accurate modeling of sequestration capacity in geologic repositories. It could have strong synergies with capillary trapping and mineral trapping. 
This latter process was not explored here but for reactive surfaces, like phlogopite, the intimate contact between the $\mathrm{CO}_{2}$ and the mineral surface could lead to enhanced reaction kinetics as previously suggested [Shao et al., 2011]. 


\section{Chapter 6}

\section{Modeling Vertical Migration of $\mathrm{CO}_{2}$ Through Porous Media}

Improved modeling tools are needed to better understand how likely a parcel of $\mathrm{CO}_{2}$ is to escape and how quickly it will rise. These tools must be based on experimental results collected under GCS-relevant conditions. Here, an improved model of $\mathrm{CO}_{2}$ bubble rise through porous media in the deep subsurface is developed in an effort to better understand leakage processes. As stated in Chapter 2, a parcel of $\mathrm{CO}_{2}$ will be subject to at least three dynamic forces: (1) buoyant forces; (2) surface tension forces; and (3) shear drag forces. To fully characterize these, this work incorporated the experimental measurements reported in Chapters 3 and 4. The viscosity data of brines explored under bubbly flow scenarios are used to better understand the effect of shear drag forces and the rheological conditions that might impact leakage. Wettability data for a range of relevant mineral, brine, $\mathrm{CO}_{2}$ combinations were included to better understand the role of interfacial tension forces on influencing flow. Predicting leakage from geologic carbon sequestration sites is difficult because of the large length scales that are involved and because of the complex geophysics and geochemistry that a rising parcel of $\mathrm{CO}_{2}$ will be subject to as it travels to the surface. The effects and relative importance of five modeling parameters including viscosity of en- 
dogenous brines, contact angle between $\mathrm{CO}_{2}$ and formation minerals, $\mathrm{CO}_{2}$ bubble size, particle size and pore size of porous media are evaluated. The results suggest that each of these parameters is important for controlling leakage and that the priority depends on the characteristics of the formation. Also, the newly reported ganglion model [Wang, Bryan et al., 2013] is validated with seven groups of practical GCS reservoir data to evaluate the critical sizes of ganglion that will result in buoyancy flow. The rise velocity of these $\mathrm{CO}_{2}$ ganglion and its distribution was analyzed using stochastic analysis and sensitivity analysis. At smaller scales, this enhanced model could help explain preferential flow pathways and potential hysteresis that could influence leakage from GCS sites. At larger scales, the results of this work could contribute to more accurate prediction tools for managing the risk associated with GCS.

\subsection{Improved Force Balance Integrating Experimental Data Obtained Here}

$\mathrm{CO}_{2}$ transport through porous media occurs in two scenarios. In the first, $\mathrm{CO}_{2}$ bubble free rise in $\mathrm{CO}_{2}$-saturated brine and small $\mathrm{CO}_{2}$ bubble free rise through coarse porous media without contact with pore walls is considered (Figure 2.1a) and in the second, $\mathrm{CO}_{2}$ channel flow through porous media is modeled (Figure 2.1b).

\subsubsection{Modeling Approach}

In a broad sense, leakage processes of interest for GCS applications involve all those in which $\mathrm{CO}_{2}$ escapes from the reservoirs into which it is originally injected. To understand these pathways, it is first useful to consider the mechanisms by which $\mathrm{CO}_{2}$ could become trapped in the subsurface. These trapping processes have been much better studied. After injection, super- 
critical/liquid $\mathrm{CO}_{2}$ will stay on top of connate brine as a discrete phase due to buoyancy and it will be trapped underneath the caprock. This is known as structural trapping and will prevent immediate buoyant rise of the $\mathrm{CO}_{2}$ while the slower trapping mechanisms commence. $\mathrm{CO}_{2}$ will dissolve into brine and reach equilibrium that is a function of pressure and temperature. Precipitation of the dissolved carbonate materials with cation species in the formation brines will constitute permanent mineral trapping. Similarly, when the $\mathrm{CO}_{2}$ reaches dead end pore spaces in the porous media, capillary trapping will occur that can persist over long length scales [Bachu, 2008; Mansoori et al., 2009].

In cases where these permanent or semi-permanent trapping conditions do not exist, $\mathrm{CO}_{2}$ or $\mathrm{CO}_{2}$-saturated brines can rise through the subsurface in response to buoyant forces. For the $\mathrm{CO}_{2}$-saturated brines, the $\mathrm{CO}_{2}$ will move via advection, diffusion and dispersion. As it moves to lower pressure and temperature formations closer to the surface, the $\mathrm{CO}_{2}$ will become increasingly insoluble and this lead to the ebullition of $\mathrm{CO}_{2}$ bubbles. The pressure within the bubble, described by Henry's Law [Crandell et al., 2010] must exceed the sum of the hydrostatic pressure and the interfacial tension of brine which must be overcome to form the bubble, which can be expressed as:

$$
P=\sum_{i} C_{i} H_{i}>P_{y}+P_{s t} \quad \text { Eq. } 6.1
$$

where the pressure inside the bubble $P$ is equal to the sum of the partial pressures of the volatile species, $C_{i}$ is the aqueous concentration of the volatile species i, $H_{i}$ is the Henry's law coefficients for each species $\mathrm{i}, P_{y}$ is the hydrostatic pressure at depth $\mathrm{y}$, and $P_{s t}$ is the interfacial tension pressure. Once a bubble exists it will rise in response to buoyant forces and its velocity can be determined whether or not it is in contact with the pore walls. 


\subsubsection{1 $\mathrm{CO}_{2}$ Bubble Rise}

Gaseous $\mathrm{CO}_{2}$ experiences free rise when small bubbles rise upwards without contacting the wall of the pores, i.e. $\left(\mathrm{R}_{\mathrm{CO}_{2}}<\mathrm{R}_{\mathrm{P}}\right)$. For this simple case, the rise is driven by buoyancy force and retarded solely by drag force. Under low Reynolds's number conditions, the rise velocity of $\mathrm{CO}_{2}$ can be described by Stokes Law, shown in Equation 3.5 [Poletto and Joseph, 1995]. Low Reynolds number are generally those on the order of one, i.e., $\boldsymbol{R}_{e} \sim 1$, and this typically occurs when the $\mathrm{CO}_{2}$ bubble diameter is less than $1 \mathrm{~mm}$. The size of the bubble is also important because small bubbles can be treated as rigid bodies, i.e., there is no deformation and the bubbles have a spherical geometry [McGinnis et al., 2006].

Viscosity is one of the most important thermophysical properties associated with $\mathrm{CO}_{2}$ bubble rise since it directly impacts the drag force. Existing work has largely relied on the viscosity of pure water to approximate the characteristics of $\mathrm{CO}_{2}$-saturated brine [Oldenburg and Lewicki, 2006]. To improve accuracy of calculation of $\mathrm{CO}_{2}$ rise velocity by Stokes Law, condition specific viscosity from experimental measurements need to be used. Viscosity of $\mathrm{CO}_{2-}$ brine mixtures under a variety of temperature, pressure and salinity conditions were included in the model.

\subsubsection{2 $\mathrm{CO}_{2}$ Channel Flow through Porous Media}

Stokes law is useful for cases in which the bubble does not contact the walls of pores through which it is moving. Most of the $\mathrm{CO}_{2}$ in a leaking parcel will contact the pore walls effectively creating channel flow conditions. For this case of buoyancy-driven channel flow of gases through flooded porous media, other models will be needed, and several have been proposed and explored in the literature. The modeling framework used here is based on the work Corapcioglu 
et al. who derived a theoretical model to describe the rise of air through a porous medium based on a macroscopic force balance acting $\mathrm{CO}_{2}$ [Corapcioglu et al., 2004].

When these assumptions are met, a force balance on a rising bubble in contact with the pore walls forms the basis for an analytical model of channel flow [Ergun, 1952]. The governing equation incorporates inertial force, added mass force, buoyant force, interfacial tension and drag force that results from the momentum transfer between different phases (Eq. 3.11). Under steady state, the equilibrium rise velocity can be calculated using Eq. 3.12.

\subsubsection{Conditions Considered Here}

To describe leakage processes over a range of relevant conditions, two P-T paths were chosen as the basis for the experiments and simulations reported here (Table 6.1). The first represents near surface conditions whereby $\mathrm{CO}_{2}$ would begin at $40^{\circ} \mathrm{C}$ and $4 \mathrm{MPa}$ and then rise to a point where the conditions are at $30^{\circ} \mathrm{C}$ and $1 \mathrm{MPa}$. This first condition corresponds to an average surface temperature of $26^{\circ} \mathrm{C}$ and a linear thermal gradient of $33.3^{\circ} \mathrm{C} / \mathrm{km}$. The second would be representative of a more significant leakage event with the $\mathrm{CO}_{2}$ originating under conditions corresponding to deeper formations, $50^{\circ} \mathrm{C}$ and $20 \mathrm{MPa}$, and subsequently rising to a shallower formation with conditions of $30^{\circ} \mathrm{C}$ and $7 \mathrm{MPa}$. This corresponds to a surface temperature of $19^{\circ} \mathrm{C}$ and a linear thermal gradient of $15.4^{\circ} \mathrm{C} / \mathrm{km}$. 
Table 6.1. Leakage paths investigated in this study

\begin{tabular}{c|cccc|cc}
\hline Leakage & $\begin{array}{c}\text { Temperature } \\
\text { Path }\end{array}$ & $\begin{array}{c}\text { Pressure } \\
\left({ }^{\circ} \mathrm{C}\right)\end{array}$ & $\begin{array}{c}\text { Depth } \\
(\mathrm{MPa})\end{array}$ & $\begin{array}{c}\mathrm{km}) \\
\text { Phase State }\end{array}$ & $\begin{array}{c}\text { Surface Tempera- } \\
\text { ture }\left({ }^{\circ} \mathrm{C}\right)\end{array}$ & $\begin{array}{c}\text { Thermal } \\
\text { Gradient } \\
\left({ }^{\circ} \mathrm{C} / \mathrm{km}\right)\end{array}$ \\
\hline 1 & 30 & 1 & 0.1 & Gas & 26.7 & 33.3 \\
\hline 2 & 40 & 4 & 0.4 & Gas & & 15.4 \\
\hline
\end{tabular}

To improve existing modeling frameworks for evaluating bubble rise through porous media, a detailed sensitivity analysis was carried out on the Corapcioglu model and an improved $F_{s t}$ term was used based on updated contact angle results collected in our laboratory. The sensitivity analysis was carried out to evaluate the relative importance of five key model inputs, namely the effective porosity $\phi$, grain size $d_{p}$, contact angle between $\mathrm{CO}_{2}$ and mineral surfaces $\theta$, bubble size $R_{\mathrm{CO}_{2}}$ and pore size $R_{p}$ on affecting the rise velocity of $\mathrm{CO}_{2}$. The two cases described in Table 6.1 (i.e., P-T conditions corresponding to $30{ }^{\circ} \mathrm{C}$ and $7 \mathrm{MPa}, 50{ }^{\circ} \mathrm{C}$ and $20 \mathrm{MPa}$ ) are investigated. The corresponding thermophysical property parameters used are listed in Table 6.2. For the five experimental inputs of interest, statistical ranges were defined and these are presented in Table 6.3. The values for Case 1 were selected to approximate the properties of Berea sandstone and the values for Case 2 were selected to represent particles of relatively uniform and larger grain size packed in the pattern of orthorhombic arrangement as in [Corapcioglu et al., 2004]. The Berea sandstone case represents the scenario of relatively small porosity, pore size, matrix grain size and bubble radius while Case 2 bounds the conditions at the other end. A few different 
types of distribution for these five variables including normal, log-normal, gamma and Weibull were tested to fit the data and the results indicate that log-normal generally has a superior fit to all the variables. The statistical characteristics are regressed with respect to lognormal distribution using Microsoft ${ }^{\circledR}$ Excel "Solver" add-in. A Monte-Carlo analysis on the stochastic sensitivity of respective variables under different conditions was carried out using these distributions of parameters as model inputs using the Oracle ${ }^{\circledR}$ Crystal ball.

Table 6.2. Model parameter used in this work

\begin{tabular}{|c|c|c|c|c|}
\hline Parameters & Units & \multicolumn{2}{|c|}{ Values } & References \\
\hline gravitational acceleration, $g$ & $\mathrm{~m} / \mathrm{S}^{2}$ & \multicolumn{2}{|c|}{9.81} & \\
\hline density of brine, $\rho_{\text {matrix }}$ & $\mathrm{kg} / \mathrm{m}^{3}$ & \multicolumn{2}{|c|}{1020} & \\
\hline Correction factor, $A$ & \multirow{4}{*}{$\mathrm{N} \cdot \mathrm{m}$} & \multicolumn{2}{|c|}{26.8} & [Corapcioglu et al. 2004] \\
\hline \multirow{3}{*}{ Surface tension, $\sigma$} & & \multirow{2}{*}{\multicolumn{2}{|c|}{0.04}} & [Chiquet et al. 2007b; Espinoza \\
\hline & & & & \\
\hline & & Low & High & \\
\hline Temperature, $T$ & ${ }^{\circ} \mathrm{C}$ & 30 & 50 & \\
\hline Pressure, $P$ & $\mathrm{MPa}$ & 7 & 20 & \\
\hline Depth & $\mathrm{m}$ & 700 & 2000 & \\
\hline Ionic Strength, $S$ & $\mathrm{~mol} / \mathrm{L}$ & $7.1 \sim 7.6 \times 10^{-4}$ & 0.65 & \\
\hline viscosity of $\mathrm{CO}_{2}, \mu_{\mathrm{CO}_{2}}$ & $\mathrm{~Pa} \cdot \mathrm{S}$ & $2.14 \times 10^{-5}$ & $6.87 \times 10^{-5}$ & [Wang and Clarens 2012] \\
\hline density of $\mathrm{CO}_{2}, \rho_{\mathrm{CO}_{2}}$ & $\mathrm{~kg} / \mathrm{m}^{3}$ & 266.56 & 784.29 & [Span et al. 2000] \\
\hline
\end{tabular}


Table 6.3. Model parameters used in Oracle ${ }^{\circledR}$ Crystal Ball. All parameters are fit to lognormal distributions.

Case $1 \quad$ Case 2

\begin{tabular}{|c|c|c|c|c|c|}
\hline Parameter & $\bar{x}$ & $\sigma$ & $\bar{x}$ & $\sigma$ & Reference \\
\hline Effective Porosity (\%) & 0.2386 & 0.056 & 0.3954 & 0.2 & $\begin{array}{l}\text { [Widjajakusuma et al. 1999; } \\
\text { Song 2001; Chen et al. 2003; } \\
\text { Padhy et al. 2007] }\end{array}$ \\
\hline Grain Size (m) & 0.00025 & 0.00008 & 0.004 & 0.003 & $\begin{array}{c}\text { [Peters 2009; Glover and } \\
\text { Walker 2011] }\end{array}$ \\
\hline Pore Throat (m) & 0.00001 & 0.00000254 & 0.0001 & 0.00005 & $\begin{array}{c}\text { [Song 2001; Padhy et al. } \\
\text { 2007] }\end{array}$ \\
\hline Bubble Radius (m) & 0.0005 & 0.0003 & 0.002 & 0.001 & $\begin{array}{l}\text { Experimental data from this } \\
\text { work }\end{array}$ \\
\hline
\end{tabular}




\subsubsection{Results}

\subsubsection{1 $\mathrm{CO}_{2}$ Bubble Rise}

Dissolved $\mathrm{CO}_{2}$ has been shown to increase the viscosity of brines and this behavior is consistent with other acidic solutions that exhibit higher viscosity than water alone [Bando et al., 2004]. The magnitude of this viscosity increase is typically small $(<20 \%)$. A much more significant increase in the effective viscosity of the solution was observed after phase separation when the presence of bubbles increased the shear drag force. Bubbles resist deformation and this effect can increase the viscosity of the brine by $300-400 \%$. At higher temperatures and higher ionic strength, the effect is less pronounced. The full results are reported in Wang and Clarens [2012]. The results of velocity calculated using Stokes' Law have been shown in Figure 3.12.

\subsubsection{2 $\mathrm{CO}_{2}$ Free Rise through Porous Media}

Most models of bubble rise through porous media have assumed a constant value for contact angle $\left(150^{\circ}\right)$ [Oldenburg and Lewicki 2006; Cihan and Corapcioglu 2008]. In reality this value varies as a function of the composition of the host formation. Peters reported that even though digenetic clay minerals such as Kaolinite may only account for 5\% to $31 \%$ of the formation by mass, they account for much more of the pore-mineral contact boundaries, typically in the range of $65 \%$ to $86 \%$ [Peters, 2009]. This suggests that more detailed data on interfacial properties are needed for developing accurate models of bubble rise. This same study found that kaolin is responsible for $75 \%$ of pore-mineral contact boundaries, quartz for $17 \%$, feldspar for $5 \%$ and calcite for $3 \%$. Contact angle measurements for the solids were carried out here and the results are presented in Table 6.4 [Wang, Edwards et al., 2013]. An effective contact angle term 
is defined that is a weighted average of the contact angles and the percentage distributions measured by Peters. The distribution characteristic numbers are regressed and summarized in Table 6.5.

Table 6.4. Results of contact angle measured in this work

\begin{tabular}{|c|c|c|c|c|}
\hline & & & \multicolumn{2}{|c|}{ Ionic Strength (M) } \\
\hline \multirow{2}{*}{ Mineral } & \multirow{2}{*}{ Temperature $\left({ }^{\circ} \mathrm{C}\right)$} & \multirow{2}{*}{ Pressure (MPa) } & $7.1 \sim 7.6 \times 10^{-4} \mathrm{M}$ & $0.65 \mathrm{M}$ \\
\hline & & & Contact an & $\left({ }^{\circ}\right)$ \\
\hline \multirow{2}{*}{ Kaolin } & 30 & 7 & 158.2 & 155.9 \\
\hline & 50 & 20 & 161.3 & 154.5 \\
\hline \multirow{2}{*}{ Quartz } & 30 & 7 & 151.3 & 154.8 \\
\hline & 50 & 20 & 152.5 & 156.5 \\
\hline \multirow{2}{*}{ Feldspar } & 30 & 7 & 150.9 & 161.7 \\
\hline & 50 & 20 & 161.1 & 160.4 \\
\hline \multirow{2}{*}{ Calcite } & 30 & 7 & 143.1 & 151.0 \\
\hline & 50 & 20 & 151.2 & 146.6 \\
\hline
\end{tabular}


Table 6.5. Statistical distribution of contact angle

\begin{tabular}{cccc|cc}
\hline \multicolumn{5}{c|}{ Experimental Conditions } & \multicolumn{2}{c}{ Log-Normal Distribution } \\
\hline Temperature & Pressure & Depth $(\mathrm{m})$ & Ionic Strength & Mean & Standard Devia- \\
$\left({ }^{\circ} \mathrm{C}\right)$ & $(\mathrm{MPa})$ & & $(\mathrm{M})$ & & tion \\
\hline 30 & 7 & 700 & $7.1 \sim 7.6 \times 10^{-4} \mathrm{M}$ & 156.2 & 13.4 \\
30 & 7 & 700 & $0.65 \mathrm{M}$ & 155.9 & 19.5 \\
50 & 20 & 2000 & $7.1 \sim 7.6 \times 10^{-4} \mathrm{M}$ & 159.5 & 23.7 \\
50 & 20 & 2000 & $0.65 \mathrm{M}$ & 154.9 & 17.9 \\
\hline
\end{tabular}

Using the two groups of distribution characteristics (Case 1 and 2) for impact factors presented in Table 6.1 and the measured contact angle data, the $\mathrm{CO}_{2}$ rise velocity through porous media was estimated (Table 6.6). These modeling results demonstrate the important effect that formation characteristics can have on bubble rise velocity with the $\mathrm{CO}_{2}$ rising through the Case 2 formation moving much faster than through the Case 1 formation. The full distributions of the bubble rise velocity as estimated using crystal ball are shown in Figure 6.1. As expected, the velocity of the bubble increases near the surface. Given how much slower the $\mathrm{CO}_{2}$ leaks from deep formations, it strongly supports efforts to put $\mathrm{CO}_{2}$ into the deepest formations that are economically viable. Using the mean linear velocities estimated here, assuming that velocity would increase according to a linear interpolation, and that a parcel of $\mathrm{CO}_{2}$ was not trapped (e.g., it had an unobstructed hydraulic path to the surface), a formation described in Case 1 would leak in 240 days while a formation described by Case 2 would leak in 1.35 days. 
Table 6.6. Distribution of rise velocity of $\mathrm{CO}_{2}$ bubble flow

\begin{tabular}{cccc|cc}
\hline \multicolumn{3}{c|}{ Experimental Conditions } & \multicolumn{2}{l}{ Rise Velocity Distribution (m/s) } \\
\hline Temperature $\left({ }^{\circ} \mathrm{C}\right)$ & Pressure (MPa) & Depth $(\mathrm{m})$ & Case & Mean & Standard Deviation \\
\hline 30 & 7 & 700 & 1 & 0.000085 & 0.00081 \\
& & 2 & 0.0175 & 0.0413 \\
\hline 50 & 20 & 2000 & 1 & 0.00004 & 0.00049 \\
& & 2 & 0.0048 & 0.011 \\
\hline
\end{tabular}
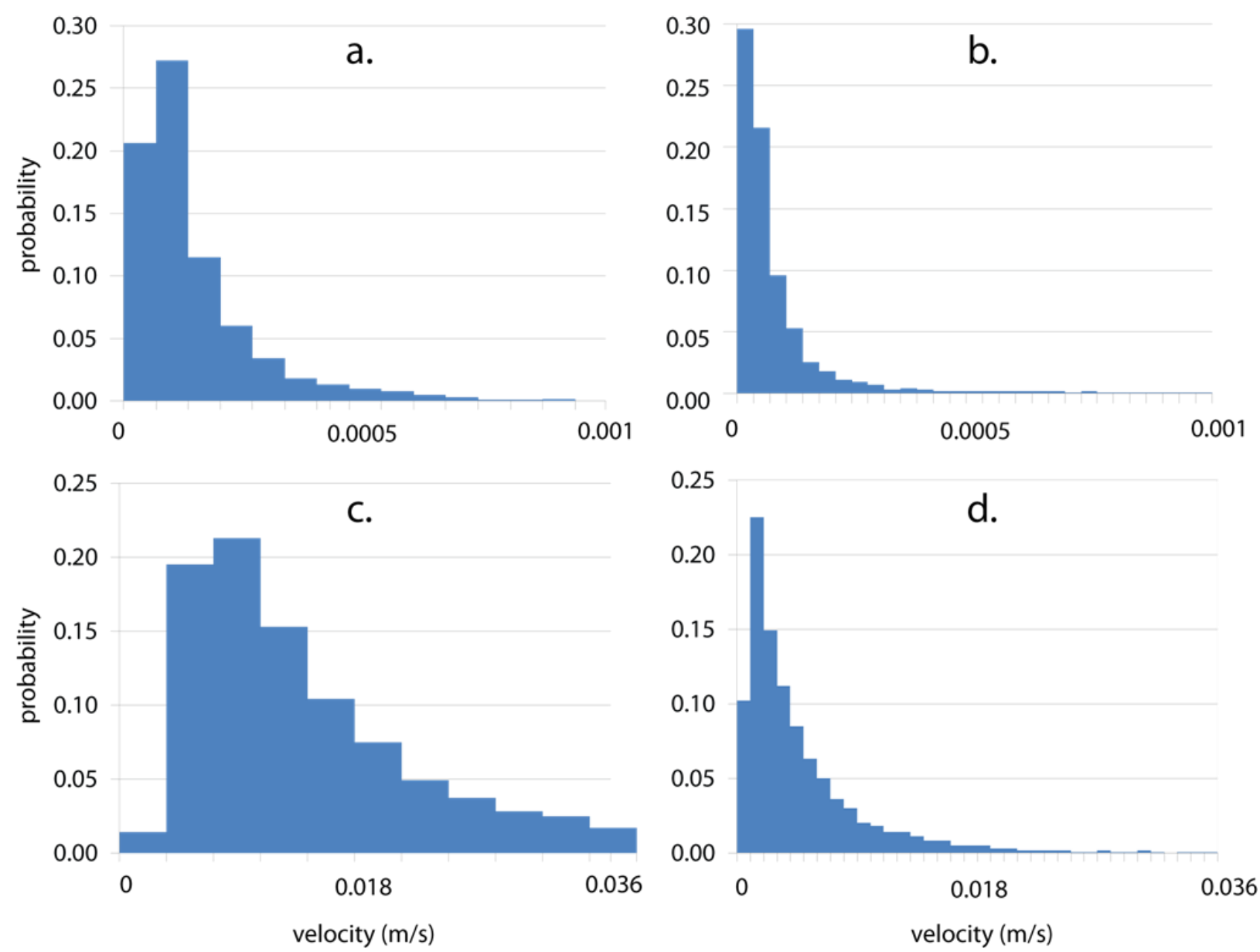

Figure 6.1. Distributions of bubble rise velocity estimate. Case 1: (a) $30^{\circ} \mathrm{C}, 7 \mathrm{MPa}$; (b) $50^{\circ} \mathrm{C}, 20$ $\mathrm{MPa}$; Case 2: (c) $30^{\circ} \mathrm{C}, 7 \mathrm{MPa}$; (d) $50^{\circ} \mathrm{C}, 20 \mathrm{MPa}$. 
The sensitivity of the bubble rise through porous media model was assessed and the results are shown in Figure 6.2. These tornado plots illustrate the top 5 input parameters (contact angle, $\mathrm{CO}_{2}$ bubble radius, effective porosity, grain size and pore size of porous media) to impact the velocity estimates of the model. These are calculated by varying each input parameter in turn via a $\pm 10 \%$ change in the magnitude of the parameters. The centerline in these plots represents the baseline case as reported in Table 6.6. The light and dark-shaded bars imply direct or inverse relationships, respectively. In other words, velocity is positively correlated with some variables (contact angle, $\mathrm{CO}_{2}$ bubble radius, effective porosity and grain size) and negatively correlated with others (pore throat). The results in Figure 6.2a correspond to the Case 1 condition (i.e., porous media with smaller effective porosity, grain and pore sizes and $\mathrm{CO}_{2}$ bubble radius). Under these conditions, contact angle has the highest impact on the terminal rise velocity of $\mathrm{CO}_{2}$, followed by $\mathrm{CO}_{2}$ bubble radius, effective porosity, grain size and pore size of porous media. To illustrate how big of an effect contact angle is under these conditions, it is useful to consider that a $10 \%$ increase in contact angle results in an increase from $1.66 \times 10^{-5}$ to $7.98 \times 10^{-5} \mathrm{~m} / \mathrm{s}$ in $\mathrm{CO}_{2}$ rise velocity (a 400\% increase). Meanwhile, a 10\% increase in pore throat results in a decrease from $5.18 \times 10^{-5}$ to $4.06 \times 10^{-5} \mathrm{~m} / \mathrm{s}$ in $\mathrm{CO}_{2}$ rise velocity (a $22 \%$ decrease). For equivalent formation conditions, the importance of these model inputs seems to remain constant. The results under Case 2 conditions (Figure 6.2b), which represents the scenario of relatively large values of effective porosity, grain and pore sizes and $\mathrm{CO}_{2}$ bubble radius, the sensitivity is somewhat different with effective porosity being most important followed by grain size, contact angle, bubble radius and pore throat. These results are consistent with more anecdotal observations of related systems. For example, Corapcioglu observed that surface tension forces, which are directly proportional to contact angle have the most significant effect on smaller bubbles [Corapcioglu et al., 2004]. 
The effect of wetting vs. nonwetting behavior on mineral surfaces was considered in the context of the sensitivity analysis. Because of the sinusoidal function that includes contact angle, the net effect on vertical bubble velocity is the same for wetting and nonwetting surfaces (because $\sin \theta=\sin (\pi-\theta)$ ). When contact angles are complementary, nonwettability, i.e. large contact angle is much more favorable than wettability because the capillary pressure resulting from larger contact angles could have greater capacity for capillary trapping thereby providing the time for other, permanent sequestration processes to occur such as mineralization or dissolution [Chiquet et al., 2007a].

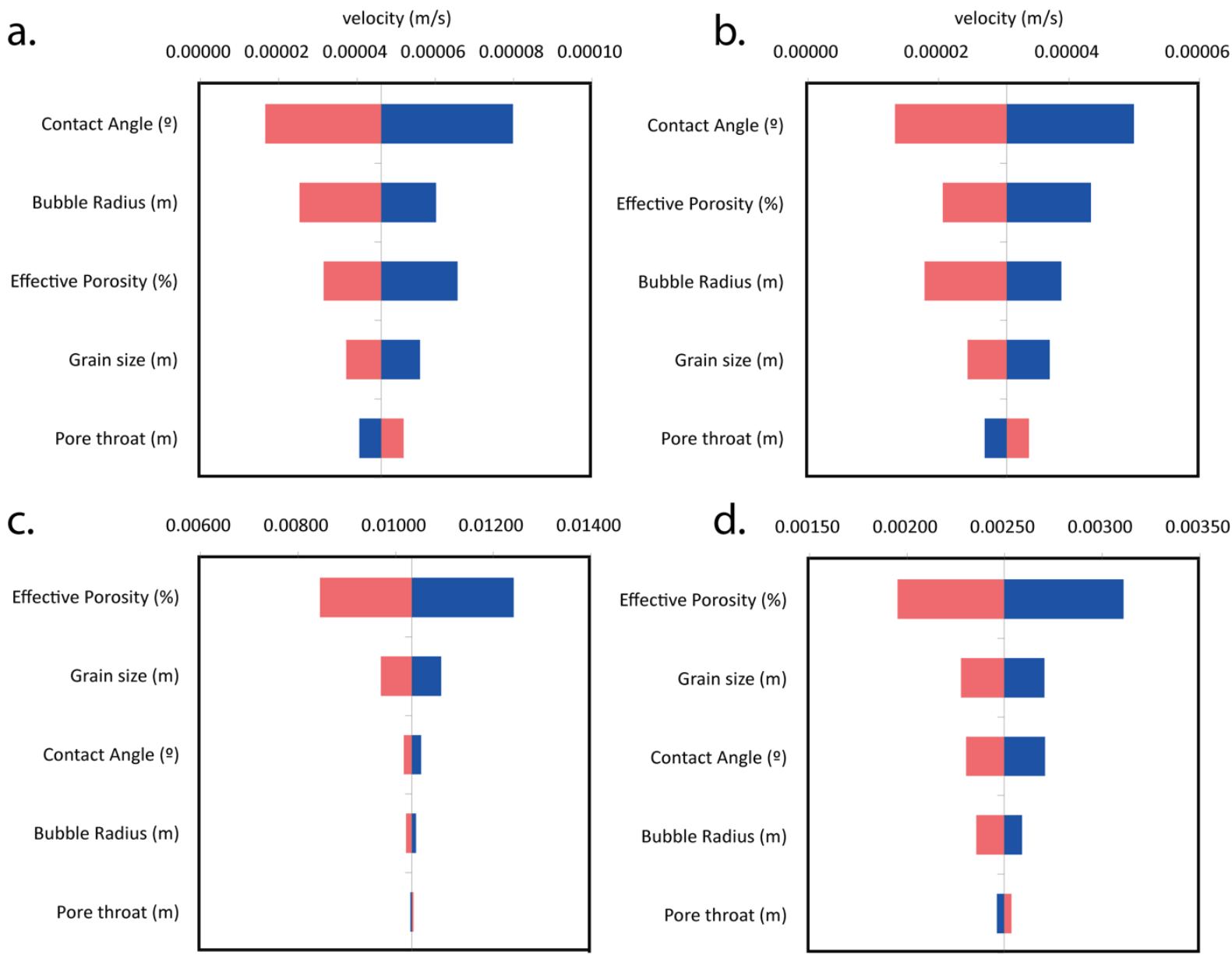

Figure 6.2. Tornado plots manifest to what extent $\mathrm{CO}_{2}$ rise velocity is sensitive to a $\pm 10 \%$ change in input parameters including contact angle, $\mathrm{CO}_{2}$ bubble radius, effective porosity, grain size and pore size of porous media. Case 1: (a) $30^{\circ} \mathrm{C}, 7 \mathrm{MPa}$; (b) $50^{\circ} \mathrm{C}, 20 \mathrm{MPa}$; Case 2: (c) $30^{\circ} \mathrm{C}, 7 \mathrm{MPa}$; (d) $50^{\circ} \mathrm{C}, 20 \mathrm{MPa}$. 


\subsection{Ganglion Model Simulation Using Reservoir Data}

Wang and Bryan et al. recently developed a theoretical model based on the assumptions of Darcy's flow and incompressible flow of $\mathrm{CO}_{2}$ [2013]. This model described the entrapment condition of $\mathrm{a} \mathrm{CO}_{2}$ ganglion of certain size:

$$
H<H_{c}=\frac{2 \sigma \cos \theta}{\Delta \rho g}\left(\frac{1}{r_{n}}-\frac{1}{r_{p}}\right) \quad \text { Eq.6.2 }
$$

where $H$ is the ganglion size, $H_{c}$ is the critical ganglion size, above which $\mathrm{CO}_{2}$ ganglion can turn into a buoyancy-driven flow; $\sigma$ is the interfacial tension between $\mathrm{CO}_{2}$ and brine; $\theta$ is contact angle between $\mathrm{CO}_{2}$, brine and reservoir mineral; $\Delta \rho$ is the density difference between $\mathrm{CO}_{2}$ and brine; $g$ is the gravitational constant; $\boldsymbol{r}_{n}$ and $\boldsymbol{r}_{p}$ are the effective radii of pore throat and pore body, respectively. When the ganglion size $H$ is smaller than the critical ganglion size $H_{c}$, $\mathrm{CO}_{2}$ stays trapped by capillary forces. Once the critical ganglion size is overcome, upwarddriven buoyancy overpowers capillary pressure and a $\mathrm{CO}_{2}$ ganglion could possibly move through porous media.

A closed formed equation to calculate the rise velocity of $\mathrm{CO}_{2}$ ganglion is presented in Eq. 6.3 [Wang and Bryan et al., 2013].

$$
V=\frac{k}{\mu_{W}+\mu_{\mathrm{CO}_{2}}}\left[\Delta \rho g-\frac{2 \sigma \cos \theta}{H}\left(\frac{1}{r_{n}}-\frac{1}{r_{p}}\right)\right] \quad \text { Eq. } 6.3
$$

where $\mu_{W}, \mu_{\mathrm{CO}_{2}}$ are the viscosity of brine and $\mathrm{CO}_{2}$, respectively; $k$ is the permeability of a particular medium. This equation incorporates several important geophysical and thermophysical properties of a specific geologic porous medium. 
The capacity of a specific geologic medium for capillary trapping (CCT) can then be estimated by Eq.6.4 [Wang and Bryan et al., 2013].

$$
C C T \propto \int_{r_{p}}^{H_{c}} p(L) d L=\frac{1}{r_{p}}-\frac{\Delta \rho g}{2 \sigma \cos \theta} \frac{r_{p} r_{n}}{r_{p}-r_{n}} \quad \text { Eq.6.4 }
$$

where $p(L)$ is the number of ganglion of size $L$.

GCS reservoir data have been found to evaluate the performance of this ganglion model. Geologic data of six reservoirs in the lower cretaceous Fall River formation in South Dakota and Wyoming including three in Red Canyon and Buffalo Basin Outcrops and three in Buck Draw field, were used [Dutton and Willis, 1998]. The distribution of pore body, pore throat, porosity and permeability in the six selected reservoirs are summarized in Table 6.8, where the reservoirs are presented with permeability from high to low. Representative reservoir conditions: $20 \mathrm{MPa}$ and $50^{\circ} \mathrm{C}$ are chosen and the thermophysical properties are shown in Table 6.7. 
Table 6.7. Parameters of physical properties used in this model

\begin{tabular}{ccc}
\hline Parameters & Units & Values \\
\hline Temperature, $T$ & ${ }^{\circ} \mathrm{C}$ & 50 \\
Pressure, $P$ & $\mathrm{MPa}$ & 20 \\
Gravitational acceleration, $g$ & $\mathrm{~m} / \mathrm{s}^{2}$ & 9.81 \\
Density of brine, $\rho_{\text {matrix }}$ & $\mathrm{kg} / \mathrm{m}^{3}$ & 1051 \\
Density of $\mathrm{CO}_{2}, \rho_{\mathrm{CO}_{2}}$ & $\mathrm{~kg} / \mathrm{m}^{3}$ & 784.29 \\
Interfacial tension, $\sigma$ & $\mathrm{N} \cdot \mathrm{m}$ & 0.04 \\
Brine viscosity, $\mu_{\text {matrix }}$ & $\mathrm{Pa} \cdot \mathrm{s}$ & 0.0007 \\
$\mathrm{CO}_{2}$ viscosity, $\mu_{\mathrm{CO}_{2}}$ & $\mathrm{~Pa} \cdot \mathrm{s}$ & 0.000069 \\
\hline
\end{tabular}


Table 6.8. Distribution of pore body, pore throat, porosity and permeability in the six selected reservoirs.

\section{GCS Reservoirs}

\section{Outcrop-Fluvial Outcrop-Estuarine}

\begin{tabular}{|c|c|c|c|c|c|}
\hline Parameter & Distribution Type & $\bar{x}$ & $\sigma$ & $\bar{x}$ & $\sigma$ \\
\hline Pore Body (m) & Lognormal & $12.95 \times 10^{-5}$ & $0.49 \times 10^{-5}$ & $7.57 \times 10^{-5}$ & $0.62 \times 10^{-5}$ \\
\hline Pore Throat (m) & Lognormal & $2.59 \times 10^{-5}$ & $0.10 \times 10^{-5}$ & $1.51 \times 10^{-5}$ & $0.12 \times 10^{-5}$ \\
\hline Porosity (\%) & Normal & 0.261 & 0.044 & 0.261 & 0.045 \\
\hline Permeability $\left(\mathrm{m}^{2}\right)$ & Lognormal & $3252 \times 10^{-12}$ & $2.91 \times 10^{-12}$ & $1.025 \times 10^{-12}$ & $0.005 \times 10^{-12}$ \\
\hline & & \multicolumn{2}{|c|}{ Outcrop-Deltaic } & \multicolumn{2}{|c|}{ Buck Draw-Estuarine } \\
\hline Parameter & Distribution Type & $\bar{x}$ & $\sigma$ & $\bar{x}$ & $\sigma$ \\
\hline Pore Body (m) & Lognormal & $3.18 \times 10^{-5}$ & $0.77 \times 10^{-5}$ & $1.30 \times 10^{-6}$ & $1.04 \times 10^{-6}$ \\
\hline Pore Throat (m) & Lognormal & $0.63 \times 10^{-5}$ & $0.15 \times 10^{-5}$ & $2.60 \times 10^{-7}$ & $2.09 \times 10^{-7}$ \\
\hline Porosity (\%) & Normal & 0.24 & 0.038 & 0.054 & 0.03 \\
\hline \multirow[t]{2}{*}{ Permeability $\left(\mathrm{m}^{2}\right)$} & Lognormal & $15.9 \times 10^{-15}$ & $7.66 \times 10^{-15}$ & $1.68 \times 10^{-16}$ & $1.46 \times 10^{-16}$ \\
\hline & & \multicolumn{2}{|c|}{ Buck Draw-Fluvial } & \multicolumn{2}{|c|}{ Buck Draw-Deltaic } \\
\hline Parameter & Distribution Type & $\bar{x}$ & $\sigma$ & $\bar{x}$ & $\sigma$ \\
\hline Pore Body (m) & Lognormal & $3.59 \times 10^{-6}$ & $1.10 \times 10^{-6}$ & $6.63 \times 10^{-7}$ & $2.42 \times 10^{-7}$ \\
\hline Pore Throat (m) & Lognormal & $7.18 \times 10^{-7}$ & $2.2 \times 10^{-7}$ & $1.33 \times 10^{-7}$ & $0.48 \times 10^{-7}$ \\
\hline Porosity $(\%)$ & Normal & 0.085 & 0.027 & 0.062 & 0.024 \\
\hline Permeability $\left(\mathrm{m}^{2}\right)$ & Lognormal & $1.68 \times 10^{-16}$ & $1.64 \times 10^{-16}$ & $0.40 \times 10^{-16}$ & $0.07 \times 10^{-16}$ \\
\hline
\end{tabular}


The critical ganglion sizes were calculated for all the six reservoirs, as shown in Table 6.9. The critical ganglion sizes range from 0.88 to $172.46 \mathrm{~m}$ for different reservoirs. The reservoir with the greatest permeability, i.e. Outcrop Fluvial has the smallest critical ganglion size, vice versa for Buck Draw Deltaic. It should be noted that the ganglion size calculated with Eq. 6.2 is the apparent parcel size for $\mathrm{CO}_{2}$ when the plume initially starts to migrate. Once the movement ensues, ganglion is dispersed into smaller ones because of the instable front and the viscous fingering, i.e. "regenerated ganglions" [Wang and Bryan et al., 2013].

Table 6.9. Critical ganglion size and mean velocity calculated in the six selected reservoirs.

\begin{tabular}{c|cccccc}
\hline \multirow{2}{*}{ Reservoir } & Outcrop & Outcrop & Outcrop & Buck Draw & Buck Draw & Buck Draw \\
& Fluvial & Estuarine & Deltaic & Estuarine & Fluvial & Deltaic \\
& & & & & & \\
\hline Critical Ganglion Size $(\mathrm{m})$ & 0.88 & 1.51 & 3.59 & 17.85 & 31.85 & 172.46 \\
\hline
\end{tabular}

It should be noted that the critical ganglion size is a nominal scale considering $\mathrm{CO}_{2}$ plume as a continuum and does not mean the regenerated pore-scale $\mathrm{CO}_{2}$ parcels. Using the largest critical ganglion size among these six cases, i.e., 172.46m under the condition of Buck Draw Deltaic reservoir, Monte-Carlo stochastic analysis was conducted to evaluate the distributions of $\mathrm{CO}_{2}$ rise velocity using the Oracle ${ }^{\circledR}$ Crystal ball software. The results are shown in Figure 6.3. The distributions follow the pattern of normal distribution and the average velocity generally decreases with decreasing permeability. For the Buck Draw Estuarine and Fluvial cases, the distribution is more concentrated to the center because the standard deviation and the mean are on the same level of magnitude. Sensitivity analysis was carried out to evaluate the effect and relative importance of several parameters including pore body size, pore throat size, 
contact angle, viscosity, porosity and permeability. Results showed that the relative importance of different parameters was case-specific with permeability has the greatest effects, followed by contact angle and pore throat being the second or third.
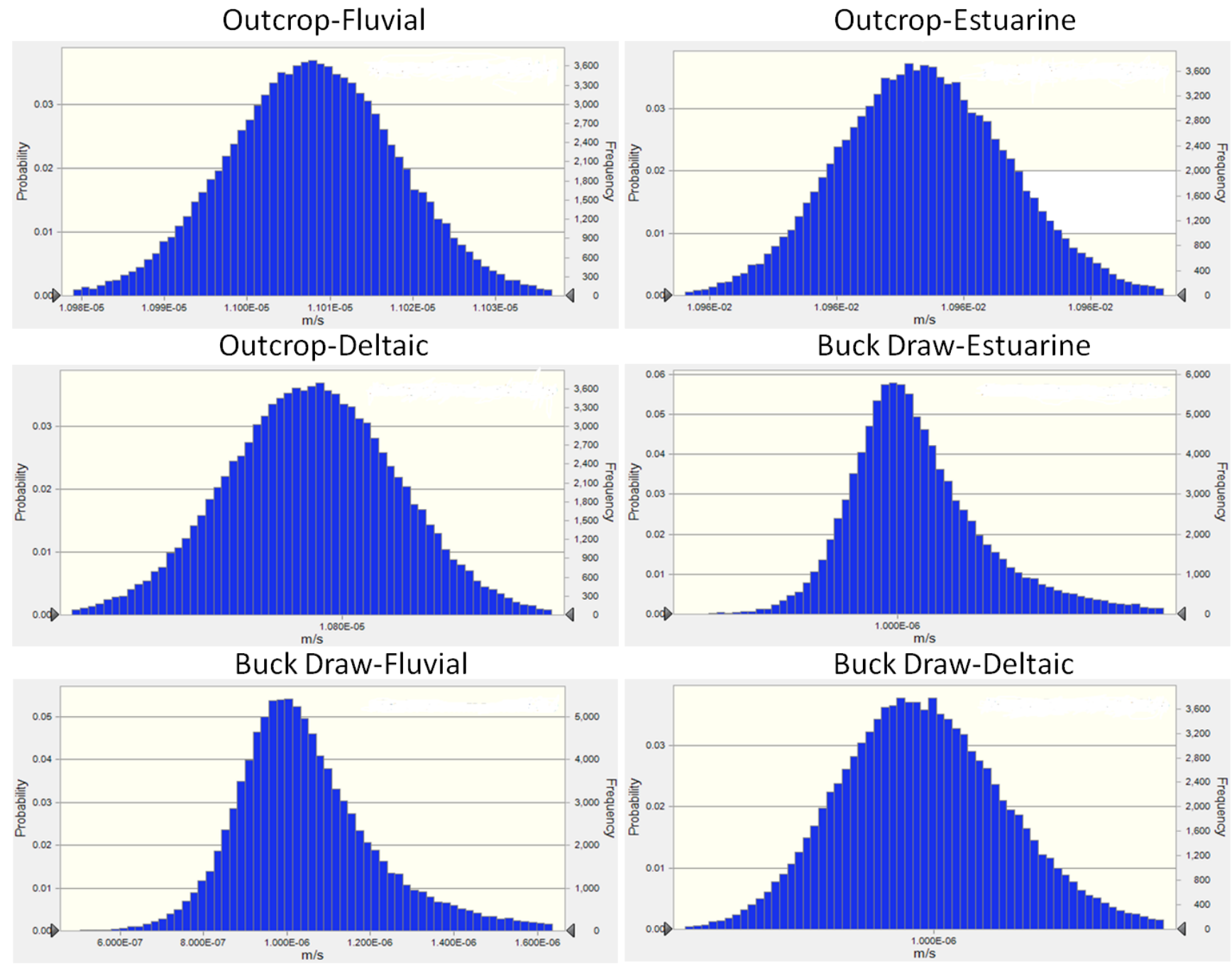

Figure 6.3. Distribution of rise velocity of $\mathrm{CO}_{2}$ ganglion through porous media in six selected reservoir in South Dakota: Outcrop fluvia, Outcrop estuarine, Outcrop deltaic, Buck Draw estuarine, Buck Draw fluvial and Buck Draw deltaic. Note that the scales of the $\mathrm{X}$ axis are not uniform between these subset plots: Outcrop Fluvial: $\sim 1.1 \times 10^{-5} \mathrm{~m} / \mathrm{s}$; Outcrop Estuarine: $\sim 1.1 \times 10^{-2} \mathrm{~m} / \mathrm{s}$; Outcrop Deltaic: $\sim 1.08 \times 10^{-5} \mathrm{~m} / \mathrm{s}$; Buck Draw Estuarine: $0.6 \sim 1.6 \times 10^{-6} \mathrm{~m} / \mathrm{s}$; Buck Draw Fluvial: $\sim 1.0 \times 10^{-6} \mathrm{~m} / \mathrm{s}$; Buck Draw Deltaic: $\sim 1.0 \times 10^{-6} \mathrm{~m} / \mathrm{s}$. 


\subsection{Discussion and conclusion}

Before geologic carbon sequestration can be widely deployed, it is imperative that leakage processes be better characterized. This work combined experimental results with applicable modeling frameworks to provide a first look at how parcels of $\mathrm{CO}_{2}$ would travel over long distances. The models are based on Stokes law and Ergun's equation for buoyancy driven flow of gas through porous media. Experimental data including detailed viscosity measurements and contact angle measurement allowed for more detailed analysis of the interfacial properties of this problem than had been reported in the past. Quantitative sensitivity analysis were performed to understand which parameters have the greatest impact on the bubble rise velocity and the ranking was found to change depending on the characteristics of the host formation.

Despite the advances reported here, this work only begins to describe the behavior of $\mathrm{CO}_{2}$ vertical transport in the subsurface. There are several simplifying assumptions made here that should be explored in further detail including the assumption that the fluids in this problem are incompressible. Gas density varies with pressure and the nonzero flux on the surface results in a "slip flow". There is also the possibility that hysteretic pathways will form through which surface chemistries are altered, e.g., low-pH brine would react with aluminosilicate or carbonates to expose different formations underneath. Mass transfer between $\mathrm{CO}_{2}$ bubbles and surrounding brine could further complicate the process. Surface roughness, heterogeneity, fractures and adhesion effects need to be accounted. Finally, instability such as viscous figuring created in the heterogeneous porous media will certainly impact the assumptions made here about vertical flow. By combining experimental and modeling techniques, each of these factors can be described so that leakage from GCS sites can be anticipated and prevented. 


\section{Chapter 7}

\section{Conclusions and Future Work}

Geologic carbon sequestration may be the most attractive option for mitigating the emissions that are leading to anthropogenic global warming. However, the risk that injected $\mathrm{CO}_{2}$ could leak from target formations is the single biggest technological obstacle associated with deployment of the technology. The underlying physicochemical processes that would control leakage processes are poorly understood. Research is needed to better understand this risk such that it can be avoided and the most appropriate repository sites can be selected. At the most fundamental level, an improved understanding of $\mathrm{CO}_{2}$ and brine multi-phase flow through porous media under relevant geophysical and geochemical condition must be developed. The interfacial phenomena between gaseous, liquid or supercritical $\mathrm{CO}_{2}$, connate brine and reservoir solids are key to resolving the relevant transport processes.

Several interfacial processes will impact the rise of a $\mathrm{CO}_{2}$ parcel through porous media. Three main forces act on a rising $\mathrm{CO}_{2}$ parcel, i.e., buoyancy force resulted from density difference between $\mathrm{CO}_{2}$ and brine, interfacial tension force originated from the interactions between $\mathrm{CO}_{2}$ and its brine and solid environment, and the shear drag force caused by the friction between 
$\mathrm{CO}_{2}$ and the viscous brine. Buoyancy force is determined by the earth's gravity. Interfacial tension force is affected by interfacial tension and wettability, both of which required more extensive experimental characterization. And the shear drag force is strongly affected by the viscosity of the surrounding brine and the shear applied on the $\mathrm{CO}_{2}$ mass. A lack of experimental data for viscosity and contact angle makes it difficult to evaluate the overall viability of $\mathrm{CO}_{2}$ sequestration at the reservoir scale. This thesis sought to fill the gap in the knowledge that existed related to these parameters.

The objective of this work is to develop a fundamental understanding of how interfacial properties at the $\mathrm{CO}_{2}$-brine-mineral interfaces could influence buoyancy driven flow through porous media. This work begins to address the need to study the chemical and physical processes governing vertical flow of $\mathrm{CO}_{2}$ in a controlled laboratory setting spanning multiple length scales. The results presented herein include a combination of improved experimental results including viscosity, contact angle and interfacial adhesion. Relevant modeling frameworks are developed to shed light on how parcels of $\mathrm{CO}_{2}$ would travel over long distances.

\subsection{Summary and Conclusions}

To fill the gap in understanding whether multiphase $\mathrm{CO}_{2}$-brine rheology will enhance or suppress leakage, this work explored the viscosity of single and multiphase mixtures of saline water in contact with $\mathrm{CO}_{2}$ over a range of GCS in-situ conditions, especially for pressures at or below $10 \mathrm{MPa}$. These results, presented in Chapter 3, suggest that during leakage events, once vapor phase $\mathrm{CO}_{2}$ is present, discrete $\mathrm{CO}_{2}$ bubbles exhibit complex linear viscoelastic, time de- 
pendent and thixotropic behavior. The apparent viscosity of these mixtures could be significantly higher than brine alone but the microstructure was easily destroyed, and not readily regained, under high shear conditions. Higher temperatures and higher ionic strength make the effect less pronounced. The results demonstrate that the ebullition and evolution of $\mathrm{CO}_{2(\mathrm{~g})}$ bubbles could act to impede vertical flow of $\mathrm{CO}_{2}$-brine mixtures through porous formations in as much as these bubbles will alter the effective viscosity of the mixture.

In Chapter 4, the results of extensive wettability tests were reported. These experiments sought to understand the wetting behavior at the $\mathrm{CO}_{2}$-brine-mineral interface over a broad range of pressure, temperature, $\mathrm{pH}$ and ionic strength conditions for a variety of mineral surfaces. The results suggest that solution chemistry has an important impact on controlling contact angle. Specifically, its role in determining the $\mathrm{pH}$ at the point of zero charge $\left(\mathrm{pH}_{\mathrm{pzc}}\right)$ of the surface was revealed. The mass transfer and phase change effects on contact angle were also observed. Under non-equilibrium conditions such as mass transfer, hysteretic effects were observed when $\mathrm{CO}_{2}$ was dissolving into the bulk fluid and this effect varied between minerals. Contact angle was found to increase during the $\mathrm{CO}_{2}$ phase transition from liquid to gas. Sensitivity analysis suggests that the $38 \%$ differences in contact angle reported here could manifest $5-10 \%$ differences in capillary trapping or pressure.

Chapter 5 explores the role of adhesion at the mineral brine interface when contacted with a non-wetting gas. These experiments were carried out using the same contact angle testbed used in Chapter 4 but here the effects on surface energy were much more pronounced and found to be influenced by the chemistry of the mineral surface, the chemistry of the brine solution, and the roughness of the surface. The strength of adhesion was surprisingly strong and neither fierce turbulence nor tilting could remove the attached droplets. Contact angle hysteresis and the 
strength of adhesion were tested on several typical minerals. The effect was most pronounced on phlogopite in contact with connate brines where over $50 \%$ of bubbles adhered to the surface. In $\mathrm{CO}_{2}$, adhesion was stronger and more reproducible on phlogopite compared to silica. Adhesion happened on silica in one out of the three replicates. Measurements on illite, kaolinite, quartz and calcite showed little to no adhesion. A $\mathrm{N}_{2}$ control also showed adhesion on phlogopite and silica under the same conditions in the $\mathrm{CO}_{2}$ experiments. This time, the reproducibility and strength of the adhesion trend was reversed that $\mathrm{N}_{2}$ showed stronger and more frequent adhesion on silica than phlogopite. $\mathrm{CO}_{2}$ bubbles adhered to the surface typically showed distorted ternary phase lines suggesting that some degree of contact line pinning was taking place. $\mathrm{pH}$ played a determining role that adhesion was not observed where strong acid or base had been added to the synthetic brine used here. The incidence of adhesion increased with ionic strength in the solution and the carbonation of the brine.

In Chapter 6, an improved force balance with better estimates of the shear force and interfacial tension force terms for predicting vertical migration of $\mathrm{CO}_{2}$ from geologic sequestration sites was established using the experimental results of viscosity and contact angle collected in Chapters 3-5. The results were compared to the recently published ganglion model derived by Wang, Bryan et al. [2013]. The results suggest that wettability, porosity, pore throat, grain size and bubble size are all important for controlling leakage and that the priority depends on the characteristics of the formation. Using the ganglion model, velocity distributions were calculated using published data from seven $\mathrm{CO}_{2}$ storage sites. The critical ganglion sizes that would initiate buoyancy-driven flow of $\mathrm{CO}_{2}$ were calculated respectively for all the seven cases. At smaller scales, this enhanced model could help explain preferential flow pathways and potential hystere- 
sis that could influence leakage from GCS sites. At larger scales, the results of this work could contribute to more accurate prediction tools for managing the risk associated with GCS.

\subsection{Recommendation for Future Research}

Further research in several key aspects is necessary to deploy GCS on a broader scale soon. These aspects include: (1) identifying mineral species that are $\mathrm{CO}_{2}$-adhesive and testing these; (2) better understanding the mechanism of adhesion by conducting molecular dynamics simulations; (3) further improving the existing modeling framework with the experimental data of viscosity, interfacial tension, contact angle and the adhesion effects accounted; (4) validating the improved model with small and intermediate scale column studies.

\subsubsection{Identify $\mathrm{CO}_{2}$-Adhesive Minerals}

Adhesion has been demonstrated in this work to have great capacity to immobilize injected $\mathrm{CO}_{2}$ and facilitate other trapping mechanisms. Since adhesion could play a very important role in mitigating leakage, its trapping advantages should be fully characterized so that they can be used to maximize trapping. To date, only a few minerals including phlogopite, silica and calcite have been found to adhere $\mathrm{CO}_{2}$. And the occurrence of these minerals on the pore surface in GCS reservoirs is generally not high. Other mineral species exist which have similar interfacial properties as the above-mentioned adhesive minerals. And this similarity should derive alike interfacial behaviors and we expect to observe adhesion of $\mathrm{CO}_{2}$ on such minerals. In the search for the underlying mechanism driving the adhesion process, and other surfaces that might exhibit the same properties, two directions will be pursued. The first would be to look for minerals which 
have atomically smooth surfaces because adhesion is a manifestation of nano-meter scale short range van der Waals interactions and the smoother the surface is, the easier it is for $\mathrm{CO}_{2}$ to get really close to the solid surface and thus initiate adhesion. The second is to look up minerals whose molecular structures resemble those of known adhesive minerals. For example, the trioctahedral mica family consists of biotite, lepidolite, muscovite, phlogopite, zinnwaldite, etc., which share very similar level of smoothness, molecular formula, molecular structure, molecular-level affinity with $\mathrm{CO}_{2}$. Preliminary screening should start with the very smooth trioctahedral mica members.

\subsubsection{Explore the Mechanism of Adhesion with Molecular Dynamics Simulation}

Although the adhesion phenomena have been well characterized, the experimental data we have collected and the phenomena we have discovered so far are not adequate to fully understand the occurrence and the function of $\mathrm{pH}$, ionic strength and the carbonation. More work is needed on the underlying mechanism of adhesion so that favorable conditions can be created to promote it. Molecular dynamics simulation is a powerful tool to learn the interaction mechanisms at the molecular level and would be a useful means to reveal the functions that dominate adhesion. With a well-structured simulation near the interface, the complex van der Waals interactions between the solid, brine solution and $\mathrm{CO}_{2}$ including the modified charge distribution at the solid surfaces, electric double layer and hydration layer structures by $\mathrm{pH}$ and ionic strength, the solvation of ions, the reorientation of water and $\mathrm{CO}_{2}$ molecules, the surface complexation by carbonate species, cation exchange, dipole-dipole interaction, dipole-induced dipole interaction, etc., could be clarified and the relative importance of these interactions could be evaluated. 


\subsubsection{Improve Modeling Framework of $\mathrm{CO}_{2}$ Transport}

As stated in Chapter 2 and 6, the ultimate goal of this work is to contribute to robust modeling frameworks that are capable of accurately simulating $\mathrm{CO}_{2}$-brine multiphase flow through porous media by comprehensively handling the complex geochemical, geophysical, geomechanical and geothermal behaviors at the reservoir scale. Before integrating all the factors into one program, additional work is needed to further improve existing modeling frameworks. The two modeling frameworks used in this work, i.e. the Corapcioglu method and the ganglion model can be the starting point. Sensitivity analysis is also very important to evaluate the relative importance of all the impacting factors so that efforts and resources can be wisely allocated to investigate the parameters that matter the most in the $\mathrm{CO}_{2}$ transport processes. The existing models could be enhanced by identifying opportunities to include mass transfer, geochemical reactions, heat transfer, wettability hysteresis, adhesion, permeability hysteresis, compressibility of flow and solids, fingering and flow instability. Upscaling is another tough challenge where the compatibility of parameters and computational methods could cause extra issues. Sophisticated modeling frameworks such as TOUGH2, a multi-phase fluid and heat transfer model for porous media will be a good option to test formulated hypotheses and validate experimental results [Pruess, 2004].

\subsubsection{Model Validation Using Small/Intermediate-Scale Columns}

Design, construction and deployment of small-scale and intermediate-scale pressurized columns in which vertical flow of $\mathrm{CO}_{2}$ are necessary to validate many of the parameters and modeling tools developed here. This work will be the first step in upscaling the results presented here by enabling controlled experiments under a variety of GCS-relevant scenarios that mimic 
deep sedimentary formations with respect to depths (pressures) and thermal gradients with careful pressure and temperature control. A two-level full factorial experimental design will be employed to fully characterize the influence of the four process parameters, namely pressure, temperature, sediment type and rate of $\mathrm{CO}_{2}$ addition, on $\mathrm{CO}_{2}$ breakthrough and distribution in the column. A one-way analysis of variance will be performed on the results from the cooling studies to determine which parameters were significant. With this design, $\mathrm{CO}_{2}$ breakthrough times under different conditions, preferential leakage pathways, capillary trapping of $\mathrm{CO}_{2}$ and effect of packing surface properties on leakage rates are the first few questions to be explored. The interdependence of buoyancy-driven flow, Joule-Thomson cooling, phase change, thermal exchange, viscous fingering, residual gas trapping, $\mathrm{CO}_{2}$ dissolution and its effect on native fluids, which is one of the least understood processes in the context of GCS should be studied. The length scales should enable reproduction of the capillary-level processes governing multi-phase flow in porous media in the context of the meter-scale variation in pressure and temperature governing $\mathrm{CO}_{2}$ phase properties. An example design of these columns is shown in Figure 7.1. The column study will be a useful tool for eventually bridging the gap between bench-scale measurements, molecular-level and large-scale simulations, and field-scale demonstration. 

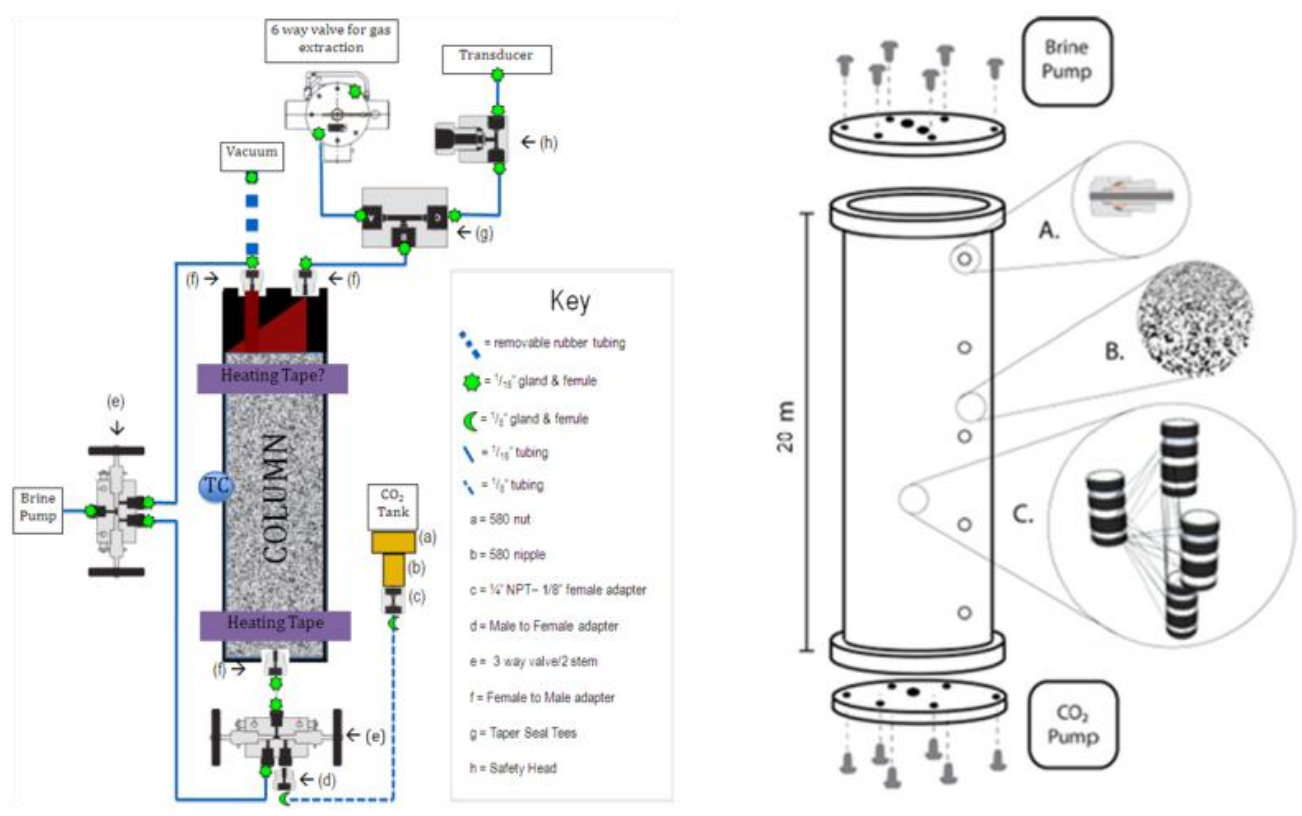

Figure 7.1. Schematic of column design. (Left). Small-scale $(\sim 1 \mathrm{~m})$ column; (Right). Intermediate-scale $(\sim 10 \mathrm{~m})$ column A. custom-built stainless steel pressure vessel with flange closures and possibility for coaxial configuration if needed for sampling; B. packed media with properties that can be specified a priori and changed between experiments; C. Ports for thermocouples and pressure transducers; D. In-situ resistivity arrays will allow for real-time imaging of the $\mathrm{CO}_{2}$ front as it migrates up the column. 


\section{Appendices}

\section{Appendix A: Supplemental Methods}

\section{A.1. Delivery of High-Pressure $\mathrm{CO}_{2}, \mathrm{~N}_{2}$ and Brine}

High purity, high-pressure gaseous, liquid and supercritical $\mathrm{CO}_{2}$ was used throughout this research project. Supercritical $\mathrm{N}_{2}$ was also used in the wettability experiments as blank control. Food-grade high purity $\mathrm{CO}_{2}$ and $\mathrm{N}_{2}(>99 \%$, from Roberts Oxygen Co., Charlottesville, VA) were both used as received and directly filled into a Teledyne $\mathrm{ISCO}^{\circledR} 500 \mathrm{HP}$ high-pressure syringe pump (flow rate: $0.001-204 \mathrm{ml} / \mathrm{min}$; pressure range: 0.07-34.47 MPa) with a constant temperature jacket. Then $\mathrm{CO}_{2}$ and $\mathrm{N}_{2}$ were pressurized to the specified experimental pressure conditions. The synthetic brine solutions at a broad range of $\mathrm{pH}$ and ionic strength which mimics the connate brines were transported using a LabAlliance ${ }^{\circledR}$ Series IV HPLC pump (flow rate: 0.01-9.99 $\mathrm{mL} / \mathrm{min}$; pressure range: $0-41.38 \mathrm{MPa}$ ). The syringe pump and the HPLC pump are shown in Figure A.1. 

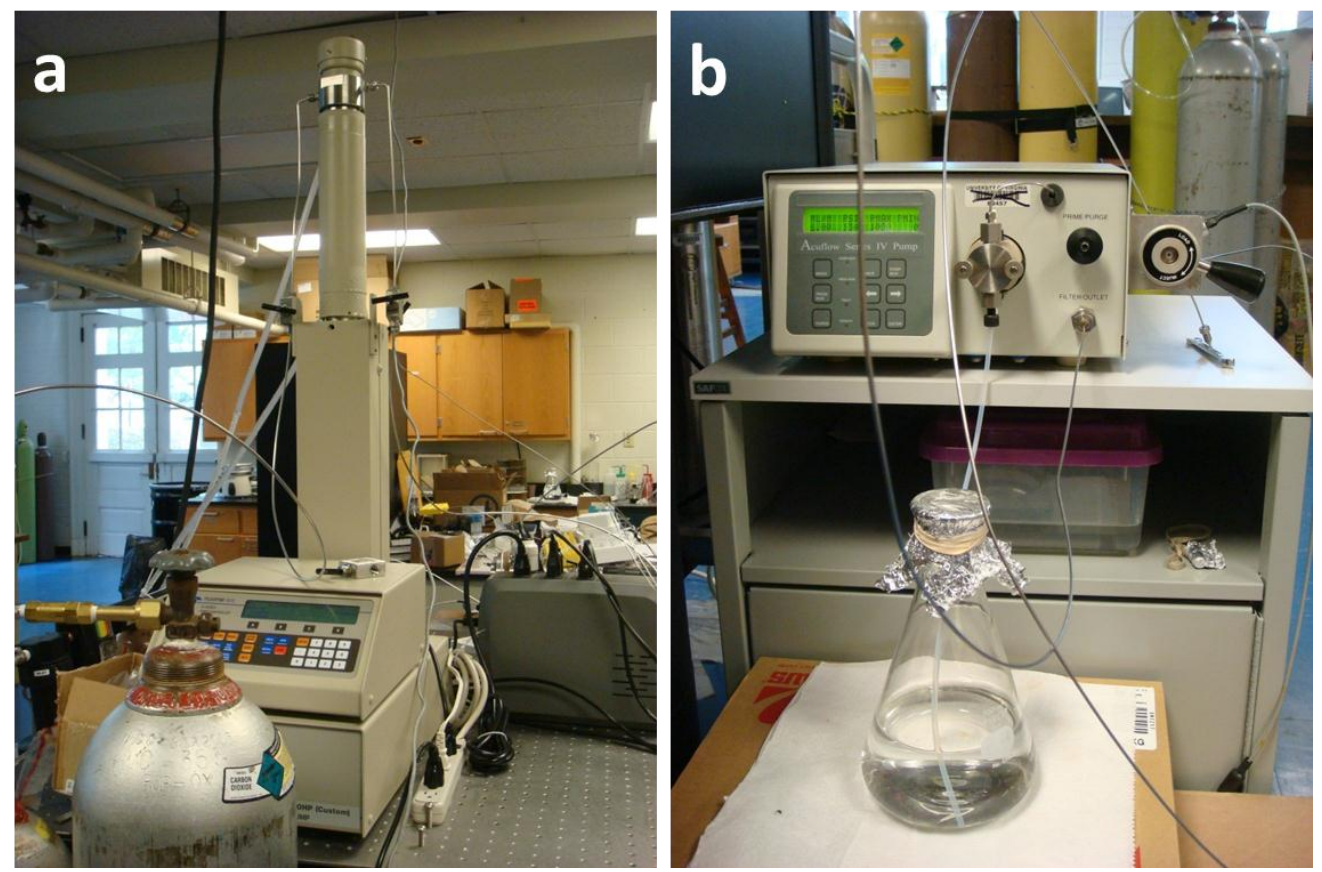

Figure A.1. High pressure pumps used in this work. a. Teledyne ISCO $^{\circledR} 500 \mathrm{HP}$ high-pressure syringe pump (left); b. LabAlliance ${ }^{\circledR}$ Series IV HPLC pump (right).

The high pressure test beds in this research were connected via 1/16 inch high pressure 316 stainless steel tubing (pressure range: 0-103.45 MPa). In-flows and out-flows were controlled using taper-seal two-way or three-way valves (pressure range: 0-103.45 MPa). One-way valves were used between $\mathrm{CO}_{2}$ canister and the syringe pump to prevent backflow. Safety pressure relief valves with rupture discs (pressure range: 0-34.47 $\mathrm{MPa}$ ) were connected to the test beds to prevent over-pressure. The majority of fittings used in this work were taper-sealed with ferrules and glands to ensure leak-free sealing. NPT fittings were only used for pressure transducers and gas canisters. Male to male and female to male adapters were used when needed. All these mentioned connection, fitting and coupling parts were supplied by High Pressure Equipment Company (Erie, Pennsylvania) and were shown in Figure A.2. 


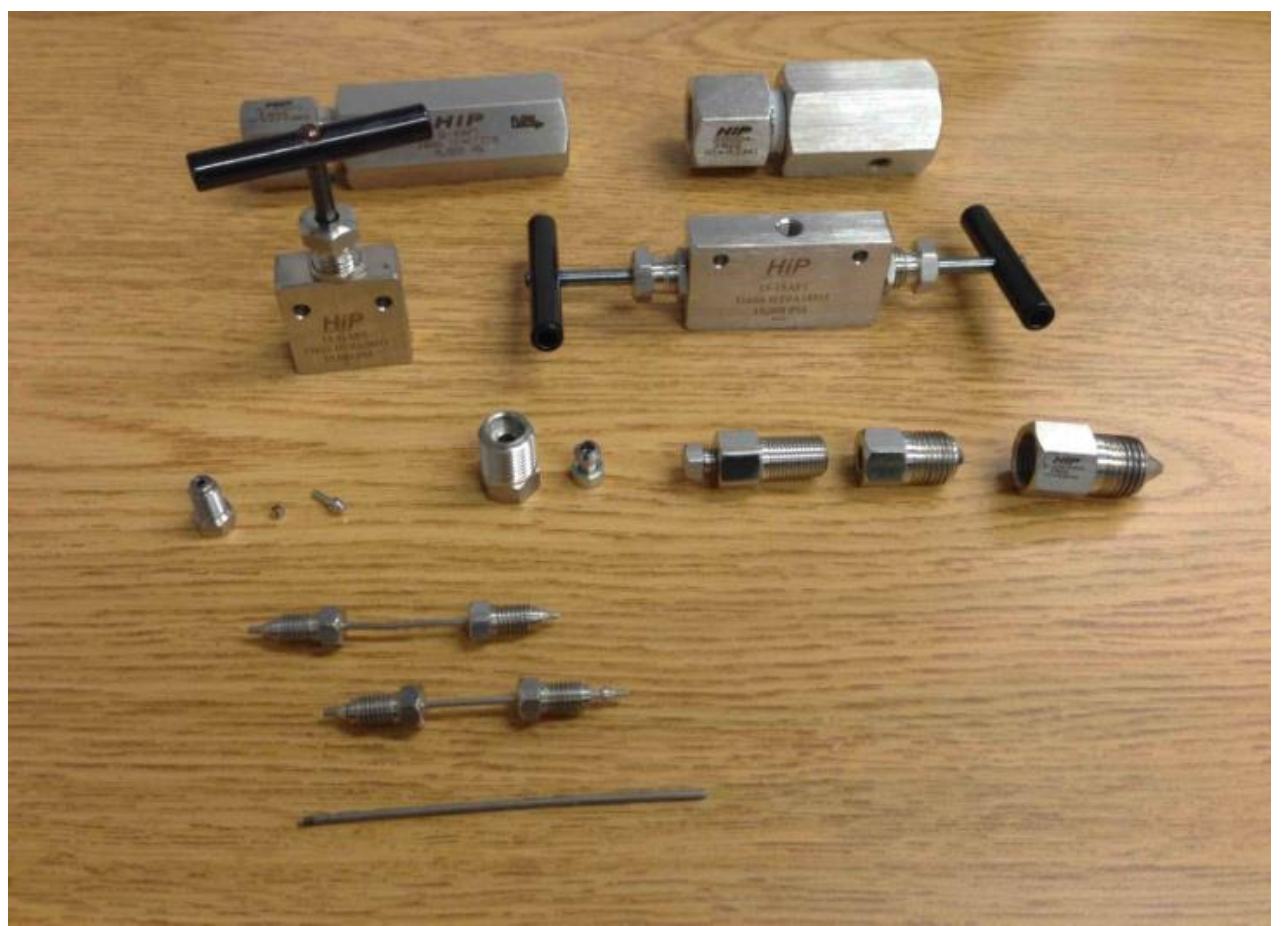

Figure A.2. Connection, fitting and coupling parts used in this work including taper-seal twoway and three-way pressure valves, one-way valve, safety pressure relief valve, 1/16 inch high pressure 316 stainless steel tubing, taper-seal ferrules and glands, and male to male and female to male adapters.

\section{A.2. Design of High-Pressure Vessel}

The manuscript of the original design for the high-pressure vessel used in wettability research is shown in Figure A.3. This cell was also used as view cell in the rheology work to observe the phase states of $\mathrm{CO}_{2}$-brine mixtures. 


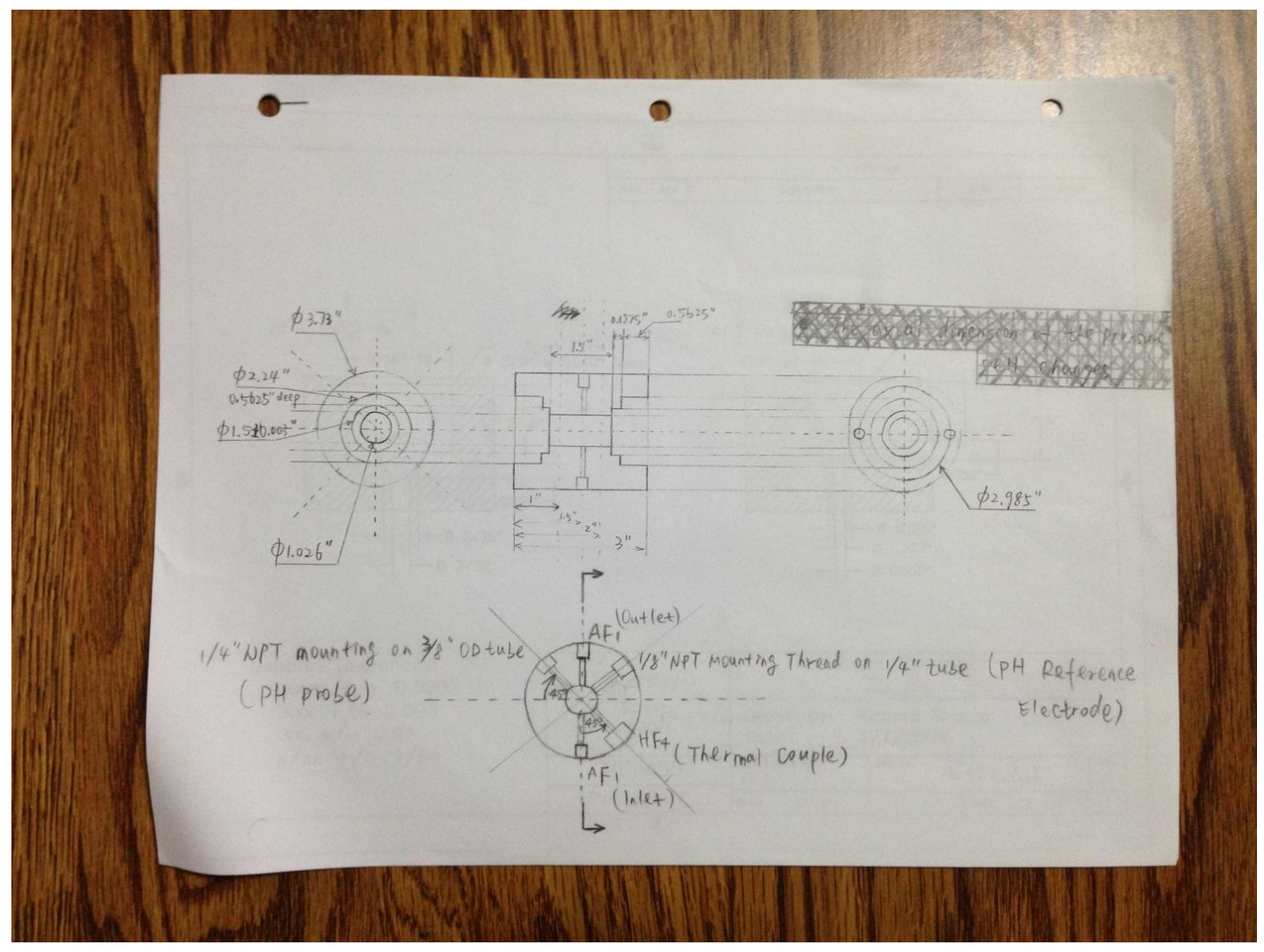

Figure A.3. Draft of design for the high-pressure vessel.

\section{A.3. Sample Preparation}

The mineral specimens used in the wettability and adhesion studies were prepared using the methods described in Chapter 4. An example specimen in the sample holder before being placed into the pressure vessel is shown in Figure A.4. 


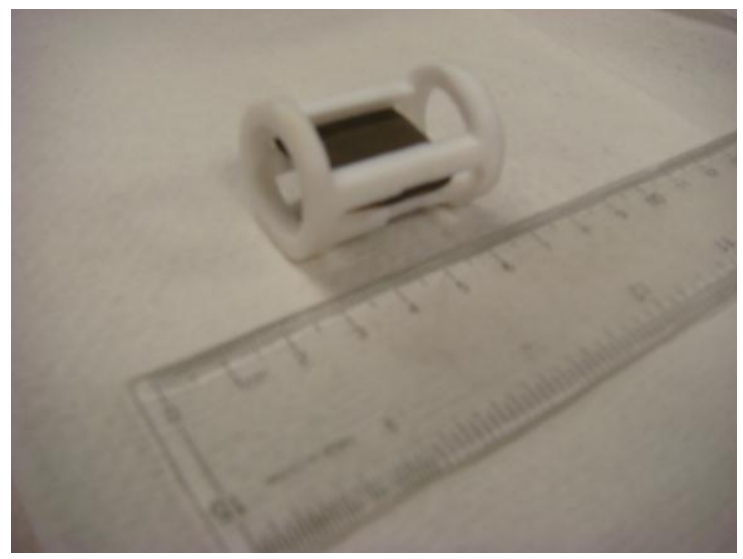

Figure A.4. An example mineral specimen in sample holder before being placed into the pressure vessel.

\section{A.4. Drop Image Analysis}

The image outputs from the CCD camera were distorted in terms of the aspect ratio of the image. A correction factor was provided by the manufacturer and this was used to adjust the images to obtain realistic records of each bubble. Photo-editing software (Paint.NET) was used to resize, invert and crop the images in a manner compatible with the image analysis code ImageJ. Two contact angle algorithm plugins, i.e., DropSnake and LB-ADSA were used and DropSnake was found to give more accurate results but both were used throughout the work to cross reference measurements and ensure that angles were as accurate as possible. All the information of the diameter, volume, length of contact line, surface area and contact area of $\mathrm{CO}_{2}$ bubbles were acquired from LB-ADSA with input of the pixel density directly measured on the adjusted images. The steps associated with processing the images and analyzing the data are described in Figure A.5 with example images. 

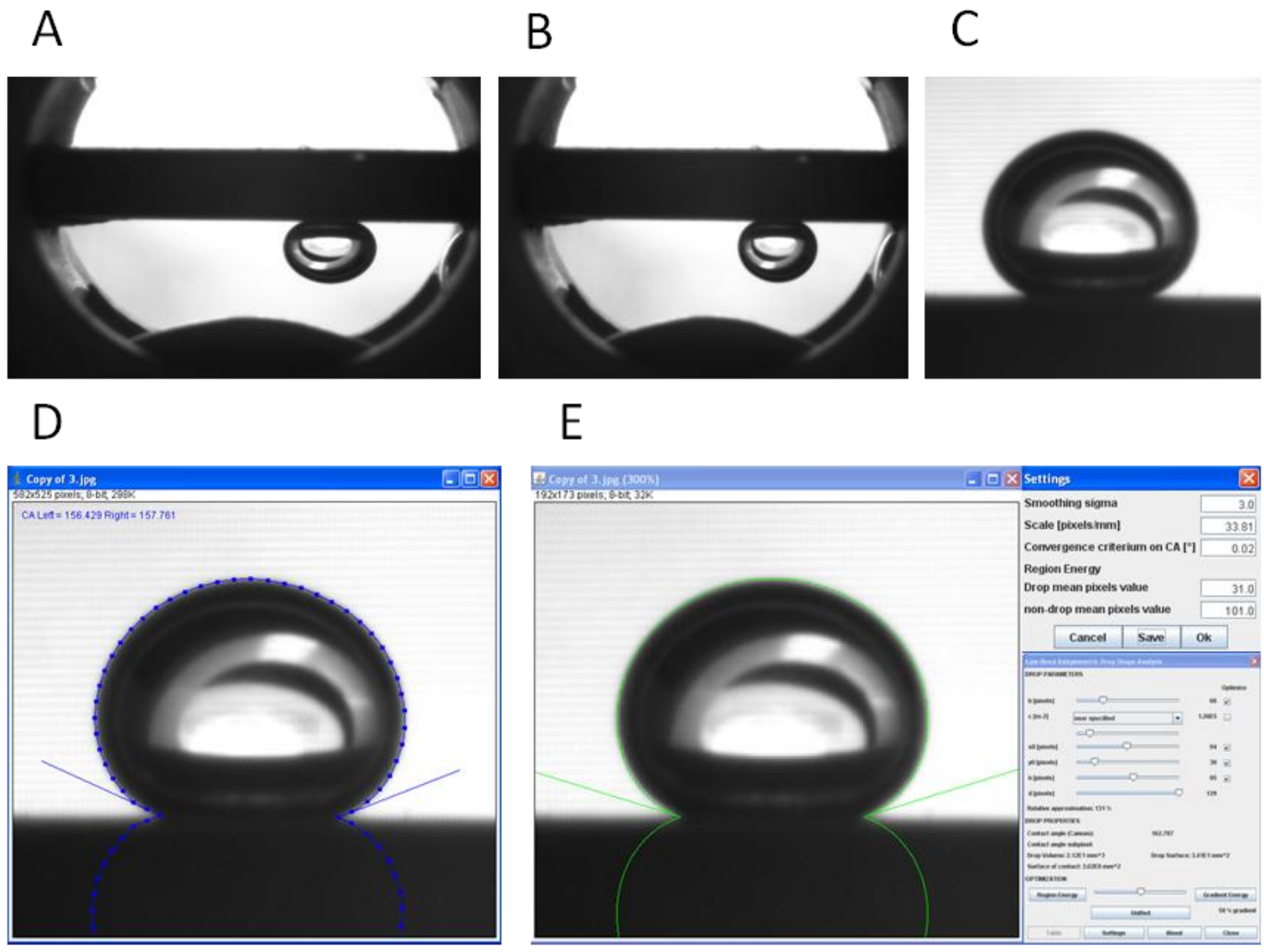

Figure A.5. Image analysis process: A - original images acquired from digital camera, B - adjusted images with correct aspect ratio, $\mathrm{C}$ - inverted and cropped images for contact angle measurement, D - analyzed image with "Dropsnake" line and contact angle values, E - analyzed image with "LB-ADSA" line and input (pixel density) and output (volume, surface area, contact area etc.).

\section{A.5. Two-Valve System to Precisely Control $\mathrm{CO}_{2}$ Droplets}

A "two-valve" control system was invented to precisely add/withdraw trivial amount of $\mathrm{CO}_{2}$ mass to/from the moving $\mathrm{CO}_{2}$ droplets during the wettability hysteresis (advancing and receding) test. The unique advantage of the "two-valve" setting is that very delicate pressure gradi- 
ent $(<10 \mathrm{psi})$ could be repeatedly created by coordinating the two valves. This method has been compared with the syringe pump which has a highly precise pressure control function. It was demonstrated that the "two-valve" setting offers better performance

\section{A.6. Precautions on Contact Angle Measurements}

Precautions were used to conduct measurements of contact angle and ensure the accuracy of measurement. Up to date, the majority of the methods employed to determine the magnitudes of contact angle are based on the principles of the Axisymmetric Drop Shape Analysis (ADSA) methodology. The prerequisite of the ADSA method is the symmetry of the droplet geometry whereas the droplets in many cases in reality have asymmetry caused by roughness, heterogeneity, contamination, adhesion and hysteresis, etc. Besides, because the values of contact angles are determined by drawing the tangential lines at the two ternary-phase contact points along the $\mathrm{CO}_{2}$-brine interface, even one pixel misplacement could result in an error of a few degrees. Therefore, the two ternary-phase contact points must be carefully chosen. Most of the software packages conducting automated contact angle measurements have the two mentioned limitations. The Dropsnake algorithm (embedded in ImageJ software) used in this work can be applied on droplets of any shape by means of placing the measuring line with a fine string of points along the contour of the droplets by the user and the line then being optimized by the algorithm. It resolves the symmetry and contact point misplacement limitations and thus is the most flexible and reliable method to determine contact angle with the best accuracy. When conducting contact angle measurement, the measuring line well followed the curvature of the droplet and possess good smoothness. All the tested images with the measuring line were carefully compared with the 
original image side by side to minimize error. The combined manual and automatic determination of contact angle require appreciably more time and efforts but guarantee results of best precision.

\section{A.7. Effects of Preparation Methods on Wettability: PVA Coating for Measurement of Powdered Clays}

Surface preparation methods cause differences in contact angle results of clay species. Here clay samples were prepared in two different ways and the effects on wettability were assessed. The effect on cleaved solid samples of clay was compared to powered samples that have been thoroughly cleaned then used to coat a homogeneous glass slide. The results help translate laboratory results, on highly idealized surfaces to field scale work on clay surfaces that have resulted from diagenesis.

The coated surface of kaolinite appears in Figure A.6.

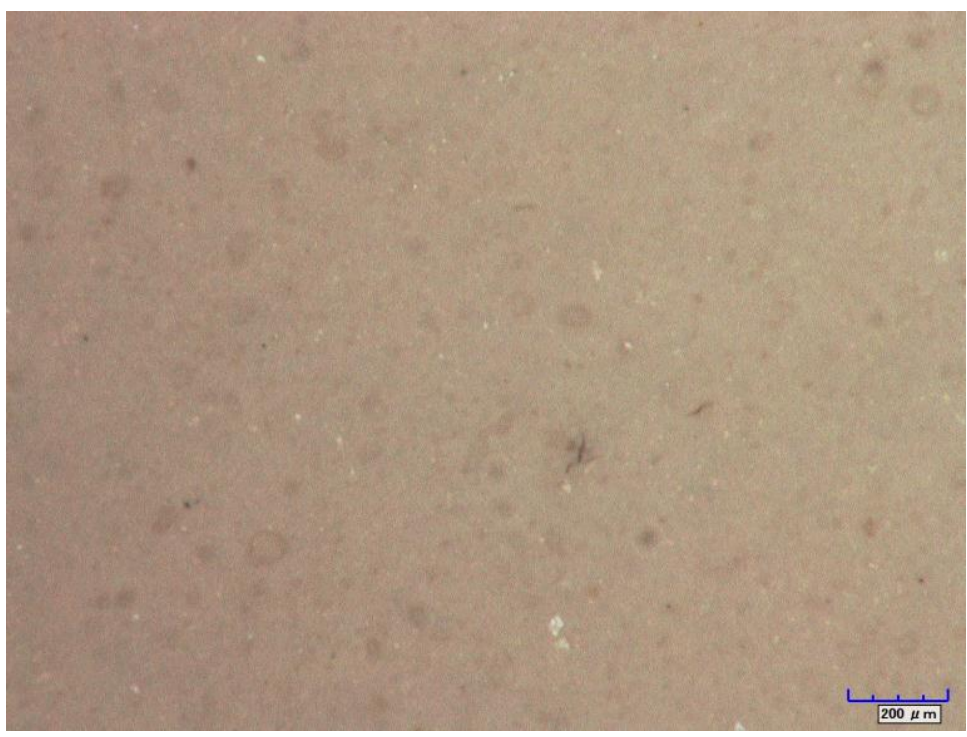

Figure A.6. Kaolinite powders were coated on high-purity glass slides using PVA 
The protocols for coating and dialysis are as below:

\section{Protocol: Dialysis}

- Pretreatment:

Step 1

Calculate the length of dialysis tubing.

(2 inches at end for handling)

Step 2

Soak in DI water for 30 mins.

Step 3

Fold one end and pinch with the clamp.

Step 4

Pipetting small amount of DI water to test leakage, rinse with DI water.

- Dialysis

Step 1

Fill the sample into the tubing using a plastic funnel.

Step 2

Remove the air bubbles.

Step 3

Place the tubing in a large beaker.

Step 4

Fill the beaker with DI water (the volume of DI water is roughly 10 20 times of the sample volume), add a stir bar and put the beaker on a stir plate. For low-molecular weight salts, equilibrate with stirring for 3 hours.

Step 5

Change the DI water if necessary.

Step 6

Monitor the conductivity of the DI water buffer. 


\section{Protocol: Coating Clays}

- Pretreatment of Clay:

Step 1

Remove organic matter with $\mathrm{H}_{2} \mathrm{O}_{2}$

Step 2

Citrate-Dithionite method to remove Fe oxides

Step 1

Measure 1 4 g dry and fine clay

Step 2

Add $2 \mathrm{~g}$ sodium dithionite and $20 \mathrm{~g}$ sodium citrate to

$120 \mathrm{ml}$ DI water

Step 3

Put clay into the beaker

Step 4

Shake for 16 hours at room temperature

Step 5

Add $120 \mathrm{ml}$ DI water and add 5 10 drops of 0.2\%

"Superfloc" solution

\section{Step 6}

Shake vigorously for 10 seconds, centrifuge.

\section{Step 7}

Damp the supernatant fluid

\section{Step 3}

Do size fractionation for clay by gravity sedimentation to $2 \mu \mathrm{m}$, repeat this for 2 3 times

Step 4

Wash the clay with $0.5 \mathrm{M} \mathrm{CaCl}_{2}, 1 \mathrm{M} \mathrm{NaCl}$ and $1 \mathrm{M} \mathrm{KCl}$ to obtain $\mathrm{Ca}$ -

smectite, Na-kaolinite, and K-illite 


\section{Step 5}

Dialyze the clay with DI water until electrical conductivity of the dialysate less than $5 \mu \mathrm{S} / \mathrm{cm}$

- Coat the microslides

\section{Step 1}

Prepare clay suspensions to be $40 \mathrm{~g} / \mathrm{L}$

\section{Step 2}

Mix the suspension with $80 \mathrm{mg} / \mathrm{L}$ PVA under $\mathrm{pH}=5$ and manually agitate for $30 \mathrm{mins}$

\section{Step 3}

Immerse the glass microscope slides in the suspension for an hour

\section{Step 4}

The mixture is dried at $80{ }^{\circ} \mathrm{C}$ for 24 hours 


\section{Appendix B: Supplemental Results}

\section{B.1. Dissolution/Precipitation at the Mineral Surface}

During the acid/base titration experiments discussed in the manuscript, significant dissolution of calcite at the $\mathrm{CO}_{2}$ contact was observed. Etching was visible on the surface of the sample and for long (several days) experiments a pit formed where the $\mathrm{CO}_{2}$ bubble was located as shown in Figure B.1. The dissolution of calcite in an acidic brine was expected, but the layered nature of dissolution, whereby fresh calcite is continually exposed supports the hypothesis that little hysteresis should be observed on the pure samples.

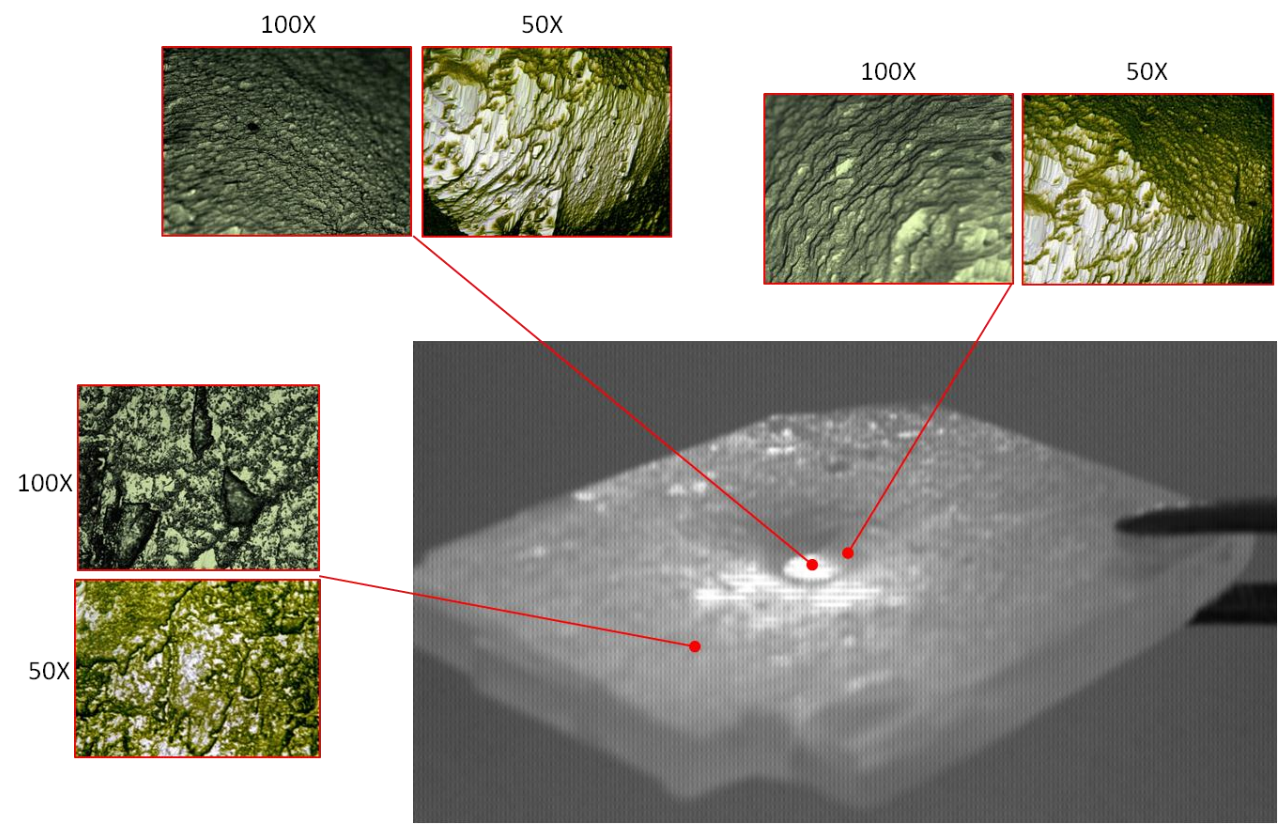

Figure B.1. Dissolution and surface morphology of calcite during the $\mathrm{pH}$ effect experiments suggest how hysteresis may impact contact angles. Images collected using an optical microscope at $50 \times$ and $100 \times$ magnification. 


\section{B.2. Hysteresis with Reaction}

For all of the minerals tested here, dissolution revealed more of the same mineral beneath the surface. We weighted the mass loss of calcite sample over the 10-day experiment and dissolution rate was found to be comparable with published values. In practice, dissolution would likely reveal a different mineral beneath the pore surface and the newly exposed mineral could have a different contact angle. Because of the homogenous nature of our samples the fact that no hysteresis was observed (Figure A.8) here does not mean that wettability driven pathways would not open up in host formations. It simply suggests that experiments like this will need to be coupled with reactive transport models to understand how preferential flow pathways could evolve.

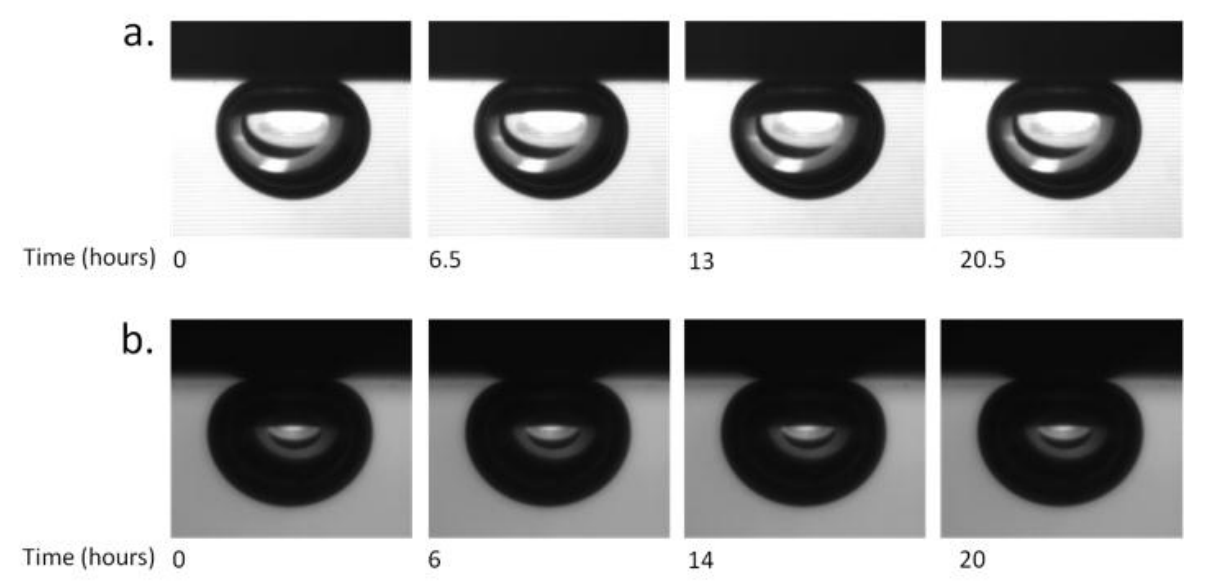

Figure B.2. No hysteresis was observed for a. calcite (Condition b in brine) and b. microcline (Condition a in brine) at equilibrium. 


\section{B.3. Control Minerals}

The mineral samples used here were from naturally occurring samples so separate rock samples were obtained from independent sources to validate the measurements collected here. All experiments were carried out on a second calcite sample, Iceland spar, obtained from Ward's Natural Science. A t-test was run on the results to understand whether there are statistically meaningful differences between the 8-9 replicates we had for each condition. The results, presented in Table A.1, show no statistically significant difference between the Iceland spar and the calcite over all the conditions tested here. P-values less than 0.05 indicate correlation.

Table B.1. T-test results for calcite and Iceland spar from different sources showed that there was no statistically significant difference between contact angle measurements in our system.

\begin{tabular}{ccc}
\hline $\begin{array}{c}\text { Solution Chemis- } \\
\text { try }\end{array}$ & P-T Conditions & P-value \\
\hline Buffered brine & $7 \mathrm{MPa}, 30^{\circ} \mathrm{C}$ & 0.872 \\
& $20 \mathrm{MPa}, 50^{\circ} \mathrm{C}$ & 0.632 \\
Non-buffered & $7 \mathrm{MPa}, 30^{\circ} \mathrm{C}$ & 0.274 \\
brine & $20 \mathrm{MPa}, 50^{\circ} \mathrm{C}$ & 0.179 \\
\hline
\end{tabular}




\section{B.4. Error Analysis}

Contact angle measurements can be challenging to reproduce because of the wide range of methodological hurdles associated with preparing and polishing homogenous surfaces, creating bubbles of the appropriate scale, equilibrium conditions and controlling variables, like temperature, pressure, and $\mathrm{pH}$ that can impact the magnitude of the measurement. In the experiments presented here, error bars were calculated to represent $90 \%$ confidence interval over at least eight replicate bubbles in two or more independent tests using different mineral specimens and brine in each replicate. The authors that led the experimental effort also had over one year of experience performing these measurements enabling them to build up the ability to perform the work in a reproducible manner. The LabView DAQ and controls on the system were also a factor in ensuring the reproducibility of the experiments by enabling precise control on temperature, pressure, and $\mathrm{pH}$ in the experimental high pressure cell.

\section{B.5. Effect of Bubble Size on Contact Angle}

The results reported here are based on the assumption that contact angle is independent of bubble size. We did observe subtle size effects in our work, consistent with published studies [Good and Koo, 1979]. To be consistent with the standard methods in measuring contact angle, we took pictures of bubbles with an average diameter of 2-3 mm. Even though this is 1-2 orders of magnitude larger than average pore throat size in GCS relevant porous media, scale is unlikely to impact these results for two reasons. First, the trends highlighted in this paper should hold across scales even if the magnitude of the contact angles changes slightly. Second, even though no phenomenological relationship between contact angle and bubble size has been proposed, 
primarily because of the case-specific role of surface heterogeneities and solution chemistry, the overall magnitude of the size effect is small relative to the other factors we report.

\section{B.6. Surface Characterization using SEM-EDS, AFM and Hirox Optical Mi- croscope}

Surface of phlogopite, silica, quartz and calcite were characterized using SEM-EDS, AFM and Hirox Optical Microscope. Phlogopite and silica surface are highly smooth with an average surface roughness in the range of 10 to $100 \mathrm{~nm}$ (Figure B.3). Quartz and calcite which were prepared using sand papers had significant higher surface roughness in the range of $25 \mu \mathrm{m}$.

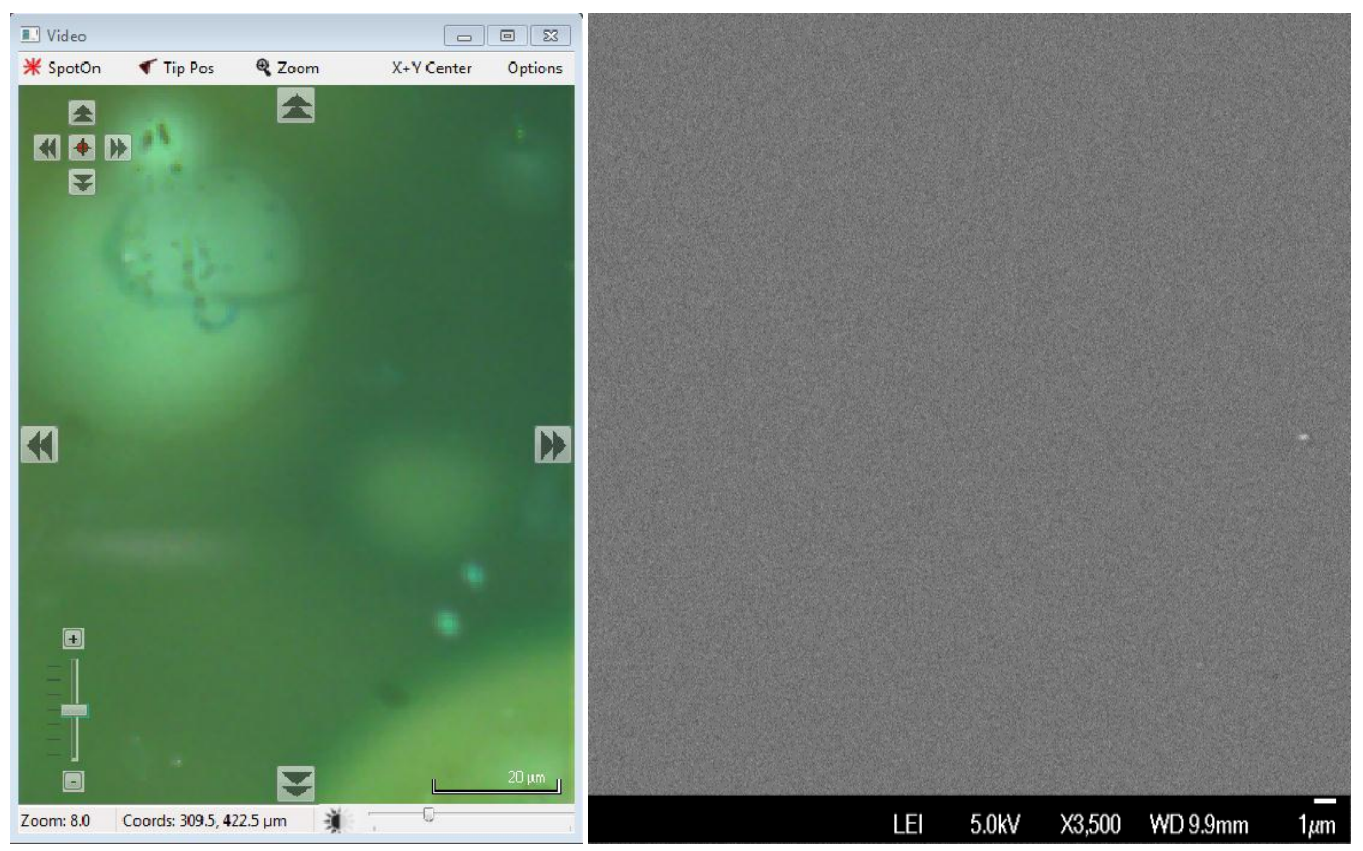

Figure B.3. SEM and AFM analyses showed atomatically smooth surface of phlogopite. (Left). AFM result (Right). SEM result 


\section{B.7. Effects of $\mathbf{p H}$ and ionic strength on Clay Species}

Clays generally exhibit complex interaction with the aqueous solutions they contact because of cation exchange, hydrolysis and dissolution and precipitation. Since hysteresis could also play an important role in long term stability of $\mathrm{CO}_{2}$ in the subsurface, hysteresis was characterized by measuring the advancing and receding contact angle on all the clay surfaces in a captive-bubble manner. This was done on phlogopite in particular since under acidic conditions it dissolves into the solution and can precipitate out of solution as kaolinite.

Here we conducted two sets of studies in which we do both $\mathrm{pH}$ titrations and ionic strength stepwise increases over a range of relevant ionic strength and $\mathrm{pH}$ conditions. The $\mathrm{pH}$ "sweep" tests were carried out from acidic to alkaline conditions at constant ionic strength. The salinity "sweep" tests were carried out by varying the ionic strength by three orders of magnitude, i.e. $0.01 \mathrm{M}, 0.1 \mathrm{M}$ and $1 \mathrm{M}$ at constant $\mathrm{pH}$. All experiments were conducted for clays with different surface structure (2:1 layered $=$ illite and phlogopite vs. 1:1 layered $=$ kaolinite $)$.

The results indicate that all the three clays show highly non-wetting behaviors under all conditions with phlogopite generally exhibiting slightly lower contact angle $\left(1 \sim 2^{\circ}\right)$. Salinity does not show discernible effects on contact angle. 


\section{B.8. Ion Selective Electrode (ISE) Analysis on Solution Chemistry}

Connection exists between the chemistry we're seeing on the mineral surface and the chemistry that is occurring in solution. Cation exchange is a very important phenomenon that links the solid-phase mineral surface with the aqueous phase. $\mathrm{K}^{+}$is the main cation species that exist in the crystal lattice of clay minerals. The cation exchange capacity (CEC) of $\mathrm{K}^{+}$for kaolinite, phlogopite, and illite clays was measured by using a $\mathrm{K}^{+}$selective electrode (Thermo Scientific, Inc.). The results indicated that $\mathrm{K}^{+}$activity measured in illite samples were significantly higher than phlogopite and kaolinite. This is not only due to the high CEC, but also because of the significant higher dissolution rate of illite into solution compared to the other two clays. 


\section{References}

1. Adamson, A. W. (1997), Physical Chemistry of Surfaces, 6th ed., Wiley, New York.

2. Al-Raoush, R. I., and C. S. Willson (2005), Extraction of physically realistic pore network properties from three-dimensional synchrotron X-ray microtomography images of unconsolidated porous media systems, Journal of Hydrology, 300 (1-4), 44-64, DOI:10.1016/j.jhydrol.2004.05.005.

3. Bachu, S. (2008), $\mathrm{CO}_{2}$ storage in geological media: Role, means, status and barriers to deployment, Progress in Energy and Combustion Science, 34(2), 254-273, DOI:10.1016/j.pecs.2007.10.001

4. Bachu, S., and J. J. Adams (2003), Sequestration of $\mathrm{CO}_{2}$ in geological media in response to climatechange: Capacity of deep saline aquifers to sequester $\mathrm{CO}_{2}$ in solution, Energy Conversion and Management, 44(20), 3151-3175, DOI: 10.1016/S0196-8904(03)001018.

5. Bachu, S., and D. Brant Bennion (2009), Dependence of $\mathrm{CO}_{2}$-brine interfacial tension on aquifer pressure, temperature and water salinity, Energy Procedia, 1(1), 3157-3164, DOI:10.1016/j.egypro.2009.02.098.

6. Bando, S.; F. Takemura; M. Nishio; E. Hihara; and M. Akai (2003), Solubility of $\mathrm{CO}_{2}$ in aqueous solutions of $\mathrm{NaCl}$ at (30 to 60$){ }^{\circ} \mathrm{C}$ and (10 to 20) $\mathrm{MPa}$, Journal of Chemical \& Engineering Data, 48(3), 576-579, DOI:10.1021/je0255832

7. Bando, S.; F. Takemura; M. Nishio; E. Hihara; and M. Akai (2004), Viscosity of aqueous $\mathrm{NaCl}$ solutions with dissolved $\mathrm{CO}_{2}$ at (30 to 60) ${ }^{\circ} \mathrm{C}$ and (10 to 20) $\mathrm{MPa}$, Journal of Chemical \& Engineering Data, 49(5), 1328-1332, DOI:10.1021/ je049940f. 
8. Barnes, H. A. (1997), Thixotropy-A review, Journal of Non-Newtonian Fluid Mechanics, 70(1-2), 1-33, DOI:10.1016/S0377-0257(97)00004-9.

9. Benson, S. M., and D. R. Cole (2008), $\mathrm{CO}_{2}$ sequestration in deep sedimentary formations, Elements, 4(5), 325-331, DOI:10.2113/gselements.4.5.325.

10. Bhattacharya, I. (2011), $\mathrm{CO}_{2}$ storage report, mountaineer commercial scale carbon capture and storage (CCS) project, Global CCS Institute.

11. Bikkina, P. K. (2011), Contact angle measurements of $\mathrm{CO}_{2}-$ water-quartz/calcite systems in the perspective of carbon sequestration, International Journal of Greenhouse Gas Control, 5(5), 1259-1271, DOI: 10.1016/ j.ijggc.2011.07.001.

12. Birkholzer, J. T., and Q. Zhou (2009), Basin-scale hydrogeologic impacts of $\mathrm{CO}_{2}$ storage: Capacity andregulatory implications, International Journal of Greenhouse Gas Control, 3(6), 745-756, DOI:10.1016/j.ijggc.2009.07.002.

13. Bleam, W. F. (1993), On Modeling Proton Affinity at the Oxide/Water Interface, Journal of Colloid and Interface Science, 159 (2), 312-318, DOI: 10.1006/jcis.1993.1328.

14. Boer, J. H. de (1936), ibid, 32 (10).

15. Brady, P. V. (1996), Physics and Chemistry of Mineral Surfaces, CRC Press: Boca Raton, New York, London, Tokyo.

16. Broseta, d.; N. Tonnet; and V. Shah (2012), Are rocks still water-wet in the presence of dense $\mathrm{CO}_{2}$ or $\quad \mathrm{H}_{2} \mathrm{~S}$ ?, Geofluids, $12 \quad$ (4), 280-294, $\quad$ DOI: $10.1111 / \mathrm{j} .1468-$ 8123.2012.00369.x.

17. Bruant, R. G. J.; M. A. Celia; A. J. Guswa; and C. A. Peters (2002), Safe storage of $\mathrm{CO}_{2}$ in deep saline aquifiers, Environmental Science \& Technology, 36(11), 240-245, DOI: 10.1021/es0223325. 
18. Buckley, J. (1996), Wetting alteration by brines and crude oil; from contact angles to cores, SPE Journal, 1 (3), 341-350, DOI: 10.2118/ 30765-PA.

19. Buckley, J. S.; K. Takamura; and N. R. Morrow (1989), Influence of Electrical Surface Charges on the Wetting Properties of Crude Oils, SPEFE August, 332-340, DOI: 10.2118/16964-PA.

20. Carre, A., and V. Lacarriere (2006), Study of surface charge properties of minerals and surface-modified substrates by wettability measurements, Contact Angle, Wettability, and Adhesion, 4, 1-14.

21. Chalbaud, C.; Lombard, J.-M.; Martin, F.; Robin, M.; H. Bertin; P. Egermann (2007)a, Two phase flow properties of brine- $\mathrm{CO}_{2}$ Systems in a carbonate core: influence of wettability on Pc and kr. In SPE/EAGE Reservoir Characterization and Simulation Conference, SPE: Abu Dhabi, UAE, 2007.

22. Chalbaud, C.; Robin, M.; Bekri, S.; Egermann, P. (2007)b, Wettability impact on $\mathrm{CO}_{2}$ storage in aquifers: visualisation and quantification using micromodel tests, pore network model and reservoir simulations, International Symposium of the Society of Core Analysts, Calgary, Canada, 2007; Calgary, Canada, 2007.

23. Chalbaud, C.; M. Robin; J. M. Lombard; F. Martin; P. Egermann; and H. Bertin (2009), Interfacial tension measurements and wettability evaluation for geological $\mathrm{CO}_{2}$ storage, Advances in Water Resources, 32 (1), 98-109, DOI: 10.1016/j.advwatres.2008.10.012.

24. Chen, C., and D. Zhang (2010), Pore-scale simulation of density-driven convection in fractured porous media during geological $\mathrm{CO}_{2}$ sequestration, Water Resources Research, 46(11), W11527, DOI:10.1029/2010WR009453. 
25. Chen, Q.; M. K. Gingras; and B. J. Balcom, (2003), A magnetic resonance study of pore filling processes during spontaneous imbibition in Berea sandstone, Journal of Chemical Physics, 119, 9609-9616, DOI:10.1063/1.1615757.

26. Chhabra, R. P., and J. F. Richardson (2008), Non-newtonian flow and applied rheology engineering applications, 2nd ed., Butterworth-Heinemann/Elsevier, Boston.

27. Chiquet, P.; D. Broseta; and S. Thibeau (2007)a, Wettability alteration of caprock minerals by carbon dioxide, Geofluids 7 (2), 112-122, DOI: $10.1111 / \mathrm{j} .1468$ 8123.2007.00168.x.

28. Chiquet, P., and D. Broseta (2005), Capillary alteration of shaly caprocks by carbon dioxide, In SPE Europec/EAGE Annual Conference, Society of Petroleum Engineers Madrid, Spain.

29. Chiquet, P.; J. L. Daridon; D. Broseta; and S. Thibeau (2007)b, $\mathrm{CO}_{2} /$ water interfacial tensions under pressure and temperature conditions of $\mathrm{CO}_{2}$ geological storage, Energy Conversion and Management, 48(3), 736-744, DOI:10.1016/ j.enconman.2006.09.011.

30. Cihan, A., and M. Y. Corapcioglu (2008), Effect of compressibility on the rise velocity of an air bubble in porous media, Water Resources Research, 44(4), W04409, DOI:10.1029/2006WR005415.

31. Cole, D. R.; Chialvo, A. A.; Rother, G.; Vlcek, L.; and Cummings, P.T. (2010). Supercritical fluid behavior at nanoscale interfaces: implications for $\mathrm{CO}_{2}$ sequestration in geologic formations. Philosophical Magazine., 90(17-18), 2339-2363.

32. Corapcioglu, M. Y.; A. Cihan; and M. Drazenovic (2004), Rise velocity of an air bubble in porous media: Theoretical studies, Water Resources Research, 40(4), W04214, DOI:10.1029/2003WR002618. 
33. Crandell, L. E.; B. R. Ellis; and C. A. Peters (2010), Dissolution potential of $\mathrm{SO}_{2}$ coinjected with $\mathrm{CO}_{2}$ in geologic sequestration, Environmental Science \& Technology, 44(1), 349-355, DOI:10.1021/es902612m.

34. Duc, M.; F. Gaboriaud; and F. Thomas (2005), Sensitivity of the acid-base properties of clays to the methods of preparation and measurement: 1. Literature review, Journal of Colloid and Interface Science, 289 (1), 139-147, DOI: 10.1016/j.jcis.2005.03.060.

35. DePaolo, D. J., and Orr, F. M. (2008). Geoscience research for our energy future. Physics Today, 61(8), 46-51, DOI: 10.1063/1.2970212

36. Djéridi, H.; J. F. Favé; J. Y. Billard; and D. H. Fruman (1999), Bubble capture and migration in Couette-Taylor flow, Experiments in Fluids, 26(3), 233-239, DOI:10.1007/s003480050284.

37. Dooley, J.; R. Dahowski; C. Davidson; M. Wise; N. Gupta; S. Kim; and E. L. Malone (2006), Carbon dioxide capture and geologic storage - A core element of a global energy technology strategy to address climate change, in Joint Global Change Research Institute (JGCRI), pp. 37, Battelle Memorial Institute, College Park Maryland.

38. Duan, Z.; R. Sun; C. Zhu; and I. Chou (2006), An improved model for the calculation of $\mathrm{CO}_{2}$ solubility in aqueous solutionscontaining $\mathrm{Na}^{+}, \mathrm{K}^{+}, \mathrm{Ca}^{2+}, \mathrm{Mg}^{2+}, \mathrm{Cl}^{-}$, and $\mathrm{SO}_{4}{ }^{2-}, \mathrm{Ma}-$ rine Chemistry, 98(2-4), 131-139, DOI:10.1016/j.marchem.2005.09.001.

39. Dutton, S. P.; and Willis, B. (1998), Comparison of Outcrop and Subsurface Sandstone Permeability Distribution, Lower Cretaceous Fall River Formation, South Dakota and Wyoming, Journal of Sedimentary Research, 68 (5), 890-900, DOI: 10.1306/D42688A62B26-11D7-8648000102C1865D 
40. Eccles, J. K.; L. Pratson; R. G. Newell; and R. B. Jackson (2009), Physical and economic potential of geological $\mathrm{CO}_{2}$ storage in saline aquifers, Environmental Science \& Technology, 43 (6), 1962-1969, DOI:10.1021/es801572e.

41. Ellis, B.; Peters, C.; Fitts, J.; Bromhal, G.; McIntyre, D.; Warzinski, R.; and Eilis, R. (2011). Deterioration of a fractured carbonate caprock exposed to $\mathrm{CO}_{2}$-acidified brine flow. Greenhouse Gases: Science and Technology, 1(3), 248-260, DOI: 10.1002/ghg.25

42. Ergun, S. (1952), Mass-transfer rate in packed columns, Chemical Engineering Progress, $48,89-94$.

43. Espinoza, D. N.; and J. C. Santamarina (2010), Water- $\mathrm{CO}_{2}$-mineral systems: Interfacial tension, contact angle, and diffusion - implications to $\mathrm{CO}_{2}$ geological storage, Water Resources Research, 46 (7), W07537, DOI:10.1029/2009WR008634.

44. Garcia, J. E. (2001), Density of aqueous solutions of $\mathrm{CO}_{2}$, Lawrence Berkeley National Lab, CA.

45. Gasda, S. E.; J. M. Nordbotten; and M. A. Celia (2011), Vertically averaged approaches for $\mathrm{CO}_{2}$ migration with solubility trapping, Water Resources Research, 47(5), W05528, DOI:10.1029/2010WR009075.

46. Giammar, D. E.; R. G. Bruant Jr; and C. A. Peters (2005), Forsterite dissolution and magnesite precipitation at conditions relevant for deep saline aquifer storage and sequestration of carbon dioxide, Chemical Geology, 217 (3-4), 257-276, DOI: 10.1016/j.chemgeo.2004.12.013.

47. Glover, P.W.J.; and E. Walker (2011), Grain-size to effective pore-size transformation derived from electrokinetic theory, Geophysics, 76(4), 17-29 
48. Good, R. J.; Koo, M. N. (1979), The effect of drop size on contact angle. Journal of Colloid and Interface Science 71 (2), 283-292.

49. Hamaker, H. C. (1937), Physica, 4 (1058).

50. Hebach, A.; A. Oberhof; N. Dahmen; A. Kögel; H. Ederer; and E. Dinjus (2002), Interfacial tension at elevated pressures measurements and correlations in the water + carbon dioxide system, Journal of Chemical \& Engineering Data, 47(6), 1540-1546, DOI: $10.1021 /$ je025569p.

51. IPCC. (2005), Carbon dioxide capture and storage. Intergovernmental Panel on Climate Change, Geneva, Switzerland.

52. Israelachvili, J. N. (2011), Intermolecular and Surface Forces. 3rd ed.; Academic Press: Burlington, MA.

53. Israelachvili, J. N.; Tabor, D. (1973). The shear properties of molecular films. Wear, 24(3), 386-390.

54. Ji, Y.; Ji, X.; Feng, X.; Liu, C.; Lu, L.; Lu, X.; and Chin, J. (2007). Progress in the study on the phase equilibria of the $\mathrm{CO}_{2}-\mathrm{H}_{2} \mathrm{O}$ and $\mathrm{CO}_{2}-\mathrm{H}_{2} \mathrm{O}-\mathrm{NaCl}$ systems. Chinese Journal of Chemical Engineering, 15(3), 439-448, DOI: 10.1016/S1004-9541(07)60105-0.

55. Joh, S. W.; S. H. Lee; and J. R. Youn (2010), Rheological behavior of polydispersed bubble suspensions in shear flows, Polymer Engineering \& Science, 50(1), 128-137, DOI:10.1002/pen.21517.

56. Juanes, R.; E. J. Spiteri; F. M. J. Orr; and M. J. Blunt (2006), Impact of relative permeability hysteresis on geological $\mathrm{CO}_{2}$ storage. Water Resources Research, 42(12), W12418, DOI:10.1029/2005WR004806. 
57. Kendall, K. (1994), Adhesion: molecules and mechanics, Science, 263, 1720-1725, DOI: 10.1126/science.263.5154.1720.

58. Kerisit, S.; Cooke, D. J.; Spagnoli, D.; and Parker, S. C. (2005). Molecular dynamics simulations of the interactions between water and inorganic solids. Journal of Materials Chemistry, 15(14), 1454-1462. DOI: 10.1039/B415633C.

59. Kijevčanin, M. L.; V. Z. Kostić; I. R. Radović; B. D. Đorđević; and S. P. Šerbanović (2008), Viscosity of binary non-electrolyte liquid mixtures: Prediction and correlation, Chemical Industry and Chemical Engineering Quarterly, 14(4), 223-226, DOI:10.2298/CICEQ0804223K.

60. Kim, Y.; Wan, J.; Kneafsey, T. J.; and Tokunaga, T. K.(2012). Dewetting of silica surfaces upon reactions with supercritical $\mathrm{CO}_{2}$ and brine: porescale studies in micromodels. Environmental Science and Technology, 46 (7), 4228-4235. DOI: 10.1021/es204096w.

61. Klempner, D.; and K. C. Frisch (1991), Handbook of polymeric foams and foam technology, Hanser, New York.

62. Kloek, W.; T. van Vliet; and M. Meinders (2001), Effect of bulk and interfacial rheological properties on bubble dissolution, Journal of Colloid and Interface Science, 237(2), 158-166, DOI:10.1006/jcis.2001.7454.

63. Kneafsey, T.; and K. Pruess (2010), Laboratory flow experiments for visualizing carbon dioxide-induced, density-driven brine convection, Transport in Porous Media, 82(1), 123-139, DOI:10.1007/s11242-009-9482-2.

64. Kumagai, A.; and C. Yokoyama (1998), Falling capillary tube viscometer suitable for liquids at high pressure, Review of Scientific Instruments, 69, 1441-1445, DOI:10.1063/1.1148778. 
65. Kumagai, A.; and C. Yokoyama (1999), Viscosities of aqueous $\mathrm{NaCl}$ solutions containing $\mathrm{CO}_{2}$ at high pressures, Journal of Chemical \& Engineering Data, 44(2), 227-229, DOI:10.1021/je980178p.

66. Kumar, A.; Noh, M.; Pope, G.A.; Sepehrnoori, K.; and Bryant, S. (2005). Reservoir simulation of $\mathrm{CO}_{2}$ storage in deep saline aquifers. SPE Journal, 10(3), 336-348.

67. Kumar, P. P.; A. G. Kalinichev; and R. J. Kirkpatrick (2008), Hydrogen-bonding structure and dynamics of aqueous carbonate species from Car-Parrinello molecular dynamics simulations, The Journal of Physical Chemistry B, 113(3), 794-802, DOI:10.1021/jp809069g.

68. Lackner, K. S. (2003), A Guide to $\mathrm{CO}_{2}$ Sequestration, Science, 300(5626), 1677-1678, DOI:10.1126/science.1079033.

69. Lake, L. W. (1989). Enhance oil recovery Old Tappan, NJ; Prentice Hall Inc.

70. Laliberte, M. (2007), Model for calculating the viscosity of aqueous solutions, Journal of Chemical \& Engineering Data, 52(2), 321-335, DOI:10.1021/je0604075.

71. Levine, J. S.; J. M. Matter; D. S. Goldberg; K. S. Lackner; M. G. Supp; and T. S. Ramakrishnan (2011), Two phase brine- $\mathrm{CO}_{2}$ flow experiments in synthetic and natural media. Energy Procedia 4 (0), 4347-4353, DOI: 10.1016/j.egypro.2011.02.386.

72. Li, L.; C. A. Peters; and M. A. Celia (2006), Upscaling geochemical reaction rates using pore-scale network modeling, Advances in Water Resources, 29(9), 1351-1370, DOI:10.1016/j.advwatres.2005.10.011.

73. Lívanský, K. (1982), Effect of temperature and pH on absorption of carbon dioxide by a free level of mixed solutions of some buffers, Folia Microbiologica 27 (1), 55-59, DOI: 10.1007/bf02883839. 
74. Live Science. (2009). Rocks found that could store greenhouse gas. Retrieved 1/18, 2013, from http://www.livescience.com/3364-rocks-store-greenhouse-gas.html

75. Llewellin, E. W.; and M. Manga (2005), Bubble suspension rheology and implications for conduit flow, Journal of Volcanology and Geothermal Research, 143(1-3), 205-217, DOI:10.1016/j.jvolgeores.2004.09.018.

76. Llewellin, E. W.; H. M. Mader; and S. D. R. Wilson (2002a), The rheology of a bubbly liquid, Proceedings of the Royal Society of London. Series A: Mathematical, Physical and Engineering Sciences, 458. (2020) pp. 987-1016,

77. Llewellin, E. W.; H. M. Mader; and S. D. R. Wilson (2002b), The constitutive equation and flow dynamics of bubbly magmas, Geophysical Research Letters, 29(24), 2170, DOI:10.1029/2002GL015697.

78. Manga, M.; and M. Loewenberg (2001), Viscosity of magmas containing highly deformable bubbles, Journal of Volcanology and Geothermal Research, 105(1-2), 19-24, DOI:10.1016/S0377-0273(00)00239-0.

79. Mansoori, S. A., Iglauer, S., Pentland, C. H., Bijeljic, B., and Blunt, M.J. (2009). Measurements of non-wetting phase trapping applied to carbon dioxide storage. Energy Procedia, 1(1), 3173-3180, DOI: 10.1016/j.ijggc.2009.09.013

80. McCool, B.; Tripp, C. L. (2005), Inaccessible hydroxyl groups on silica are accessible to supercritical $\mathrm{CO}_{2}$. The Journal of Physical Chemistry, B, 109, 8914-8919, DOI: 10.1021/jp050192q.

81. McGinnis, D. F.; J. Greinert; Y. Artemov; S. E. Beaubien; and A. Wuest (2006), Fate of rising methane bubbles in stratified waters: How much methane reaches the atmosphere? Journal of Geophysical Research - Oceans, 111, C09007, DOI: 10.1029/2005JC003183 
82. McKaveney, J. P.; and D. Stivers (1978), Capacity of some California alkaline brines for sulfur dioxide, American Chemical Society - Energy and Fuel Division, Anaheim, CA; Occidental Research Corporation: Anaheim, CA.

83. Mills, J.; M. Riazi; and M. Sohrabi (2011), Wettability of common rock forming minreals in a $\mathrm{CO}_{2}$-brine system at reservoir conditions, The International Symposium of the Society of Core Analysts, Austin, Texas, USA.

84. MIT. (2007). The future of coal. Cambridge MA: Massachusetts Institute of Technology.

85. Moffat, J. R.; Sefiane, K.; Shanahan, M. E. R. (2009), Effect of $\mathrm{TiO}_{2}$ nanoparticles on contact line stick-slip behavior of volatile drops, The Journal of Physical Chemistry B 113, (26), 8860-8866, DOI: 10.1021/jp902062z

86. Morrow, N. R.; G.-q. Tang; M. Valat; and X. Xie (1998), Prospects of improved oil recovery related to wettability and brine composition, Journal of Petroleum Science and Engineering, 20 (3-4), 267-276, DOI: 10.1016/s0920-4105(98)00030-8.

87. Murai, Y.; and H. Oiwa (2008), Increase of effective viscosity in bubbly liquids from transient bubble deformation, Fluid Dynamics Research, 40(7-8), 565-575, DOI:10.1016/j.fluiddyn.2007.12.009.

88. Nakken, T.; M. Tande; and A. Elgsaeter (2001), Measurements of polymer induced drag reduction and polymer scission in Taylor flow using standard double-gap sample holders with axial symmetry, Journal of Non-Newtonian Fluid Mechanics, 97(1), 1-12, DOI:10.1016/S0377-0257(00)00195-6.

89. National Research Council. (2010). Limiting the magnitude of future climate change, America's climate choices. Washington, DC: The National Academies Press. 
90. Nordbotten, J. M.; M. A. Celia; S. Bachu; and H. K. Dahle (2004), Semianalytical solution for $\mathrm{CO}_{2}$ leakage through an abandoned well. Environmental Science \& Technology, 39(2), 602-611, DOI:10.1021/es035338i.

91. Oldenburg, C.M.; and J.L. Lewicki (2005), Leakage and seepage of $\mathrm{CO}_{2}$ from geologic carbon sequestration sites: $\mathrm{CO}_{2}$ migration into surface water, Lawrence Berkeley National Laboratory Report, LBNL-57768, June 2005.

92. Oldenburg, C. M. (2007)a. Joule-thomson cooling due to $\mathrm{CO}_{2}$ injection into natural gas reservoirs. Energy Conversion and Management, 48(6), 1808-1815, DOI: 10.1016/j.enconman.2007.01.010.

93. Oldenburg, C. M. (2007)b, Migration mechanisms and potential impacts of $\mathrm{CO}_{2}$ leakage and seepage, in Carbon Capture and Storage, edited by E. Wilson and D. Gerard, Blackwell Publ., Ames, IA, 127-146.

94. Oldenburg, C.M.; and J. Lewicki (2006), On leakage and seepage of $\mathrm{CO}_{2}$ from geologic storage sites into surface water, Environmental Geology, 50(5), 691-705, DOI:10.1007/s00254-006-0242-0.

95. Oldenburg, C.; J. Lewicki; L. Pan; L. Dobeck; and L. Spangler (2010), Origin of the patchy emission pattern at the ZERT $\mathrm{CO}_{2}$ release test, Environmental Earth Sciences, 60(2), 241-250, DOI:10.1007/s12665-009-0442-5.

96. Padhy, G. S.; C. Lemaire; E. S. Amirtharaj; and M. A. Ioannidis (2007), Pore size distribution in multiscale porous media as revealed by DDIF-NMR, mercury porosimetry and statistical image analysis, Colloids and Surfaces A: Physicochemical and Engineering Aspects, 300 (1-2), 222-234, DOI:10.1016/j.colsurfa.2006.12.039.

97. Papirer, E. (2000), Adsorption on Silica Surfaces, CRC Press: Boca Raton, FL, 2000. 
98. Patzek, T. W.; D. B. Silin; S. M. Benson; and G. I. Barenblatt (2003), On vertical diffusion of gases in a horizontal reservoir, Transport in Porous Media, 51(2), 141-156, DOI:10.1023/A:1021957416536.

99. Peters, C. A. (2009), Accessibilities of reactive minerals in consolidated sedimentary rock: An imaging study of three sandstones, Chemical Geology, 265(1-2), 198-208, DOI:10.1016/j.chemgeo.2008.11.014.

100. Poletto, M.; and D. D. Joseph (1995), The effect of density and viscosity of a suspension, Journal of Rheology, 39(2), 323-343.

101. Pollak, M. F.; and E. J. Wilson (2009), Regulating geologic sequestration in the united states: Early rules take divergent approaches, Environmental Science \& Technology, 43(9), 3035-3041, DOI:10.1021/es803094f.

102. Portier, S., and Rochelle, C. (2005). Modelling $\mathrm{CO}_{2}$ solubility in pure water and NaCltype waters from 0 to $300{ }^{\circ} \mathrm{C}$ and from 1 to 300 bar: Application to the utsira formation at sleipner. Chemical Geology, 217(3-4), 187-199.

103. Pruess, K. (2004), The TOUGH Codes-A family of simulation tools for multiphase flow and transport processes in permeable media. Vadose Zone Journal, 3(3), 738-746, DOI: $10.2113 / 3.3 .738$.

104.Pruess, K. (2005), $\mathrm{ECO}_{2} \mathrm{~N}:$ A TOUGH2 fluid property module for mixtures of. water, $\mathrm{NaCl}$, and $\mathrm{CO}_{2}$. No. LBNL-57952. University of California, Berkeley, CA 94720: Earth Sciences Division, Lawrence Berkeley National Laboratory.

105.Pruess, K. (2006), On leakage from geologic storage reservoirs of $\mathrm{CO}_{2} \cdot \mathrm{CO}_{2} \mathrm{SC}$ Symposium 2006, Lawrence Berkeley National Laboratory, Berkeley, California. 
106. Pruess, K. (2008a), On $\mathrm{CO}_{2}$ fluid flow and heat transfer behavior in the subsurface, following leakage from a geologic storage reservoir, Environmental Geology, 54(8), 16771686, DOI:10.1007/s00254-007-0945-X.

107. Pruess, K. (2008b), Leakage of $\mathrm{CO}_{2}$ from geologic storage: Role of secondary accumulation at shallow depth, International Journal of Greenhouse Gas Control, 2(1), 37-46, DOI:10.1016/S1750-5836(07)00095-3.

108. Raveendran, P.; Y. Ikushima; and S. L. Wallen (2005), Polar attributes of supercritical carbon dioxide, Accounts of Chemical Research, 38 (6), 478-485, DOI: 10.1021/ar040082m.

109. Riaz, A.; and H. A. Tchelepi (2008), Dynamics of vertical displacement in porous media associated with $\mathrm{CO}_{2}$ sequestration, SPE Journal, 13(3), 305-313, DOI:10.2118/103169PA.

110. Roosevelt, S. E.; and M. Y. Corapcioglu (1998), Air bubble migration in a granular porous medium: Experimental studies, Water Resources Research, 34(5), 1131-1142, DOI:10.1029/98WR00371.

111. Rumpf, B.; and Maurer, G. (1993). An experimental and theoretical investigation on the solubility of carbon dioxide in aqueous solutions of strong electrolytes. Berichte Der Bunsengesellschaft fr Physikalische Chemie, $97(1), \quad 85-97, \quad$ DOI: 10.1002/bbpc. 19930970116

112. Sakurovs, R.; and S. Lavrencic (2011), Contact angles in $\mathrm{CO}_{2}$-water-coal systems at elevated pressures, International Journal of Coal Geology, 87 (1), 26-32, DOI: 10.1016/j.coal.2011.04.005. 
113.Sang-Yoon, K.; S. S. Ashok; T. Heng-Kwong; and L. K. Donald (1997), Rheology of dense bubble suspensions, Physics of Fluids, 9(6), 1540-1561, DOI: 10.1063/1.869481.

114. Schroth, B. K.; and G. Sposito (1997), Surface charge properties of kaolinite, Clays and Clay Minerals 45 (1), 85-91, DOI: 10.1557/PROC-432-87.

115. Shao, H.; J. R. Ray; and Y.-S. Jun (2011), Effects of salinity and the extent of water on supercritical $\mathrm{CO}_{2}$-induced phlogopite dissolution and secondary mineral formation. Environmental Science and Technology, 45 (4), 1737-1743, DOI: 10.1021/es1034975.

116. Silin, D.; T. W. Patzek; and S. M. Benson (2009), A one-dimensional model of vertical gas plume migration througha heterogeneous porous medium, International Journal of Greenhouse Gas Control, 3(3), 300-310, DOI:10.1016/j.ijggc.2008.09.003.

117. Sochi, T. (2010), Flow of non-newtonian fluids in porous media, Journal of Polymer Science Part B: Polymer Physics, 48(23), 2437-2767, DOI:10.1002/polb.22144.

118. Soli, A. L.; and R. H. Byrne (2002), $\mathrm{CO}_{2}$ system hydration and dehydration kinetics and the equilibrium $\mathrm{CO}_{2} / \mathrm{H}_{2} \mathrm{CO}_{3}$ ratio in aqueous $\mathrm{NaCl}$ solution, Marine Chemistry, 78(2-3), 65-73, DOI:10.1016/S0304-4203(02)00010-5.

119. Somasundaran, P.; and G. E. Agar (1967), The zero point of charge of calcite, Journal of Colloid and Interface Science, 24, 433-440, DOI: 10.1016/0021- 9797(67)90241-X.

120. Song, Y. Q. (2001), Pore sizes and pore connectivity in rocks using the effect of internal field, Magnetic Resonance Imaging, 19(3-4), 417-421, DOI:10.1016/S0730$725 \mathrm{X}(01) 00259-4$.

121. Soubiran, J.; and J. D. Sherwood (2000), Bubble motion in a potential flow within a venture, International Journal of Multiphase Flow, 26(11), 1771-1796, DOI:10.1016/S03019322(99)00113-5. 
122.Span, R.; E. W. Lemmon; R. T. Jacobsen; W. Wagner; and A. Yokozeki (2000), A reference equation of state for the thermodynamic properties of nitrogen for temperatures from 63.151 to $1000 \mathrm{~K}$ and pressures to $2200 \mathrm{MPa}$, Journal of Physical and Chemical Reference Data, 29(6), 1361-1433, DOI:10.1063/ 1.1349047.

123.Stumn, W. (1992), Chemistry of the Solid-Water Interface: Processes at the MineralWater and Particle-Water Interface in Natural Systems, John Wiley \& Sons, New York.

124. Sverjensky, D. A. (1994), Zero-point-of-charge prediction from crystal chemistry and solvation theory, Geochimica Et Cosmochimica Acta, 58 (14), 3123-3129, DOI: 10.1016/0016-7037(94)90184-8.

125. Tabor, D. F. R. S.; Winterton, R. H. S. (1969). The direct measurement of normal and retarded van der Waals forces. Proceedings of the Royal Society of London. A. Mathematical and Physical Sciences, 312(1511), 435-450. DOI:10.1098/rspa.1969.0169.

126. Taku Ide, S.; Jessen, K.; and Orr Jr., F.M. (2007), Storage of $\mathrm{CO}_{2}$ in saline aquifers: Effects of gravity, viscous, and capillary forces on amount and timing of trapping. International Journal of Greenhouse Gas Control, 1(4), 481-491, DOI: 10.1016/S17505836(07)00091-6.

127. Tewes, F.; and F. Boury (2005), Formation and rheological properties of the supercritical $\mathrm{CO}_{2}$ - water pure interface, The Journal of Physical Chemistry B, 109, 3900-3997, DOI:10.1021/jp046019w.

128. Thompson, M. J.; J. R. A. Pearson; and M. R. Mackley (2001), The effect of droplet extension on the rheology of emulsions of water in alkyd resin, Journal of Rheology, 45(6), 1341-1358, DOI:10.1122/1.1410371. 
129. Tokunaga, T. (2012), DLVO-based estimates of adsorbed water film thicknesses in geologic $\mathrm{CO}_{2}$ reservoirs, Langmuir, 28 (21), 8001-8009, DOI: 10.1021/la2044587.

130. Tomida, D.; A. Kumagai; and C. Yokoyama (2007), Viscosity measurements and correlation of the squalane $+\mathrm{CO}_{2}$ mixture, International Journal of Thermophysics, 28(1), 133-145, DOI: 10.1007/s10765-007-0149-3.

131. Tonnet N; Broseta D; Mouronval G (2010) Evaluation of the petrophysical properties of a carbonate-rich caprock for $\mathrm{CO}_{2}$ geological storage purposes. Paper SPE 131525, EUROPECEAGE Annual Conference and Exhibition held in Barcelona, Spain, 14-17 June.

132. Tripp, C. P.; and J. R. Combes (1998), Chemical modification of metal oxide surfaces in supercritical $\mathrm{CO}_{2}$ : The interaction of supercritical $\mathrm{CO}_{2}$ with the adsorbed water layer and the surface hydroxyl groups of a silica surface, Langmuir, 14 (26), 7348-7352, DOI: 10.1021/la9805701.

133. Vishnyakov, A.; Shen, Y.; Tornassone, M.S. (2008), Interactions of silica nanoparticles in supercritical carbon dioxide. The Journal of Chemical Physics, 129, 174704, DOI: 10.1063/1.2994714.

134. Vorholz, J.; Harismiadis, V. I.; Panagiotopoulos, A.; Rumpf, B.; and Maurer, G. (2004), Molecular simulation of the solubility of carbon dioxide inaqueous solutions of sodium chloride. Fluid Phase Equilibria, 226, 237-250, DOI: 10.1016/j.fluid.2004.09.009.

135. Wallis, G. B. (1969), One-Dimensional Two-Phase Flow, 408 pp., McGraw-Hill, New York.

136. Wang, S.; and A. Clarens (2012), The effects of $\mathrm{CO}_{2}$-brine rheology on leakage processes in geologic carbon sequestration, Water Resources Research, 48, W08518, PP.14, DOI:10.1029/2011WR011220. 
137. Wang, S.; Edwards, I.; and A. Clarens (2013), Wettability phenomena at the $\mathrm{CO}_{2}$-brinemineral interface: Implications for geologic carbon sequestration, Environmental Science and Technology, 47 (1), 234-241, DOI: 10.1021/es301297z.

138. Wang, Y.; Bryan, C.; Dewers, T.; Heath, J.; and Jove-Colon, C. (2013), Ganglion dynamics and its implications to geologic carbon dioxide storage, Environmental Science and Technology, 47 (1), pp 219-226, DOI: 10.1021/es301208k

139. Widjajakusuma, J.; B. Biswal; and R. Hilfer (1999), Quantitative prediction of effective material properties of heterogeneous media, Computational Materials Science, 16(1-4), 70-75, DOI:10.1016/S0927-0256(99)00047-6.

140.Wollenweber, J.; S. Alles; A. Busch; B. M. Krooss; H. Stanjek; and R. Littke (2010), Experimental investigation of the $\mathrm{CO}_{2}$ sealing efficiency of caprocks, International Journal of Greenhouse Gas Control, 4(2), 231-241, DOI:10.1016/ j.ijggc.2010.01.003.

141. Yang, D.; Y. Gu; and P. Tontiwachwuthikul (2008), Wettability Determination of the Reservoir Brine-Reservoir Rock System with Dissolution of $\mathrm{CO}_{2}$ at High Pressures and Elevated Temperatures, Energy \& Fuels 22 (1), 504-509, DOI: 10.1021/ef700383x.

142. Yang, C.; and Y. Gu (2006). Accelerated mass transfer of $\mathrm{CO}_{2}$ in reservoir brine due to density-driven natural convection at high pressures and elevated temperatures, Industrial \& Engineering Chemistry Research, 45(8), 2430-2436, DOI:10.1021/ ie050497r.

143.Zafarani-Moattar, M. T.; and R. Majdan-Cegincara (2009), Viscosity modeling and prediction of aqueous mixed electrolyte solutions, Industrial \& Engineering Chemistry Research, 48(12), 5833-5844, DOI:10.1021/ie801933u.

144.Zhang, C.; K. Dehoff, N. Hess; M. Oostrom; T. W. Wietsma; A. J. Valocchi; B. W. Fouke; and C. J. Werth (2010), Pore-scale study of transverse mixing induced $\mathrm{CaCO}_{3}$ 
precipitation and permeability reduction in a model subsurface sedimentary system, Environmental Science \& Technology, 44 (20), 7833-7838, DOI: 10.1021/es1019788.

145.Zhang, J.; and L. S. Fan (2003), On the rise velocity of an interactive bubble in liquids, Chemical Engineering Journal, 92(1-3), 169-176, DOI:10.1016/S1385-8947(02) 001894.

146.Zhang, Y.; C. M. Oldenburg; S. Finsterle; P. Jordan; and K. Zhang (2009), Probability estimation of $\mathrm{CO}_{2}$ leakage through faults at geologic carbon sequestration sites, Energy Procedia, 1(1), 41-46, DOI:10.1016/j. egypro.2009.01.008. 